DEPARTMENT OF TRANSPORTATION COAST GUARD

\title{
OCEANOGRAPHY of the NEW YORK BIGHT
}

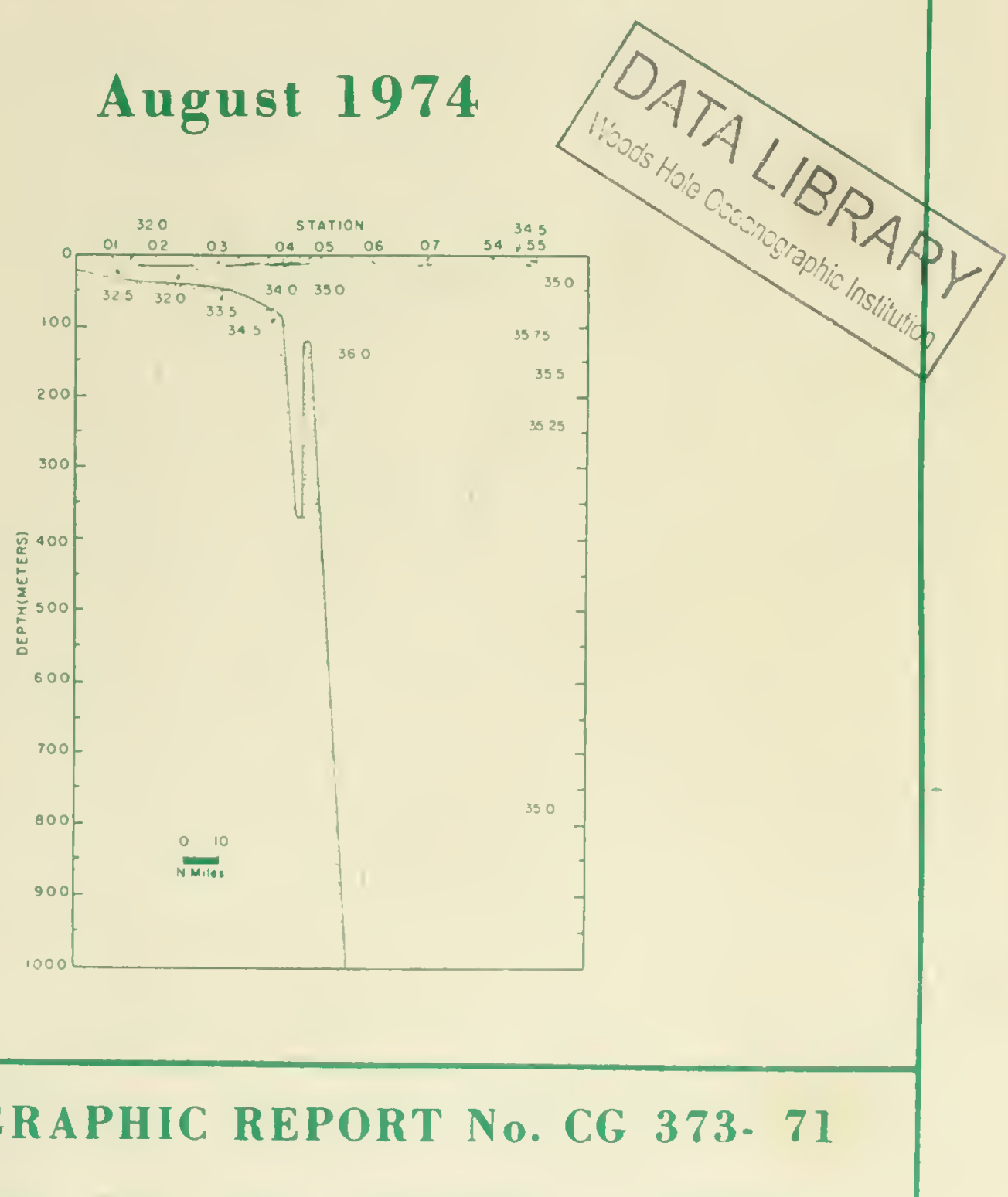

no. 71 



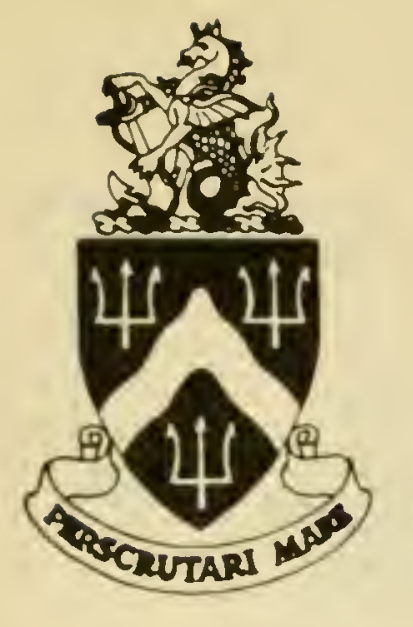

\section{OCEANOGRAPHIC REPORT \\ No. CG 373-71}

\section{OCEANOGRAPHY of the NEW YORK BIGHT August 1974}

Charles W. Morgan

Joseph M. Bishop

Frank F. Mulher

June 1976

United States Coast Guard

Oceanographic Unit

Washington, D.C. 


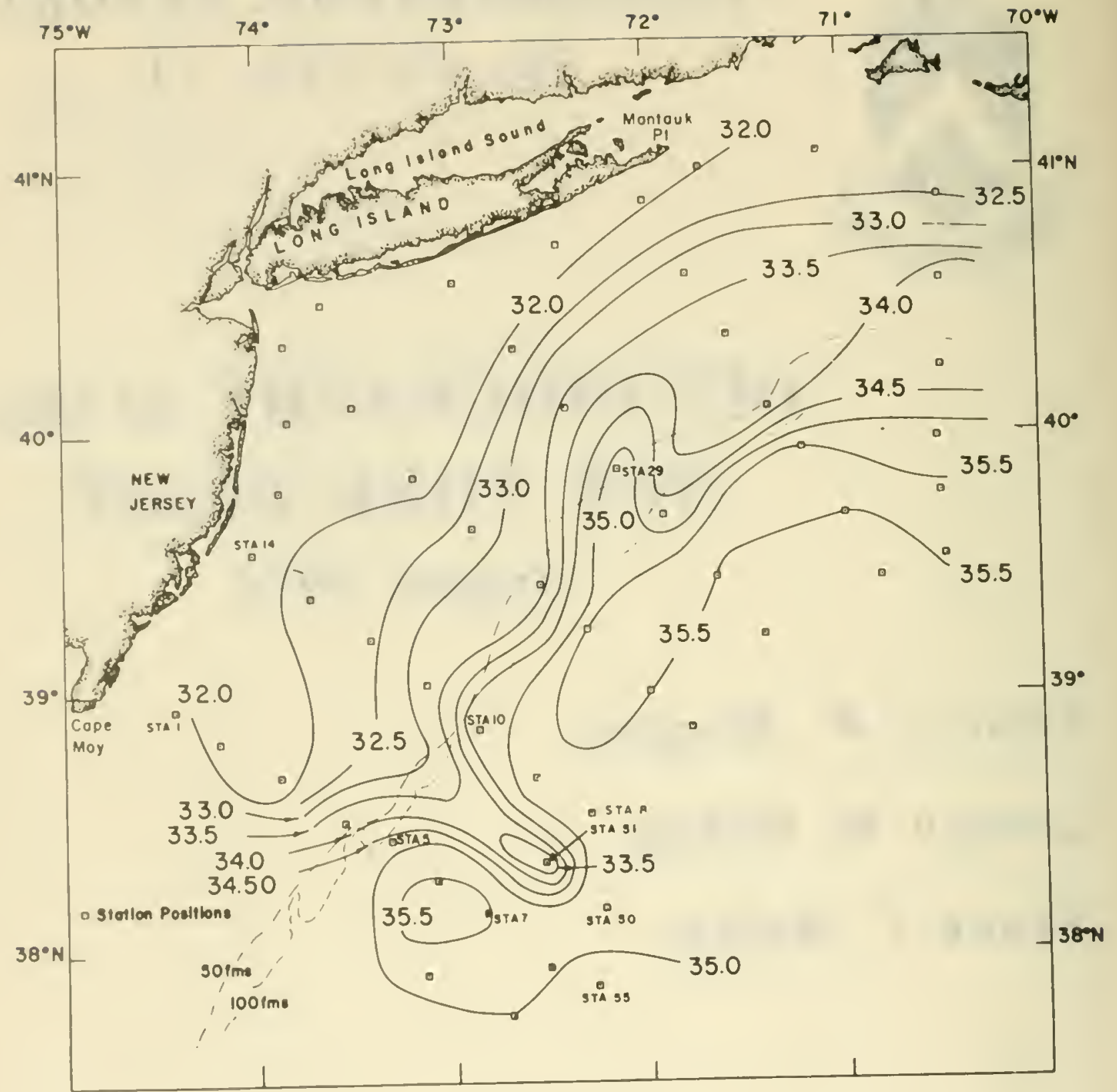

5) metrer salinity field 


\section{ABSTRACT}

The physical oceanography of the shelf and slope waters of the New York Bight (Block Island to Cape May) in August of 1974 is described. 'Temperature, salinity, and density data, presented in surface contours and section profiles, showed the shelf/slope front, a cold core on the shelf, and a salinity core on the slope. Geostrophic currents in the slope water were inferred from the density structure, and showed two anticyelonic eddies with maximum geostrophic relocities of approximately $40 \mathrm{~cm} \mathrm{~s} \mathrm{~s}^{-1}$. Temperature and salinity profiles indicated shelf/slope mixing related to the eddies.

Editor's Note: Reference to a product or comment with respect to it in this publication does not indicate, or permit any person to held out hy republication in whole or in part or otherwise, that the product has been endorsed, anthorized, ur aproved by" the Coast Guard. 



\section{TABLE OF CONTENTS}

Title Page _...

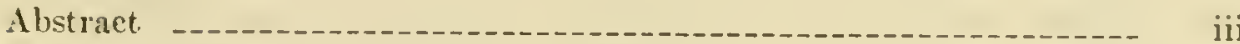

Table of Contents _.

List of Illustrations -

List of Tables

Introduction -..-1-

Procedures - -

Oceanographic Sampling -

Quality Control _. 3

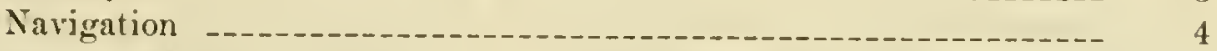

Current Meters -

Data Presentation _..-_- 5

Data Listing -

Surface Contours _...-

Mean Vertically Areraged Sigma-t, $\sigma_{\mathrm{t}} \ldots \ldots \ldots \ldots \ldots \ldots-\ldots$

Dynamic Height Contours _._._._._. 5

Vertical Section Contours _...

Results -.-.-

Cold Core - -

High Salinity Core of Slope Water _..._- 7

Temperature-Salinity Correlations

Circulation -...

An Anticyclonic Eddy in the Slope Water _._._._. 8

References -._-_._- 10

Illustrations --

Appendix A-Oceanographic Data _._- 40

\section{LIST OF ILLUSTRATIONS}

Figure

1. Station and section locations, August $1974 \ldots \ldots \ldots$

2. Data processing flow diagram _._. 12

3. Sea surface temperature distribution, $8-20$ August $1974\left({ }^{\circ} \mathrm{C}\right) \ldots$

4. Sea surface salinity distribution, 8-20 August $1974(\%$.

5. Sea surface temperatures from August 1974 ART flight; track of eddy $\left({ }^{\circ} \mathrm{C}\right)$

6a. Mean vertically averaged sigma-t, Angust 1974. (Arrows show current computed from Bishop, 1975)

6 b. Dynamic height relative to 1000 decibar surface, August 1974 (dyn. m.)

7. Vertical distribution of temperature, section A, August 1974 $\left({ }^{\circ} \mathrm{C}\right)$ 


\section{LIST OF ILLUSTRATIONS-Continued}

Figure

8. Vertical distribution of temperature. section 13. Angrust 1974

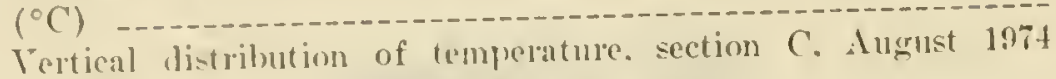

$\left({ }^{\circ} \mathrm{C}\right)$ _

10. Vertical distribution of temperature, section D. Sugnst 1974 $\left({ }^{\circ} \mathrm{C}\right)$ Vortical distribution of temperature, section li. August 1974 $\left({ }^{\circ} \mathrm{C}\right)$ -

12. Vertical distrihution of temperature. section F. Mugnst 1974 $\left({ }^{\circ} \mathrm{C}\right)$

13. Vertical distribution of salinity section A. Angrust $197+(\% / \%)$

14. Tertical distribution of salinity, section 13 . Aurrust $1974(\% / \%)$--

15. Vertical distribution of salinity. section C. \nerust $1974(\%$ oo $)$--

16. Vertical distribution of salinity. section ?). August $197+(\%)$--

17. Vertical distribution of salinity, section E. Iugust 1974 (\% oo --

18. Tertical distribution of salinity. section F. Ausrust $197+(\%)$

19. Vertical distribution of sigma-t. section 1. August 1974 _.....-

20. Vertical distribution of sigma-t, section 13. Angrust 1974 .......-

.21. Vertical distribution of sigma-t, section C. August 1974 _...-.--

22. Vertical distribution of sigma-t, section I). Sugrust 1974 _.....-.

23. Fertical distribution of sigma-t. section E. Iugrust 197t _......-

24. Tertical distribution of sigma-t, section F. August 197t .......

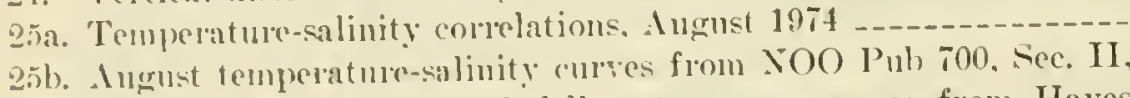
and combined spring and fall watel mass ranges from Hayes (1975)

26. Surface (30 foot depth) current drogne

27. Drogue moxement $2310 \% 11$ Iurust to $1100 \% 12$ Augrust. 1974 ...

28. Droerue movenent $2146 \% 12$ August in $1115 \% 14$ August. 1974 -.-

29. Irerage temperature-salinity colrelations, stations, 49, 50, 51 and 53

\section{LIST OF TABLES}

Table

Pagr

1. STD Euvironmental Profiling System Data Corrections --_---

2. Comparison of oceamoeraphic features in the New York Bight in Angust 1974 with those reported by other investigators --_---

3. Iverage wind duringer drogue tracking 


\title{
OCEANOGRAPHY OF THE NEW YORK BIGHT AUGUST 1974
}

\author{
by \\ Charles W. Morgan ' \\ Joseph M. Bishop ${ }^{2}$ \\ Frank F. Mulher ${ }^{3}$
}

\section{INTRODUCTION}

In oceanographic survey of the waters of the New Tork Bight (Block Island to Cape May) was conducted by the USCGC EVERGREEX during August 197t. The purpose of the cluise was to continue data collection for use in a coastal surface current model to be useal in Seareh ant Resene planning. "The survey, condueted during the period 8-20 August, consisted of six sections laid perpendicular to the trend of the coast between Block Island, Rhote Island and Cape May, New Jelsey (fig. 1). Each section was designed to contain two stations in the slope water beyond the eontinental shelf, one station on the continental slope. and four to five stations on the continental shelf, thus providing information on not only shelf processes. but also on the aljacent slope water. Station spacing was approximately 1.i natical miles, and section sparing was approxinately ti nautical miles. In addition to the oceanographie survey, Alree current meter arrays were deployed south of Long Island (fig. 1).

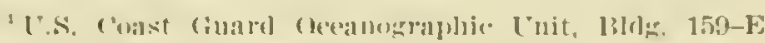

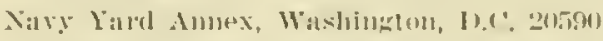

"Now with lowe Watel l'olts l'roject, Envilommental

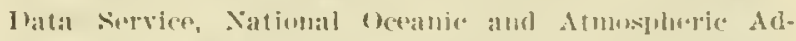
ministratiu, I'age buileling, Washington b.('. 20:35

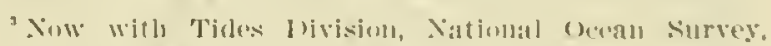
Sational (henaic and Atmospheric Afluinistlation, 6001 Executive Is]vd., Itokville, Marylind 208.i2
} 


\section{PROCEDURES}

\section{Oceanographic Sampling}

At rach staton an s'Th) (salinity-lempela-

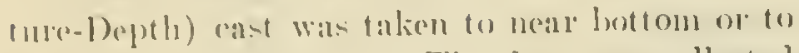
a deptls of 1100 meterts. The data was collected

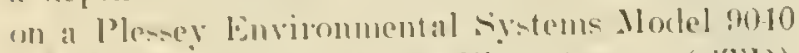
$\therefore$ T 1) bivibumental b'rofiline sir-tem (s'll) (solial mumbler 5i31:3). The data were resorded

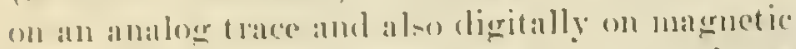
tape. The dienital reseroding was made by a Simeraft Digital Data Lorgere (DDI, mantfactured under ('oast (iuard contract ( $\mathrm{G}-12 \mathrm{z}$ : Tis-1. Four ehamels of information were samplerl at lates of 0.5 or 1.0 scans per second. sTI) fropuencies representing elepth. temperatfure, and silinity were converted to hinary costed deciunl (BCD) and recoleted on a 7 channel l3.11 compatible mannetic tape at a bit clensity of enot hip. The resolution of the DDI, system is tone hert\%. One hertz corresponds to $.00344^{\circ} / 00.018^{\circ} \mathrm{C}$, and 1.90 meters in salinity. temperature, and alepth lespectively. The fourth information channel was arailable for recording semed relocity (me the 1)Dl. hut it was not useel. The tape format for each siln) cast consinted of 3 sets of station data such as station number. position. date. anel time followed by any number of data recorets, depending on the maximum depth and lowering rate of the cast. Fach record consisted of the temperature and salinity information at 100 depth lovels. Thus, an average one thousand meter (alst was composed of alout 1200) dala levels recorted on approximately 120

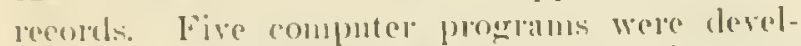

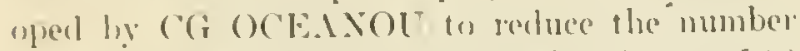
of latia levels to a more matuageable figure of it) 10) 100 data lovels at staudald depths and intlection points which would still acemately represent the origrinal water eolumm.

The computer programs were dereloped for at ('ontrol Data (orporation (CDC) 33300 ('omphrser. I flow diantan of the processing proce-

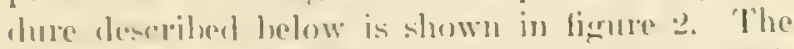

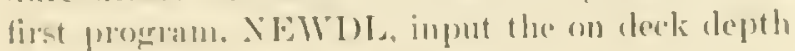
fremeney of the depth sensors. and read the recorels to be processed from the masuetic tape. The digitized frequencies were translated from 13('D) (1) enerinecring mits of deptlo (meters to tenths), temperature (C ${ }^{\circ}$ to humbledths), and salinity (\% to humbledths). The values were printerl out so that an initial eheck of the data rould he mate. In addition. a lape output

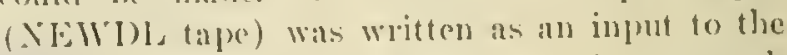
next prorratu. With a rapid sample rate such ats 0.5 second. a spercific depth level might show 11) - aceral times. While these temperature and silinity values were always close, they generally dicl not andee exactly, plobsalsy as a result of solusur lag. The output from the first program was mormally anomit $1: 200$ levels of data for a 1000 meter cist.

Plospam AVCOR averaged data levels inputed from the Nowlol, tape at the same depth level. ATrOR acepterl sedfuential levels untjl a decper level was reached: then it bearan the averatring for the next level. Therefore if, due to the ship rollinge the stTy dips to a lower level and then refurns of the origumal level, the data at the origrinal level subsequent to the roll will not he inclurled in the arerage. During the Ale(o) proressing, corrections are male to temperature and salinify as discussed in the following section. The output of $A T C O R$ is a printout and a magnetic tape ( $\mathrm{MVCOR}$ tape). The printout of tomperature. salinity and computed sigmat was quality controlled lỵ remoring samples which anded averaged sigma-t ralues to decrease more than 0.2. 0.05, or 0.02 per averatered data level within 0-100 meters, 100-300 meters, and deeper than 300 meters respectively.

Use of these criteria oreasionally permitted data to pass which indicated lange instabitities in the water columm, as revealed ly computation of the stability or $\mathrm{E}$ value (sirerliup, et al. 1942 .

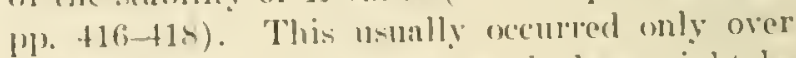
small intervals. (Athomgh such data might be furentionerl. the data las not been rejected: this will permit other investigators to dran thejr own munchusions as to whether or not to use the data. 
All clata has been used in the analysis presented in this report.)

Procran FINAV, which input the ATCOR tape, reaveraged the data after data levels which failed to pass the IVCOR sigma-t test were removed. 'The ontput of FINAT is a printout and a computer eard deck. 'The FIXIV printont. Was quality controlled by rechecking the sigma-t values to ascertain the elleet of the clata level deletions on the FIS.IV rmo. For varions: reasons. the zero meter depth level is not recorded by the DDI. Kero level data is olstained from the s'TI trace or extrapolation, and entered into the computer card deck.

The fourth trogram, sIGPT, determined the standard and significant levels, whose temperature and salinity would accurately represent the original water column. Standard levels were taken at the depths falling closest to minimum recorded tepth, 10, 20,30, 50, 75, and 100 meters, every 2.5 meters to 300 meters, and then every 50 meters to 1000 meters. The first test for sirnificant levels consisted of fitting a cubic curve through five consecutive temperature lata points. If the curvature at the midpoint exceeted an absolute rahe of $0.00 \%$. the seeond, third. and fomth points were compared with the data points immediately above and below. A level was significant if it departed from a straight line between the adjacent points by more than $0.0-1^{\circ} \mathrm{C}$ for temperature (more than $0.06^{\circ}$ o for salinity). The second test compared the ditlerenees hetween the curvature of two sucessive midpoints. If the absolute value of the difference exceeded 0.00 \%, the departure of the point from the adjacent points was again checked, using the same limits as in the first test to determine if the point was significant. If both of these tests were negative, the departure of levels from points immediately above and below was arain checkel. If the absolute departure was wreater than 0.09 for both temperature and salinity, the level was significant. If the limits were not exceeded in any of the three tests, the level was not significant. After rmming the same checks for salinity, the top level of the five level group was dropped and the next new level was adderl onto the bottom end, and the testing was legun again. The ontput of SIGI'T was a printont and computer card sleck. The printout was cherker] for obrious errors such as wronger input.
The final program. SARCS, plots temperature, salinjer. and simna-t rersus depth, and also plots a 'T-s diarram. The ontur, in acllition to the plots, consists of a printont and computer card derek. The printout was suljecter] to a final quality control hased on a careful study of the plots which indicated that the data reported herein was not grossly unreasonalyle. The card deck was sulmutted to NOI) ( Note: Recent changes fo standarlize the data processing procechres at the ('G Oceanographic Lnit have resulted in some program namo phanges as well as minor changes in the way in which future lata will he processed.)

\section{Quality Control}

STD data were quality controlled hy comparing s'l' amalow trace and DDI, values with temperature and salinity values olstained from Niskin bottles attaclied just above the underwater sensor unit. Quality control (QC) samples were taken at the suface. 200 , 500, and 1000 meter's where possible. The Niskin bottle was equipped with poteded (and for the 500 and 1000 meter samples, mprotectel) (leep sea rerersing themometers. 'The thermoneters were allowed to soak for six minutes at each QC depth to reach equilihrim before the Niskin bottle was tripped. The conductivity atios of the quality control samples were determined ring an induetive laboratory salinometer and were converted to salinities ntilizing the methot established in the International Oceanomaphice Tables pullished jointly by UTESCO and the National Institute of Occanomuphy of Great Britain (1966).

The diflerence between STT and quality control values of temperature, salinity, and depth were plotted anainst the station numbers in the orler in which they were ocempjed. Inspection of the plots indicated that the depth and temperature values should be corrected by values which dict not change throughout the eruise. The eorrection for salinity values appeared to aro through thres phases, hecoming worse as the eruise progresserl. The final corrections shown in Table 1 wore based on the aremare corrections for surface and 1000 moters. 'The correction for' intermediate values was linearly interpolated. The data for 200 and 500 meters indicated that 
TABLE 1.-STD Environmental Profiling System Data Corrections

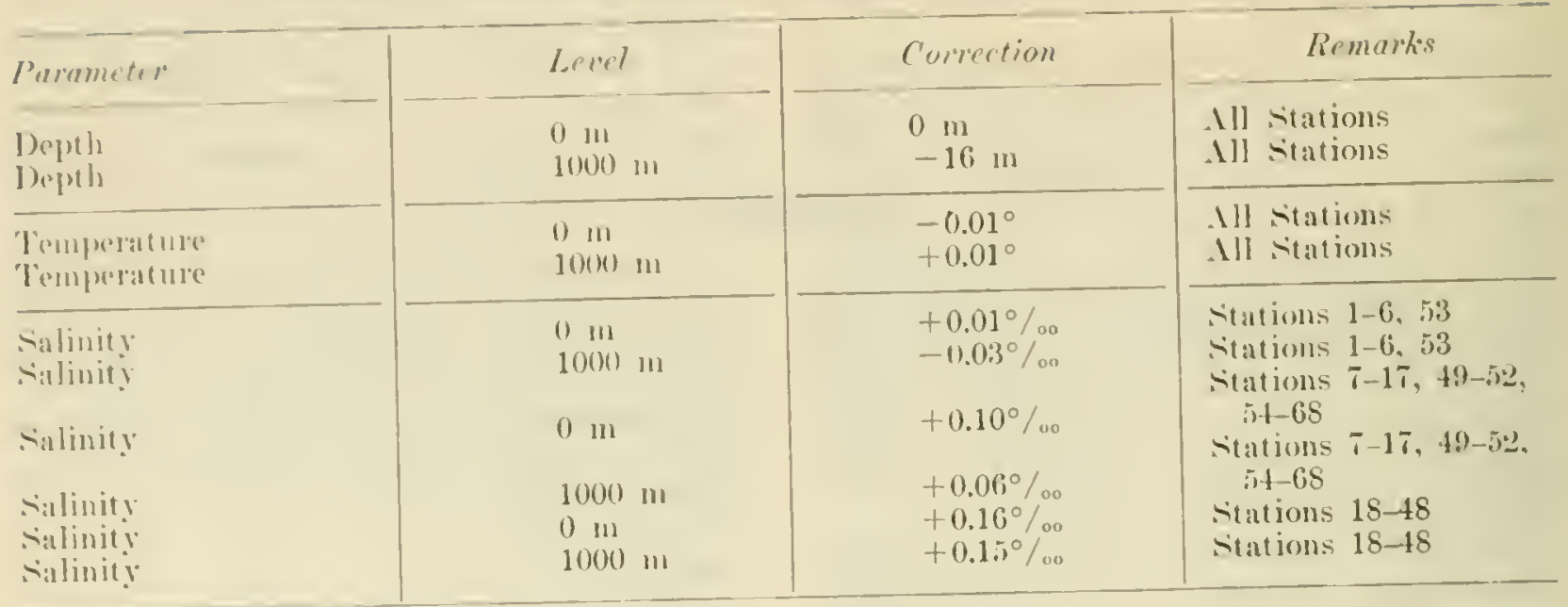

the actual correction shonld not have been linear: howerer, the 200 and itno meter data did not secun suflicient to justify a more complex correction.

\section{Navigation}

Navigation during the eruise was hased primarily on information from Ioran-C. Joran-A. fathometer, satellite navigation ( $\mathrm{X} \perp \mathrm{SAT}$ ), and 0.11 lifi.l were noed as backup systens. Positions on most of the cruise were probably ac(urate to ().2-0.t mini.

\section{Current Meters}

Three current meter arrays were set for a period of about 2 weeks sonth of Jong Island (fig. 1). Array $\# 1$ consisterl of a current meter at approximately 20 meters; aray $\# 2$ consisted of currem meters at apploximately 20 and 40 meters; and array $\# 3$ comsisted of a current motes at approximately 20 meters. The data from these current meters an now heing analyzed and the results of the analysis are to be reported in a future publication by the Oceanographic Unit. 


\section{DATA PRESENTATION}

\section{Data Listing}

Temperature. salinity. and deptl values at stamblard levels of (0, 10). 2(1), 30, 40, 50, 75, 100. 150. 200, 2.6), 300. $4(00) .500,600,700,800,900$. 1000, 1100, 1:00, 1300, 1400 and 1500 meters. along with time. position, meteomological. and sca surface clata were sulmotted to the Sational Oceanographic Data ('onter (XOI)C). which later provicled printenl data listiness. In adclition to the data submitted. the printed listings also contain values for sieruat. specific volume anomaly, dymatc lobight, and sound velocity

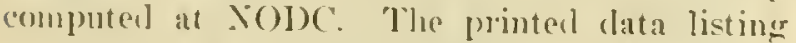
for this cruise is contained in Ippendix 1.

\section{Surface Contours}

siurface values of temperature and salinity were plotted along the cruise track, and surface contours were produred from these values (figs. 3 and 4). The sea surface temperature contours from the cruise may be compared to those collected 19-21 Augrust 107t during a Coast Guard Airborne Radiation Thermometer flight (fir. i).

\section{Mean Vertically Averaged Sigma-t,}

Column averaged values for sirnat on the whelf were computed using the-finite difference relationsurip

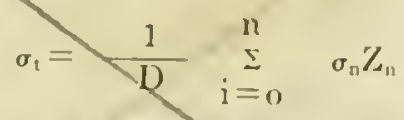

where $\sigma_{n}=\left(\sigma_{t}+\sigma_{B}\right) / 2$ is the mean value of signat in liyer of thicliness $Z_{n}, \sigma_{2}$ and $\sigma_{B}$ al' ( the sigmat values at the top and bottom of the layer respectively, and I) the depth of the deepest olservation. not to exceed 200011 . Contours of mean rertically areraged sigmty-t (fig. 6a) -een to be linked to the genalyt stimmer cireulation battern- which appears to parallel the coast (Вแ111им. 1969).

\section{Dynamic Height Contours}

The greneral surface circulation along the eastcrn continental slope can be inferred from dymanur height contours (fig. 6h). Flow is paralle? to the isopleths with high values to the right Jooking downstream. The assumptions and theory of inferring currents from dymanic heights are discussed in sierdrup ot al. (194?. ly. 451-i5i). Dynamic heights were referenced to the 1000 decibar level. The reforence level was chosen using the methor of Defant (1941). I) Ynamic bejglits for stations where the water. deptli was less than 1000 meters were caleulated in a manner similar to that described by MellandIanien (1934).

'ilhe general pattern shown hy the dynamic topography chart is a 10 to $30^{\circ} \mathrm{cm} \mathrm{s}^{1-}$ sonthwesterly How on the sholf and two anticyclonic circulations in the slope water. The soitherly of the two circulations is obvionsly an eddy. Infrared satellite imasery subsequent to the cruise leaves little dould that the noltherly circulation is also an arloty.

\section{Vertical Section Contours}

Vertical sections for temperature. salinity, and sirma-t to a depth of 1000 meters were drawn for fiections 1 -I" which were approximately nomal to the coastline (fiers. - i-24). I more meaningful presontation of rertical section eontours was proluced by greatly axacrerating the vertical distance scale in comparison to the horizontal distance seale. 


\section{RESULTS}

The unnual eycles of temperature and salinity on the continental shelf hetween Cape Cod and ('ape Ilatteras lane been described by Bigelow (1933). Bigelow and wears (1935), ITalfort and
Wicklund (1968). and others. The conditions found in August 197t were in general agreement with most features found by previous investigators (Table:2).

\section{TABLE 2.-Comparison of Oceanographic Features in the New York Bight in August 1974 With Those Reported by Other Investigators}

\section{Iisture}

Sica surface temperature

Temperature ditforence between surface and bot tom at :35-50

meter eontour zone

Sea surfuce salinity

presence of cold rore on shelf

l'renence of high salinity core on slope

Presence of shelf/slope temperature front

I'resence of shelf/slope saliuity front
Alugust $197^{4}$

$20^{\circ}$ to $25^{\circ} \mathrm{C}$

$9^{\circ} 1015^{\circ} \mathrm{C}$
Other Investigators

$20^{\circ}$ to $25^{\circ} \mathrm{C}$

(Wriford and Wicklund, 1968)

13 to $16^{\circ} \mathrm{C}$

(Bigrelow, 1933)

$15^{\circ} \mathrm{C}$

(Walford and Wicklund)

$<31 \%$ to $>35 \%<32 \%$ to $>35 \%$

(Bigelow and Sears, 1935)

Yes Yes (Bigelow, 1933;

Bigrelow and sears, 1935 ;

Whitcomb, 1970)

les

Yes (Bigelow and Sears, 1 !)35; Whitcomb, 1970)

les Ves (Bigelow, 1933)

Bigclow and Sears, 1935 ; Cresswell, 1967)

Jes les (IBigelow, 1933;

Bigelow and Nears,

1935; Cresswell, 1967)

\section{Cold Core}

A cold core was found on the shelf at depths of $20 \mathrm{~m}$. to $60 \mathrm{~m}$. from the surface, at a distance of 20 to $70 \mathrm{mmi}$ from the coast (fig. $\overline{-}-\mathbf{1} 2$ ). This core, mentioned by Bigrelow (1933), was defined by Whitcomb (19\%0). as laving temperatures below $8{ }^{\circ}($. The pool or rore is the remant of a winter shelf water formed at the surface (Whiteomb, 1970). Because of the southwesterly 0.2-().5 nui per day bothom drift along the shelf (13umpus, 19)(6i)). Hhere is some renewal of the core from the northeast, howerere, this renewal is probably minor compares to the annual renewal through surface cooling. The core, in
August 1974. was found only at stations 11 and 21. thus it was consilerably smaller than that shown in Whitcomb $(1970)$ and than the $7.5^{\circ} \mathrm{C}$ core shown in Walford and Wicklund (1968). However, the presence of a cold core lefined by the $10^{\circ} \mathrm{C}$ isotheru can he easily traced along the shrelf from section $F$ to section $A$ (firs. $i-12$ ). Evidenes of a tongre related to the core was found at station 28. The source of this tongue an be traced northeast thromgh station 37 to station ti) along the sigma-t. surface of about 26.0. In altermative identification of a tongue as " "alvol lubble" is discussed hy Cresswell (1967). 


\section{High Salinity Core of Slope Water}

Extending parallel to the shelf edge, und 5 to 10 nautical miles seawarl from this edge. was a band of higher salinity water similar to that reported by Bigelow and sears (1935) and others [Whitcomb (1970) for example]. 'This hand is simply an expression of the impingement on the slope bottom of typrical North Atlantic Central Water (Iselin, 1936), the surface of which has been freshed by mixing with shelf water. Following Whitcomb's (1970) exanple for Scptember 1967 of defining the core as salinities greater than $35.75 \%$ oo, the defined core did not reach the surface, and its depth range was dependent on whether or not there was an eddy present.

On section i the core was characterized by an anticyclonic eddy which caused the crosssection of this core to increase considerably. Maximum salinity in the core section of this eddy was $36.2 \%$, and the $35.75 \%$ isohaline extended from about 20 to 375 meters. 'The definerl core was absent on section $B$ north of the eddy; on sections $\mathrm{C}$ and $\mathrm{D}$ it was found between about 70 to 120 meters. Sections $E$ and $F$ were inflnenced by a large eddy eastward of the sections, thus the defined core extended from about 30 to 210 meters and was still increasing in thickness at the end of the sections. The salinity and sigma-t profiles show little evidence for the $35.75 \%$ core intersecting the bottom, although there is an obvious bottom salinity maximum orer the shelf break.

\section{Temperature/Salinity Correlations}

The temperature salinity correlation for water present in the Yew York Bight during August 1974 could be accounted for in terms of the principal modes described by Hayes (1975) (figs. 25a, and $25 \mathrm{~b})$.

In August 1974 waters from the coastal area and contained within a band extending approximately 40 nautical miles offshore had characteristics that fell within an envelope with salinities less than $33.5 \%$ (Envelope $\Lambda$, fig. 25a). Tote that the lower portion of this envelope includes what Hayes called Middle Atlantic Bight Coastal Water. The lower portion of the envelope also represents the cold core previously describer. The upper portion of the envelope reflects the warming etfect of summer surface heating and the freshening effect of spring lunoff.
Water from the centers of the two eddies fell within an envelope with salinities greater than $34.0 \%$ (Envelope 13, fig. 25a). displaying characteristics sinilar to those described as Regions 8 and 9 in "Physical Properties of the North Atlantic Ocean," Naval Ocemograplic Oflice Publication \#700, Section II (fig. 25h). This envelope could also be explained in terms of Hayes, Gulf Stream Water. Sinface-and Midslope Water, and Deep Slope/North Atlantic Decp Water if allowance were made for summer warming of his Sinface-and Mid-slope Water (fig. 2.5b).

It the stations between those found in the two envelopes the water slows the influence of mixing between the envelopes. Station 28 (figr. 25a) is an extreme example of this mixing. The water at the surface shows characteristies similar 10 that in envelope 13 ; at depths of about 2.5 to 70 meters water derivel from the cold core is encountered, helow this the midl-slope water is found. An example of this type of mixing in shallower shelf water can be seen at station 12 (fig. 25a). Here the influence of surface water in envelope $I$ is much stronger than that in envelope B. Another example of this type of mixing, in deeper slope water, can be observed at station 53 (figr. 25:a). Ilere the influence of sunface water from anvelope 1 cannot be seen at all, and the influence of the cold low salinity core at the bottom of envelope $A$ is slight. Similar situations are found for stations $6,51,50,54$, 55, and 4,9 around the southern eddy, and for station 26 near the northern eddy. These stations appear to basically represent slope or edly water with which some shelf water has been mixed.

Station 29 on the shelf represents intrusion of slope and eddy derived water onto the shelf. This is apparent in the salinilies of $35.5 \%$ found around 30 meters.

\section{Circulation}

In coastal waters, where there is adequate fresh water clischarge, a slope of the sea surface downward from the coast offshore is usually attriluted to the increases in the steric anomaly related to run-off. The resulting dynamic gradient is associated with a steady flow turned to the right (in the northern lemisphere) and thus nearly parallel to the coast. Steady wind drift currents may modify this rough picture (Bum- 
pus. 19k:?). In a lecent ('on-t fiundel Oceano-

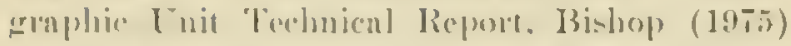
develope an operationally oricnted technique to

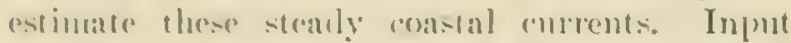
paraneless to the model are the surface wind stress and mean redtically-abelaged sigma-t Fralient.

On the Angust 107t clutice, measumements of

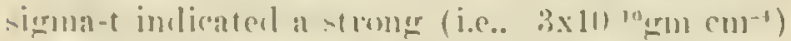
ron-shelf grationt in the vertically averared

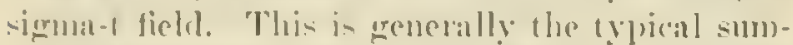
mer alemsity strueture a- contanted to the weakly

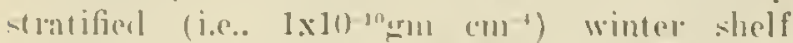
water: The trem values of the order of $10^{2}$ dymes/em-2 foward the notheast while winter mean stress is in the 1 dyne (rot" lange towand the soutliwest accorelinir to data the for io syuare centered at

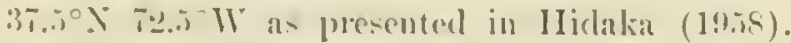
lioth in summer and winter as soutls to south.

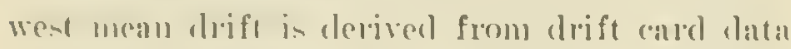
(13mupus. 1969). It seems straight forwarl that this rolocity field (approximately erpua] in marnitule for anch reasom) is maintained in the slimuer montlos ly he well developed density tiold. and in the winter he the mean wind stress.

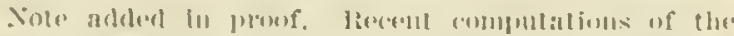

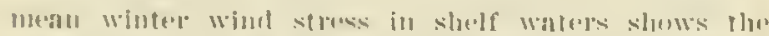

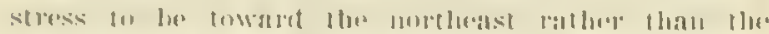

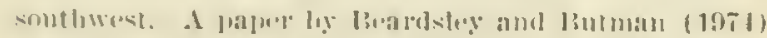

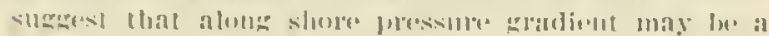

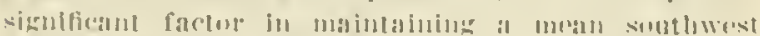

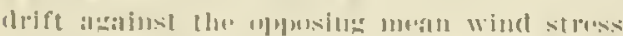

Measmed ralues of this mean vertically areme aged sigmat pralient, obtained on this eruise. were necrl in eakenlations to atimate surface cosatal dyift laserl on the above mentioned analytical molel (wimbl stress was norlected). The result indicated a shelf cirembtion (tier. lia) frenerally setting towat the solthwest with maximum smfare velorities neat the thelf loreak of alpurosimately of 2011 all sece. This alalenlation

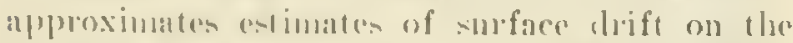

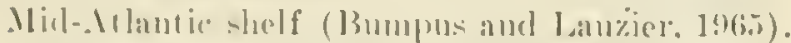

Comparion of the shelf cireulation derived from this moxlel (lige fia) with that derived from

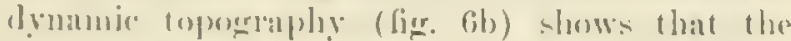

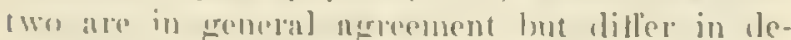

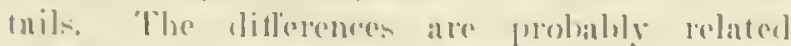
equally 60 diflerences in lle eroverninger equations (Bishop inclutes friction in his model) and to diflerenees in upplying the data (Bishop) uses a mean sigmat ermdient for each section: the dymanie methorl naes the druanuic height for indiviclual stations).

In waters seawarl of the slope. contours of dynanice lopights referemeed to 1000 meters (figr. 6i) indicate the presence of two anticyelonic echlies with a trough between them. The slope ritculation is dominated ly the two edelies. the only uther feature presom loeing the tromerh. Maximm arostophic spreels in the southern eddy atre approximately do (om see

\section{In Anticyclonic Fddy in the Slope Water}

One of the interesting features found during this artuine wats the anticyclenic eddy located alout 115 mmi smothwest of (ape May. Vew Jersey (fig. (ib). Eildies such as this are a common foature in the slope water alone the contiuental slope of the Xew York 1Bierht. Infrared satcllite imarery slows that there is a continual progresion of steh anticyolonir edclies through the Jight. 'They e(momomly have a dianeter of 50 to 110 mui with a spaciug of about 110 to 220 mon letweren eslalies. The aldies seem to form from mennders in the Forth Atlantic Current in the northwest Alantice generally enst of $6.3^{\circ} \mathrm{W}$. and from there drift westwarel and soutlewestwarlalong the continental slope until they reach the ricinity of Cape Hatteras where they rejoin lle Gulf situram (figr.

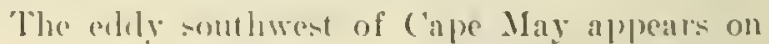
lhe temulerature. stlinity. and density sections as a core of warm saline water which is less leise than the suroumding water (fiers. $i, 13$. and 19). This corre has a tomperature of $15^{\circ} 10$ $16{ }^{\circ} \mathrm{C}$, a solinity of 36.1 to $36.2 \%$ "10. and a $\sigma_{6}$ of $2(i$, Sol to 27.0$)$.

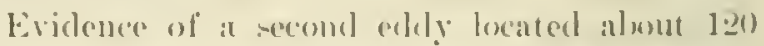
umi soutle of Bloxle Inland was fomol on sections F: and If (fir. (i). The enter of the northern

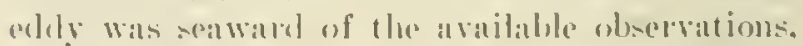
and no conclusions an he drawn comparing the two edilies.

The cirrulation patforn aloumd both eddies was anticyelonice, as inclicater on the dyanume toporraphy ahat. The dynamie topongaphy

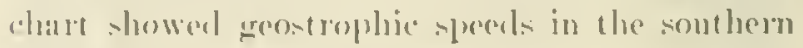

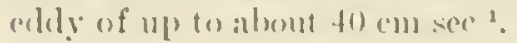

Following the survey of the smaller eddy, a surface current drourue (fir. 26) was deployed 
in the sonthwest quadrant of the eddy and tracked by IOR.IN C for 12 hours (fig. 27). The drogne was then recovered and re-deployed in the eddy's northern quadrant and tracked for about 36 hours (fig. 28). The tracks of the drogre can be accounted for satisfactorily by assuming that prior to and during the drogue experinent the eddy drifted sonthwar at a speed of about 0.13 knots. and that the current acting on the drogue was the rector sum of the greostrophic flow in the eddy and a simple wind driven enrent as described in the Sational Search and Rescue Manual (1973). The estimated arerage winds for the tracking episodes are shown in Table 3. The effect of inertial currents can be seen in both of the drogue tracks. During the end of the eddy surrey a storm was in progress with winds from the northeast quadlant of the compass at 20 to 25 knots. At about $1600 \%$ on 11 August the wind dropped to 15 linots. This would have permitted an inertial current to begin rotating. The inertial period at the latitude of the eddy is 19.5 hours. It appears from ficrure 27 that the majority of the 12 hour drift of the southwest quadrant drogue track occurred predominantly sluring the portions of the incrtial period in which there was a northward component to the inertial current. This woukl account for the northward displacement of the drogue after 12 hours relative to the position indicated by the combination of wind and geostrophic current. The second drogue track (fig. 28) indicates that when the drogue was launched the inertial current was flowing witl a northwestward component. The westward movement of the lrogue about one inertial period later (1600Z on $13 \mathrm{August}$ ) supports this drogue from the direct track between $2146 Z$ on 12 August and $1600 \mathrm{Z}$ on 13 August represents the diameter of the inertial circle, one can calculate that the inertial velocity was $46 \mathrm{~cm} \mathrm{sec}^{-1}$ (Neumann and Pierson, $1966 ;$ p. 158). A simila. calculation on the drift from $1600 \%$ on 13 August. to $1115 Z$ on 14 August when the drogrue was recovered indicates that the inertial current had decreased to 38 cull ser-1. These speeds anree with inertial speeds griven by Pollarl and Millard $(1970)$.

It is of interest to speculate on the etlect of edclies such as this in cxchangring water between the slope and shelf areas. The avernge T-S characteristics above 30 meter's of stations on the southwest side of the edly are warner and more saline than those on the northeast (fig. 2!). This leads to the hypothesis that the anticyclonic eddies are a contributing factor in the mixing of shelf and slope waters. Inother illustration of possible eldy-related mixing in process can he seen in the salinity profilu of section 1 (fig. 13). The tongure of high salinity water found between 10 and 30 meters on stations 4 and 5 suggests that an eddy can cause intrusion of slope water onto the shelf.

\section{TABLE 3.-Average Wind During Drogue Tracking}

\begin{tabular}{|c|c|c|c|}
\hline Date & Time (Z) & $\operatorname{Dir}\left({ }^{\circ} T\right)$ & spd (kts) \\
\hline Ang 09 & 1800 & 190 & 8 \\
\hline \multirow[t]{4}{*}{ Aug 10} & 0000 & 345 & $T$ \\
\hline & 0600 & 0.7 & 22 \\
\hline & 1200 & 017 & 26 \\
\hline & 1800 & 015 & 27 \\
\hline \multirow[t]{4}{*}{ Aug 11} & 0000 & 026 & 22 \\
\hline & 0600 & 015 & $2: 2$ \\
\hline & 1200 & $04: 2$ & 23 \\
\hline & 1800 & 020 & 14 \\
\hline \multirow[t]{4}{*}{$\operatorname{Lng} 12$} & 0000 & 025 & 17 \\
\hline & 0600 & 017 & 12 \\
\hline & 1200 & 357 & 12 \\
\hline & 1800 & 000 & 13 \\
\hline \multirow[t]{4}{*}{ Lug 13} & 0000 & 325 & 17 \\
\hline & 0600 & 300 & 15 \\
\hline & 1200 & 315 & 15 \\
\hline & 1800 & 260 & 9 \\
\hline \multirow[t]{3}{*}{ Ang 14} & 0000 & 235 & 10 \\
\hline & 0600 & 242 & $\tau$ \\
\hline & 1200 & 235 & 10 \\
\hline
\end{tabular}




\section{REFERENCES}

Beardsley, 12. C. anc] [3. Butman (19T4). ('imulathu

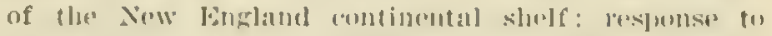

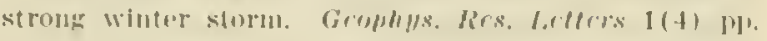
$181-18.1$.

J3igelow; 11. I3. (1933). Stuclies uf the waters un the

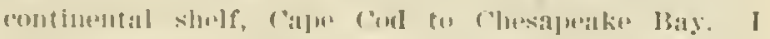

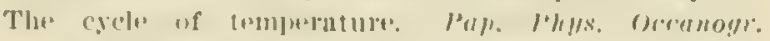
Hetrorol. $2141: 13: 514$.

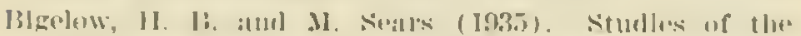

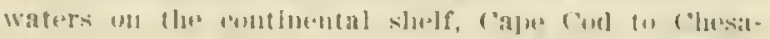

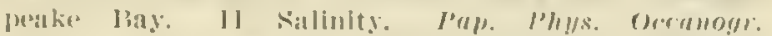

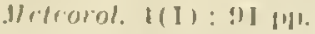

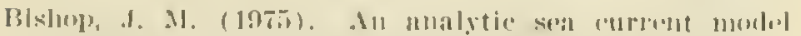

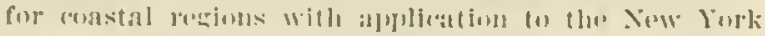

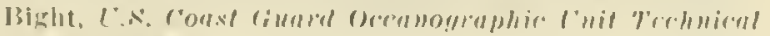
Rrport $7.5-$ ?.

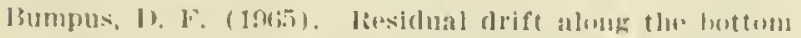

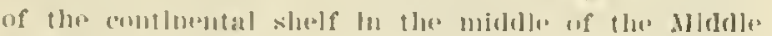

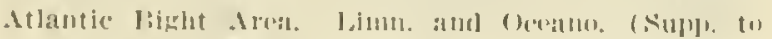

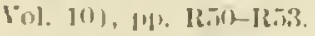

limmpus, 1). I\%. (1!)i!)), lieversals in the surface drift

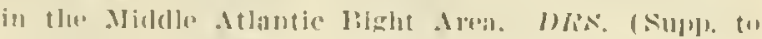

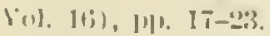

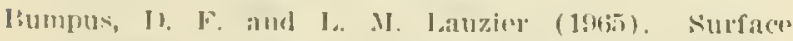

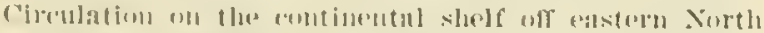
Anerica hetweren Sewfoumdland and Flurida, imeri-

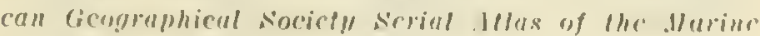
linviroument (lidin T).

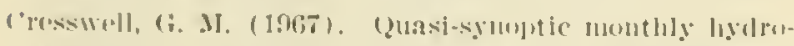

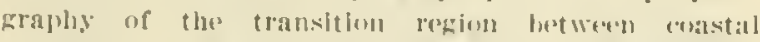

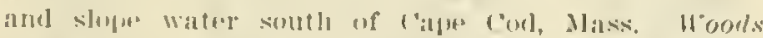

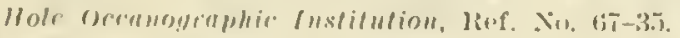

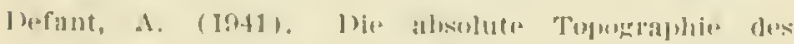

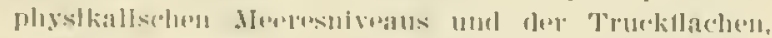

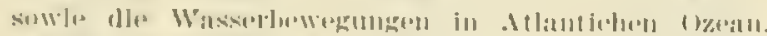

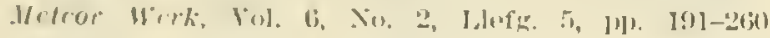
$($ Iserlin).

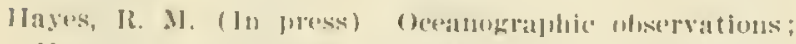

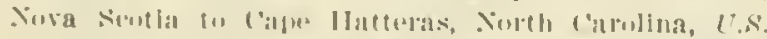

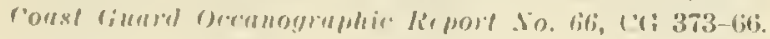

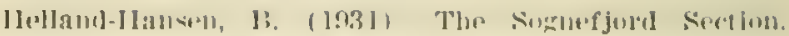

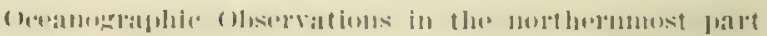

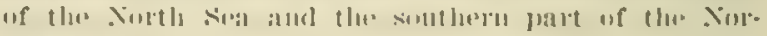

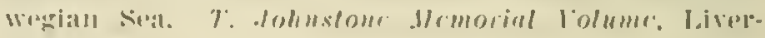
f(1)1, 1!4:31.

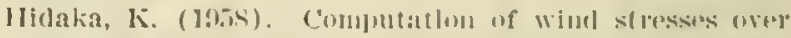

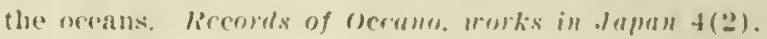
pp. $\div-123$.

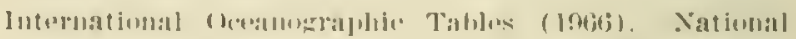

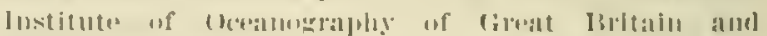

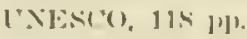

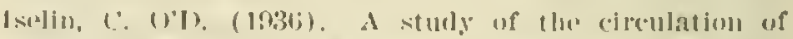

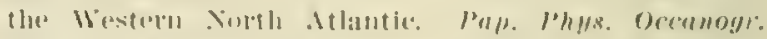

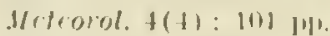

lítelum, B. II, and X. ('orwin (196-1). Tlue prerslstence of "Winter" water on the continental sluedf south of

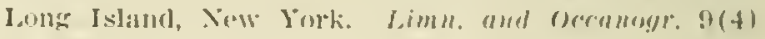
11]). $16 i-175$.

Xemunant, (i. and W. J. I'iersulu (1966). Principles of

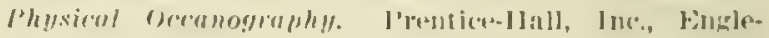
murl ("lifr, X.u.

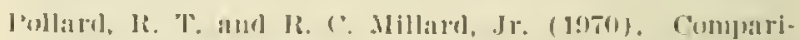

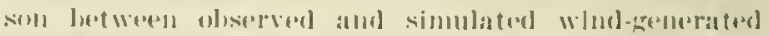

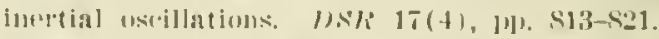

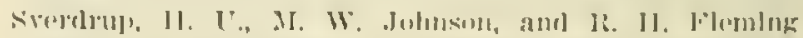

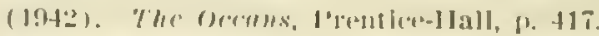

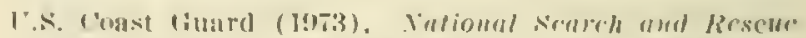

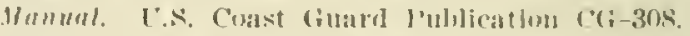

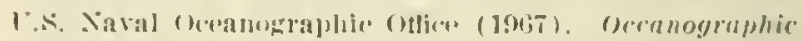

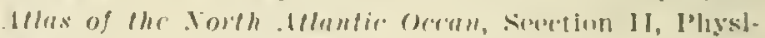
(a) l'rupurties, I'uls, ton.

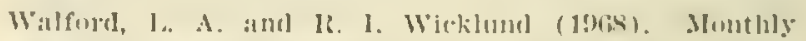

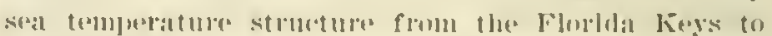

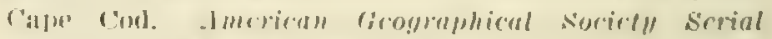
.llas of the llarime Emromment (Folio 15).

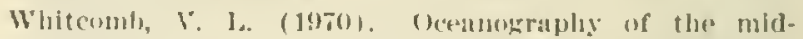

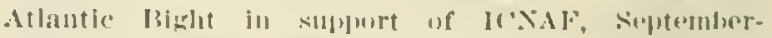

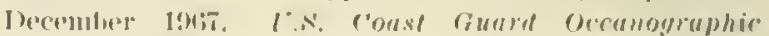
licporf .5o. 35. C1: 373-35. 


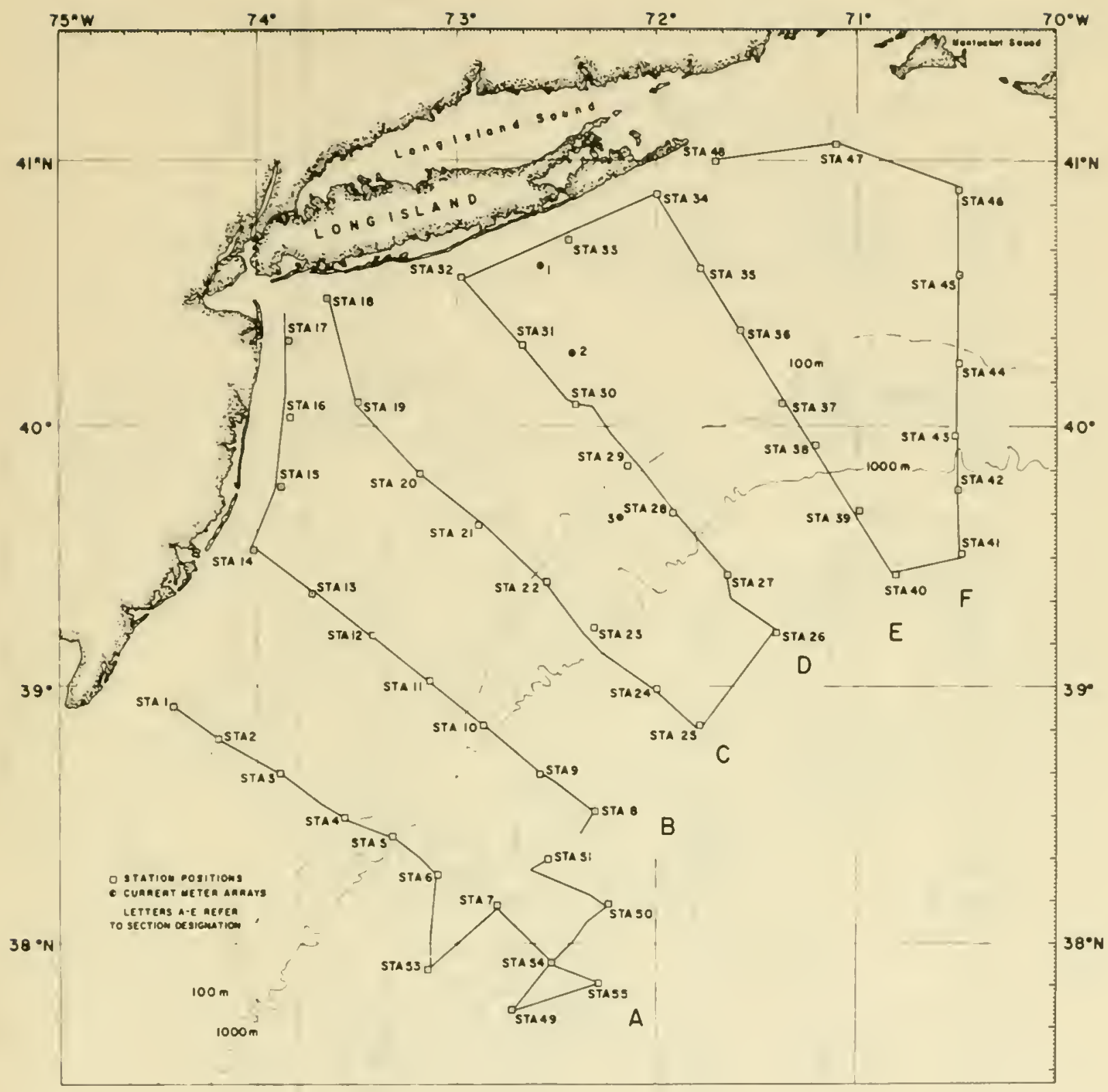

Figur: 1.- Station and section locations, August 1974 


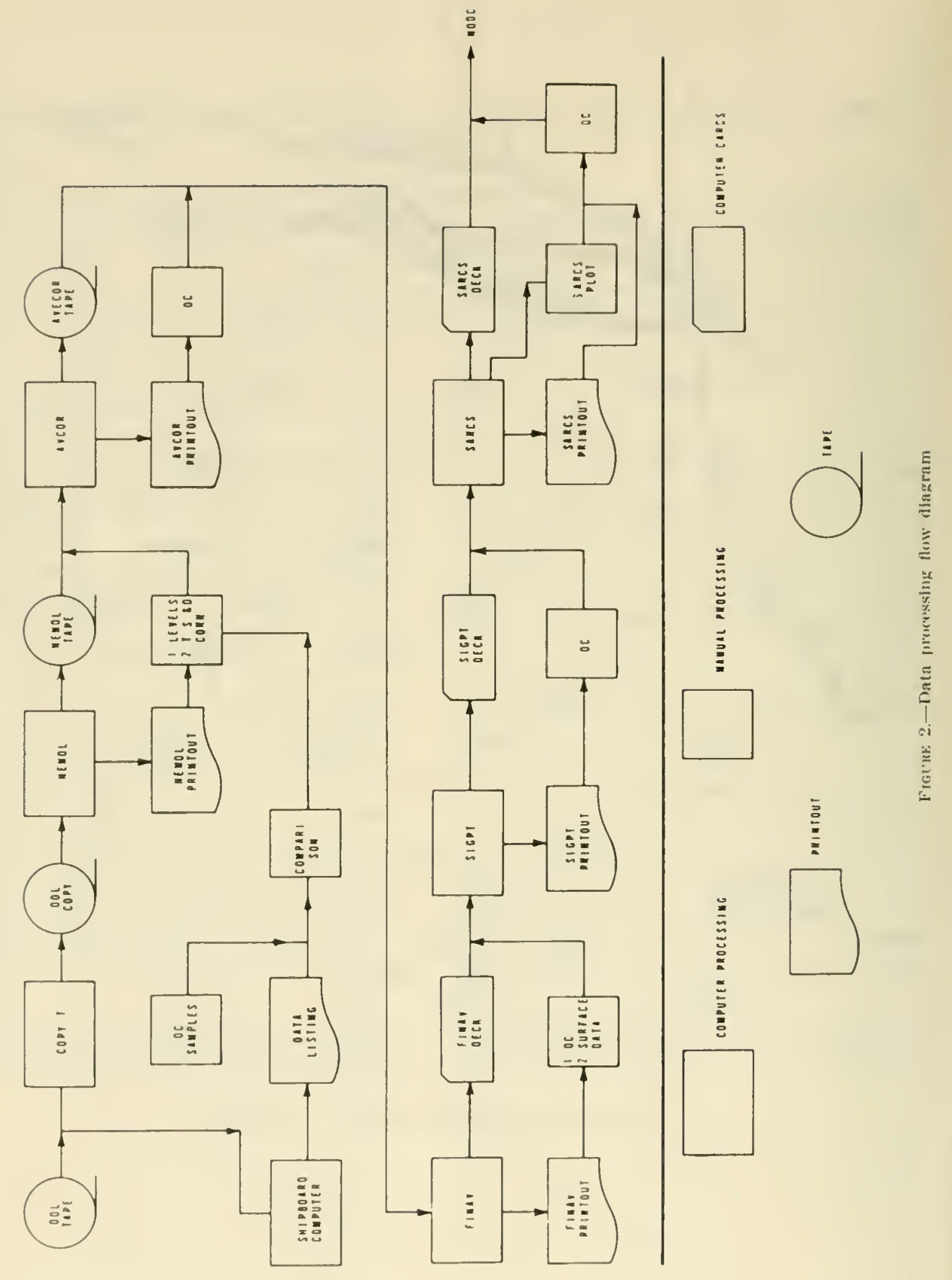




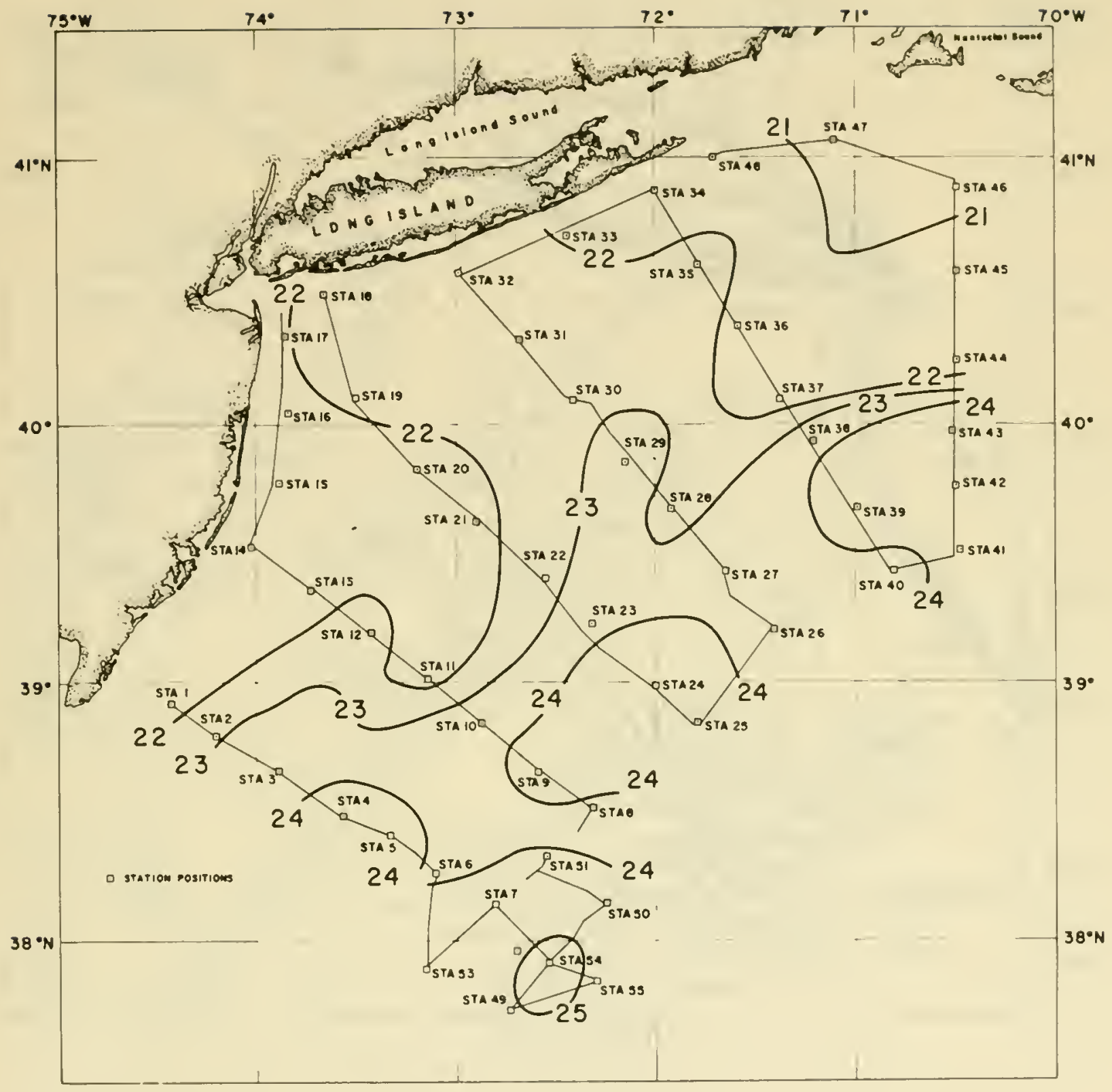

FIGURE 3.-Sea surface temprerature distribution, S-20 August $1974\left({ }^{\circ} \mathrm{C}\right)$ 


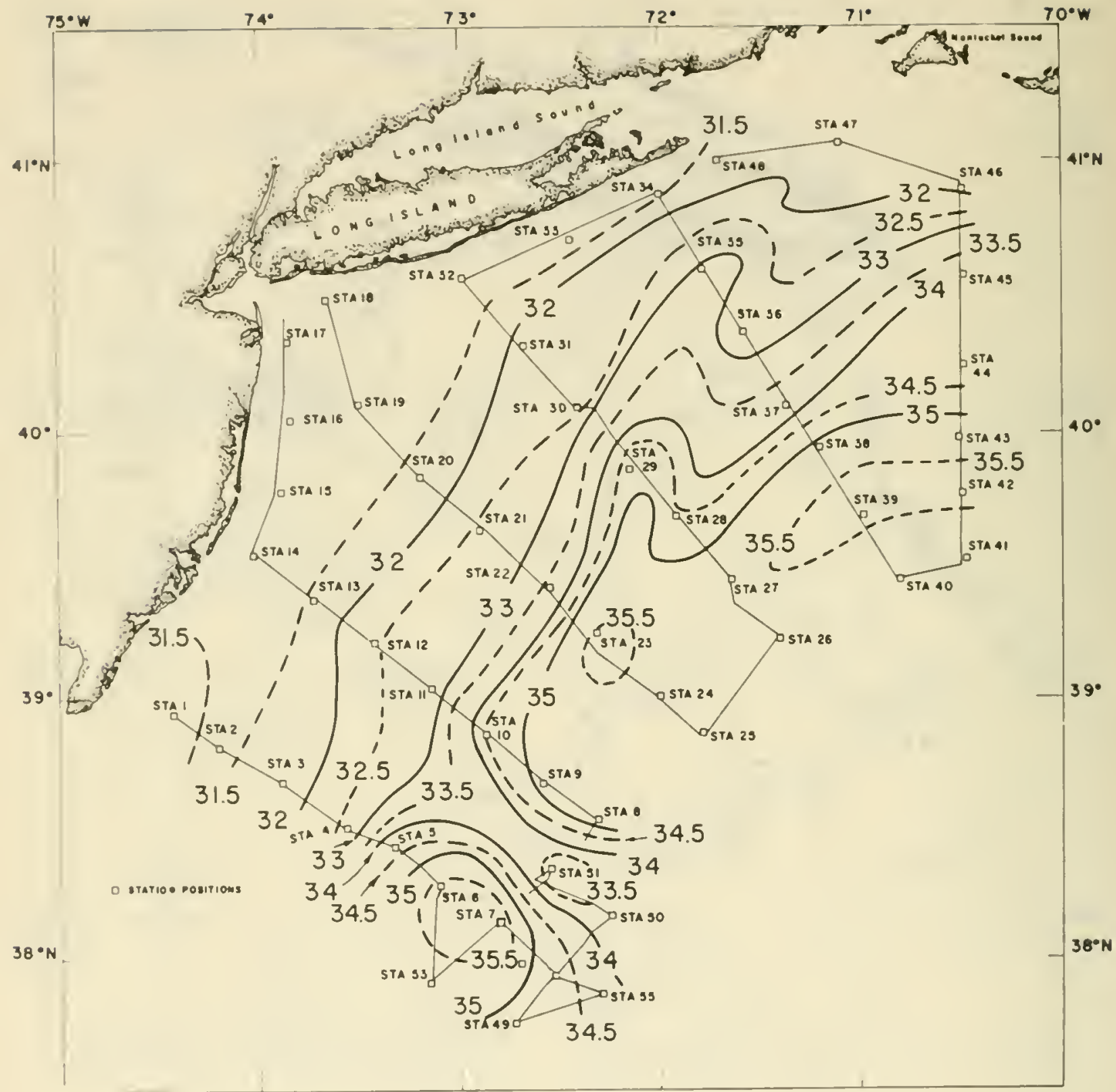

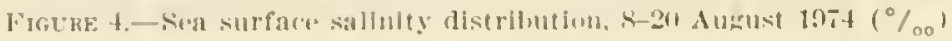




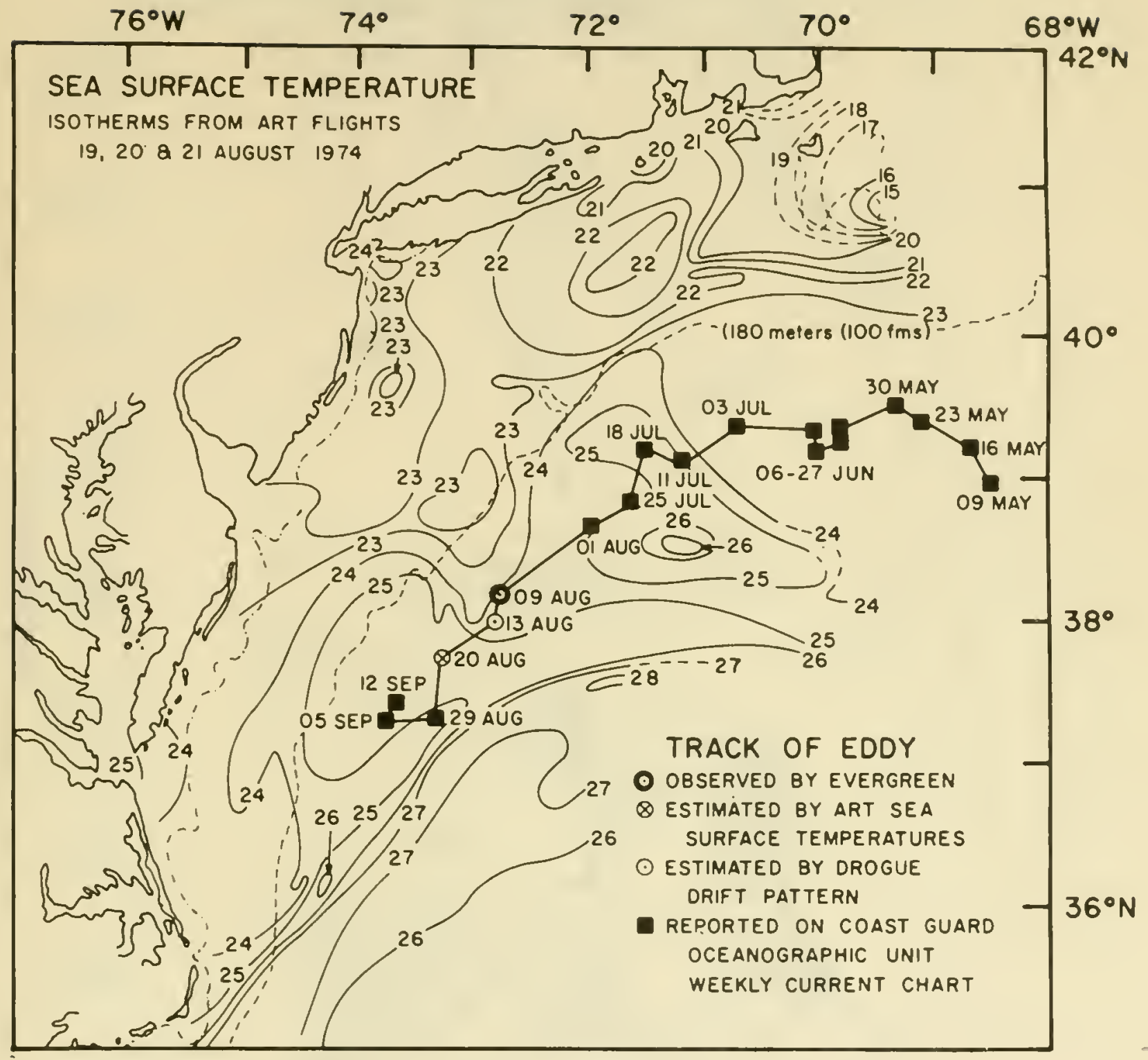

Figure 5.-Sea surface tempratures from August 1974 AR'T flight: track of eddy $\left({ }^{\circ} \mathrm{C}\right)$ 


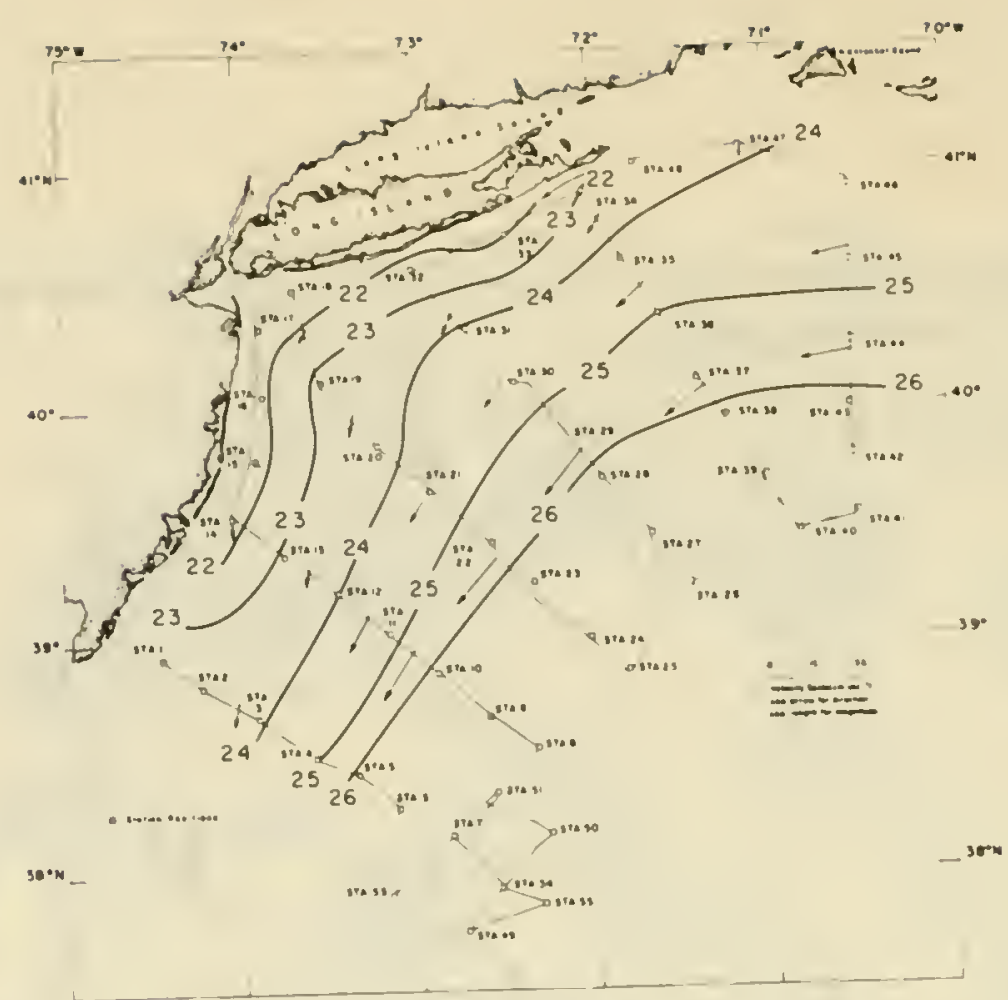

Frgurf, 6a.-Mean vertically-averaged sigma-t, August 1974. (Arrows show current computed from Hishos, 1975)

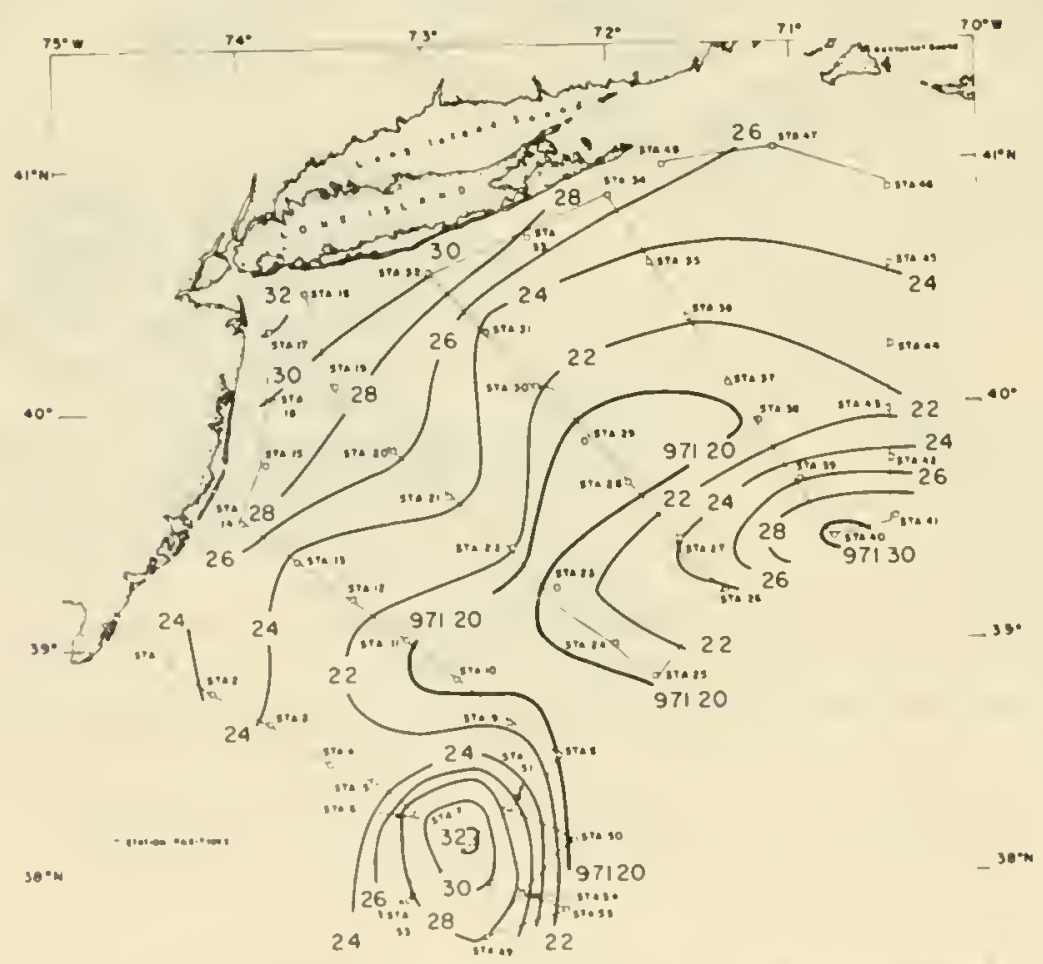

Ficrove 6u-Dinanic height reiative to $1 \mathrm{~mm}$ decihas surfaco.

Angust $197+2$ (dyn, m.) 


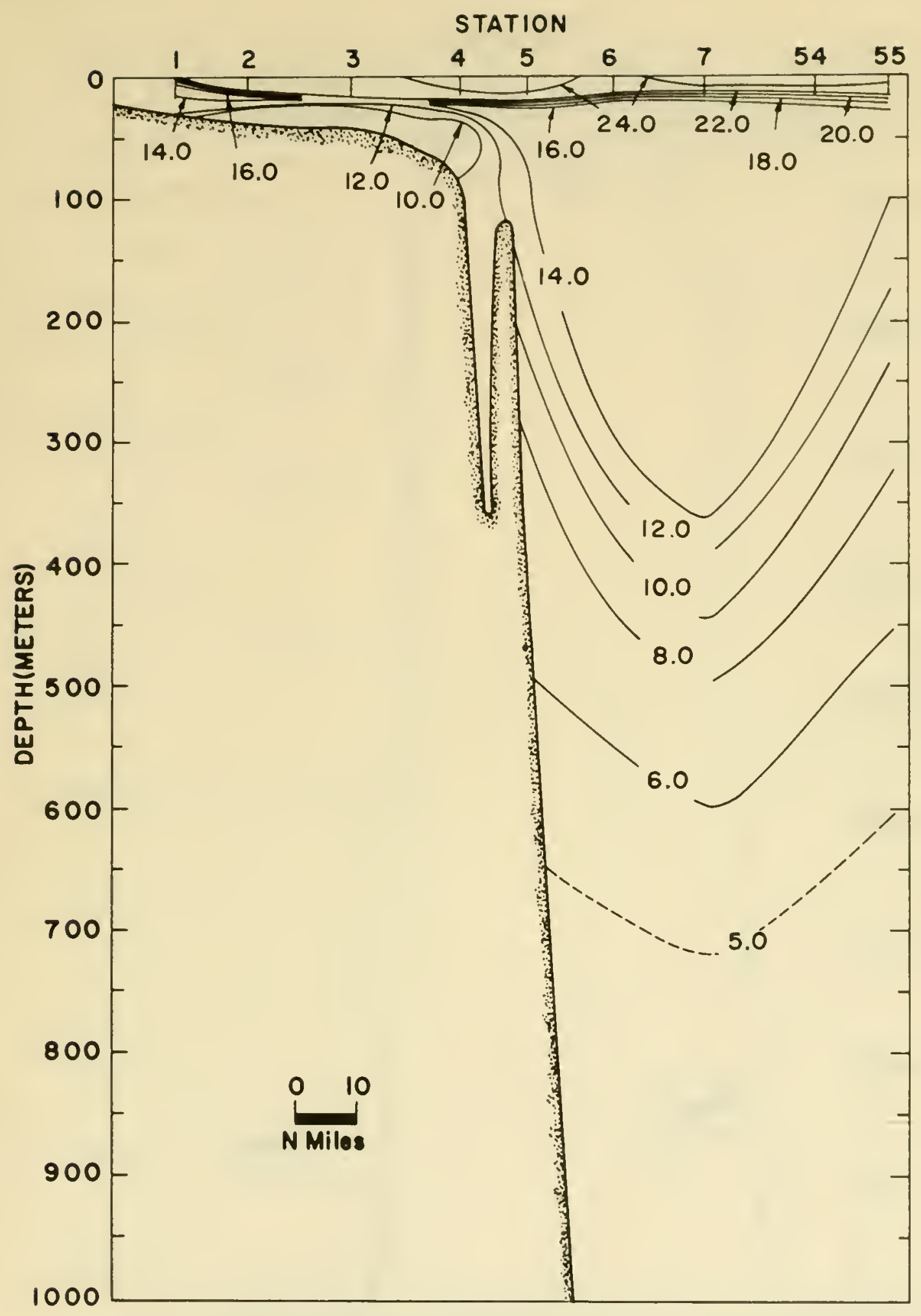

Figure 7.-Vertical distribution of temperature, section A, August $1974\left({ }^{\circ} \mathrm{C}\right)$ 


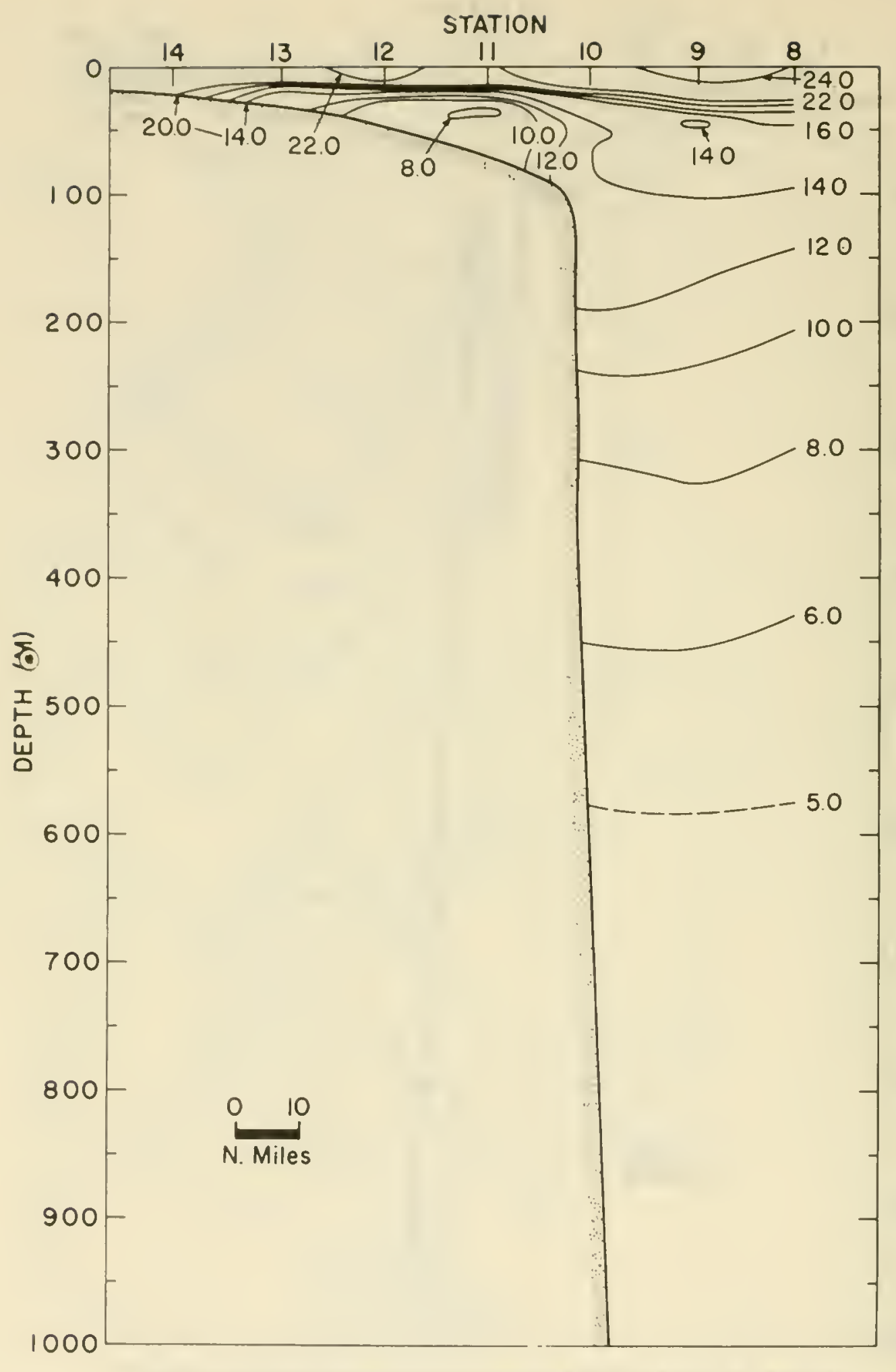

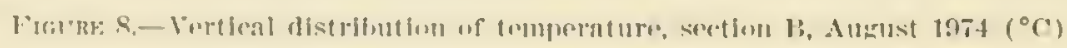




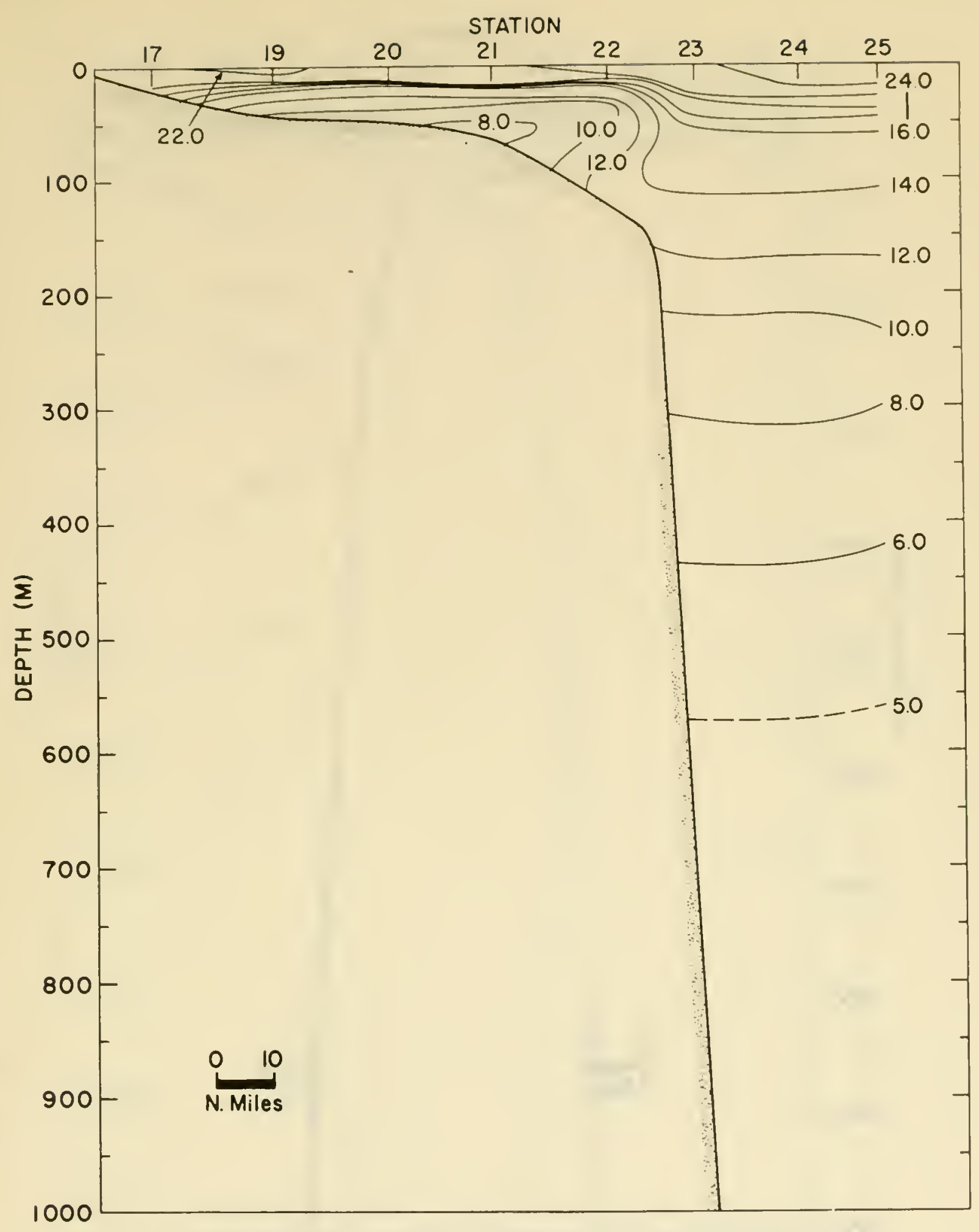

Fictere 9.-Tertical distribution of temperature, section C, August $1974\left({ }^{\circ} \mathrm{C}\right)$ 


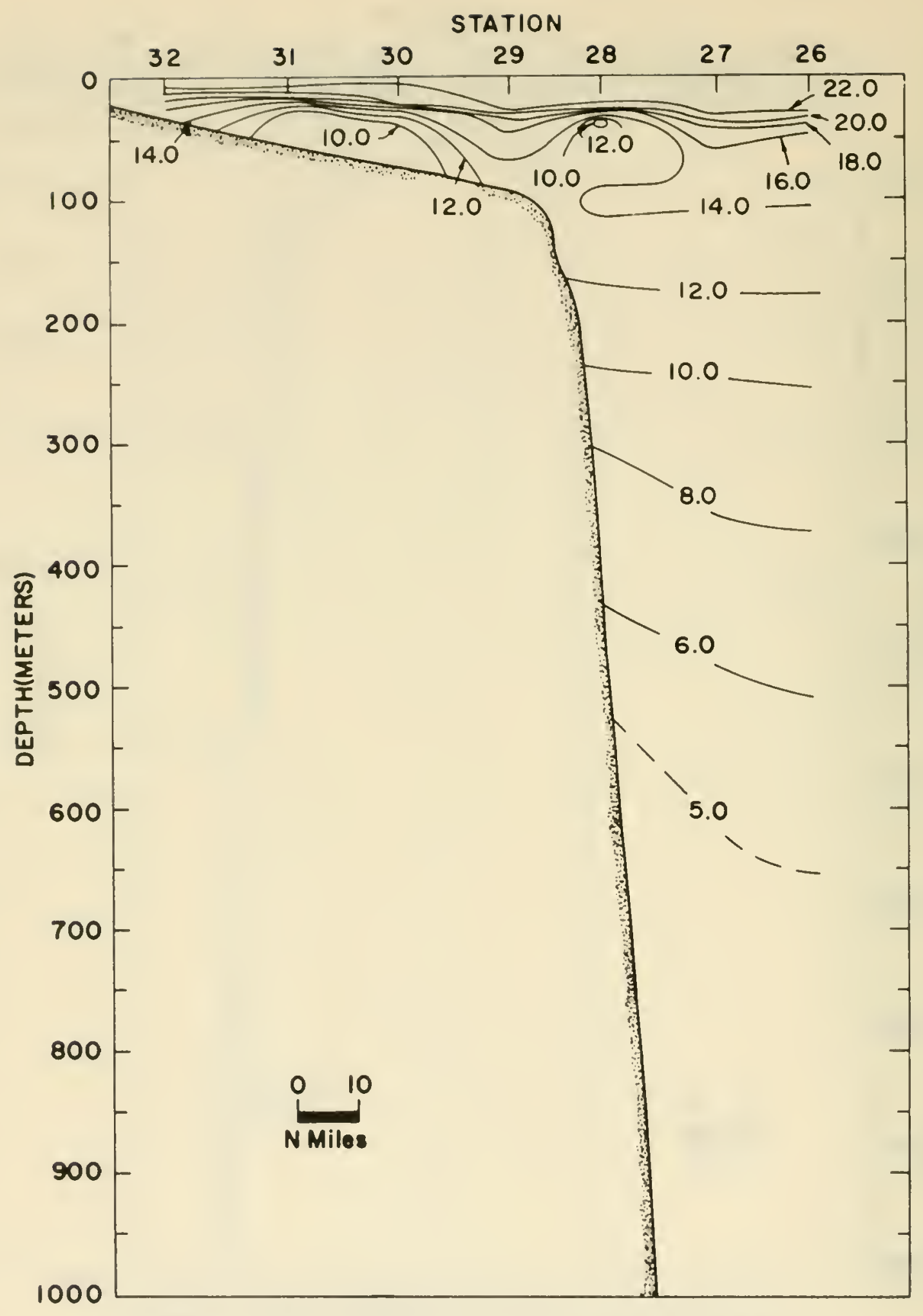

Frovir: 10. - Vertical distrihution of temperature, section $\mathrm{D}$, August $1974\left({ }^{\circ} \mathrm{C}\right)$ 


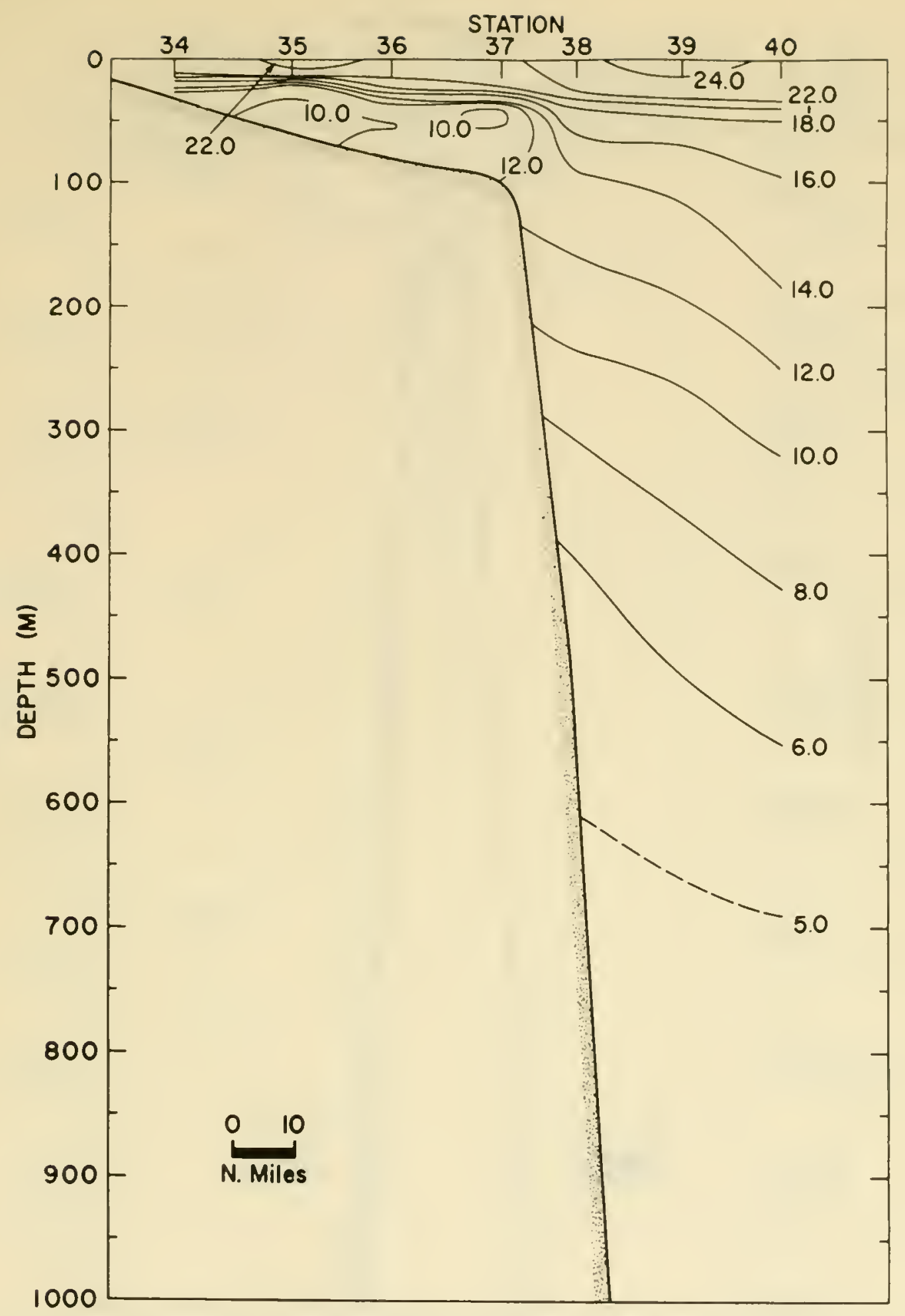

Figtre 11. - Vertleal distribution of temperature, section E, August $1974\left({ }^{\circ} \mathrm{C}\right)$ 


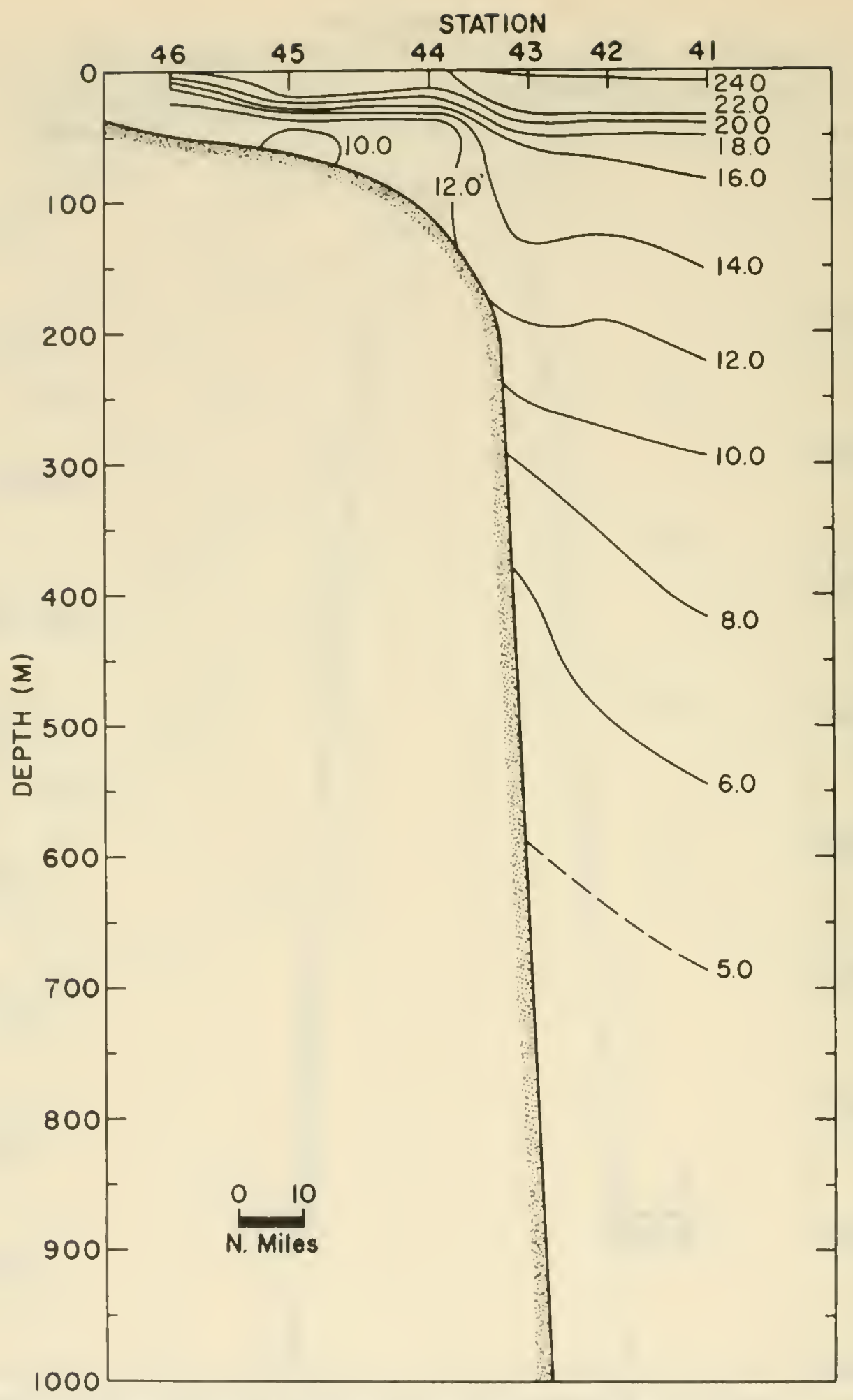

Figcen: 12-lertical distribution of temperature, seretion $\mathrm{F}$, August $1974\left({ }^{\circ} \mathrm{C}\right)$ 


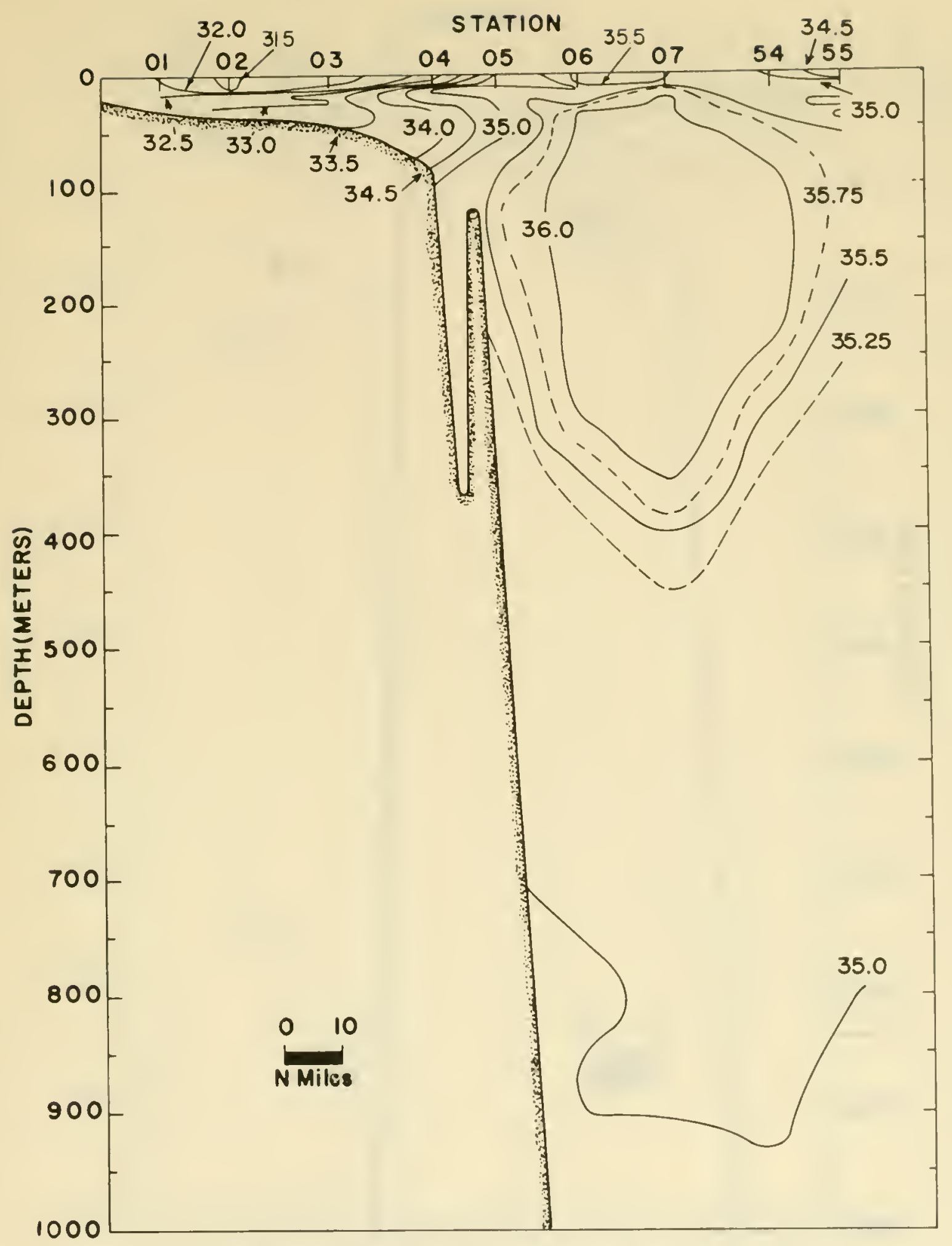

Figtore 13. Vertical distribution of salinity, section $\Lambda$, August $197+(\%)$ 


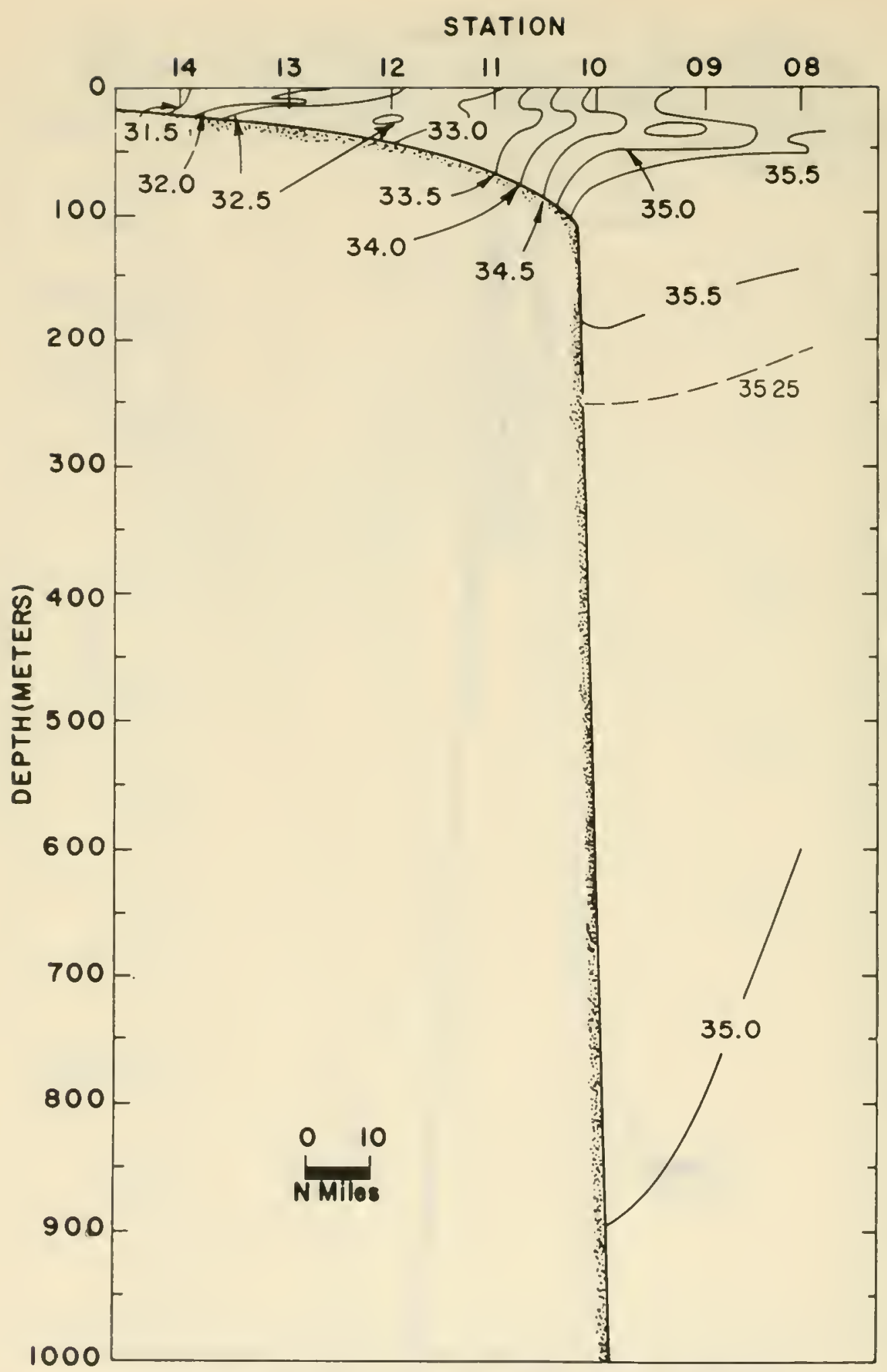

Figure 14.-Vertical distribution of salinlty, sectlon B, August $1974(\%)$ 
STATION

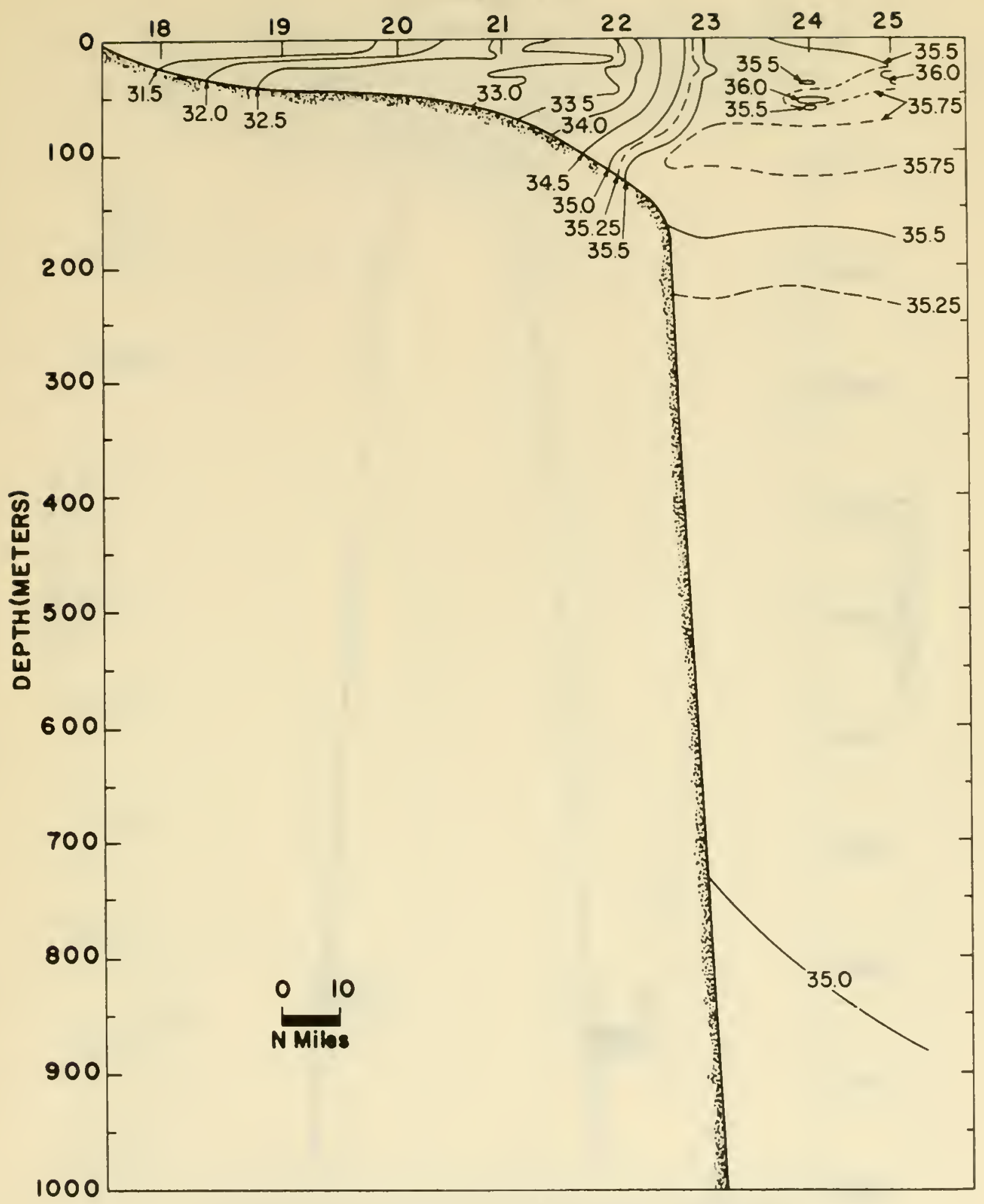

Figure, 15.-Vertical distriluution of salinlty, section C, August $1974(\%)$ 


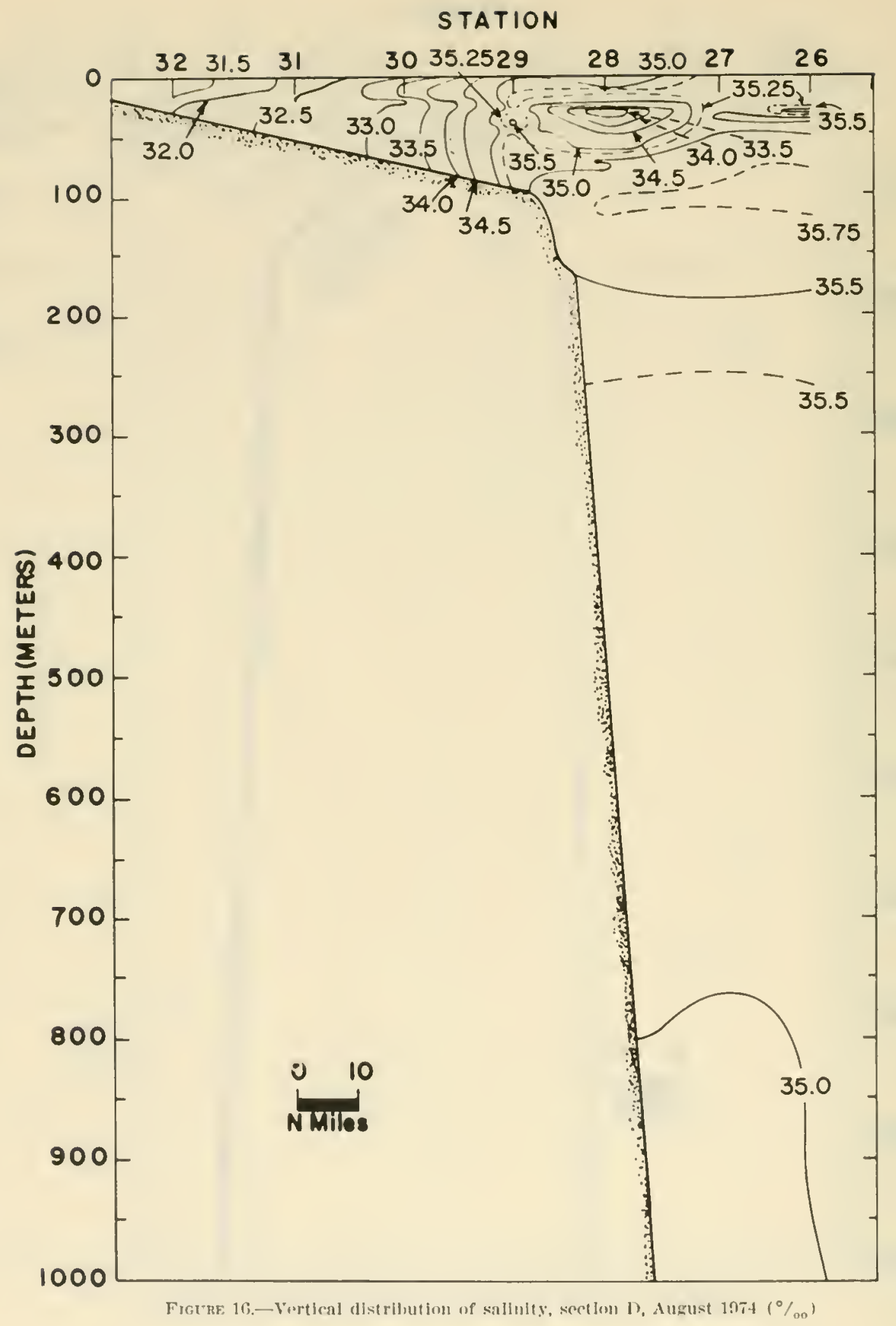




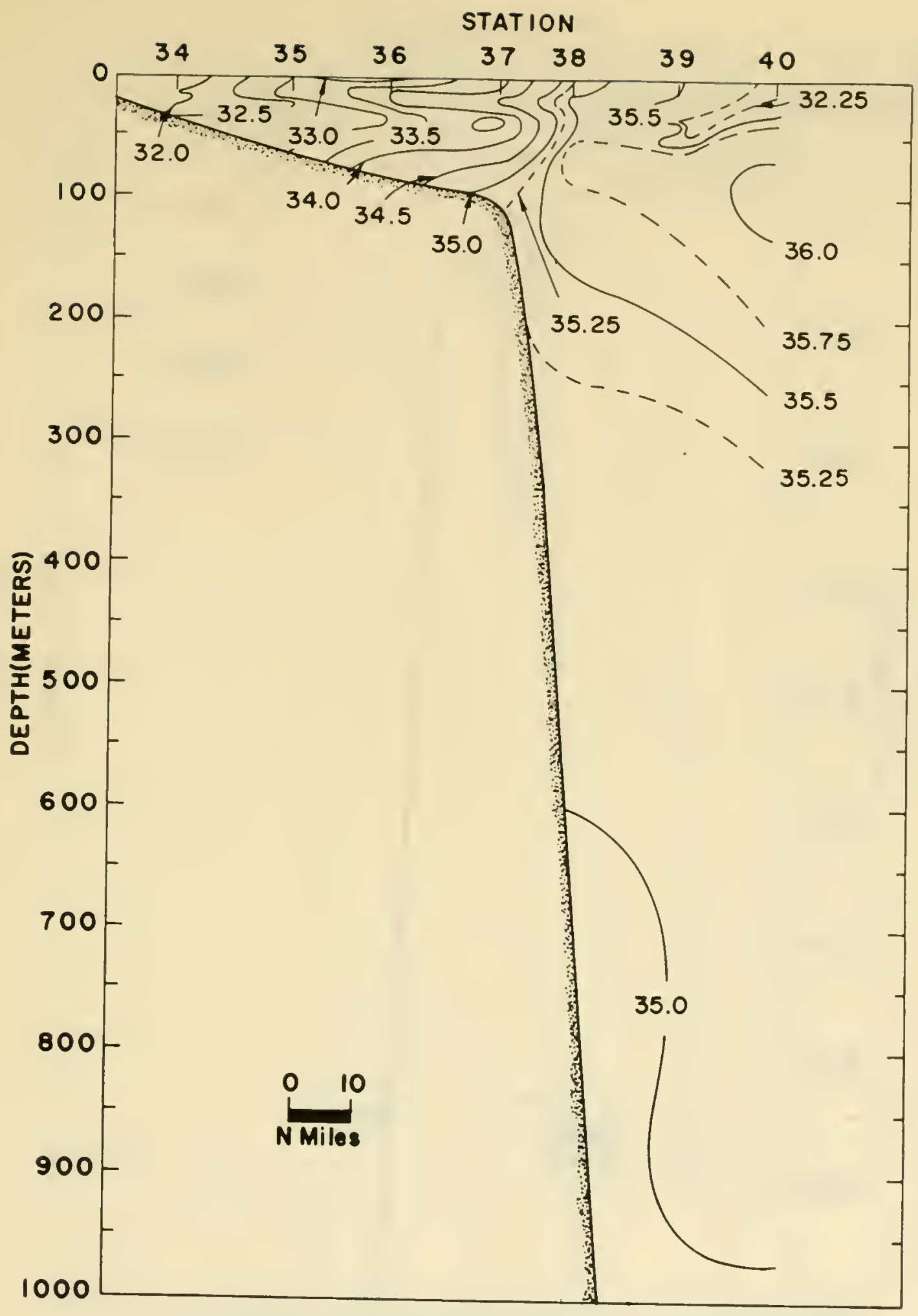

Figune 17 . - Vertical distribution of salinity, section E, August $1974(\%)$ 
STATION

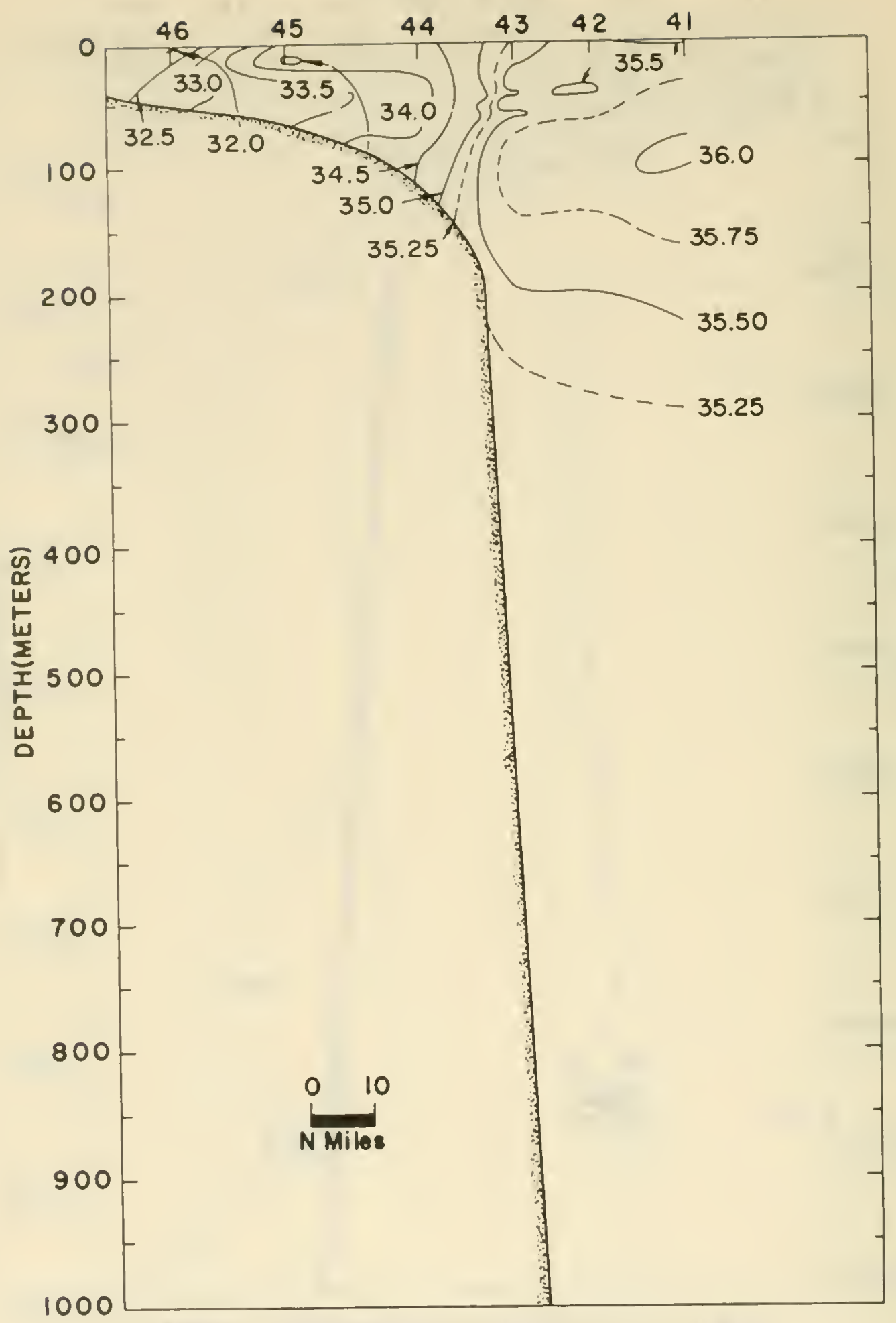

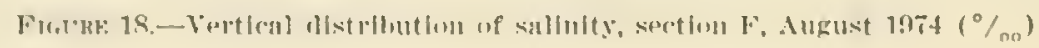




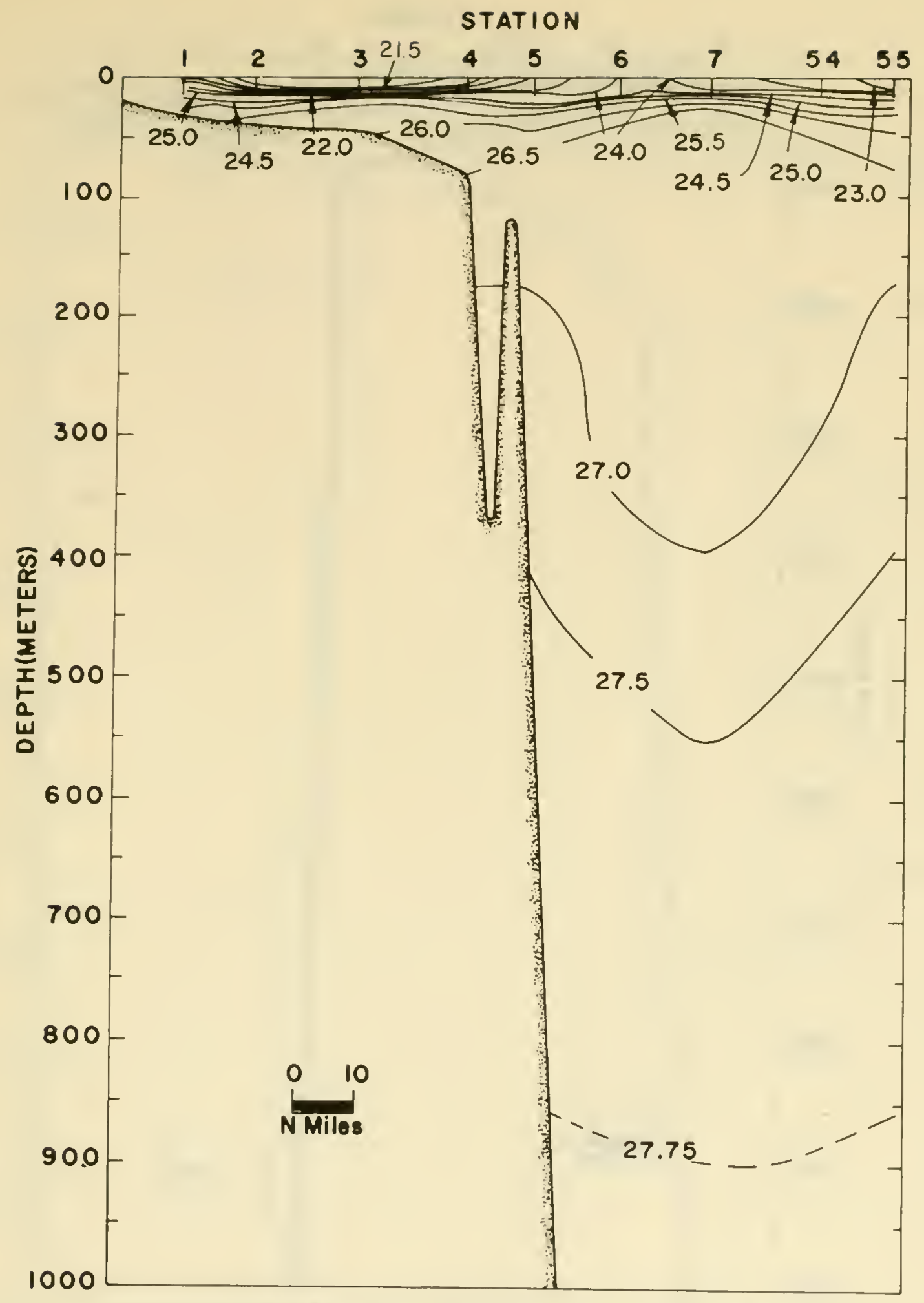

Figres: 19.-Fertical distrihutum of sigma-t, spetion A, August 197t 


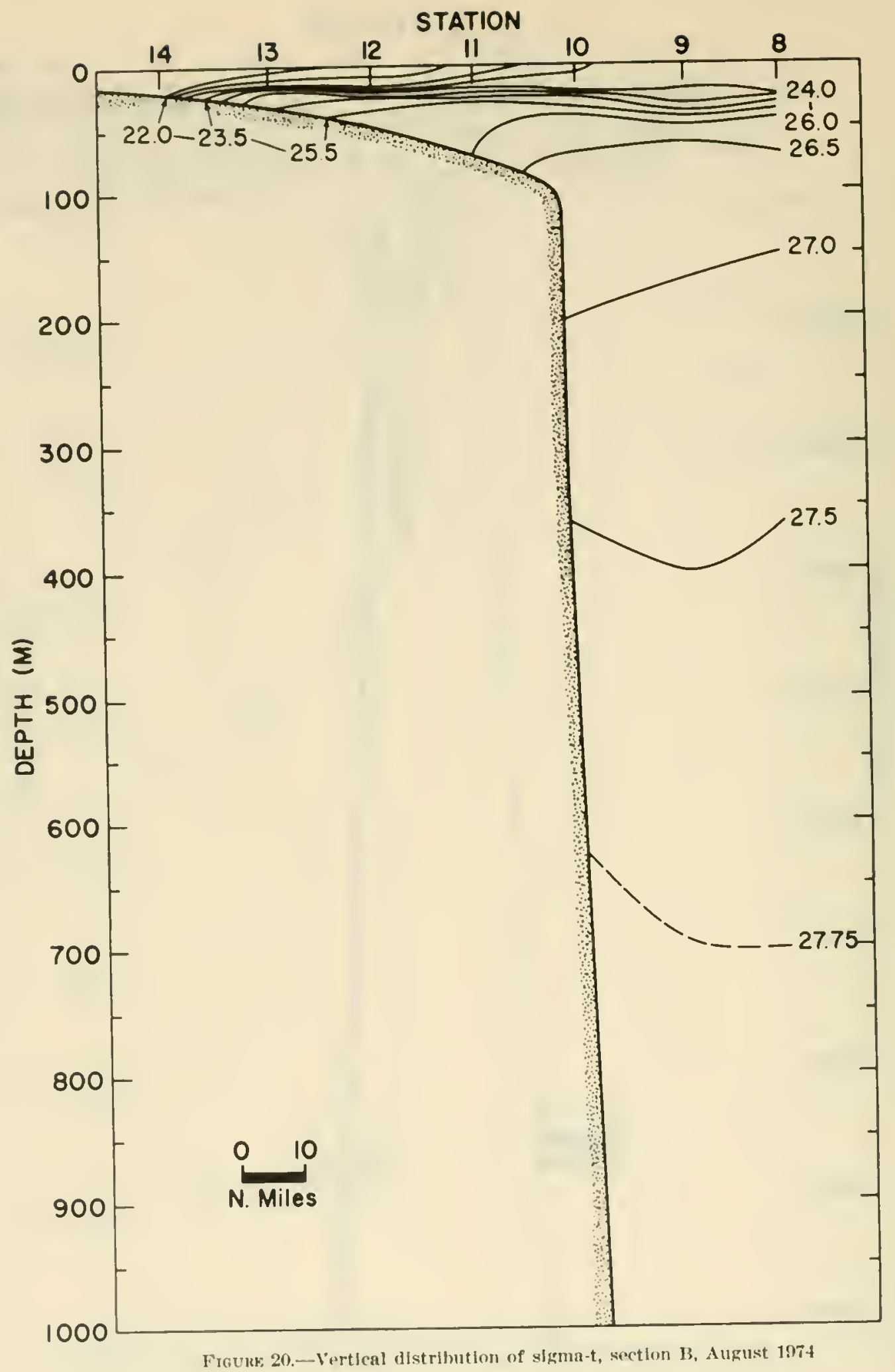




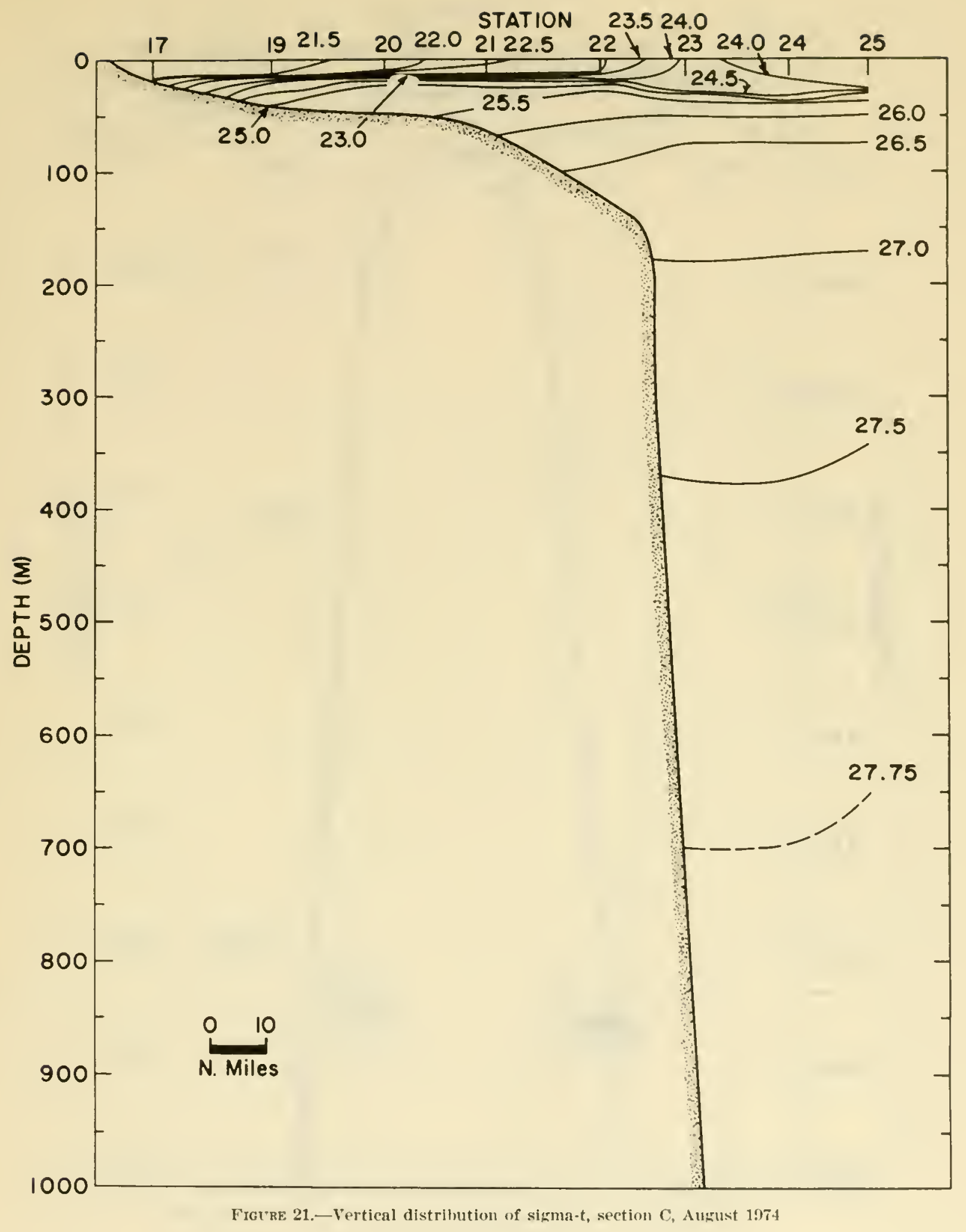




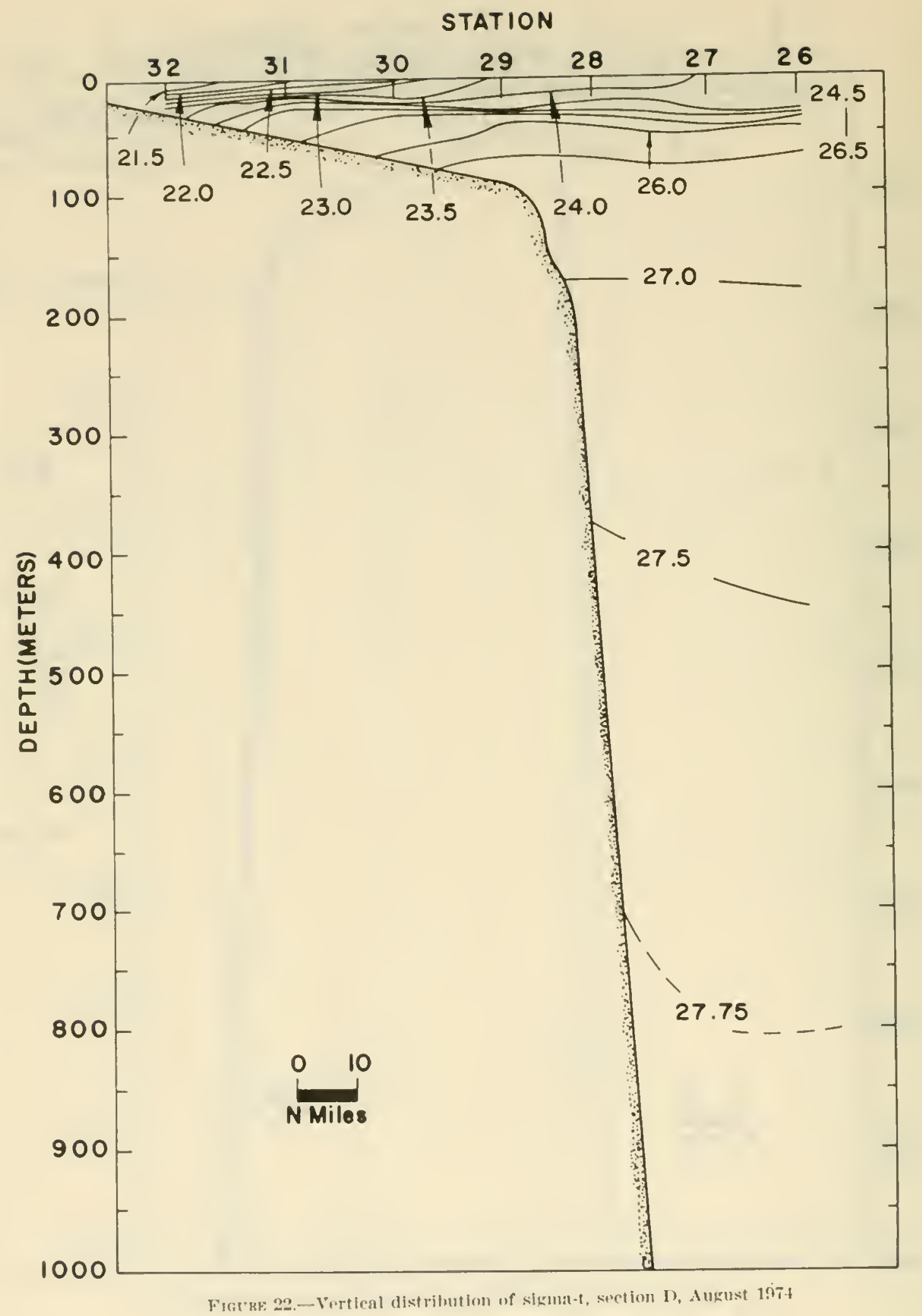




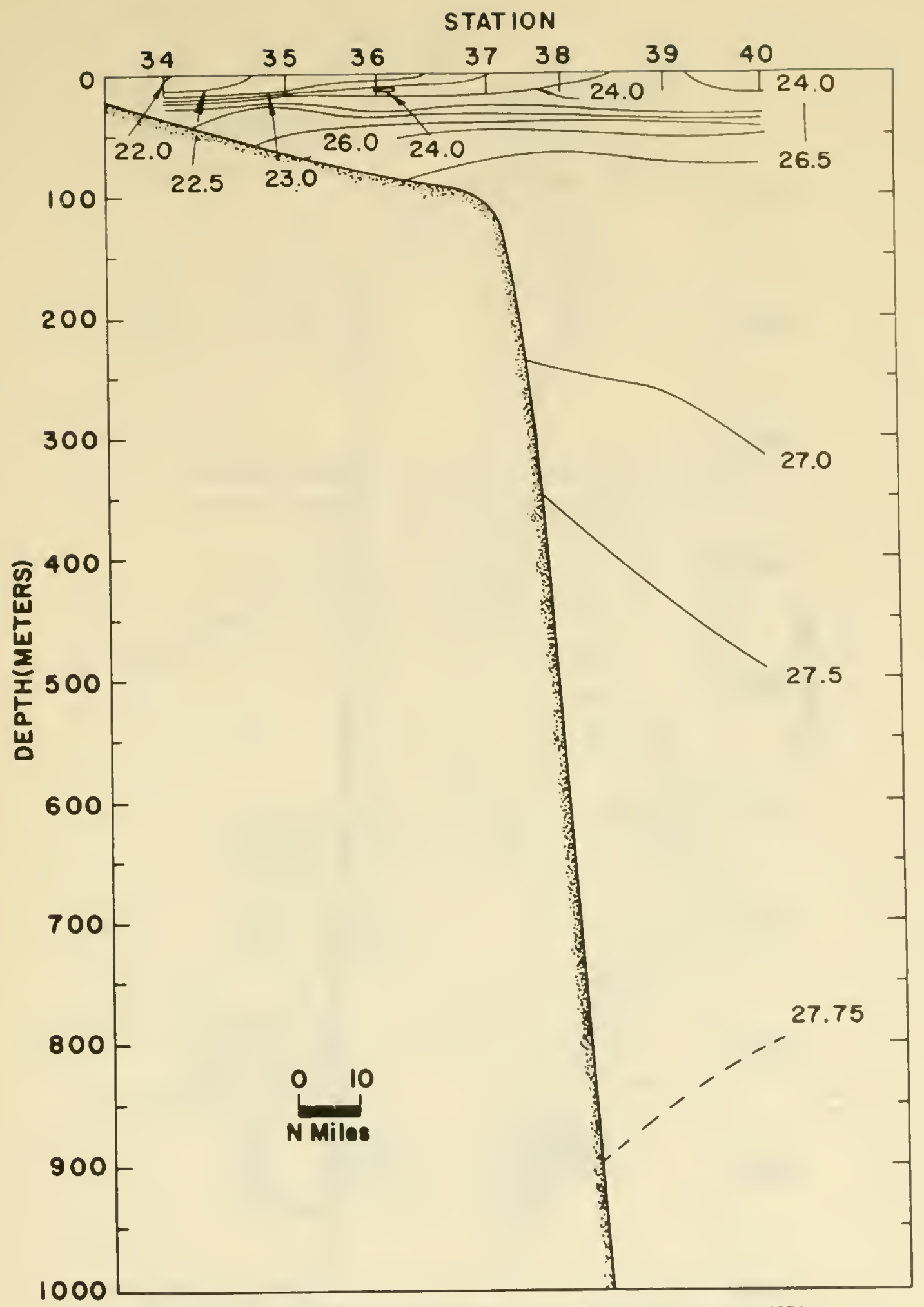

Figtre 23. -Vortical aistribution of sigma-t, section $\mathrm{F}$, August 1374 


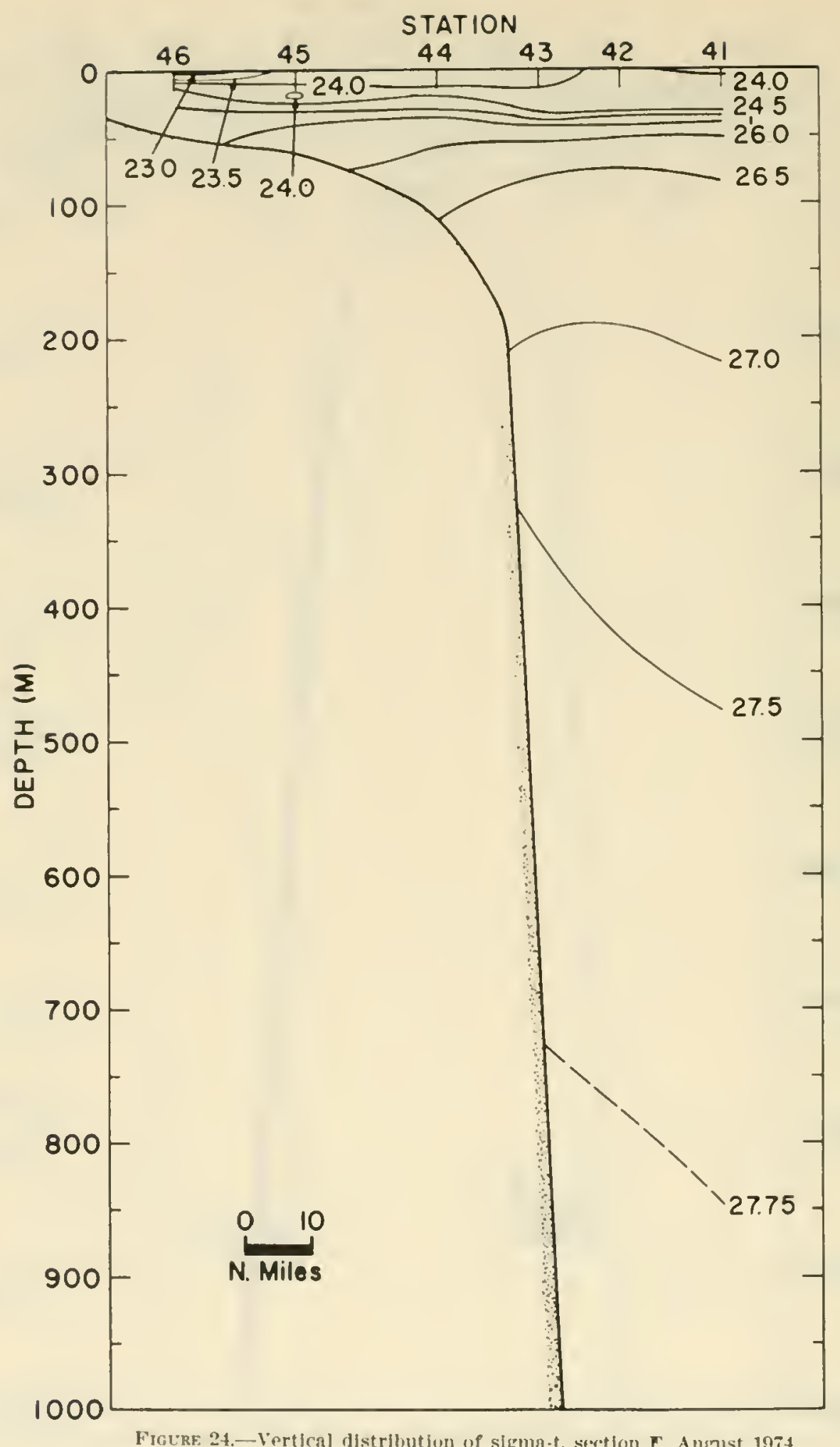




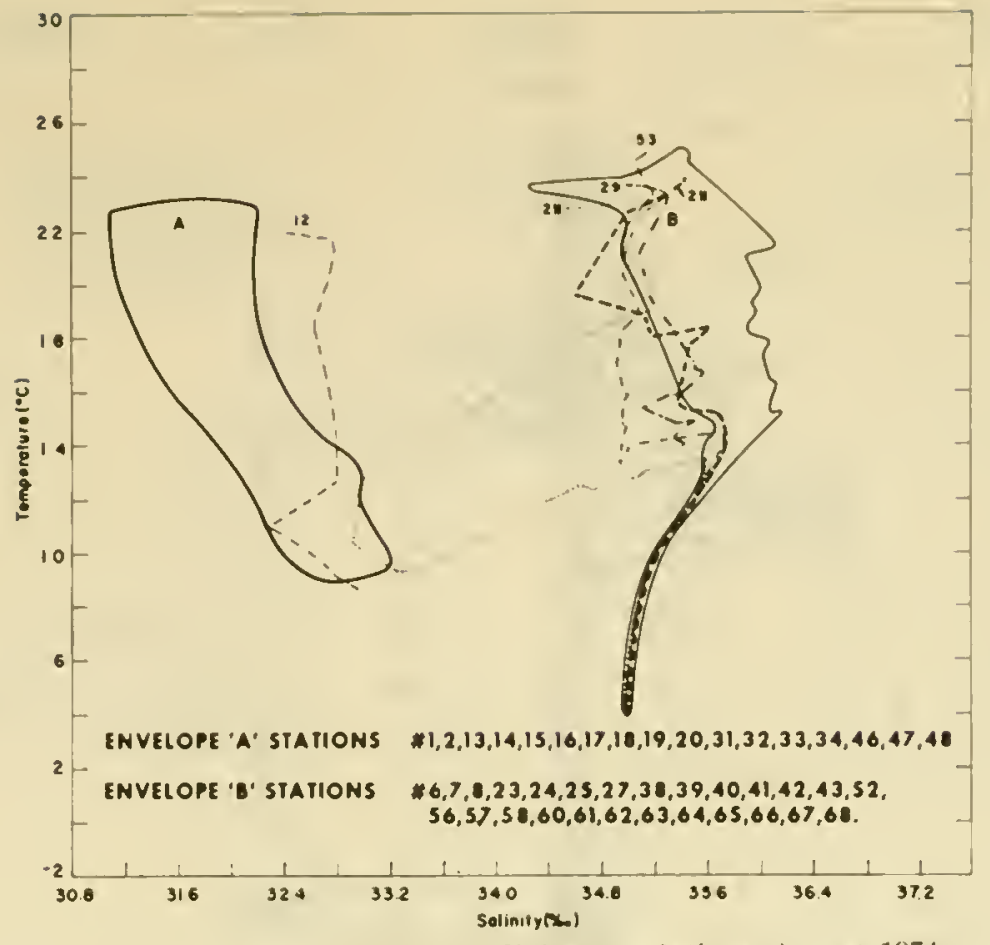

Figure 25a.-Temperature-salinity correlations, August 1974

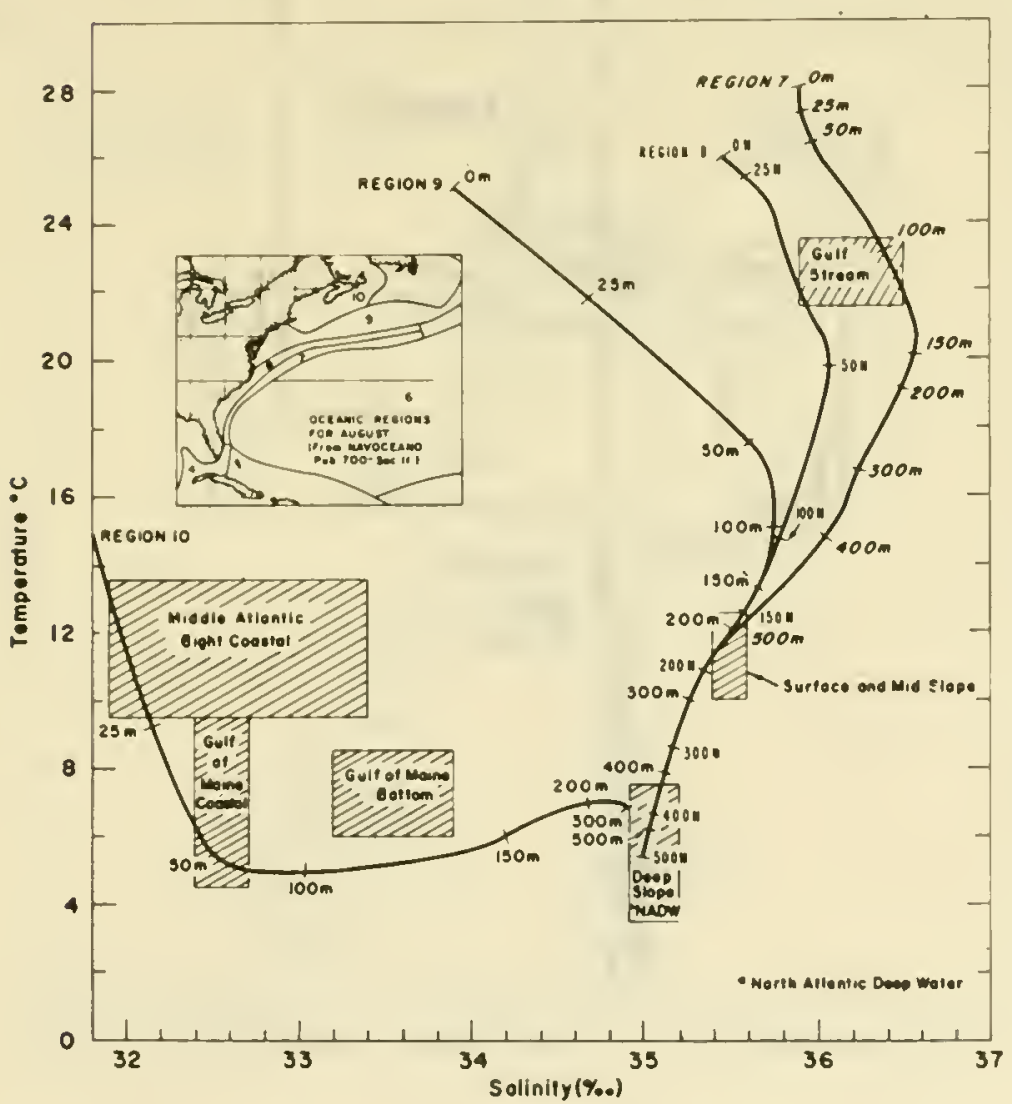

Figrer: 25h,-August temperature-salinity curves from NoO l'uh 700 , Sec. II, and combined spring and fail water mass ranges from Hayes $(1975)$ 


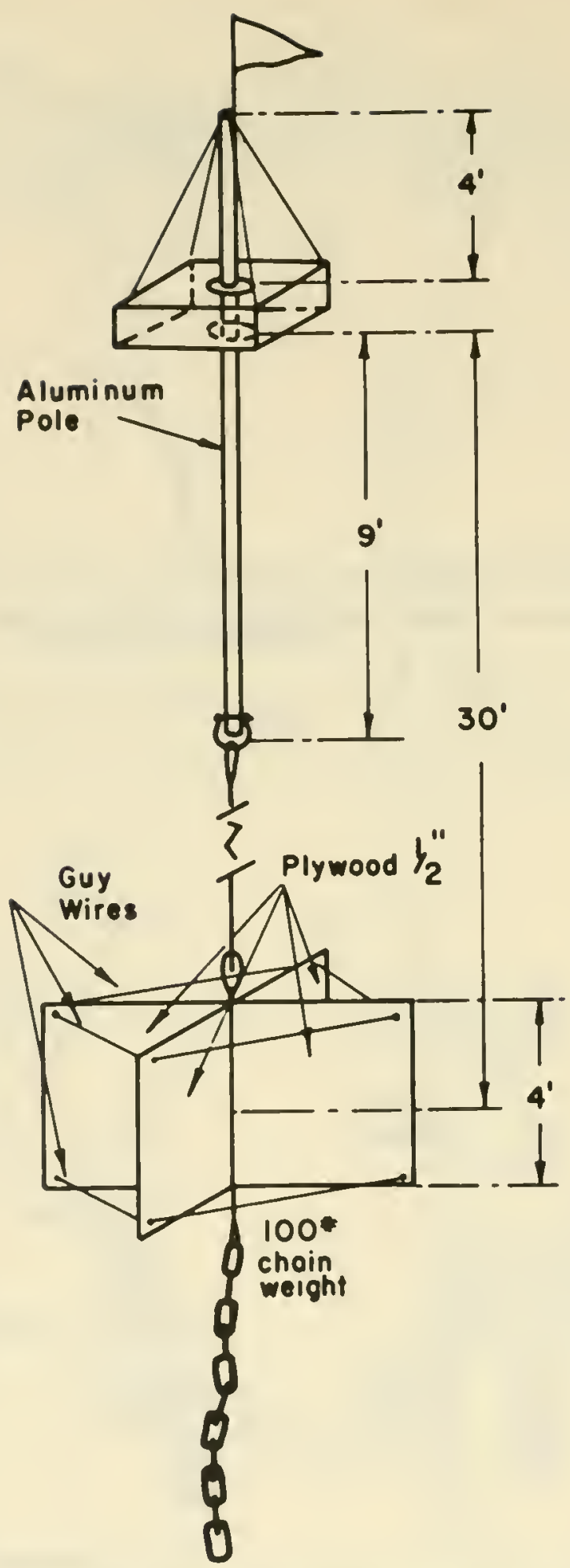

Ficuke 26.-Surface (30 fout depth) current drogue 


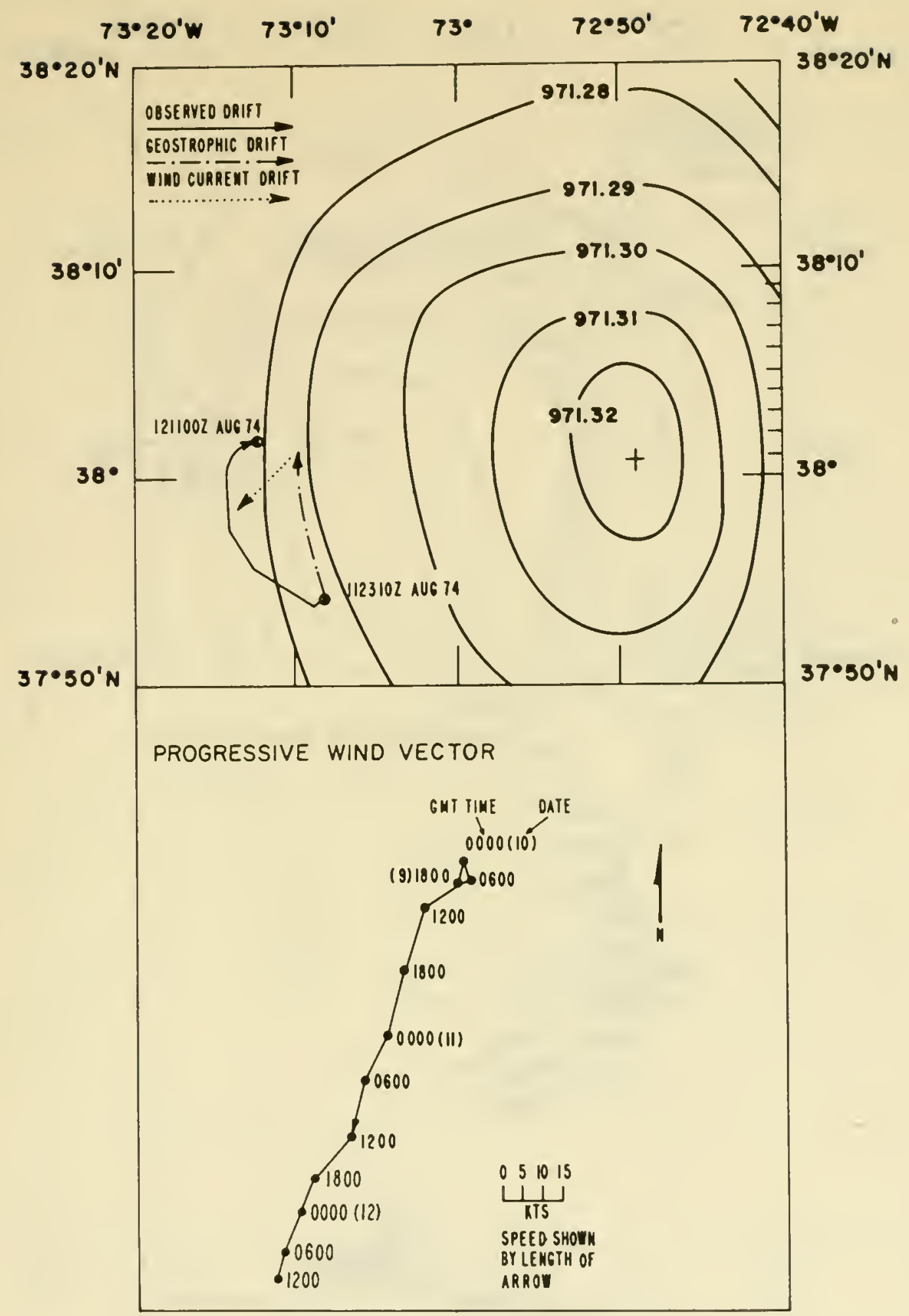

Figeke 27.-Drogue movement $2310 \% 11$ August to $1100 \% 12$ August, 1974 


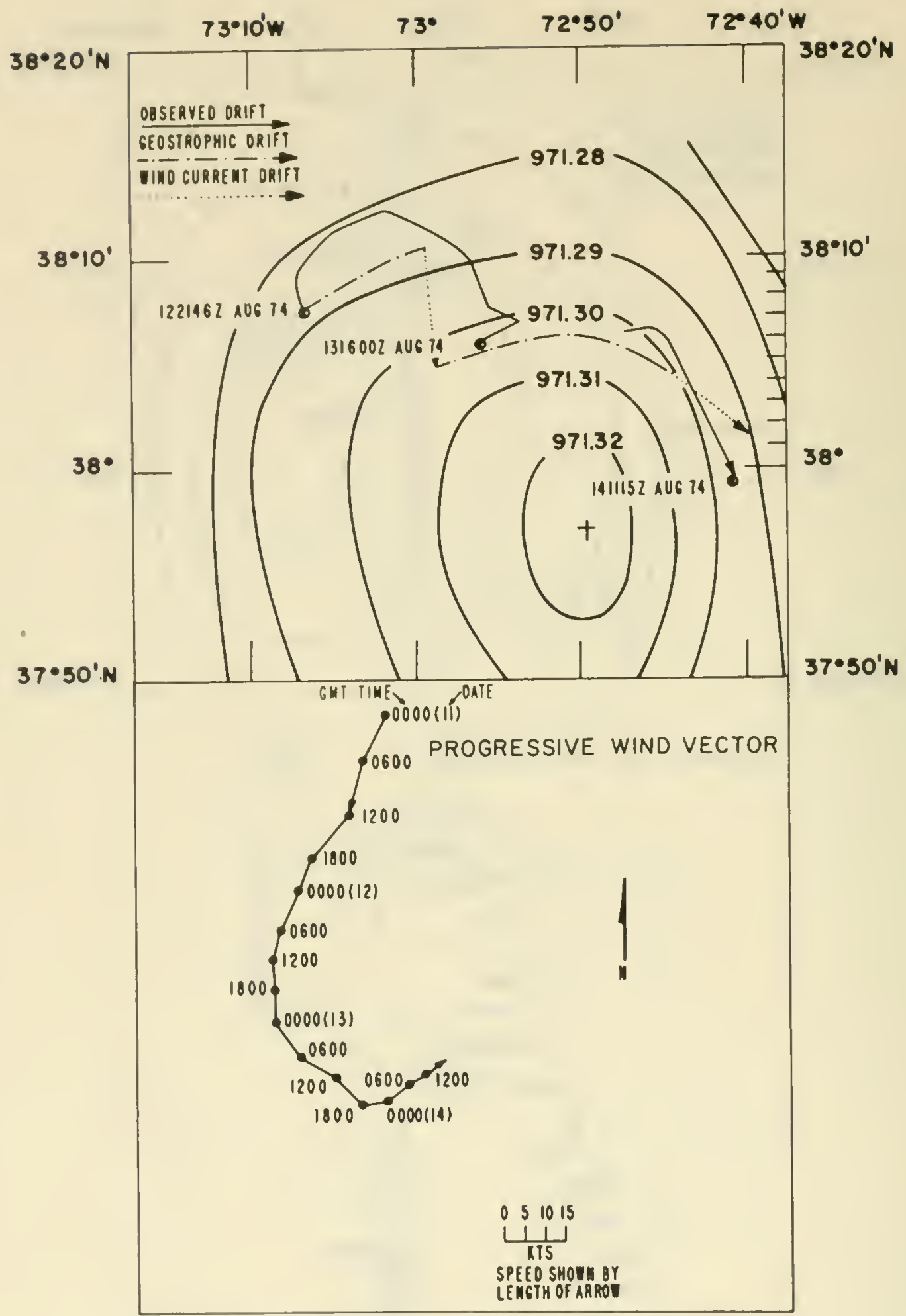

Figur: 28.-I Trogur movement 2146Z 12 August to 111571.4 August 1974 


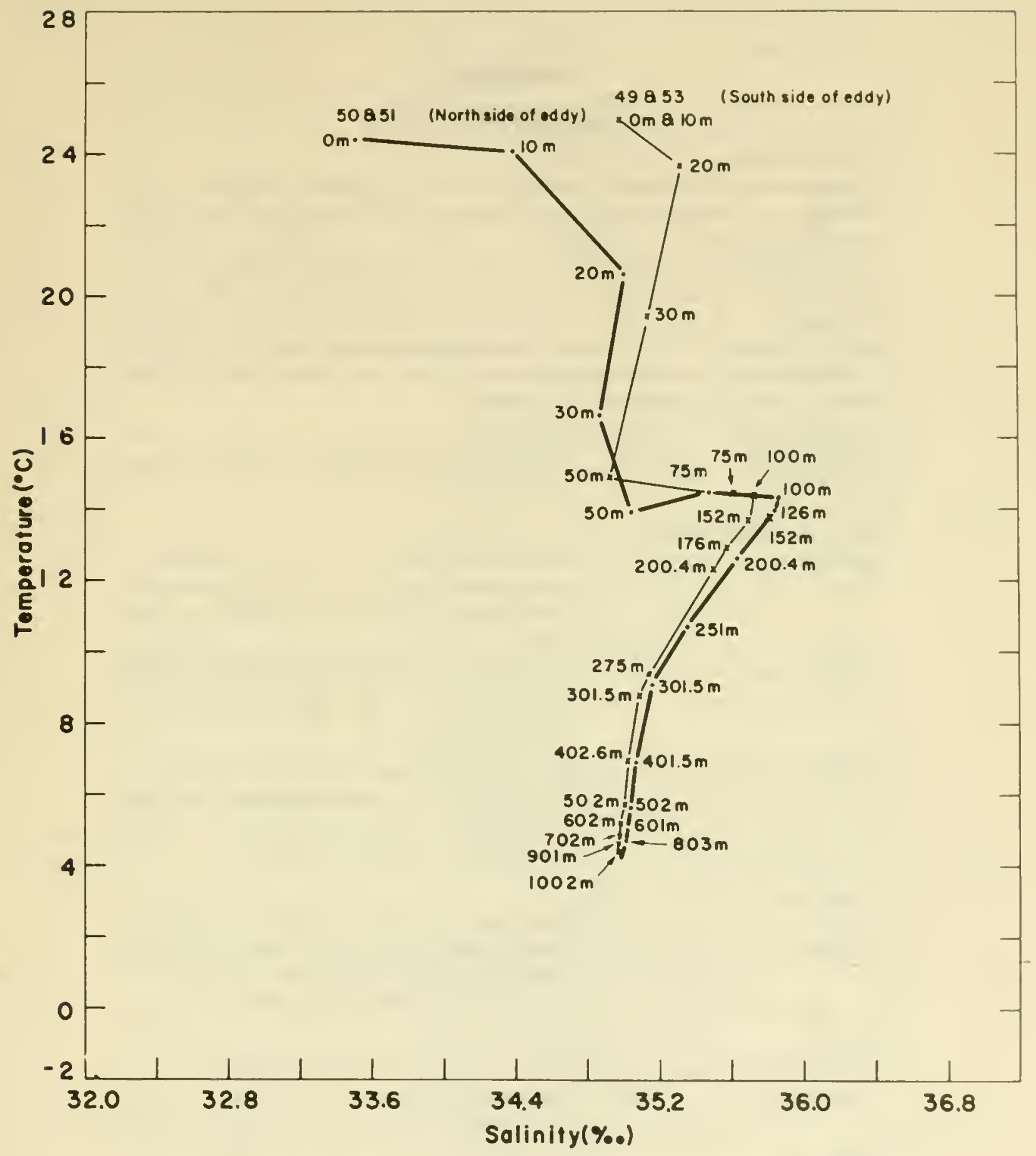

Figure 29.-A verage temperature-sallnity correlations, statlons, 49, 50, 51, ant 53 


\section{APPENDIX A \\ OCEANOGRAPHIC DATA}

\section{Cruises Listed}

Observed and interpolated oceanographic data taken by USCGC EVERGREEX, 8-20 August 1974 on Ni̊R Cruise $3-i t$, prepared from NODC Listing No. 318408.

$A$ complete description of the codes utilized in the tabulation of oceanoEraphic station data can be found in National Oceanographic Data Center publication M-2, Processing Physical and Chemical Data from Oceanographic Stations. (Rev. August 196t, supplement issued May 1966.)

To facilitate use of the oceanographic station data listing, entry headings which are not self-explanatory are described below.

Depth to Bottom _.........._Corrected ol uncorrected sounding in meters. Max. Depth of Samples _._-_-_Depth of deepest sample to nearest multiple of one hundred meters.

\section{Wave observations}

DIR. _..._._._._._. Rounded to nearest multiple of 10 degrees.

HGT. In increments of $1 / 2 \mathrm{~m}$. Sum of 5 meters plus increments of $1 / 2 \mathrm{~m}$ if 50 is added to direction.

PER.

If numerals 2 through 9 are entered, period in seconds is twice the numeric entry of $2 \mathrm{X}$ (numeric entry) +1 . For other entries see IIMO Code 3155.

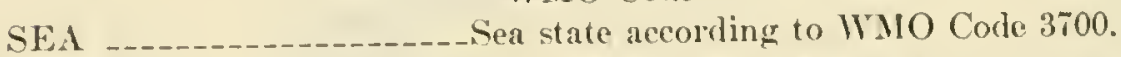

Weather Code _._._._-___._. If preceded by $\mathbf{X}$, weather according to WMO Code 4501. If a two-digit entry, weather according to WMO Code $46 \pi i$.

Cloud Code

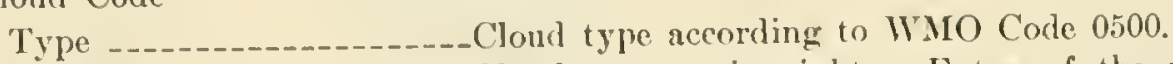

Amount Cloud amount in eights. Entry of the numeral 9 indicates cloud amount could not be estimaterl. (WMO Code 2700 )

Water

Color Code _._._._._-_._Color accorling to Forel-Ule scale.

'Truns. Transparency in whole meters as determined by Seechi disc.

Wind

Dir. Rounded to nearest multiple of 10 degrees.

speed or Force If preceded by letter $S$, wind speed in knots; if preceded by letter $F$, wind force according to Beaufort scale. 

tenths of millibars.

Air Temp. ${ }^{\circ} \mathrm{C}$ grade.

Vis. Code

Visibility according to WMO Code 4300.

No. obs. depths

Number of observed levels associated with the station.

Messenger time

Entered in hours and tentlis of an hour GMT. For Nansen casts, indicates time of release of messenger applicable to the observational level. For STD casts, indicates the starting time of lowering the sensor.

Card type _.................. cates the values at this standard level were interpolated by a modified 3-point LaGrange formula.

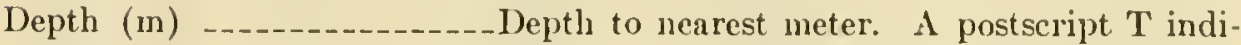
cates depth was obtained thermometrically; $\mathrm{Z}$ indicates uncorrected "wire out" depth. Postscript $Q$ indicates value was marked doubtful by originator; $\mathrm{P}$ indicates value was considered doubtful by NODC. Postscripts $P$ and $Q$ retain this meaning throughout the following entries.

$\mathrm{T}^{\circ} \mathrm{C}$

Temperature to hundredths of a degree Centigrade.

$\mathrm{S} \%$

Salinity in parts-per-thousand.

SIGMA-T

Entered to hundredths.

Specific-volume

Multiply entry by $10^{-7}$ to obtain specificvolume anomaly in cubic centimeters per gram.

$\Sigma \Delta$ D Dyn. M. $\times 10^{3}$

Multiply entry by $10^{-3}$ to obtain anomaly of dynamic height in dynamic meters referenced to the sea surface.

Sound Velocity _............-Sound velocity according to Wilson's formula entered to tentlis of a meter per second.

$\mathrm{O}_{2} \mathrm{ml} / 1$

Dissolved oxygen in milliliters per liter entered to hundredths.

$\mathrm{PO}_{4}-\mathrm{P}$ ug-at/1 _............. Inorganic phospluate in microgram-atoms per liter entered to hundredths.

Total-P ug-at/1 _........-...-Total plosphorus in microgram-atoms per. liter entered to hundredths.

$\mathrm{NO}_{2}-\mathrm{N}$ ug-at/1 _.............. Nitrite-nitrogen in nicrogram-atoms per liter entered to hundredths.

$\mathrm{NO}_{3}-\mathrm{N}$ ug-at/1 _............ Nitrite-nitrogen in microgram-atoms per liter entered to tenths.

$\mathrm{SiO}_{4}-\mathrm{Si}$ ug-at $/ 1$

Silicate-silicon in microgram-atoms per liter entered to whole units.

CHL-A Chlorophyll-A (total pigment) in milligrams per cubic meter entered to hundredths. 
$\begin{array}{lrr}\text { REFIO } & 31 & 8408 \\ \text { CCNSEC } & 0001 \\ \text { LAT } & 38 & 56.14 \\ \text { LJNG O74 } & 26.3 \%\end{array}$

VEAR 1974

MONTH OE

OAY OB

MOUR 19.8 80top 00029

SNIP EV

OATH USE I

AL IEAP 23.1 MET BULB 20.3

BAKOMEIR 1022.9

CluUO I/A

OIR HGI PER
20 O 2
SEA
CIJIA

-IVO-DIR 20

WIVO-SPO OU

IINO-FOR
IMST SIJ AECOROER TEM SO 1200 THACE OIR OLOS SOUAAE 3 ORIG 37600113 I SOUARE

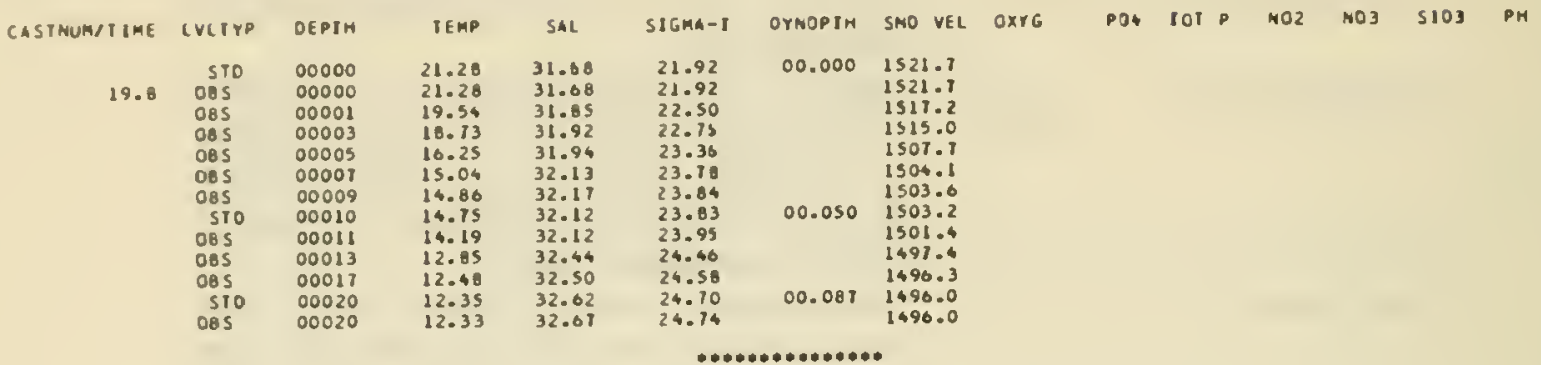

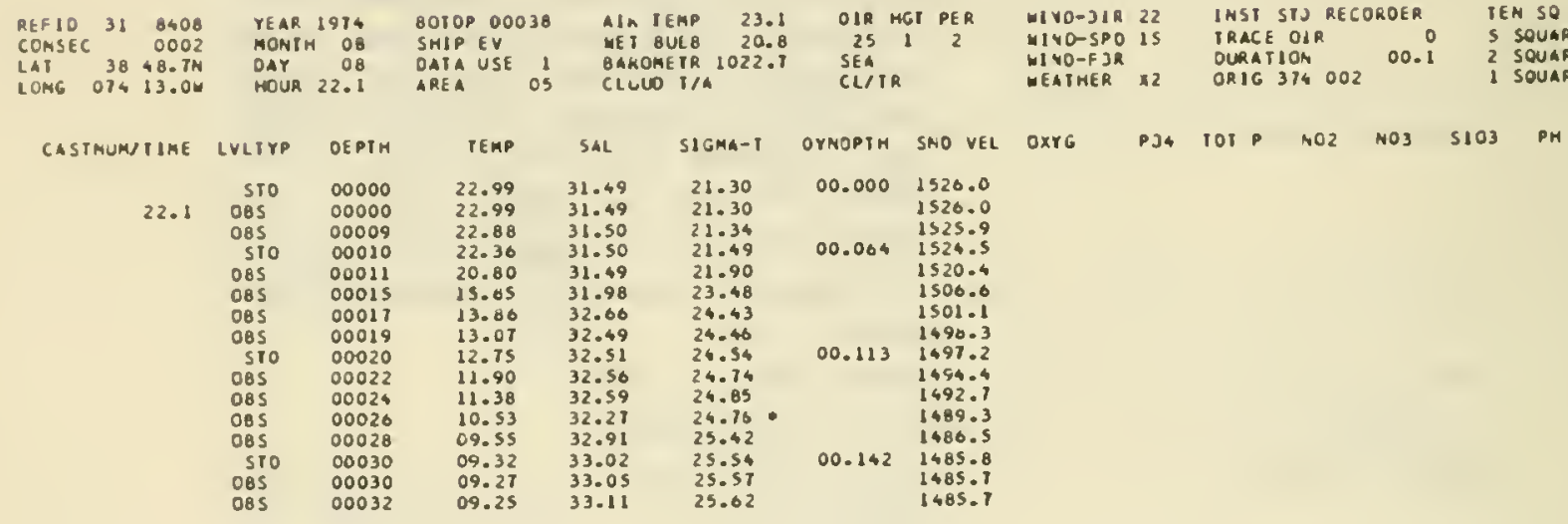

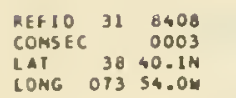

YEAR 1974 MONTH 08 Mar 09
BOTOP 00042 SHIP EV

ORTA USE I AIN IEMP
WET BULB 24.6
BAKOMETR 1022.5 CLLUD T/A
OIR HGI PER

SEA

CLA
MI VO-JIR 21

WIMO-SPO 12

WIMO-FOR

WEIMER X2
INST STU RECOROER TEN SO 1209 TRACE OIP. S SQUARE 3 OURATION OO.1 2 SOUARE 82

$\begin{array}{ccccc}\text { TEMP } & \text { SAL } & \text { SIGMA-T } & \text { OYNOPIM } & \text { SHO VEL } \\ 23.35 & 31.62 & 21.30 & 00.000 & 1527.0 \\ 23.35 & 31.62 & 21.30 & & 1527.0 \\ 23.31 & 31.00 & 21.30 & 00.065 & 1527.1 \\ 23.31 & 31.60 & 21.30 & & 1527.1 \\ 20.81 & 32.40 & 22.59 & & 1521.5 \\ 16.41 & 33.25 & 24.32 & & 1510.1 \\ 14.09 & 33.32 & 24.89 & 00.113 & 1502.7 \\ 12.32 & 33.33 & 25.25 & & 1496.8 \\ 08.66 & 32.86 & 25.52 & & 1483.1 \\ 08.34 & 33.15 & 25.79 & & 1482.3 \\ 08.19 & 33.23 & 25.88 & 00.139 & 1481.8 \\ 08.15 & 33.25 & 25.90 & & 1481.7 \\ 08.09 & 33.28 & 25.93 & & 1481.6 \\ 08.09 & 33.26 & 25.92 & & 1481.7\end{array}$

OXYG PJ4 TOT NOZ NO3 SIO3 PM

$\begin{array}{ccc}\text { STO } & 00000 \\ \text { OBS } & 00000 \\ \text { STO } & 00010 \\ 085 & 00011 \\ 085 & 00013 \\ \text { OBS } & 00019 \\ \text { S10 } & 00020 \\ \text { OBS } & 00020 \\ \text { OAS } & 00024 \\ \text { OES } & 00028 \\ \text { STO } & 00030 \\ \text { OBS } & 00030 \\ \text { OBS } & 00034 \\ \text { OES } & 00040\end{array}$

$0.09 \quad 33.26$

25.92 


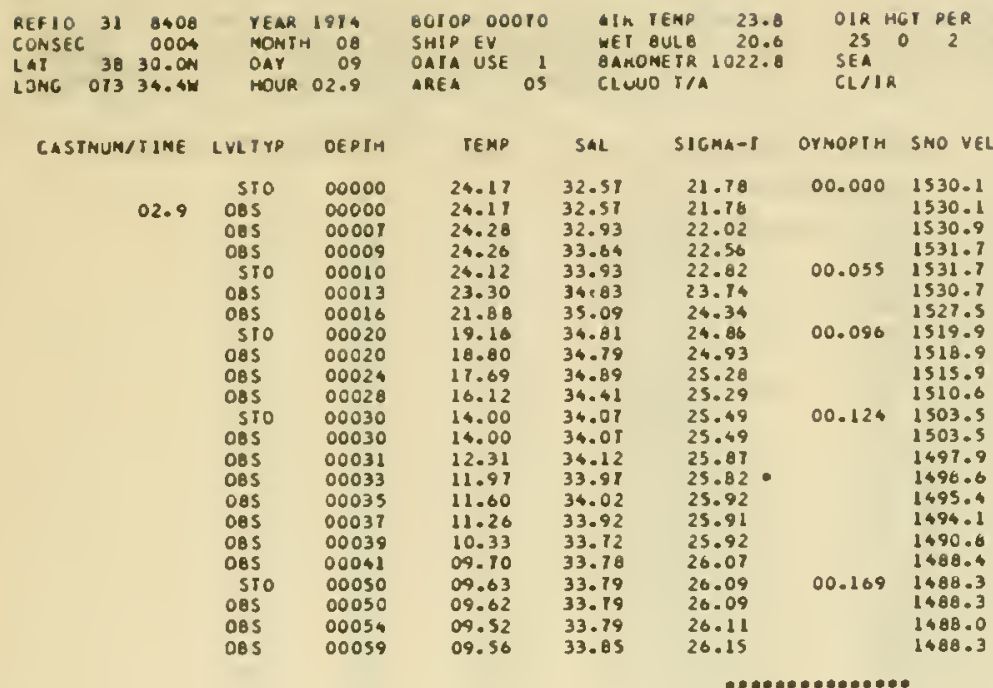

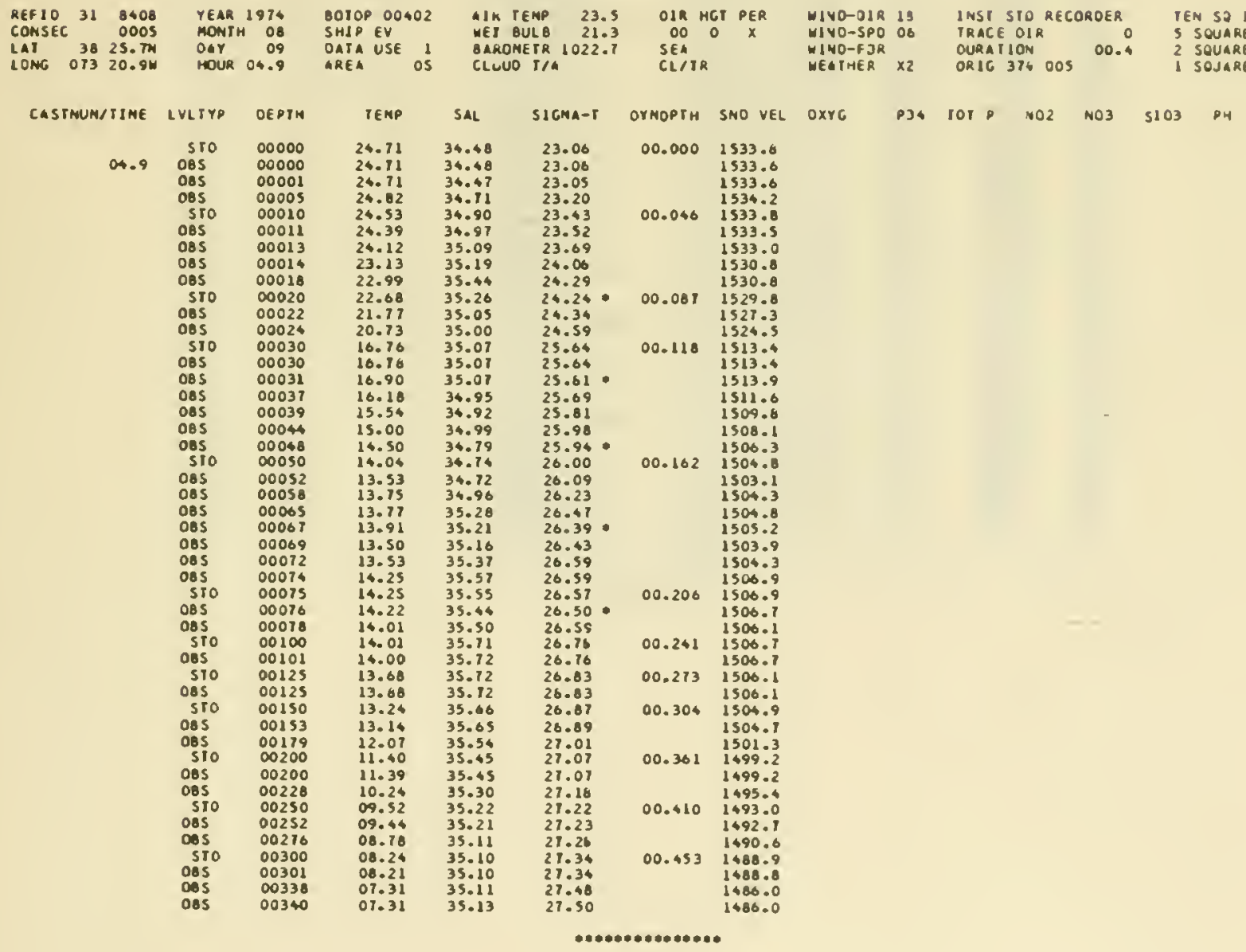




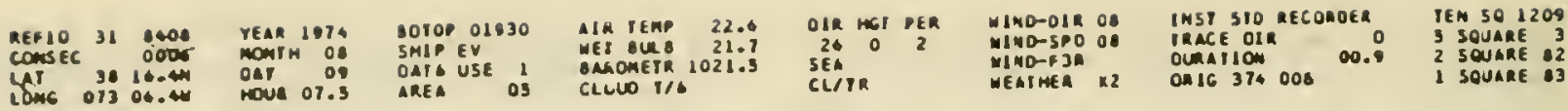

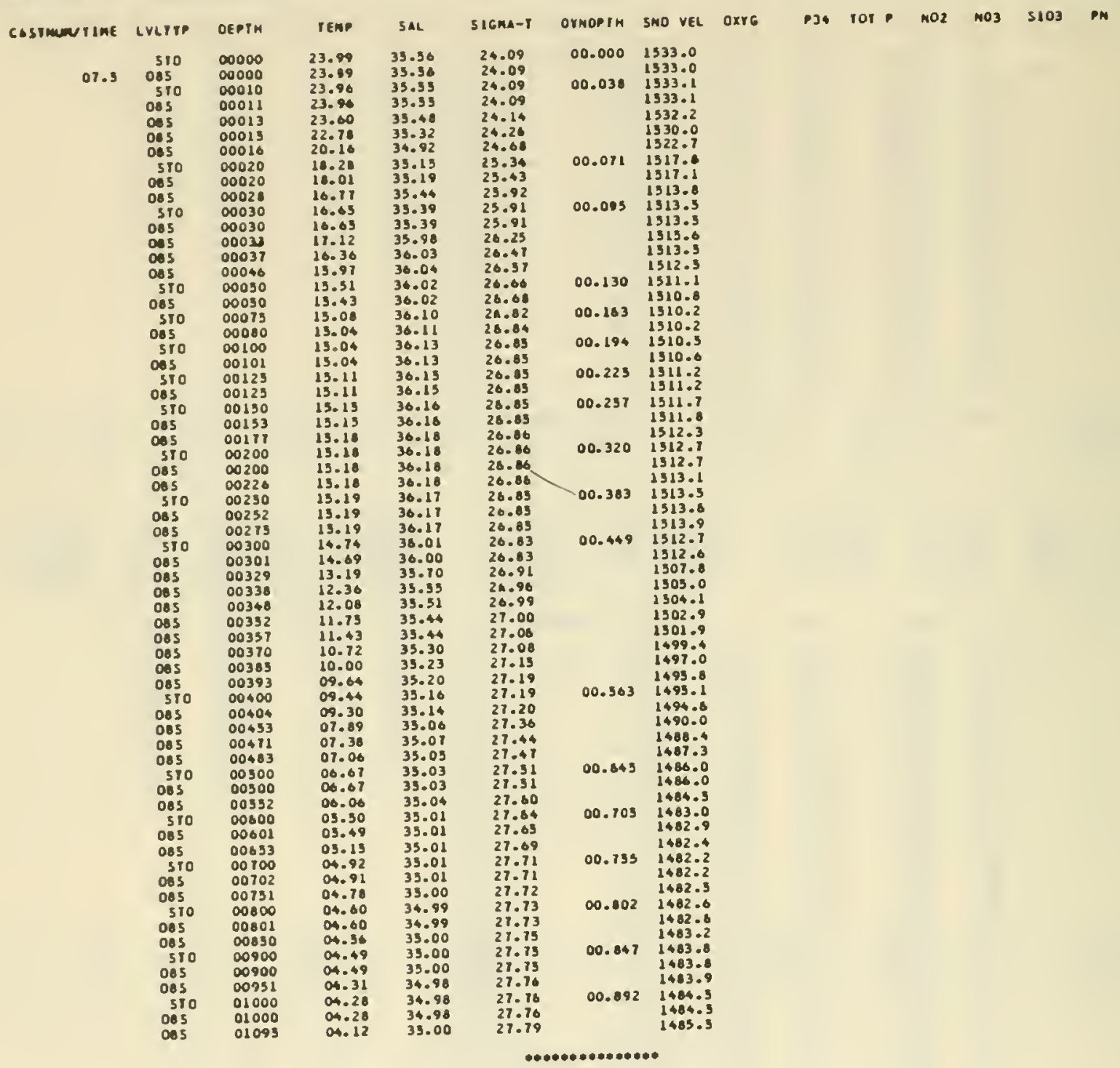


REFIO 31 C400 YEAR 197. CONSEC 0007 MONIH OB $\begin{array}{lrrrr}\text { LAT } & 36 & 09.5 \mathrm{~N} & \text { OAY } & 09 \\ \text { LONG } 072 & 49.0 \mathrm{ON} \text { HOUR } 16.5\end{array}$
BOTOP 02500

IEUP 25.0

SHIP EV WEI DULB 23.0
OATA USE I GAMONETR 1020.0 AREA OS CLLUO I/A

OIR HGT PER
24 O 2
SEA
CLIIR

WIVO-DIR is WIVO-SPO 08 WINO-FOR
INSI SID RECOROER IEN SO 1209 IRACE OL OL S SQUARE DURAIION 01.2 2 SOUAAE 2

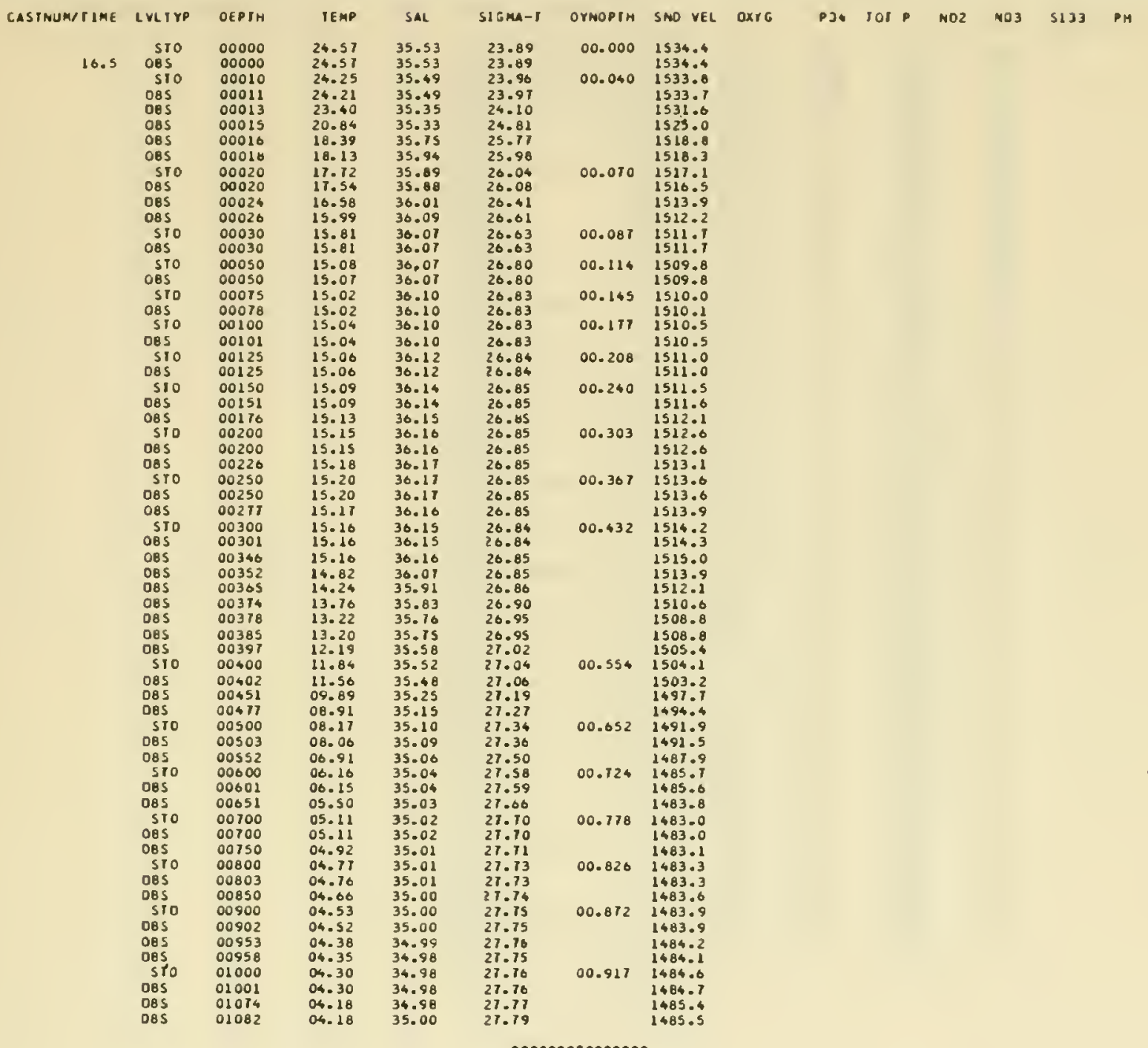




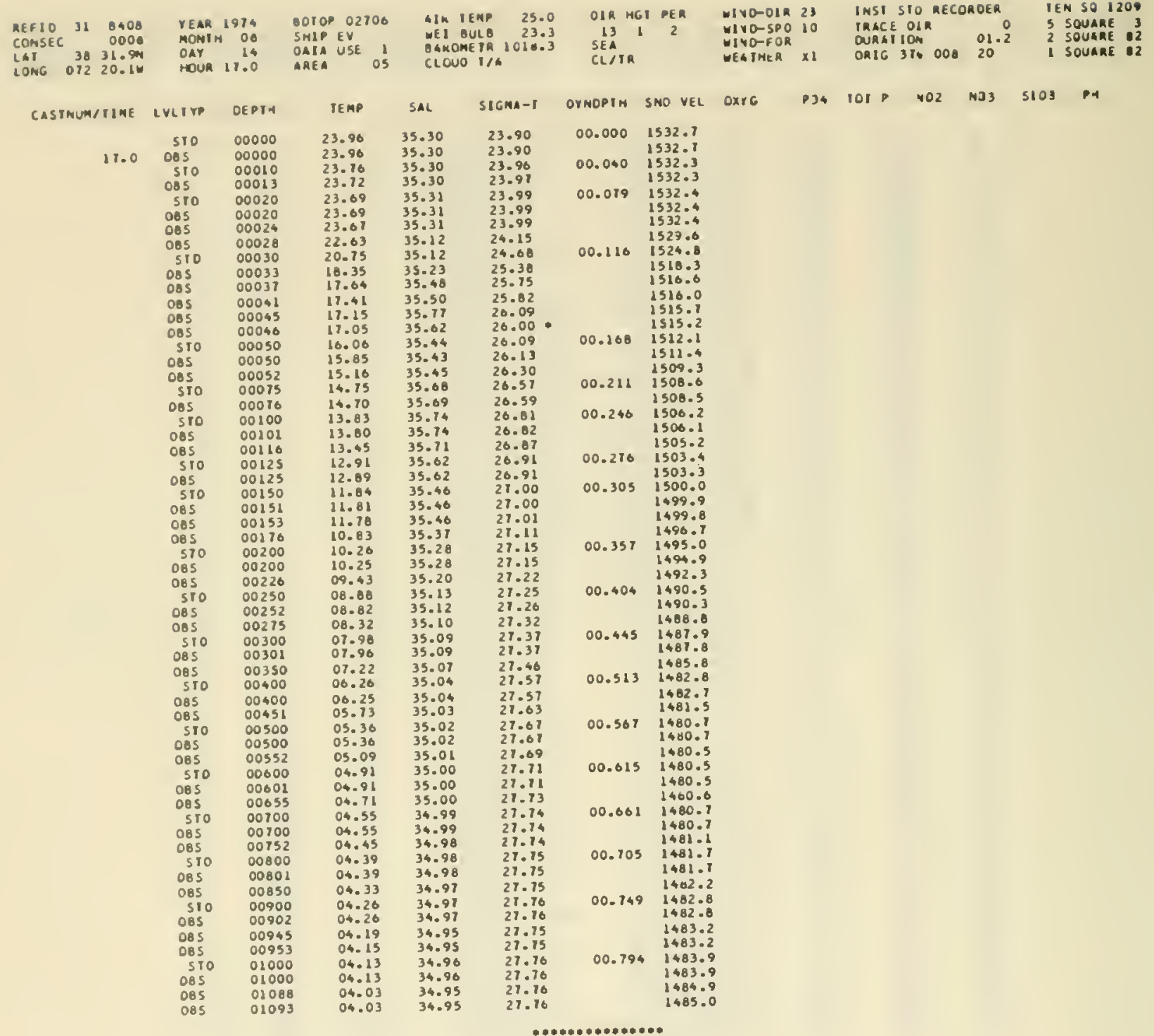


$\begin{array}{llll}\text { MEFIO } & 31 & 8408 & \text { YEAR } 1976 \\ \text { CONSEC } & 0009 & \text { MONTH O8 }\end{array}$ LAT $3840.5 N$ OAY 46
BOIDP 02304

SHIP EV

OATA USE I

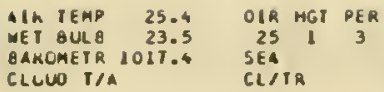

MIVDTIR 23

MIVD-SPD 13

HINO-FOR

MEATHER $X$
INST SIJ RELOROER

TEN SO 1209 OURAIION O1.1 2 SUUARE 3 ORIG 374009 3i I SOJARE 82

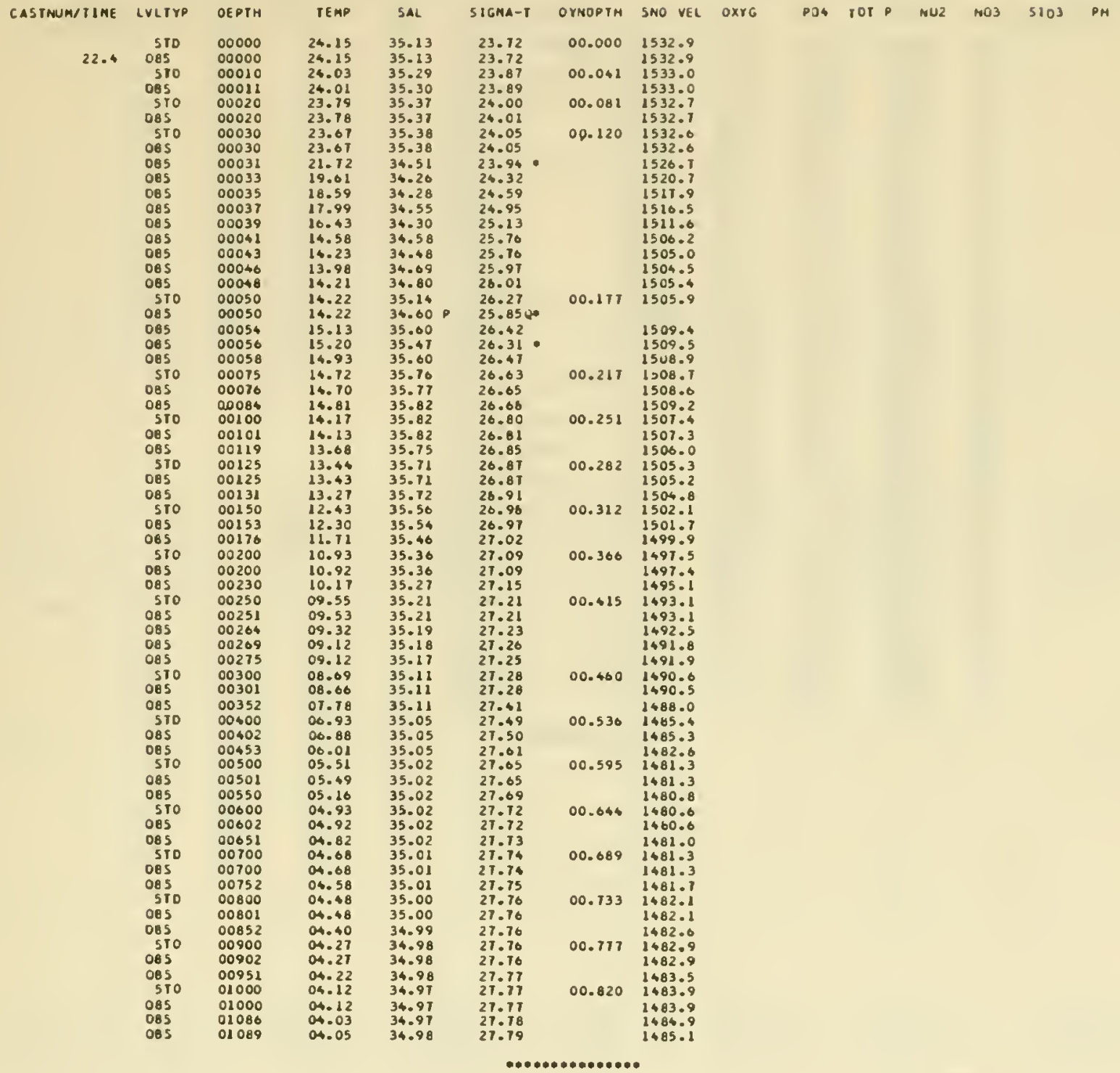




\begin{tabular}{|c|c|c|c|c|}
\hline AEF 10 & 31 & 0400 & YEAR 1914 & $8010 P \quad 00020$ \\
\hline CONSEC & & 0010 & MONIA OA & SHIP EV \\
\hline (4) & 30 & $51.7 \mathrm{~N}$ & OAY & OAIA USE \\
\hline IONG & oร 2 & 53.24 & 01.2 & AREA \\
\hline
\end{tabular}

II IE IEAP 24.0 OIR HGT PER

MET BUL 8 23.5

BA HONETR 1018.8

CLLUO T/A

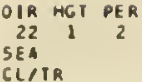

mivo-oin 23

YIVO-FJR

MEAINER XI
INSI SIU RECOROER

TRACE OIR

ORIG 37. 010
IEY 521209 SOUARE

SQUARE Bz

1 SOJARE D

\begin{tabular}{|c|c|c|c|c|c|c|c|}
\hline \multirow[t]{2}{*}{ CAST MUM/IIAE } & LVLJYP & OEPIH & IEAP & SAL & SIGMA-T & OYNOPTH & SNO VEL \\
\hline & 510 & 00000 & 23.73 & 34.64 & 23.47 & 00.000 & 1531.4 \\
\hline \multirow[t]{43}{*}{01.2} & 085 & 00000 & 23.73 & 34.04, & 23.47 & & 1531.6 \\
\hline & 570 & 00010 & 23.56 & 34.65 & 23.53 & 00.044 & 1531.1 \\
\hline & OAs & 00013 & 23.49 & 34.65 & 23.55 & & 1531.0 \\
\hline & STo & 00020 & 23.38 & 34.89 & 23.76 & 00.087 & 1531.1 \\
\hline & os & 00020 & 23.31 & 34.91 & 23.70 & & 1531.1 \\
\hline & 085 & 00022 & 19.84 & 33.82 & 23.92 & & 1526.1 \\
\hline & oas & 00026 & 15.77 & 34.41 & 25.37 & & 1509.5 \\
\hline & STo & 00030 & 15.63 & 34.38 & 25.37 & 00.121 & 1509.1 \\
\hline & 085 & 00030 & 15.63 & 34.30 & 25.31 & & 1509.1 \\
\hline & 065 & 00031 & 14.45 & 34.16 & 25.46 & & 1505.1 \\
\hline & oss & 00035 & 14.23 & 34.20 & 25.60 & & 1504.6 \\
\hline & Dos & 00037 & 13.76 & 34.49 & 25.86 & & 1503.4 \\
\hline & 085 & 00039 & 13.55 & 34.58 & 25.97 & & 1502.8 \\
\hline & 005 & 00041 & 13.64 & 34.14 & 26.08 & & 1503.3 \\
\hline & 085 & 00043 & 13.47 & 34.10 & 26.08 & & 1502.0 \\
\hline & oss & 00048 & 13.40 & 34.89 & 26.23 & & 2503.1 \\
\hline & 510 & 00050 & 13.70 & 35.05 & 26.29 & 00.164 & $1504 \cdot 3$ \\
\hline & 085 & 00050 & 13.86 & 35.09 & 26.30 & & 1504.7 \\
\hline & 005 & 00054 & 13.82 & 34.95 & 26.20 . & & 1504.4 \\
\hline & oes & 00056 & 13.39 & 34.86 & 26.22 & 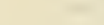 & 1502.0 \\
\hline & OQS & 00059 & 13.30 & 36.96 & 26.32 & & 1502.8 \\
\hline & 085 & 00061 & 13.00 & 35.25 & 26.44 & & 1504.8 \\
\hline & 085 & 00065 & 13.96 & 35.28 & 26.44 & & 1505.5 \\
\hline & 005 & 00069 & 13.68 & 35.37 & 26.50 & & 1504.7 \\
\hline & 510 & 00075 & 13.65 & 35.47 & 26.64 & 00.204 & 1504.8 \\
\hline & OAs & 00076 & 13.64 & 35.50 & 26.67 & & 1504.9 \\
\hline & 510 & 00100 & 13.52 & 35.60 & 26.17 & $00.23 \approx$ & 1505.0 \\
\hline & 085 & 00101 & 13.50 & 35.00 & 20.17 & & 1504.9 \\
\hline & 510 & 00125 & 12.89 & $\begin{array}{l}35.58 \\
35.58\end{array}$ & $\begin{array}{l}26.88 \\
26.89\end{array}$ & 00.270 & 1503.3 \\
\hline & Oes & $\begin{array}{l}00127 \\
00150\end{array}$ & $\begin{array}{l}12.85 \\
12.74\end{array}$ & $\begin{array}{l}35.58 \\
35.60\end{array}$ & $\begin{array}{l}26.89 \\
26.93\end{array}$ & 00.300 & $\begin{array}{l}1503.2 \\
1503.2\end{array}$ \\
\hline & $\begin{array}{l}510 \\
085\end{array}$ & 00152 & 12.73 & 35.00 & 20.93 & 0.00 & 1503.2 \\
\hline & 085 & 00117 & 12.55 & 35.59 & 26.96 & & 1503.0 \\
\hline & STo & 00200 & 11.00 & 35.46 & 27.00 & 00.357 & 1500.6 \\
\hline & 085 & 00202 & 11.11 & 35.45 & 21.01 & & 1500.3 \\
\hline & 085 & 00228 & 10.56 & 35.35 & 27.15 & & 1496.6 \\
\hline & STO & 00250 & 09.69 & 35.25 & 27.22 & 00.400 & 1493.7 \\
\hline & 085 & 00251 & 09.60 & 35.25 & 27.22 & & 1493.6 \\
\hline & oes & 00275 & 09.12 & 35.11 & 27.25 & & 1491.9 \\
\hline & 085 & 00279 & 08.14 & 35.16 & 27.29 & & 1490.5 \\
\hline & 510 & 00300 & 08.14 & 35.12 & 27.37 & 00.450 & $148 B .5$ \\
\hline & 005 & 00303 & 08.06 & 35.12 & 27.38 & & 1408.3 \\
\hline & 085 & 00350 & 07.50 & 35.10 & 27.45 & & 1486.9 \\
\hline & 085 & 00399 & 06.61 & 35.09 & 27.56 & & 1686.2 \\
\hline
\end{tabular}

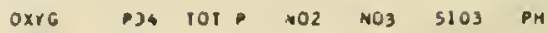


$\begin{array}{llr}\text { REFIU } & 31 & 8408 \\ \text { CONSEC } & 0011 \\ \text { LAI } & 39 & 02.2 \mathrm{~N}\end{array}$

LANG $07309.1 \mathrm{~N}$

YEAR 1974 MONIIH OB
OAY I HOUR 03.5
DOJOP OOOSB
SMID EV
OATA USE I

OATA USE

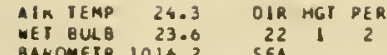
BAKONETR 1018.2

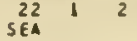

SLA IR
WIVO-OIR 27 WIYO-SDD IS WIVO-F $3 R$ MEATMER X

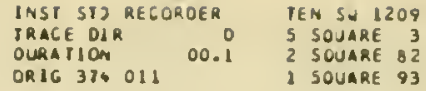

IEN SA 1209 00.1 S SOUARE 82 1 souare 93

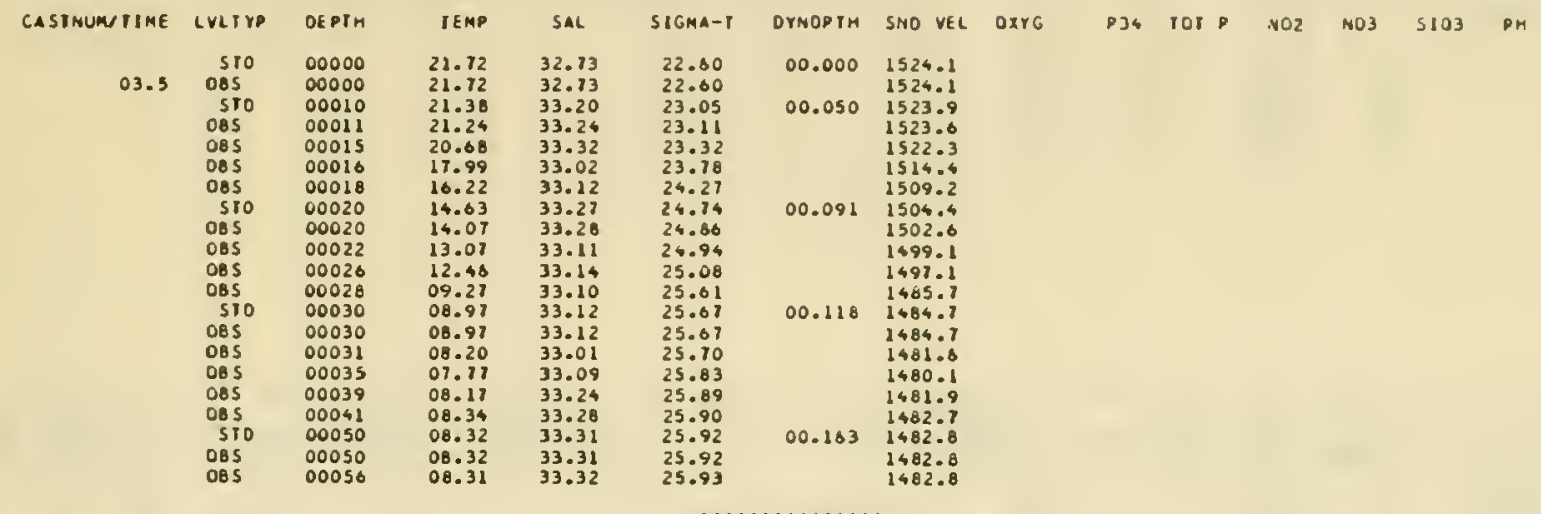

$\bullet \bullet \bullet \bullet \bullet \bullet * \bullet * \bullet \bullet \bullet \bullet$

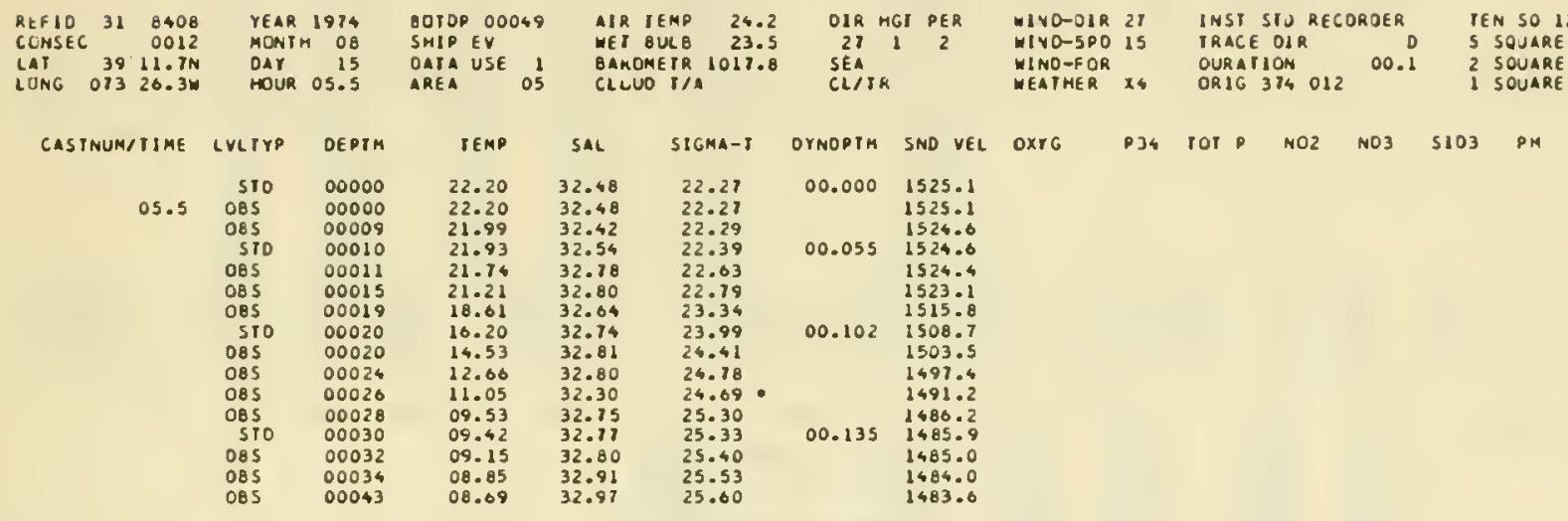

$\begin{array}{llrll}\text { REFIO } 31 & 8408 & \text { YEAR } 1974 \\ \text { CONSEC } & 0013 & \text { MONIH O } \\ \text { LAT } & 3921.7 N & \text { OAY } 15 \\ \text { LONG O73 } 43.7 \% & \text { MOUR O7.3 }\end{array}$
8010P 00031

SMIP EV

UATA USE

\section{AIK IEMP 22.8 OIR MGT PER IEI BULB 21.1 BAKOMETR 1019.2

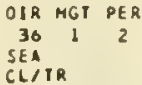

WINDEUIR is MIVO-SPO 13 WINO-FOR
INST STD RECOROER TRACE DIR O 5 SOUARE 3

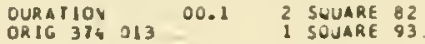

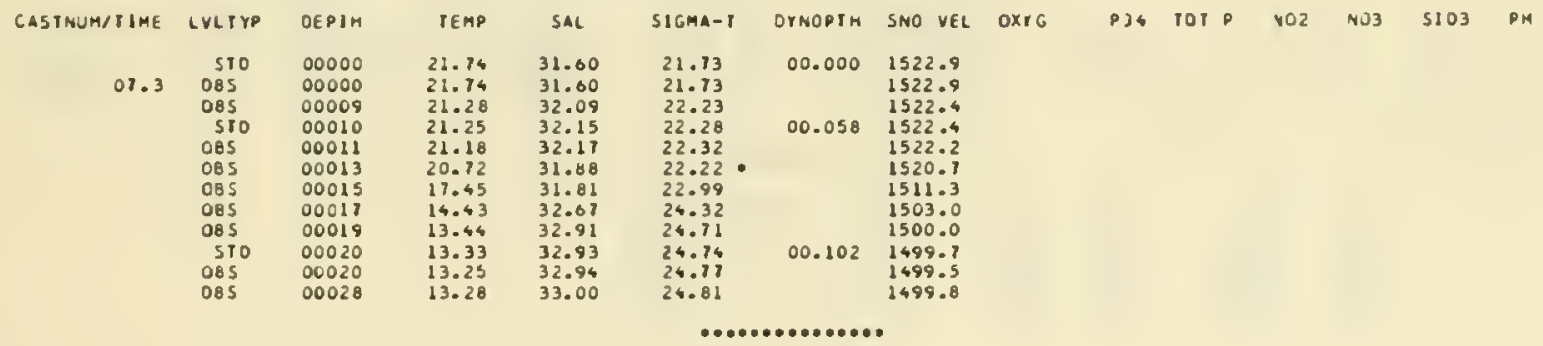




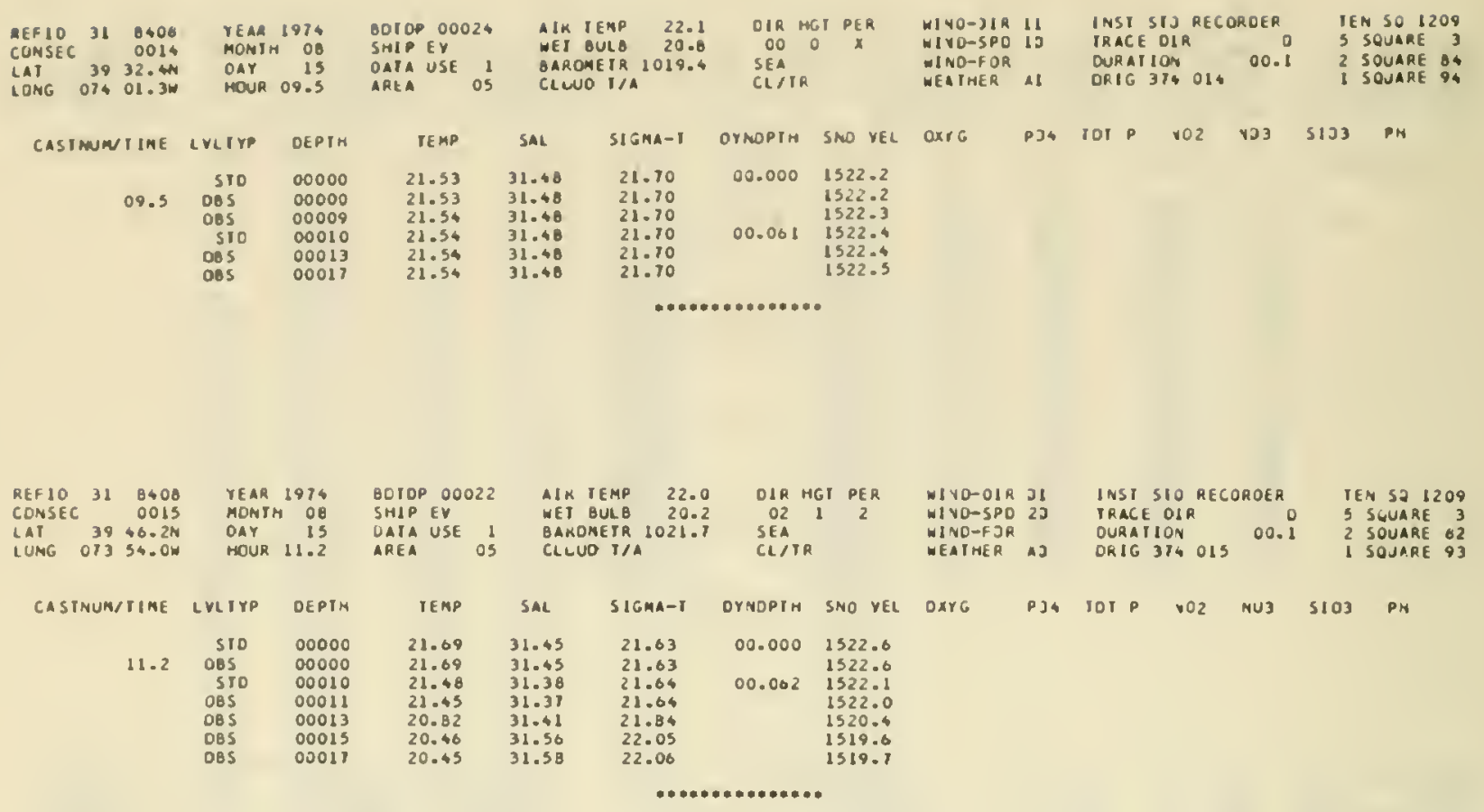

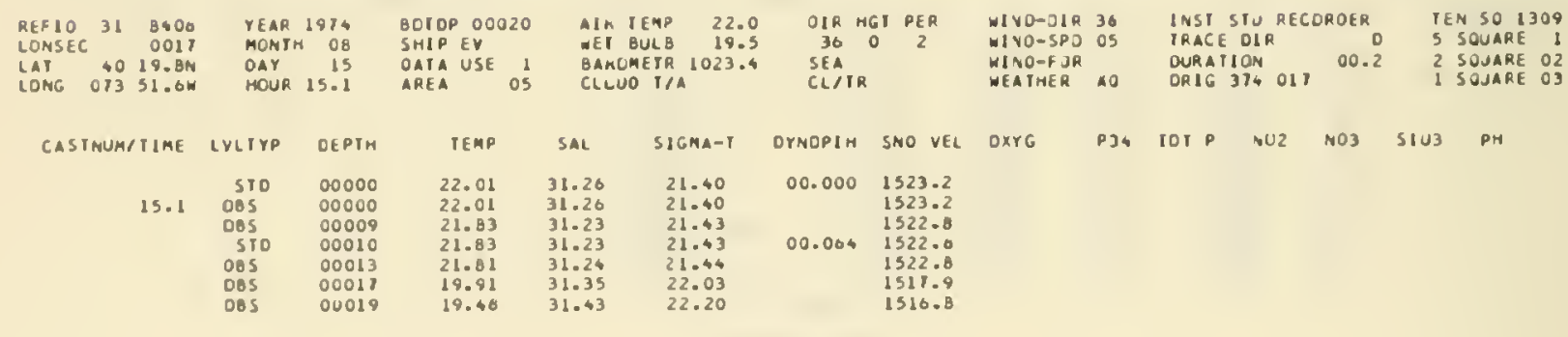

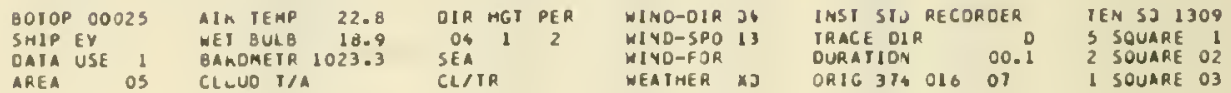

\begin{tabular}{|c|c|c|c|c|c|c|c|}
\hline CASTMUN/TIME & LVLIYP & OEPIN & TEMP & SAL & $S(G M A-1$ & OYMOPTH & SMO VEL \\
\hline & STO & 00000 & 21.05 & 31.20 & 21.62 & 00.000 & 1520.6 \\
\hline 13.1 & oos & 00000 & 21.05 & 31.20 & 21.62 & & 1520.0 \\
\hline & S10 & 00010 & 20.92 & 31.26 & 21.70 & 00.062 & 1520.5 \\
\hline & $\begin{array}{l}\text { OBS } \\
\text { OBS }\end{array}$ & $\begin{array}{l}00011 \\
00017\end{array}$ & $\begin{array}{l}20.85 \\
20.57\end{array}$ & $\begin{array}{l}31.32 \\
31.37\end{array}$ & $\begin{array}{l}21.76 \\
21.87\end{array}$ & & $\begin{array}{l}1520.3 \\
1519.7\end{array}$ \\
\hline & SID & 00020 & 20.57 & 31.37 & 21.87 & 00.122 & 1519.8 \\
\hline & 085 & 00020 & 20.57 & $31.3 \mathrm{~J}$ & 21.87 & & 1519.8 \\
\hline
\end{tabular}




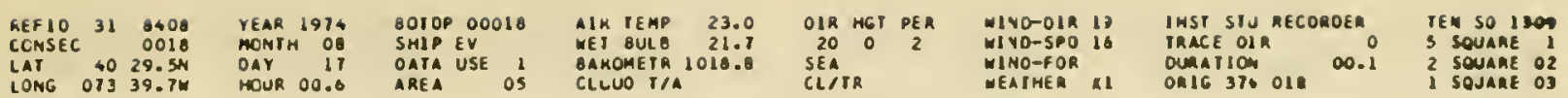

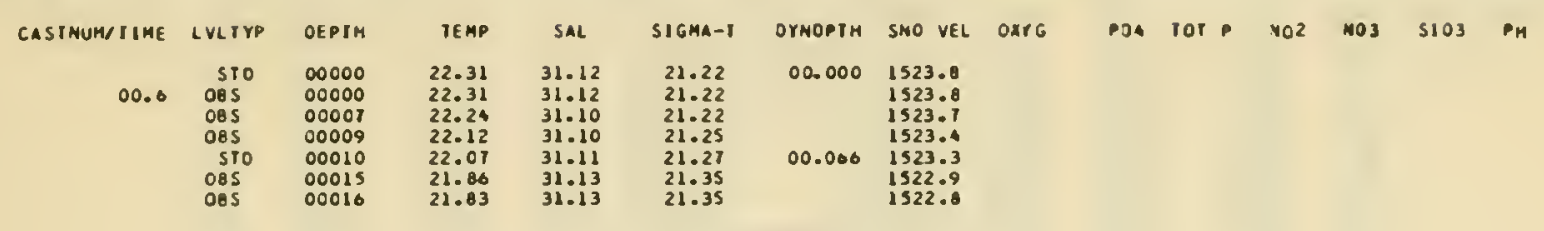

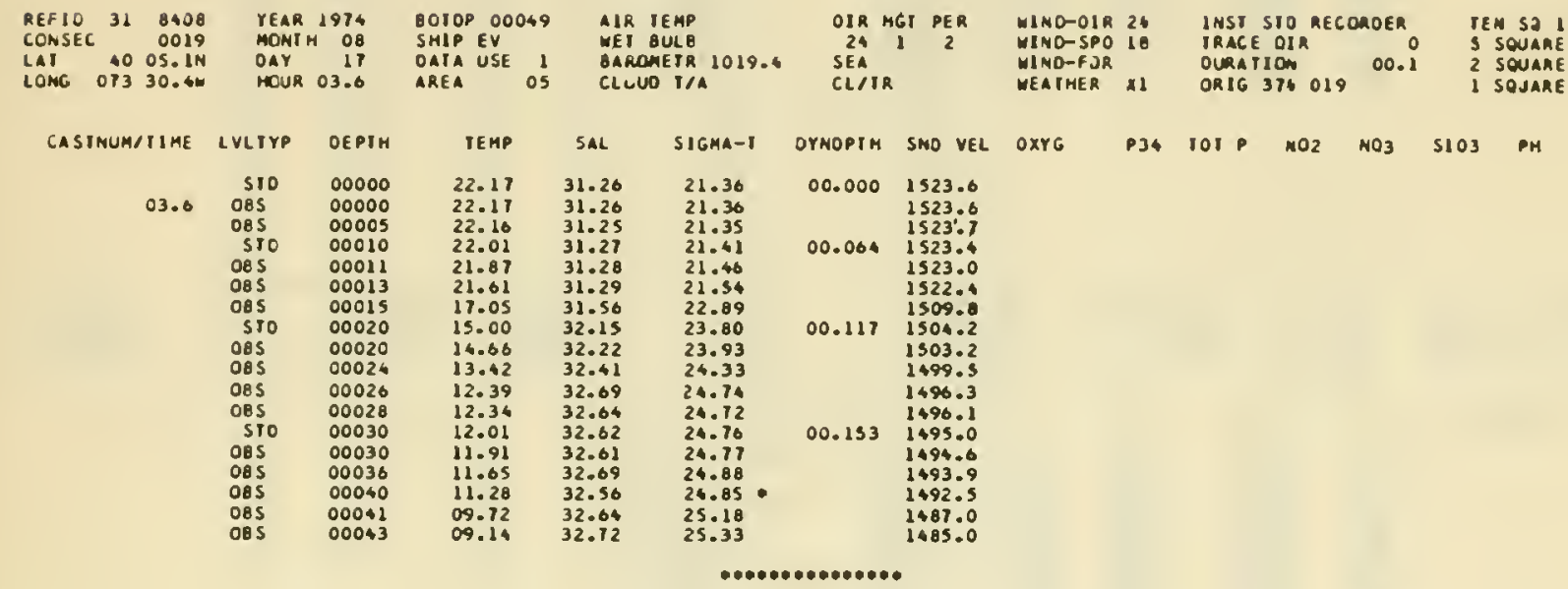

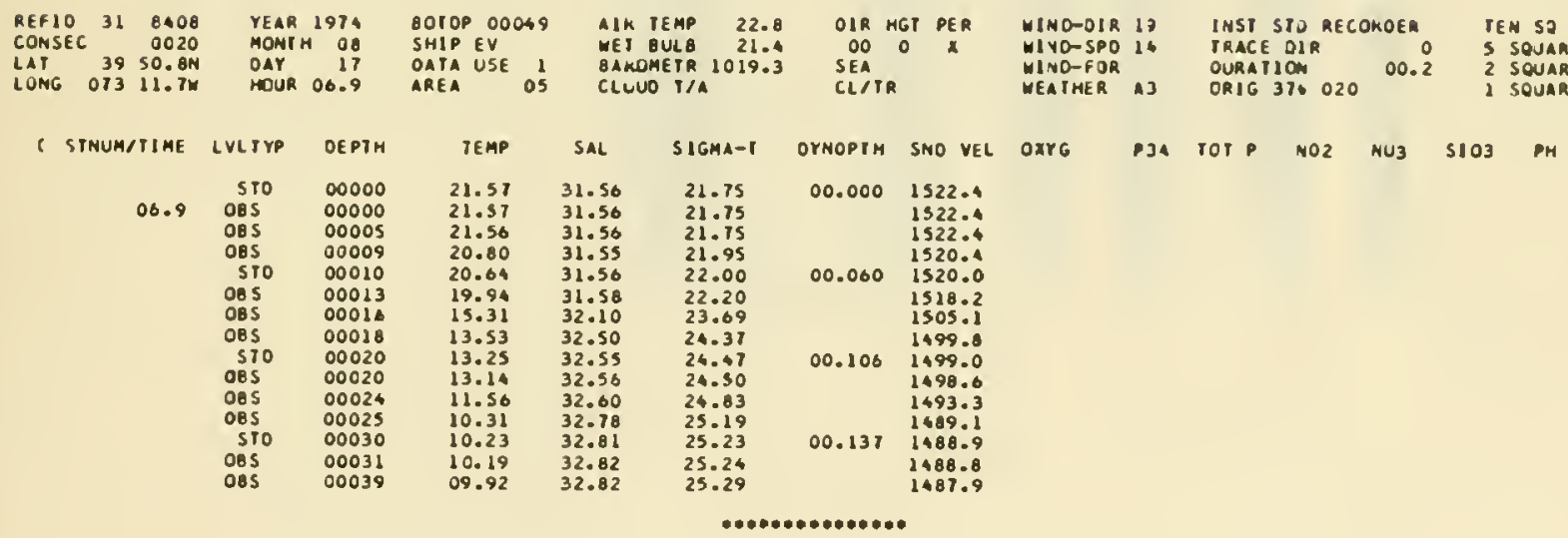




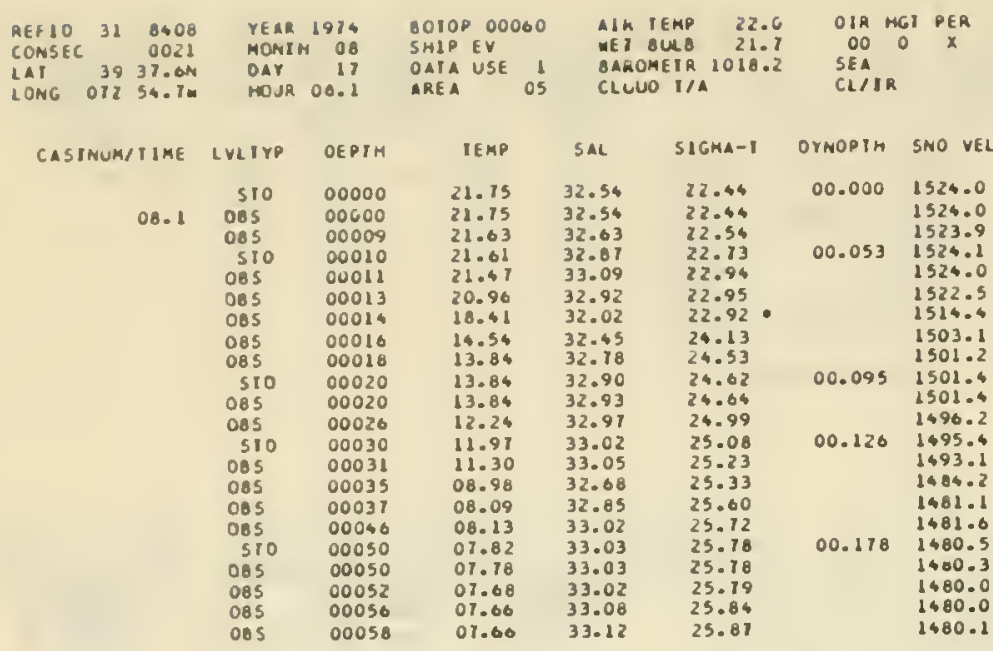

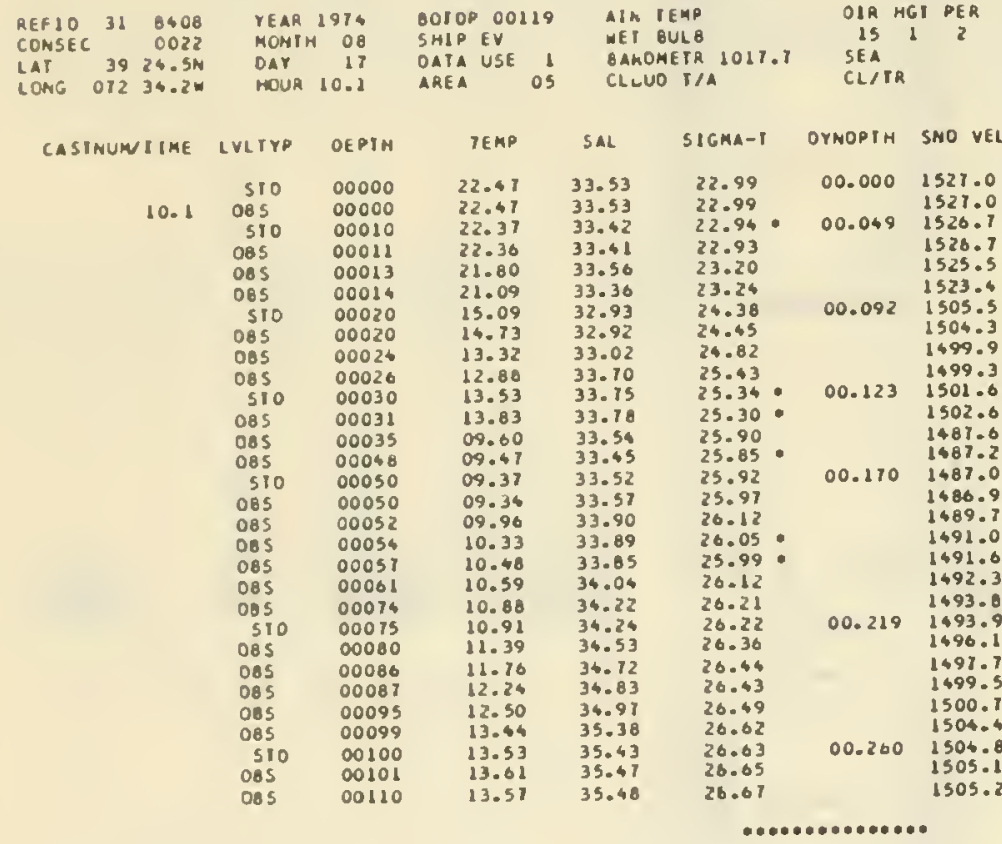

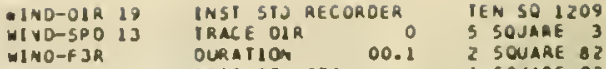
WINO-F3R OURMIOA
WEATHE 


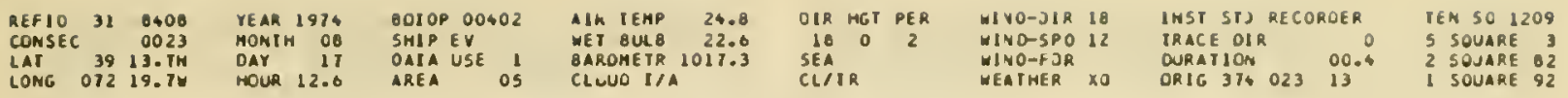

\begin{tabular}{|c|c|c|c|c|c|c|c|}
\hline \multirow{44}{*}{$\begin{array}{r}\text { CASIMUNI IME } \\
12.6\end{array}$} & LVLTYP & DEPIM & IEMP & SAL & SIGMA-1 & OYAOPTH & SNO VEL \\
\hline & STO & 00000 & 23.92 & 35.51 & 24.01 & 00.000 & 1532.8 \\
\hline & oes & 00000 & 23.92 & 35.51 & 26.07 & & 1532.8 \\
\hline & 510 & 00010 & 23.92 & 35.51 & $24.0 T$ & 00.039 & 1533.0 \\
\hline & obs & 00011 & 23.92 & 35.51 & 24.07 & & 1533.0 \\
\hline & 510 & 00020 & 23.90 & 35.51 & 24.08 & 00.077 & 2533.1 \\
\hline & oes & 00020 & 23.90 & 35.51 & 24.08 & & 1533.1 \\
\hline & 005 & 00022 & 23.66 & 35.50 & 24.14 & & 1532.5 \\
\hline & oss & 00026 & 23.53 & 35.40 & 24.10 & & 1532.2 \\
\hline & S10 & 00030 & 21.57 & 35.40 & 24.66 & 00.113 & 1521.3 \\
\hline & oes & 00030 & 21.57 & 35.40 & 24.66 & & 1527.3 \\
\hline & 085 & 00031 & 20.32 & 35.26 & 24.89 & & 1523.8 \\
\hline & 085 & 00033 & 19.70 & 35.60 & 25.32 & & 1522.6 \\
\hline & oos & 00043 & 19.09 & 35.69 & 25.54 & & 1521.1 \\
\hline & 085 & 00046 & 19.02 & 35.69 & 25.56 & & 1521.0 \\
\hline & $\$ 10$ & 00050 & 16.94 & 35.59 & 26.00 & 00.166 & 1514.9 \\
\hline & $\begin{array}{l}\text { oes } \\
\text { oos }\end{array}$ & $\begin{array}{l}00050 \\
00054\end{array}$ & $\begin{array}{l}16.70 \\
16.42\end{array}$ & $\begin{array}{l}35.58 \\
35.52\end{array}$ & $\begin{array}{l}26.05 \\
26.07\end{array}$ & & $\begin{array}{l}1514.2 \\
1513.3\end{array}$ \\
\hline & Des & $\begin{array}{l}00061 \\
00075\end{array}$ & $\begin{array}{l}15.38 \\
14.96\end{array}$ & $\begin{array}{l}35.68 \\
35.72\end{array}$ & $\begin{array}{l}20.43 \\
26.55\end{array}$ & & \\
\hline & $\begin{array}{c}510 \\
085\end{array}$ & $\begin{array}{l}00075 \\
00070\end{array}$ & $\begin{array}{l}14.96 \\
14.93\end{array}$ & $\begin{array}{l}35.12 \\
35.13\end{array}$ & $\begin{array}{l}26.55 \\
26.57\end{array}$ & 00.211 & $\begin{array}{l}1509.4 \\
1509.3\end{array}$ \\
\hline & $\begin{array}{l}085 \\
085\end{array}$ & $\begin{array}{l}00070 \\
00086\end{array}$ & $\begin{array}{l}14.93 \\
14.91\end{array}$ & $\begin{array}{l}35.73 \\
35.83\end{array}$ & $\begin{array}{l}26.57 \\
26.65\end{array}$ & & $\begin{array}{l}1509.3 \\
1509.5\end{array}$ \\
\hline & $\begin{array}{l}005 \\
\text { STO }\end{array}$ & $\begin{array}{l}00086 \\
00100\end{array}$ & 14.55 & 35.82 & 26.12 & 00.246 & $\begin{array}{l}1509.5 \\
1508.6\end{array}$ \\
\hline & $\begin{array}{l}510 \\
085\end{array}$ & 00103 & 14.45 & 35.81 & $26 \cdot 13$ & & 1508.3 \\
\hline & $\begin{array}{l}085 \\
\text { STo }\end{array}$ & 00125 & 13.59 & 35.68 & 26.62 & 00.279 & 1505.7 \\
\hline & oss & 00125 & 13.57 & 35.08 & 26.82 & & 1505.7 \\
\hline & 005 & 00142 & 13.16 & 35.66 & 26.89 & & 1504.6 \\
\hline & STO & 00150 & 12.69 & 35.61 & 26.95 & 00.310 & 1503.0 \\
\hline & 085 & 00150 & 12.69 & 35.61 & 26.95 & & 1503.0 \\
\hline & 085 & 00176 & 12.01 & 35.50 & 26.99 & & 1501.0 \\
\hline & 085 & 00198 & 11.29 & 35.42 & $2 T .07$ & & $1+98.8$ \\
\hline & STO & 00200 & 11.21 & 35.40 & 27.07 & 00.365 & 1498.5 \\
\hline & 085 & 00202 & 11.05 & 35.36 & 27.07 & & 1497.9 \\
\hline & 085 & 00215 & 10.40 & 35.29 & 27.13 & & 1495.1 \\
\hline & 085 & 00225 & 10.06 & 35.25 & $2 T \cdot 16$ & & 1494.6 \\
\hline & 085 & 00243 & 09.68 & 35.21 & 27.19 & & 1493.5 \\
\hline & 085 & 00245 & 09.40 & 35.11 & 27.21 & & 1492.4 \\
\hline & STo & 00250 & 09.31 & 35.17 & 27.22 & 00.414 & 1492.2 \\
\hline & OBS & 00255 & 09.21 & 35.16 & 27.23 & & 1491.9 \\
\hline & 085 & 00275 & 00.14 & 35.13 & 27.28 & & 1490.4 \\
\hline & STO & 00300 & 08.39 & 35.11 & 27.32 & 00,450 & 1489.5 \\
\hline & 085 & 00300 & 08.39 & 35.11 & 27.32 & & 1489.5 \\
\hline & 085 & 00328 & 07.65 & 35.05 & 27.39 & & 1487.0 \\
\hline & 085 & 00352 & 07.10 & 35.06 & 27.47 & & 1485.3 \\
\hline & 085 & 00393 & 06.05 & 35.07 & $2 T .52$ & & 1485.0 \\
\hline & OBS & 00395 & 06.85 & 35.08 & 27.52 & & 1485.1 \\
\hline
\end{tabular}


NOOCSTAIIONOATA

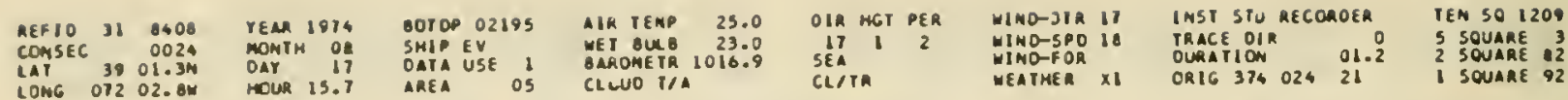

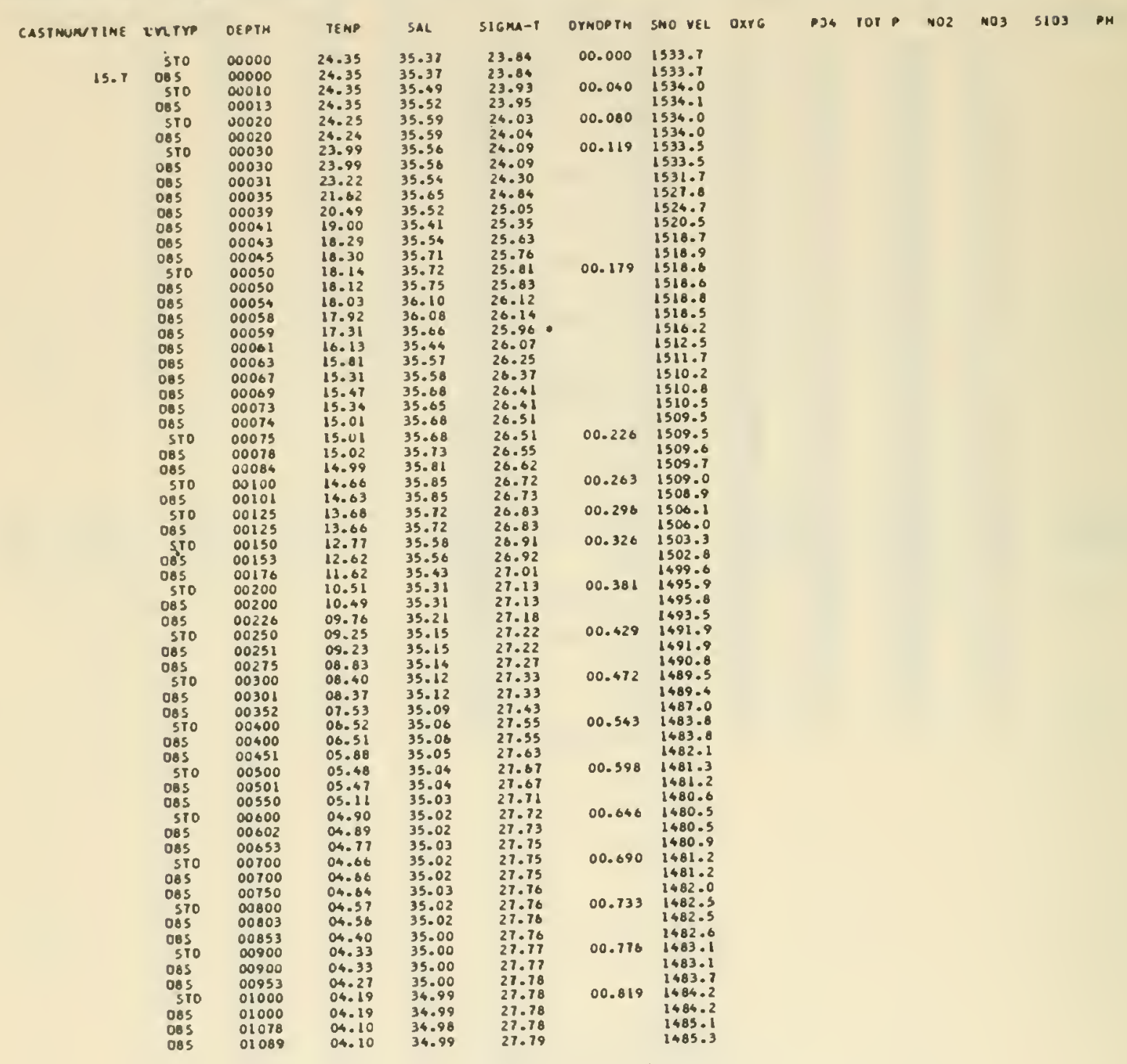


NOC SIA I ION OAIA

$\begin{array}{lrr}\text { REF 10 } 31 & 0406 \\ \text { CCNSEC } & 0025 \\ \text { LAT } & 38 & 52.0 N \\ \text { LONG O71 } & 47.96\end{array}$

YEAR 1974 BOTOP O2500
MONTH OO SHIPEV
OAY IT OATA USE 1

MOUR 19.1
OATA USE 1
AIT IEMP 26.0 OIR HGT PEA MEI OULS 23.0 15 02 BAROMETR 1014.2

OIR HGT PEA
SE O 2
CLITR

WIVO-DIR IS MIVOSPO 19 MINO-FOR WEITHEA $X$
INST SIJ RECOROER $\begin{array}{lll}\text { DURAIION } & 02.0 & 2 \\ \text { OAIG } 370025 & 25 & 1\end{array}$ IEN 501209
SIGMA-T OYNOPIM SNO VEL OXYG PJ4 JOIP NO2 NO3 5103 PH

.

\section{IEMP}

SAL

23. 81
24.73
24.73

24.65

24.63

24.56

24.39

22.59

22.59

21.82

21.32
21.00

19.79

17.27

17.09

16.53

10.68

15.70
15.54

15.54
15.47

15.19

15.45

15.45
15.38

15.14

14.34

13.50

13.49

12.02

12.70

11.81

11.06

11.05
10.28

10.28
09.53

09.50

08.94

08.71

08.10

08.08

06.62

06.28

06.25

05.74

05.29
05.26

05.03

04.88

04.86

04.72

04.64

04.55

04.49

04.48

04.35

04.24

04.19

04.12

04.12
0.10

04.03

04.03
23.82

23.02

23.84

23.89

24.03

24.75

24.75

25.29

25.20 .

25.64

25.96
25.99

26.14

26.22

26.32

26.4

26.28

26.47

26.55

26.54

26.59

26.74
26.75

26.87

26.87

26.93

27.02

27.10

27.1

27.15
27.20

27.20

27.27

27.28

27.35

27.36

27.52

27.59

27.60

21.65

27.69

27.69

27.11

27.73

27.73

27.75

27.76

27.16

27.76

27.77

27.77

27.78

27.78

27.18

27.79

27.79

27.79

27.78

27.79
27.80
$00.000 \quad 1534.7$

00.0411534 .7

$00.002 \quad 1534.0$ 1534.3 1533.9

$00.118 \quad 1530.5$ 1530.5 1527 1526.5 1523.5

$00.171 \quad 1516.0$

1515.4

1513.8

151408

511.5

1510.7

2510.0

$00.216 \quad 1511.1$

1511.1

510.1

$00.252 \quad 1507.9$

1507.8

$00.284 \quad 1505.5$

1505.5

00.3141503 .5

1503.3

00.369

1490.0

1495.5

$00.418 \quad 1493.0$ 1491.1 1490.3

00.4612488 .3

488.2

$00.529 \quad 1482.0$

1482.8

00.581281 .5

1480.5

$00.627 \quad 1460.5$

1480.5

1480.7

$00.670 \quad 1481.1$

481.1

$00.113 \quad 148201$

1482.1

1482

$00.755 \quad 1482.7$

1482.8

$00.797 \quad 1483.4$

483.9

483.9

1484.0

484.9

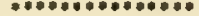




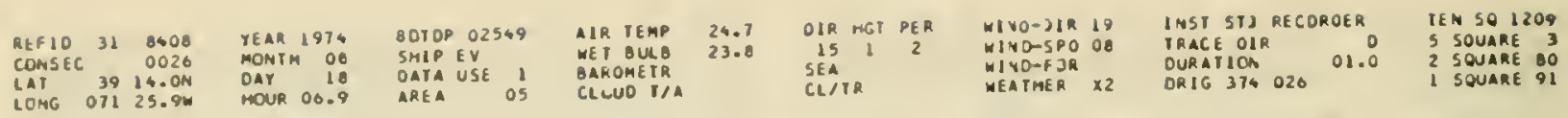

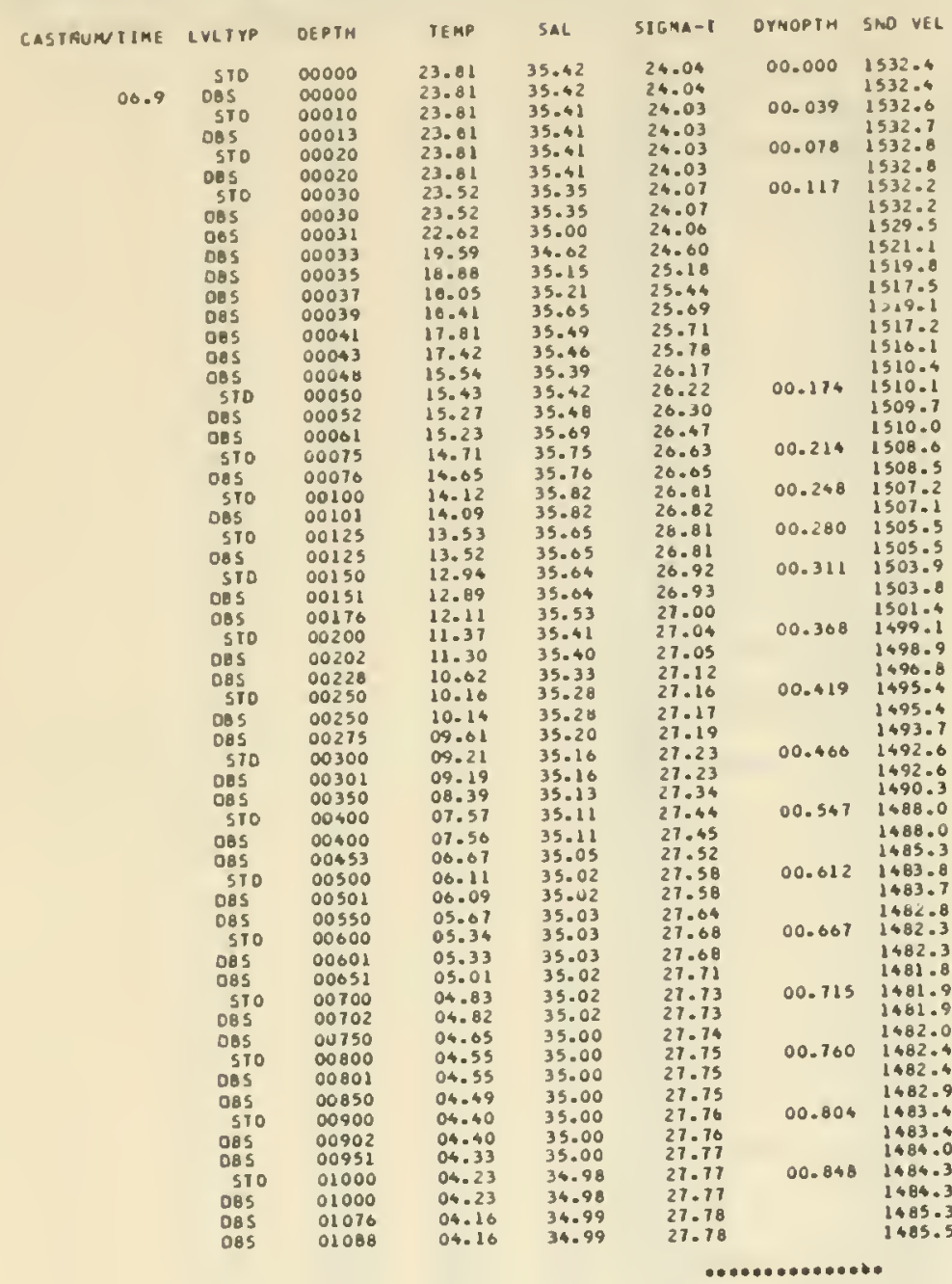




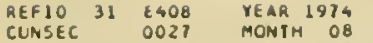
LAT $3925.3 \mathrm{~N}$ MAY $\begin{array}{lrrr}39 & 25.3 \mathrm{~N} & \text { OAY } 18 \\ \text { LONG OT1 } 39.8 \mathrm{~W} & \text { MOUR } 09.6\end{array}$

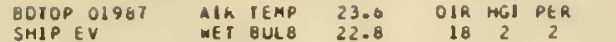
SHIP EV

OATA USE BAMOMETR 1009.9 CLLUO I/A

OIR HGI PER
I8
SEA
CL/IA

$M I V O-D I R \quad 51$ WIVO-SPD 1

WIND-FOR

MESTHER XZ
INST STJ KECOKOEA TEN SO 1209 TRACE OIR OA S SOUARE 3 OURATION ON OO.5 2 SOUARE 80
CASINUMJIME LVITYP OEPIH

\begin{tabular}{|c|c|c|}
\hline \multirow{3}{*}{09.6} & sto & 00000 \\
\hline & 085 & 00000 \\
\hline & $\$ 10$ & 00010 \\
\hline & $\begin{array}{l}\text { UBS } \\
\text { SI D }\end{array}$ & $\begin{array}{l}00011 \\
00020\end{array}$ \\
\hline & OBS & 00022 \\
\hline & $\begin{array}{l}\text { OBS } \\
\text { SIO }\end{array}$ & $\begin{array}{l}00028 \\
00030\end{array}$ \\
\hline & oas & 00031 \\
\hline & o8s & 00033 \\
\hline & 085 & 00031 \\
\hline & 085 & 00039 \\
\hline & 085 & 00045 \\
\hline & 510 & 00050 \\
\hline & 085 & 00050 \\
\hline & 085 & 00052 \\
\hline & DBS & 00054 \\
\hline & 085 & 00056 \\
\hline & 085 & 00061 \\
\hline & 085 & 00007 \\
\hline & 510 & 00075 \\
\hline & D8S & 00076 \\
\hline & STO & 00100 \\
\hline & OBS & 00103 \\
\hline & 085 & 00110 \\
\hline & 510 & 00125 \\
\hline & 085 & 00125 \\
\hline & SIO & 00150 \\
\hline & 085 & 00151 \\
\hline & D85 & 00276 \\
\hline & STO & 00200 \\
\hline & 085 & 00202 \\
\hline & 085 & 00226 \\
\hline & sto & 00250 \\
\hline & D8S & 00251 \\
\hline & 085 & 00215 \\
\hline & 510 & 00300 \\
\hline & D8S & 00301 \\
\hline & 085 & 00352 \\
\hline & $\begin{array}{l}\text { S10 } \\
085\end{array}$ & $\begin{array}{l}00400 \\
00400\end{array}$ \\
\hline & $\begin{array}{l}085 \\
\text { STO }\end{array}$ & $\begin{array}{l}00451 \\
60500\end{array}$ \\
\hline & 085 & 00503 \\
\hline & 085 & 00550 \\
\hline & STO & 00600 \\
\hline & 085 & 00604 \\
\hline & 085 & 00651 \\
\hline & 510 & 00700 \\
\hline & 085 & 00700 \\
\hline & 085 & 00750 \\
\hline & 510 & 00800 \\
\hline & DBS & 00801 \\
\hline & 085 & 00850 \\
\hline & 510 & 00900 \\
\hline & OBS & 00900 \\
\hline & 085 & 00951 \\
\hline & OBS & 00998 \\
\hline & 510 & 01000 \\
\hline & OBS & 01000 \\
\hline & oBs & 01071 \\
\hline & 085 & 01089 \\
\hline
\end{tabular}

\begin{tabular}{|c|c|}
\hline IEMP & SAL \\
\hline 23.98 & 35.48 \\
\hline 23.98 & 35.48 \\
\hline 23.99 & 35.69 \\
\hline 23.99 & 35.69 \\
\hline 23.98 & 35.40 \\
\hline 23.94 & 35.45 \\
\hline $\begin{array}{l}23.78 \\
23.63\end{array}$ & $\begin{array}{l}35.49 \\
35.48\end{array}$ \\
\hline 23.69 & 35.48 \\
\hline 21.75 & 35.53 \\
\hline 20.91 & 35.74 \\
\hline 19.24 & 35.50 \\
\hline 17.39 & 35.48 \\
\hline 10.53 & 35.41 \\
\hline 16.48 & 35.47 \\
\hline 16.40 & 35.62 \\
\hline 16.29 & 35.65 \\
\hline 16.31 & 35.72 \\
\hline 15.72 & 35.51 \\
\hline 15.33 & 35.65 \\
\hline 14.69 & 35.73 \\
\hline 14.81 & 35.74 \\
\hline 14.46 & 35.77 \\
\hline $\begin{array}{l}14.27 \\
13.66\end{array}$ & $\begin{array}{l}35.77 \\
35.68\end{array}$ \\
\hline $\begin{array}{l}13.66 \\
13.35\end{array}$ & 35.07 \\
\hline 13.34 & 35.67 \\
\hline 12.65 & 35.60 \\
\hline 12.61 & 35.60 \\
\hline 12.06 & 35.53 \\
\hline 11.52 & 35.46 \\
\hline 11.45 & 35.45 \\
\hline 10.53 & 35.32 \\
\hline 09.94 & 35.23 \\
\hline 09.92 & 35.23 \\
\hline 09.46 & 35.18 \\
\hline 09.23 & 35.20 \\
\hline 09.21 & 35.20 \\
\hline 08.21 & 35.10 \\
\hline $\begin{array}{l}07.40 \\
07.39\end{array}$ & $\begin{array}{l}35.08 \\
35.08\end{array}$ \\
\hline $\begin{array}{l}06.53 \\
05.83\end{array}$ & $\begin{array}{l}35.05 \\
35.05\end{array}$ \\
\hline 05.19 & 35.05 \\
\hline 05.36 & 35.03 \\
\hline C5.OB & 35.02 \\
\hline $\begin{array}{l}05.04 \\
04.92\end{array}$ & $\begin{array}{l}35.02 \\
35.01\end{array}$ \\
\hline 04.17 & 35.01 \\
\hline 04.77 & 35.01 \\
\hline 04.60 & 35.00 \\
\hline 04.48 & 35.00 \\
\hline 04.48 & 35.00 \\
\hline 04.38 & 34.99 \\
\hline 04.30 & 34.99 \\
\hline 04.30 & 34.99 \\
\hline 04.28 & 35.00 \\
\hline 04.24 & 34.98 \\
\hline 04.24 & 34.99 \\
\hline 04.24 & 34.99 \\
\hline 04.13 & 34.98 \\
\hline 04.13 & 34.99 \\
\hline
\end{tabular}

OYNJPIM SNO VEL OXYG

Pos

00.000

1532.9

24.03

24.03

24.03

00.0391533.

1533.1
24.04

$29.02 \quad 1533.2$

$24.10 \quad 00.117 \quad 1532.9$

24.18

24.18

24.71

25.08

25.36

25.81
20.00

20.02

26.15

26.20

26.25

26.27

26.42
26.57

20.60

26.70

26.74

26.80

20.80
20.80

26.80
26.95

26.95

26.95

27.01

27.06

27.08

27.13
27.16

27.16

27.16
21.20

27.20
27.26

27.26
27.26

27.34

27.45

27.54
27.63

27.04

27.08

27.11

27.13

27.13

27.14

27.16
27.76

21.16

27.7

27.77

27.78

27.77

27.77

27.77
27.78

27.78
27.79

1532.3

1528.0
1526.2

1521.3

1516.0

$00.175+1513.5$

1513.4

1513.1

1513.3

1511.4

1510.3

$00.219 \quad 1509.2$

1505.0

00.2551508 .2

1507.7

$00.288 \quad 1504.9$

1504.9

00.3111502 .9

1502.8

$00.373 \quad 1501.2$

1494.4

1498.4

$00.424 \quad 1494.6$

1494.5

1493.2

1492.1

1492.1

1489.6

$60.550 \quad 1487.3$

484.7

$00.613 \quad \begin{array}{ll}1484.7 \\ 1482.7\end{array}$

1482.0

$00.063 \quad 1481.0$

1401.2

$00.709 \quad 1481.4$

1461.0

1481.6

$00.154 \quad 1482.1$

1482.1

1482.5

$00.198 \quad 1483.0$

1483.0

1483.0

00.8411484 .4

1484.4

1484.4

1465.4 


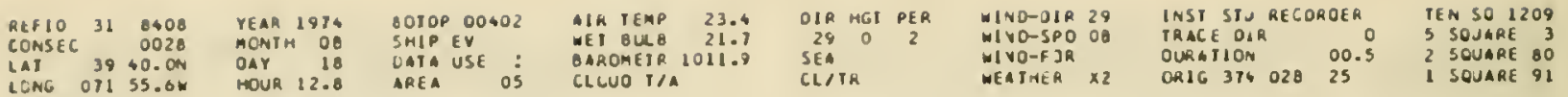

\begin{tabular}{|c|c|c|c|c|c|c|c|}
\hline \multirow[t]{2}{*}{ CASINUA/IITE } & LVLIYP & OEPIM & IEMP & SAL & SIGMA-T & OYNOPIM & SNO VEL \\
\hline & sto & 00000 & 22.84 & 34.57 & 23.67 & 00.400 & 1529.1 \\
\hline \multirow[t]{49}{*}{12.8} & 005 & $\infty 0000$ & 22.84 & 34.57 & 23.67 & & 1529.1 \\
\hline & 005 & 00005 & 22.83 & 34.56 & 23.07 & & 1529.1 \\
\hline & 085 & 00009 & 22.93 & 34.77 & 23.80 & & 1529.1 \\
\hline & STO & 00010 & 23.04 & 34.88 & 23.85 & 00.041 & 1530.1 \\
\hline & oes & 00015 & 23.27 & 35.28 & 24.09 & & 1531.2 \\
\hline & OBS & 00016 & 23.06 & 35.22 & 24.10 & & 1530.6 \\
\hline & STO & 00020 & 22.90 & 35.11 & 24.07 & 00.081 & 1530.2 \\
\hline & oes & 00020 & 22.57 & 35.08 & 24.14 & & 1529.3 \\
\hline & obs & 00022 & 20.92 & 34.98 & 26.52 & & 1525.0 \\
\hline & oos & 00024 & 19.07 & 35.17 & 25.15 & & 1520.1 \\
\hline & oes & 00026 & 18.59 & 34.85 & 25.03 & & 1518.4 \\
\hline & 510 & 00030 & 12.60 & 33.00 & 24.91 & 00.116 & 1498.2 \\
\hline & oes & 00030 & 12.00 & 33.00 & 24.91 & & 1498.2 \\
\hline & oss & 00033 & 10.43 & 32.92 & 25.28 & & 1489.8 \\
\hline & 085 & 00035 & 09.34 & 33.29 & 25.75 & & 1486.3 \\
\hline & oes & 00037 & 09.49 & 33.49 & 25.68 & & 1407.2 \\
\hline & oes & 00045 & 10.62 & 33.95 & 26.05 & & 1492.0 \\
\hline & Des & 00046 & 11.49 & 34.14 & 26.04 & & 1495.4 \\
\hline & 085 & 00048 & 11.76 & 34.16 & 26.02 & & 1496.4 \\
\hline & 510 & 00050 & 11.95 & 34.35 & 20.11 & 00.106 & 1497.3 \\
\hline & oes & 00050 & 12.04 & 34.42 & 26.15 & & 1497.1 \\
\hline & oes & 00058 & 12.55 & 34.67 & 26.25 & & 1499.9 \\
\hline & 085 & 00003 & 12.42 & 34.16 & 26.34 & & 1499.6 \\
\hline & 085 & 00005 & 12.84 & 35.07 & 26.50 & & 1501.5 \\
\hline & OBS & 00001 & 13.16 & 35.16 & 26.51 & & 1502.6 \\
\hline & 085 & 00069 & 13.11 & 35.19 & 26.52 & & \\
\hline & oBs & 00073 & 13.52 & 35.59 & 20.76 & & 1304.5 \\
\hline & STO & 00075 & 13.67 & 35.50 & 20.60 & 00.201 & 1504.9 \\
\hline & 085 & 00076 & 13.79 & 35.43 & 26.58 & & 1505.3 \\
\hline & STO & 00100 & 14.11 & 15.13 & 20.14 & 00.242 & $1507 \cdot 1$ \\
\hline & oes & 00101 & 14.13 & 35.14 & 20.75 & & 1501.2 \\
\hline & 085 & 00110 & 14.30 & 35.84 & 20.19 & & 1508.0 \\
\hline & OBS & 00123 & 13.27 & 35.65 & 26.86 & & 1504.6 \\
\hline & $\$ 10$ & 00125 & 13.23 & 35.68 & 26.89 & 00.274 & 1504.5 \\
\hline & OBS & 00125 & 13.23 & 35.68 & 26.89 & & 1504.5 \\
\hline & Oes & 00127 & 23.27 & 35.05 & 26.86 & & 1504.7 \\
\hline & 510 & 00150 & 12.56 & 35.59 & 20.96 & 00.303 & 1502.6 \\
\hline & OBS & 00150 & 12.56 & 35.59 & 26.96 & & 1502.6 \\
\hline & oes & 00170 & 11.92 & 35.50 & 27.01 & & 1500.7 \\
\hline & 510 & 00200 & 11.03 & 35.39 & 27.09 & 00.358 & 1497.9 \\
\hline & oss & 00206 & 10.86 & 35.37 & 27.11 & & 1497.3 \\
\hline & OBS & 00225 & 10.49 & 35.33 & 27.14 & & 1496.3 \\
\hline & $\begin{array}{l}\text { Sio } \\
\text { oss }\end{array}$ & $\begin{array}{l}00250 \\
00251\end{array}$ & $\begin{array}{l}09.75 \\
09.71\end{array}$ & $\begin{array}{l}35.25 \\
35.25\end{array}$ & $\begin{array}{l}27.21 \\
27.22\end{array}$ & 00.406 & $\begin{array}{l}1493.9 \\
1493.8\end{array}$ \\
\hline & 085 & 00279 & 08.96 & 35.16 & 27.27 & & 1491.3 \\
\hline & STO & 00300 & 08.23 & 35.11 & 27.34 & 00.449 & 1488.9 \\
\hline & 085 & 00303 & 08.11 & 35.10 & 27.36 & & 1480.5 \\
\hline & 085 & 00352 & 06.94 & 35.06 & 27.49 & & 1484.7 \\
\hline & DES & 00391 & 06.44 & 35.07 & 27.57 & & 1403.4 \\
\hline & oas & 00393 & 06.44 & 35.06 & 27.56 & & 1483.4 \\
\hline
\end{tabular}


NOOC SIA I ION DATA

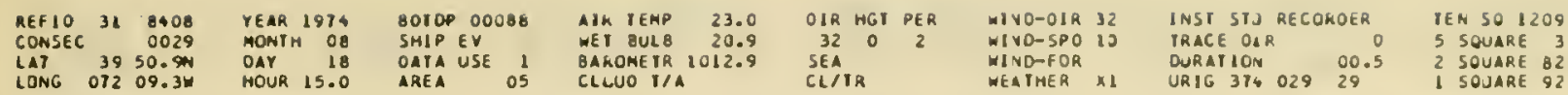

\begin{tabular}{|c|c|c|c|c|c|c|c|}
\hline \multirow[t]{2}{*}{ CASTMUMIT HE } & LVLTYP & OEPIM & IEMP & SAL & $S I S A A-T$ & OYNDPIH & SNO VEL \\
\hline & STO & 00000 & 23.66 & 36.98 & 23.75 & 00.000 & 1531.6 \\
\hline \multirow[t]{24}{*}{15.0} & 085 & 00000 & 23.86 & 34.98 & 23.75 & & 1531.6 \\
\hline & sto & 00010 & 23.06 & 35.10 & 23.84 & 00.041 & 1531.9 \\
\hline & 085 & 00011 & 23.64 & 35.12 & 23.88 & & 1531.9 \\
\hline & SIO & 00020 & 23.45 & 35.33 & 26.07 & 00.081 & 1531.8 \\
\hline & 085 & 00020 & 23.40 & 35.33 & 24.09 & & 1531.7 \\
\hline & 085 & 00024 & 23.01 & 35.31 & 24.19 & & 1530.8 \\
\hline & 085 & 00028 & 21.12 & 35.10 & 24.56 & & 1525.7 \\
\hline & STo & 00030 & 19.52 & 35.18 & 25.05 & 00.115 & 1521.5 \\
\hline & 085 & 00030 & 19.44 & 35.19 & 25.07 & & 1522.3 \\
\hline & 085 & 00033 & 17.94 & 35.41 & 25.62 & & 1517.4 \\
\hline & 085 & 00035 & 16.99 & 35.52 & 25.93 & & 1514.7 \\
\hline & 085 & 00031 & 16.70 & 35.52 & 26.00 & & 1513.9 \\
\hline & 085 & 00041 & 16.73 & 35.61 & 26.06 & & 1516.2 \\
\hline & 005 & 00045 & 16.02 & 35.46 & 20.11 & & 1511.9 \\
\hline & OBS & 00047 & 15.40 & 35.12 & 25.99 & & 1509.0 \\
\hline & Des & 00048 & 15.01 & 35.28 & 26.20 & & 1508.6 \\
\hline & sto & 00050 & 14.90 & 35.33 & 26.27 & 00.162 & 1508.3 \\
\hline & 085 & 00050 & 14.84 & 35.37 & 28.31 & & $2508 \cdot 2$ \\
\hline & OBS & 00058 & 16.91 & 35.54 & 26.41 & & 1500.9 \\
\hline & 085 & 00062 & 14.34 & 35.35 & 26.40 & & 1506.7 \\
\hline & o8s & 00065 & 24.00 & 35.45 & 26.55 & & 1505.8 \\
\hline & 510 & 00075 & 13.91 & 35.41 & 26.54 & 00.203 & 1505.6 \\
\hline & 085 & 00078 & 13.88 & 35.40 & 26.56 & & 1505.6 \\
\hline & 085 & 00080 & 13.87 & 35.40 & 26.54 & & 1505.0 \\
\hline
\end{tabular}

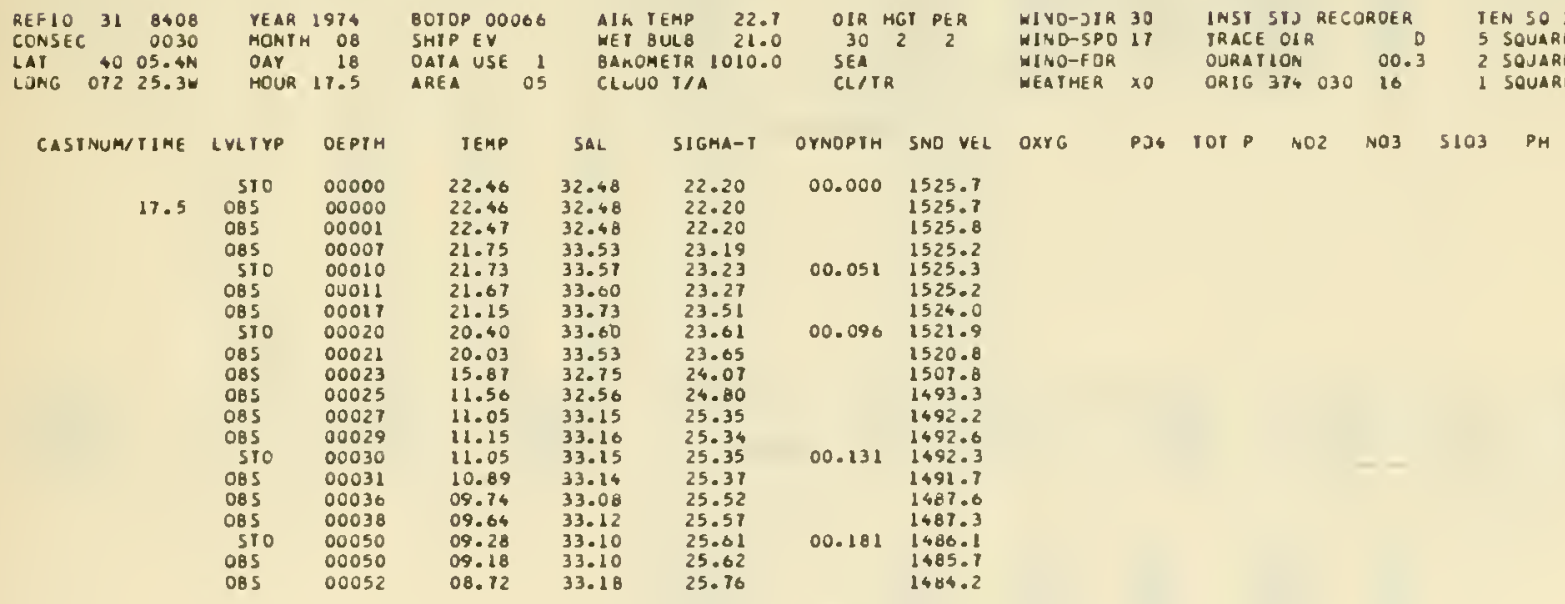




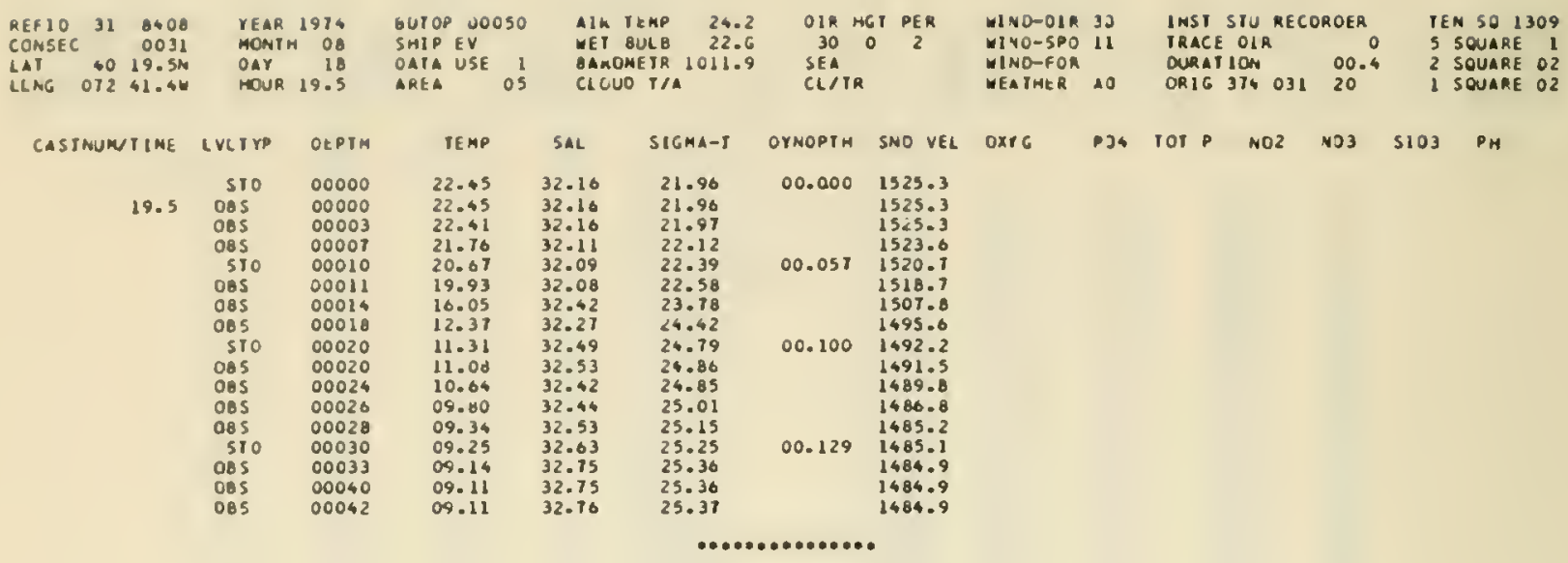

$\begin{array}{lrr}\text { REFIO } 31 & \text { O4UO } \\ \text { CONSEC } & 0032 \\ \text { LAJ } & 0 & 35.1 N \\ \text { LONG OT2 } & 59.54\end{array}$

YEAR 1974 BOTOP O0033

MONTH OB SMIPEV
OAY 16 LATA USE I

HOUR 22.2
CASTMUNIJINE IVLTYP OEPTH

\begin{tabular}{|c|c|c|}
\hline \multirow[b]{2}{*}{22.2} & STO & $\infty 0000$ \\
\hline & $\begin{array}{l}085 \\
085\end{array}$ & 00000 \\
\hline & oes & 00009 \\
\hline & 510 & 00010 \\
\hline & 085 & 00011 \\
\hline & 085 & 00013 \\
\hline & 085 & 00015 \\
\hline & 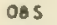 & 00019 \\
\hline & 570 & 00020 \\
\hline & OBS & 00020 \\
\hline & 085 & 00022 \\
\hline & OBS & 00024 \\
\hline & 005 & 00026 \\
\hline
\end{tabular}

$\begin{array}{ccc}\text { IEMP } & \text { SAL } & \text { SIGMA-I } \\ 22.20 & 31.18 & 21.29 \\ 22.20 & 31.18 & 21.29 \\ 22.06 & 31.21 & 21.35 \\ 20.71 & 31.19 & 21.70 \\ 20.36 & 31.24 & 21.79 \\ 19.59 & 31.22 & 22.01 \\ 18.87 & 31.36 & 22.29 \\ 18.46 & 31.43 & 22.65 \\ 17.42 & 31.56 & 22.79 \\ 16.40 & 31.59 & 23.06 \\ 15.76 & 31.66 & 23.26 \\ 15.30 & 31.92 & 23.56 \\ 15.25 & 31.95 & 23.59 \\ 15.27 & 31.97 & 23.60\end{array}$

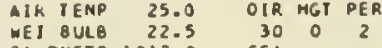
BAMONETR 1012.0 CLLUD I/A

30
SEA
CLIIR

\section{WIVD-JiR 30 INDE-SPO 3} WEATHER XO
INST STU RECOROER TEN 501309 OURATIUNA OON ORIG 37403216 I SOUARE O2
OYNOPJH SNO VEL

00.000

1523.6

1523.3

$00.003 \quad 15$

1518.9

1516.8

1514.9

1513.8

1511.0

$00.117 \quad 1507.9$

1506.1

1504.9

1504.9

OXYG PJ4 TOIP NO2 NO3 SIOJ PM

REF10 31 8408

CCNSEC 0033

LAT $40 \$ 2.7 M$
YEAR 1974
MONTH OB

OAY 19

HOUR 01.1
BOTOP O0033

SHIP EV

OATA USE
AIK TEMP 23.8 OIR HCI PER MET BUL 823.0 SAMONE IR 1010.0 $\begin{array}{ccc}\text { OIR MCT } & \text { PER } \\ 21 & 0 & 2\end{array}$ SEA $T$ TR
WIVD-OIR 21 $M I V O-5 P O \quad 12$ WINDEFOR
WEATHER XJ
INST STU RECOROER TEN SQ 1309 IRACE OIR O 5 SQUARE 1 DURATION $00.1 \quad 2$ SQUARE O2

\begin{tabular}{|c|c|c|c|c|c|c|c|}
\hline \multirow[t]{2}{*}{ CASTNUM/TIME } & LVTYP & OEPTH & TEMP & SAL & $S 1 G N A-1$ & OYNOPTH & SNO VE \\
\hline & 510 & 00000 & 21.95 & 31.31 & 21.46 & 00.000 & 1523.1 \\
\hline \multirow[t]{11}{*}{01.1} & 085 & 00000 & 21.95 & 31.31 & 21.46 & & 1523. \\
\hline & 085 & 00005 & 21.85 & 31.28 & 21.46 & & 1522. \\
\hline & 085 & 00007 & 21.30 & 31.25 & 21.59 & & 1521. \\
\hline & STO & 00010 & 21.16 & 31.28 & 21.05 & 00.063 & 1521. \\
\hline & oes & 00011 & 21.09 & 31.30 & 21.68 & & 1521. \\
\hline & 085 & 00015 & 20.91 & 31.39 & 21.80 & & 1520. \\
\hline & OBS & 00017 & 20.25 & 31.53 & 22.08 & & 1519. \\
\hline & 510 & 00020 & 19.33 & 31.74 & 22.47 & 00.120 & 1516. \\
\hline & 085 & 00020 & 19.14 & 31.78 & 22.55 & & 1516. \\
\hline & OBS & 00026 & 18.88 & 31.84 & 22.66 & & 1515 \\
\hline & o8s & 00026 & 18.65 & 31.93 & 22.74 & & 1515. \\
\hline
\end{tabular}




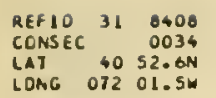

YEAR 1974
MONTH 08

MONTH 08

$\begin{array}{lr}\text { DAY } & 19 \\ \text { HOUR } 03.6\end{array}$

DOTOP 00033

SHIP EY

DATA USE

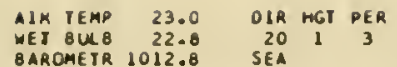

MEJ OUL 8 22.8

BAROMETR 1012.8
CLUUD T/A ino-oir 20 MIYO-SPO is $\checkmark I V O-F J R$ CLITR
SIGMA-T OYNOPIH SNO VEL OXYG

$00.000 \quad 1519.4$

21.97

23.11

$22 \cdot 11$

22.37

22.91

23.49

23. 70

24.18

24.19

24.56
1519.0

$00.057 \quad 1519.8$ 1520.0

1511.5

1506.2

1504.6

1501.4

1499.1

1695.0

1694.4
INSI STJ AECDRDER IEN SO 1300 TRACE OIR O S SOJARE ORATIOA OO.1 2 SOUARE OR DRIG 374 034

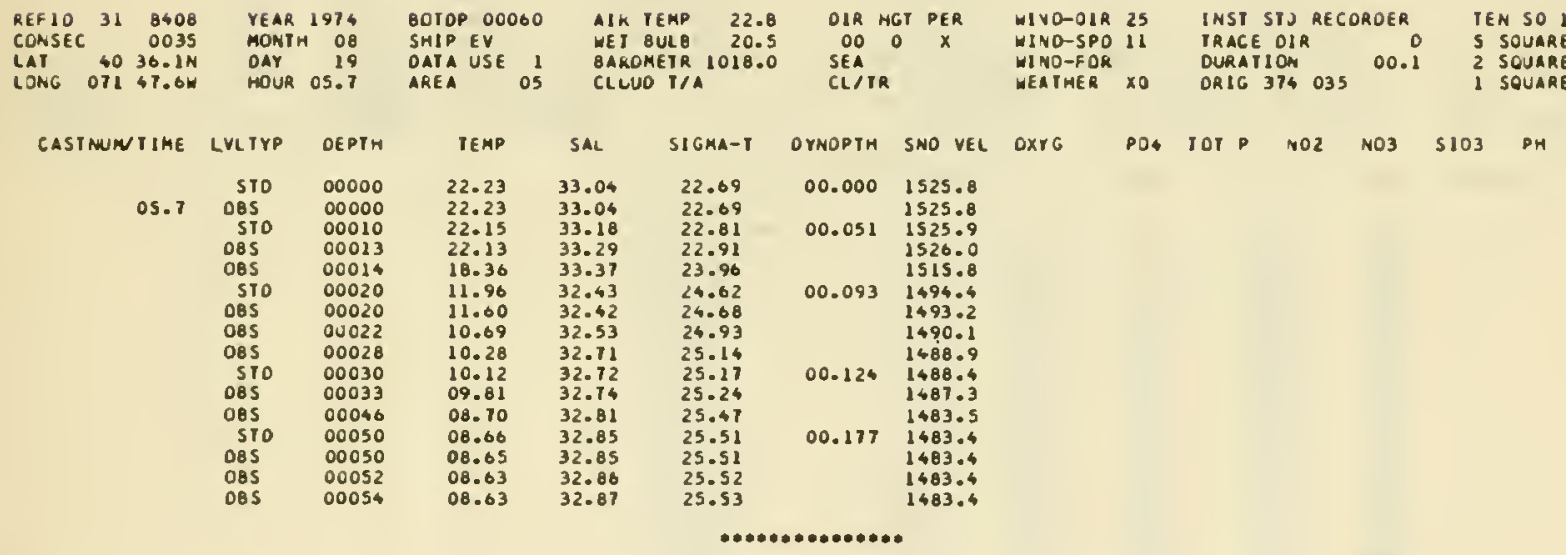


REFIO 318408 YEAF 1914 CONSEC 0030 MONIH 08

$\begin{array}{lrlll}\text { LAT } & 40 & 22.1 \mathrm{~N} & \text { OAY } 19 \\ \text { LLNG } 071 & 35.8 \mathrm{~W} & \text { MOUR } 01.9\end{array}$
OOTOP 00075

SHIP EV

OAIA USE I

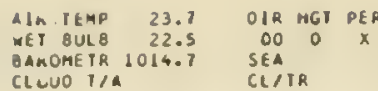

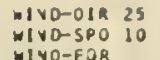

WIYO-FDR
INST SIU RECORNER

TRACE OIR DURAIION 00.1 ORIG 37. 030
IEN SO $\$ 309$ 5 SOUARE 1 1 SOUARE OI

\begin{tabular}{|c|c|c|c|}
\hline \multicolumn{2}{|c|}{ CASINUMUIINE } & L VLTYP & OEPTH \\
\hline \multirow{30}{*}{\multicolumn{2}{|c|}{07.9}} & 510 & 00000 \\
\hline & & 085 & 00000 \\
\hline & & OBS & 00001 \\
\hline & & oos & 00007 \\
\hline & & oBs & 00009 \\
\hline & & 510 & 00010 \\
\hline & & & 00011 \\
\hline & & 085 & \\
\hline & & $\begin{array}{l}\text { Oes } \\
\text { SIO }\end{array}$ & $\begin{array}{l}00018 \\
00020\end{array}$ \\
\hline & & 085 & 00020 \\
\hline & & oes & 00022 \\
\hline & & 085 & 00024 \\
\hline & & 085 & 00020 \\
\hline & & o8s & 00028 \\
\hline & & SIO & 00030 \\
\hline & & o8s & 00032 \\
\hline & & o8s & 00033 \\
\hline & & OAS & 00035 \\
\hline & & 085 & 00037 \\
\hline & & OBS & 00039 \\
\hline & & $\begin{array}{l}085 \\
085\end{array}$ & $\begin{array}{l}00043 \\
00047\end{array}$ \\
\hline & & o8s & 00048 \\
\hline & & 510 & 00050 \\
\hline & & 085 & 00050 \\
\hline & & 085 & 00052 \\
\hline & & 085 & 00054 \\
\hline & & 085 & 00050 \\
\hline & & 085 & 00058 \\
\hline & & STo & 00075 \\
\hline & & 085 & 00075 \\
\hline
\end{tabular}

$\begin{array}{lll}\text { S1GMA-1 } & \text { OYNDPIH } & \text { SND VEL } \\ & & \\ 22.59 & 00.000 & 1524.6 \\ 22.59 & & 1524.0 \\ 22.76 & & 1524.8 \\ 23.39 & & 1522.4 \\ 23.89 & & 1521.5 \\ 23.97 & 00.040 & 1521.4 \\ 24.07 & & 1521.3 \\ 23.88 & & 1522.3 \\ 24.20 & & 1517.4 \\ 24.36 & 00.084 & 1517.1 \\ 24.42 & & 1517.1 \\ 24.54 & & 1517.8 \\ 24.38 & & 1516.5 \\ 24.37 & & 1514.8 \\ 24.47 & & 1512.4 \\ 24.19 & 00.120 & 1508.5 \\ 24.50 & & 1503.5 \\ 25.31 & & 1498.3 \\ 25.30 & & 1498.3 \\ 25.25 & & 1497.1 \\ 25.43 & & 1493.7 \\ 25.70 & & 1492.4 \\ 25.83 & & 1491.5 \\ 25.81 & & 1490.8 \\ 25.75 & 00.180 & 1490.6 \\ 25.73 & & 1490.6 \\ 25.76 & & 1489.5 \\ 25.90 & & 1489.2 \\ 25.97 & & 1489.7 \\ 26.18 & & 1491.7 \\ 26.00 & 00.233 & 1494.4 \\ 26.05 & & 1494.4 \\ & & \end{array}$

$\begin{array}{lrr}\text { REF IO } & 31 & 8408 \\ \text { CONSEC } & 0037 \\ \text { LAT } & 40 & 00.0 N \\ \text { LONG OT1 } & 23.2 W\end{array}$

\section{MONTH OS} OAY 19
G010P 00089 SHIP EV OATA USE 1
AIN TEMP 22.5 OIR HGI PER

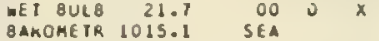
BAKOME IR 1015.1 SEA
CLLUO I/A $\operatorname{axp} 6$

P34 TOIP VO2 NO3 $\$ 133$ PH

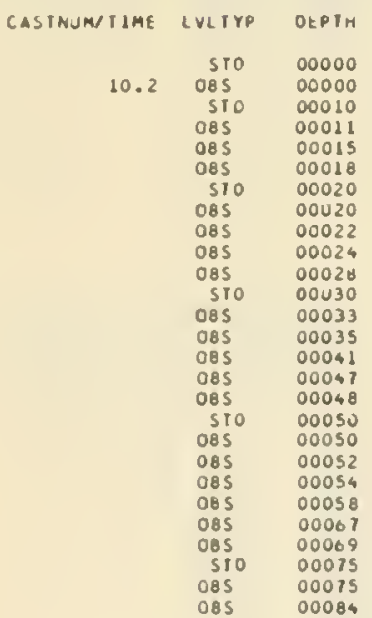

$\begin{array}{ll}\text { TEMP } & \text { SAL } \\ 21.58 & 33.86 \\ 21.58 & 33.86 \\ 21.50 & 33.97 \\ 21.49 & 33.98 \\ 21.01 & 34.55 \\ 20.68 & 34.52 \\ 20.03 & 34.52 \\ 20.47 & 34.47 \\ 19.59 & 34.18 \\ 18.69 & 34.15 \\ 17.15 & 33.98 \\ 17.22 & 33.86 \\ 13.23 & 33.57 \\ 11.06 & 33.43 \\ 09.51 & 33.54 \\ 09.28 & 33.58 \\ 09.29 & 33.74 \\ 09.46 & 33.82 \\ 09.58 & 33.88 \\ 09.88 & 33.99 \\ 10.31 & 34.04 \\ 10.40 & 34.02 \\ 10.43 & 34.30 \\ 10.19 & 34.52 \\ 11.23 & 34.56 \\ 11.24 & 34.56 \\ 11.01 & 34.59 \\ & \end{array}$

$\begin{array}{lll}\text { SISMA-I } & \text { OYNOPIH } & \text { SNO VEL } \\ & & \\ 23.49 & 00.000 & 1525.0 \\ 23.49 & & 1525.0 \\ 23.59 & 00.044 & 1525.1 \\ 23.61 & & 1525.1 \\ 24.00 & & 1526.2 \\ 24.24 & & 1523.7 \\ 24.25 & 00.084 & 1523.6 \\ 24.25 & & 1523.1 \\ 24.26 & & 1520.4 \\ 24.42 & & 1518.4 \\ 24.57 & & 1515.0 \\ 24.00 & 00.115 & 1513.3 \\ 25.14 & & 1502.4 \\ 25.56 & & 1492.8 \\ 25.91 & & 1487.4 \\ 25.98 & & 1486.7 \\ 26.11 & & 1487.0 \\ 26.15 & 00.171 & 1487.7 \\ 26.17 & & 1488.2 \\ 26.20 & & 1489.5 \\ 26.11 & & 1491.2 \\ 26.14 & & 1491.5 \\ 26.35 & & 1492.2 \\ 26.46 & & 1493.8 \\ 26.41 & 00.215 & 1495.4 \\ 20.41 & & 1495.5 \\ 20.36 & & 1497.0\end{array}$

WIVO-OIR OJ WIVO-SPO ON NTV-tjR MEATHER X4

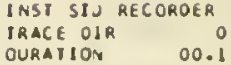
OURAIION 00.1
IEN SO 1309

S SQUARE I

2 SOUARE OO

OX8G P34 TOT YOZ Vas $\$ 133$ PH 


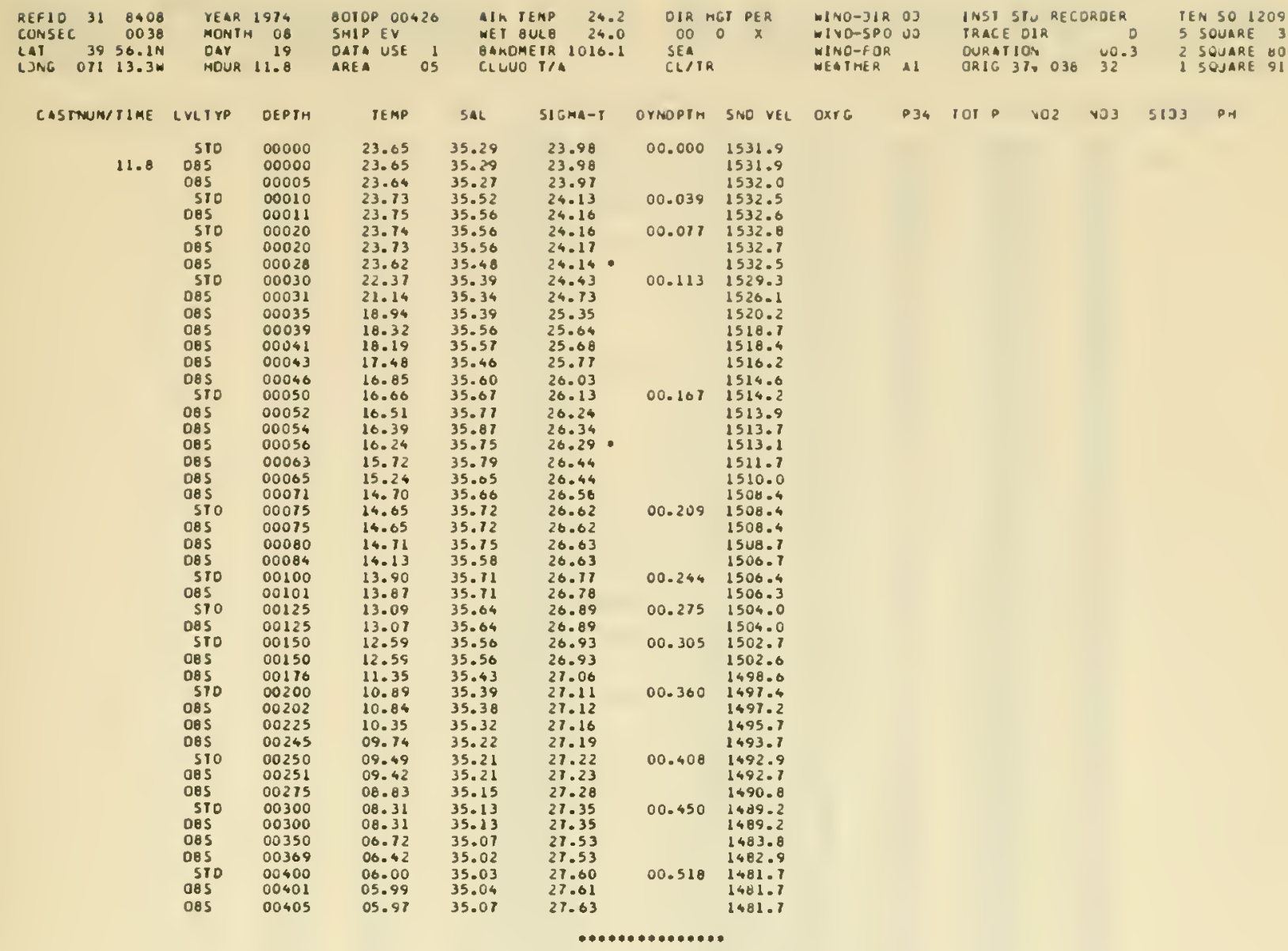




\begin{tabular}{|c|c|c|c|c|c|c|c|c|c|c|c|c|c|c|}
\hline REF 10 & 31 & 1408 & YEAR 1914 & 60108 & 02195 & AIN IEAP & 24.8 & OIR & nCl & PER & $\triangle I V D-J I R$ & 00 & INSI SIJ RECOROER & TEM SQ 1209 \\
\hline CONSEC & & 0039 & MONJ' O\& & SHID & EV & WE I OULO & 22.0 & 00 & 0 & $x$ & MIND-SPO & נט & IRACE OIR O & 5 SOUARE 3 \\
\hline GI & 39 & $41.0 \mathrm{ON}$ & oer & OATa & USE & OAROAEIR & 1010.0 & SEA & & & $M I V O-F 3 R$ & & DURA I ION & SOUARE \\
\hline LGNG & 071 & 01.14 & moua & ARE A & os & CLUNO T/ & & CL/I & & & DEATHER & 10 & ORIC 37. 039 & I SOUnine \\
\hline
\end{tabular}

\begin{tabular}{|c|c|c|c|c|c|c|c|}
\hline \multirow[t]{2}{*}{ CASIMUM IIIAF } & LVLTY & OEPIM & TEAP & SAL & $5 I G M A-I$ & OYN3PTM & SNO VEL \\
\hline & STO & 00000 & 24.05 & 35.51 & 24.03 & 00.000 & 1533.1 \\
\hline \multirow[t]{58}{*}{14.0} & $\begin{array}{l}\text { Dos } \\
\text { STO }\end{array}$ & $\begin{array}{l}00000 \\
00010\end{array}$ & $\begin{array}{l}24.05 \\
24.01\end{array}$ & $\begin{array}{l}35.51 \\
35.50\end{array}$ & $\begin{array}{l}24.03 \\
24.04\end{array}$ & 00.039 & $\begin{array}{l}1533.1 \\
1533.2\end{array}$ \\
\hline & $\begin{array}{l}005 \\
510\end{array}$ & $\begin{array}{l}00011 \\
00020\end{array}$ & $\begin{array}{l}24.00 \\
23.97\end{array}$ & $\begin{array}{l}35.50 \\
35.40\end{array}$ & $\begin{array}{l}24.04 \\
24.03\end{array}$ & 00.078 & $\begin{array}{l}1533.2 \\
1533.2\end{array}$ \\
\hline & $\begin{array}{l}085 \\
510\end{array}$ & $\begin{array}{l}00020 \\
00030\end{array}$ & $\begin{array}{l}23.90 \\
23.59\end{array}$ & $\begin{array}{l}35.48 \\
35.46\end{array}$ & $\begin{array}{l}24.04 \\
24.13\end{array}$ & 00.116 & $\begin{array}{l}1533.2 \\
2532.6\end{array}$ \\
\hline & 085 & 00010 & 23.59 & 35.46 & 24.13 & & 1532.4 \\
\hline & 005 & 00033 & 23.14 & 35.33 & 24.16 & & 1531.3 \\
\hline & 005 & 00035 & 21.39 & 35.20 & 24.56 & & 1520.7 \\
\hline & 005 & 00037 & 19.89 & 35.61 & 25.27 & & 1523.2 \\
\hline & 085 & 00039 & 19.11 & 35.43 & 25.34 & & 1520.8 \\
\hline & 005 & 00041 & 18.62 & 35.57 & 25.58 & & $1>19.6$ \\
\hline & oss & 00046 & 10.29 & 35.50 & 25.60 & & 1510.7 \\
\hline & 510 & 00050 & 17.20 & 35.37 & 25.75 & 00.177 & 1515.6 \\
\hline & Dos & 00050 & 17.15 & 35.34 & 25.70 & & 1515.2 \\
\hline & 005 & 00052 & 16.80 & 35.52 & 25.96 & & 1514.7 \\
\hline & Oes & 00056 & 16.75 & 35.55 & 26.01 & & 1514.4 \\
\hline & oss & 00001 & 16.23 & 35.69 & 26.24 & & 1513.1 \\
\hline & 085 & 00003 & 16.19 & 35.69 & $26 .+1$ & & 1513.2 \\
\hline & 005 & 00071 & 15.79 & 35.93 & 26.53 & & 1512.2 \\
\hline & 085 & 00073 & 15.08 & 35.83 & 20.40 & & 1511.7 \\
\hline & $\begin{array}{l}\text { OSS } \\
\text { STO }\end{array}$ & 00074 & 15.16 & $\begin{array}{l}35.54 \\
35.55\end{array}$ & $\begin{array}{l}26.37 \\
26.36\end{array}$ & & $\begin{array}{l}1509.8 \\
1509.7\end{array}$ \\
\hline & OBS & $\begin{array}{l}00075 \\
00070\end{array}$ & $\begin{array}{l}15.14 \\
14.82\end{array}$ & 35.72 & 26.50 & 00.226 & 1509.0 \\
\hline & OBs & 00008 & 14.69 & 35.04 & 20.70 & & 1508.9 \\
\hline & sro & 00100 & 14.64 & 35.84 & 20.16 & 00.204 & 1508.3 \\
\hline & 005 & 00101 & 14.41 & 35.84 & 26.77 & & 1508.2 \\
\hline & 510 & 00125 & 13.75 & 35.16 & 26.84 & 00.296 & $1506 \cdot 3$ \\
\hline & Oos & 00121 & 13.69 & 35.75 & 26.85 & & $1506-2$ \\
\hline & 510 & 00150 & 13.25 & 35.69 & 20.89 & 00.327 & 1505.0 \\
\hline & 085 & 00151 & 13.19 & 35.08 & 20.90 & & 1504.8 \\
\hline & ass & 00164 & 12.62 & 35.60 & 20.95 & & 1503.0 \\
\hline & 085 & 00171 & 12.37 & 35.58 & 26.99 & & 1302.4 \\
\hline & STO & 00200 & 11.05 & 35.47 & 27.04 & 00.304 & 1540.1 \\
\hline & $\begin{array}{l}\text { oes } \\
\text { oes }\end{array}$ & $\begin{array}{l}00200 \\
00217\end{array}$ & $\begin{array}{l}11.64 \\
11.28\end{array}$ & $\begin{array}{l}35.47 \\
35.42\end{array}$ & $\begin{array}{l}27.04 \\
27.07\end{array}$ & & $\begin{array}{l}1500.1 \\
1499.1\end{array}$ \\
\hline & oos & 00221 & 11.02 & 35.37 & 27.08 & & 1498.1 \\
\hline & oes & 00220 & 10.06 & 35.36 & 27.10 & & 1647.0 \\
\hline & 510 & 00250 & 10.38 & $\begin{array}{l}35.30 \\
35.29\end{array}$ & $\begin{array}{l}27.14 \\
27.15\end{array}$ & 00.436 & $\begin{array}{l}1490.3 \\
1495.9\end{array}$ \\
\hline & 085 & 00254 & $\begin{array}{l}10.27 \\
09.06\end{array}$ & $\begin{array}{l}35.29 \\
35.22\end{array}$ & 27.20 & & 1494.0 \\
\hline & Oos & 00277 & $\begin{array}{l}09.06 \\
09.21\end{array}$ & $\begin{array}{l}35.22 \\
35.18\end{array}$ & 27.24 & 00.463 & 1492.0 \\
\hline & $\begin{array}{l}\text { STO } \\
\text { OBS }\end{array}$ & & 09.14 & 35.17 & 27.25 & & 1492.4 \\
\hline & 005 & 00350 & 08.26 & 35.13 & 27.36 & & 1489.8 \\
\hline & SIO & 00400 & 07.60 & 35.09 & 27.43 & 00.565 & 1488.1 \\
\hline & 085 & 00406 & 07.50 & 35.09 & 27.44 & & 1687.8 \\
\hline & ods & 00451 & 06.15 & 35.06 & 27.52 & & 1485.6 \\
\hline & oes & $\begin{array}{l}00500 \\
00501\end{array}$ & 06.44 & 35.03 & $\begin{array}{l}27.59 \\
27.59\end{array}$ & 00.630 & 1463.5 \\
\hline & $\begin{array}{l}\text { Dos } \\
\text { Das }\end{array}$ & $\begin{array}{l}00502 \\
00552\end{array}$ & 00.02 & 35.03 & $\begin{array}{l}27.59 \\
27.05\end{array}$ & & $\begin{array}{l}1483.5 \\
1482.0\end{array}$ \\
\hline & sto & $\begin{array}{l}00552 \\
00600\end{array}$ & 05.59 & 35.03 & $\begin{array}{l}27.05 \\
27.08\end{array}$ & 00.684 & $\begin{array}{r}1482.6 \\
1482.2\end{array}$ \\
\hline & oes & $\begin{array}{l}00600 \\
00604\end{array}$ & 05.32 & 35.03 & $\begin{array}{l}27.08 \\
27.09\end{array}$ & & $\begin{array}{l}1482.2 \\
1482.2\end{array}$ \\
\hline & 085 & $\begin{array}{l}00604 \\
00651\end{array}$ & 05.29 & 35.03 & $\begin{array}{l}27.69 \\
27.71\end{array}$ & & $\begin{array}{l}1482.2 \\
1482.0\end{array}$ \\
\hline & STO & 00700 & $\begin{array}{l}05.06 \\
04.64\end{array}$ & $\begin{array}{l}35.03 \\
35.00\end{array}$ & 27.72 & 00.732 & 1481.9 \\
\hline & $\begin{array}{l}\text { Oos } \\
005\end{array}$ & $\begin{array}{l}00700 \\
00750\end{array}$ & $\begin{array}{l}04.84 \\
04.78\end{array}$ & $\begin{array}{l}35.00 \\
35.01\end{array}$ & $\begin{array}{l}27.72 \\
27.73\end{array}$ & & $\begin{array}{l}1481.9 \\
1482.5\end{array}$ \\
\hline & STO & 00000 & 04.71 & 35.01 & 27.74 & 00.779 & 1433.0 \\
\hline & 085 & 00007 & 04.69 & 35.01 & 27.76 & & 1483.1 \\
\hline & Des & 00850 & 04.51 & 35.00 & 27.75 & & 1483.0 \\
\hline & STO & 00900 & 04.44 & 35.00 & 27.76 & 00.024 & 1483.6 \\
\hline & 085 & 00902 & 04.44 & 35.00 & 27.76 & & 1483.6 \\
\hline & Dos & 00951 & 04.37 & 35.00 & 27.77 & & 1484.1 \\
\hline & 085 & 00973 & 04.33 & 34.99 & 27.70 & & 1484.3 \\
\hline & STO & 01000 & 04.30 & 34.99 & 27.77 & 00.868 & 1484.6 \\
\hline & 085 & 01000 & 04.30 & 34.99 & 27.77 & & 1484.6 \\
\hline & oes & 01086 & 04.19 & 35.00 & 27.79 & & 1485.6 \\
\hline
\end{tabular}




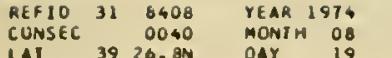
LAI $3926.8 \mathrm{~N}$ OAY 19 LLNG $07049.8 \mathrm{~W}$
BOTOP 02437 SMIP EV OATA USE
AIN TEYP 26.5 OIR HGT PER WET BUL 8 26.0 CLLUD T/A

OIR HGI PER
OO O O X
SEA
CL/TR

Nind-oir ja WIVU-SPO OO WIVU-FJR WEATHER $\angle J$
INSI SIJ AECOKOER

\section{TRACE OIR}

ORIG 374040
IEN SJ 1209

5 SOUARE 3

2 souare 0

1 SOUARE 90

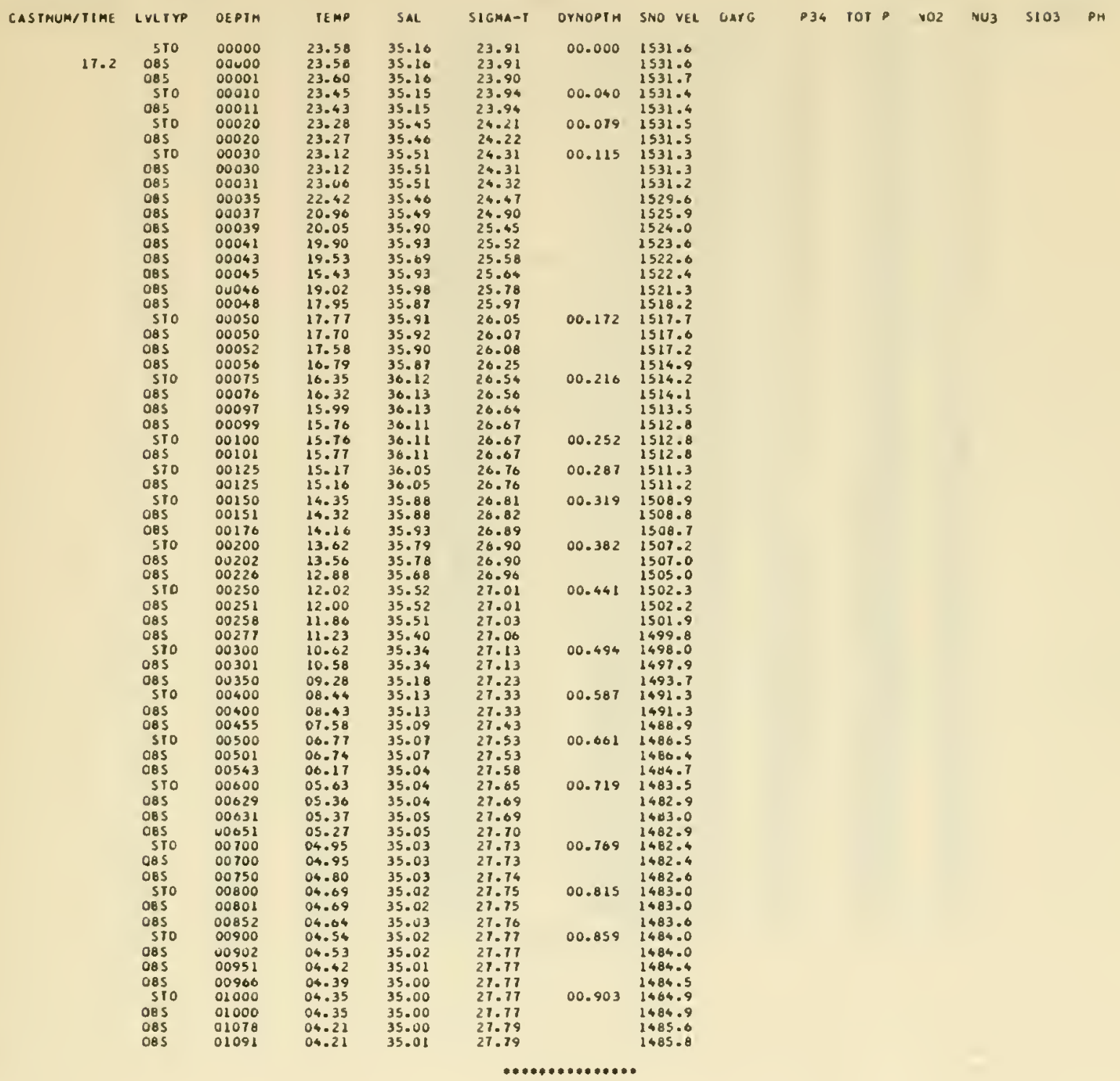




$\begin{array}{lrr}\text { REFIO } 31 & 0400 \\ \text { CONSEC } & 0041 \\ \text { LAI } & 39 & 30.5 \mathrm{M} \\ \text { LONG OTO } & 29.54\end{array}$

YEAR 1974 MONIH OA HOUR 19.8
SOIOP 02423 SHIP EY

OATA USE I
AIK IEMP 26.0 WEI BUL 825. omONETR 1010.0 BHROMETR 1018.0
OIR HGI PER $090 X$ SEA $\because V 0-0 I R \quad 18$ $M I$ I $U$ - $F O R$
INST SIU RECOROER IEN SO 1209 IRACE OIR OR S SOJARE

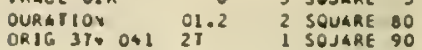

\begin{tabular}{|c|c|c|c|c|c|c|c|}
\hline \multirow[t]{2}{*}{ CASINUMA IIAEE } & LVLIYP & OEPIM & IE MP & $S A L$ & $5 J G M A-1$ & OYNDPIH & SNO VEL \\
\hline & sro & 00000 & 24.92 & 35.39 & 23.68 & 00.000 & 2535.1 \\
\hline \multirow[t]{57}{*}{19.8} & oss & 00000 & 24.92 & 35.39 & 23.68 & & $1 \$ 35.1$ \\
\hline & oos & 00001 & 24.96 & 35.39 & 23.67 & & 2535.2 \\
\hline & 005 & 00003 & 23.99 & 35.52 & 24.06 & & 1533.1 \\
\hline & sro & 00010 & 23.91 & 35.54 & $2+.10$ & 00.040 & 1533.0 \\
\hline & 085 & 00011 & 23.89 & 35.54 & 24.10 & & 2533.0 \\
\hline & s7o & 00020 & 23.70 & 35.34 & 24.16 & 00.076 & 15.72 .6 \\
\hline & OBS & 00020 & 23.68 & 35.54 & 24.16 & & 1532.6 \\
\hline & $\begin{array}{l}\text { OBS } \\
\text { STO }\end{array}$ & $\begin{array}{l}00028 \\
00030\end{array}$ & $\begin{array}{l}23.46 \\
23.08\end{array}$ & $\begin{array}{l}35.60 \\
35.66\end{array}$ & $\begin{array}{l}24.32 \\
24.43\end{array}$ & 00.115 & $\begin{array}{l}1532.3 \\
1531.4\end{array}$ \\
\hline & oes & 00030 & 23.08 & 35.66 & 24.43 & & 1531.4 \\
\hline & oes & 00033 & 22.38 & 35.73 & 24.68 & & 2529.8 \\
\hline & oes & 00035 & 21.52 & 35.79 & 24.91 & & 1527.7 \\
\hline & OBS & 00041 & 19.65 & 35.93 & 25.58 & & 1522.9 \\
\hline & OBS & 00045 & 19.10 & 35.97 & 25.75 & & 1521.5 \\
\hline & STo & 00050 & 18.28 & 35.92 & 25.93 & 00.211 & 1519.2 \\
\hline & 005 & 00050 & 18.16 & 35.91 & 25.95 & & 1518.8 \\
\hline & 085 & 00052 & 17.66 & 35.86 & 26.06 & & 1517.3 \\
\hline & OBS & 00069 & 16.44 & 35.98 & 26.42 & & 1524.2 \\
\hline & STO & 00075 & 16.24 & 36.00 & 26.48 & 00.217 & 1513.7 \\
\hline & & & 16.18 & 36.00 & 26.49 & & 1513.5 \\
\hline & OBs & 00097 & 15.85 & 30.03 & 28.59 & & 1512.9 \\
\hline & 510 & 00100 & 15.64 & 36.01 & 26.62 & 00.255 & $1512 \cdot 3$ \\
\hline & 085 & 00101 & 15.55 & 36.00 & 26.64 & & 1512.0 \\
\hline & 510 & 00125 & 16.70 & 35.87 & 26.13 & 00.291 & 1509.5 \\
\hline & oes & 00125 & 14.68 & 35.87 & 26.13 & & 1509.5 \\
\hline & s10 & 00150 & 14.09 & 35.81 & 20.81 & 00.324 & 1507.9 \\
\hline & oss & 00151 & 14.04 & 35.60 & 20.81 & & 1507.8 \\
\hline & oss & 00176 & 13.30 & 35.68 & 20.88 & & 1505.6 \\
\hline & $\$ 10$ & 00200 & 12.63 & 35.58 & 26.93 & 00.386 & 1503.6 \\
\hline & 085 & 00200 & 12.62 & 35.38 & 26.94 & & 1503.6 \\
\hline & OBS & 00226 & 11.85 & 35.49 & 27.02 & & 1501.3 \\
\hline & STO & 00250 & 21.34 & 35.42 & 27.06 & 00.442 & 1499.8 \\
\hline & 085 & 00251 & 12.31 & 35.42 & 27.06 & & 1499.7 \\
\hline & O8s & 00275 & 10.53 & 35.30 & 27.11 & & 1691.2 \\
\hline & \$TO & 00300 & 09.17 & 35.22 & 27.19 & 00.493 & 1496.8 \\
\hline & OBS & 00301 & 09.73 & 35.22 & 27.19 & & 1494.6 \\
\hline & 085 & 00350 & 08.87 & 35.13 & 27.26 & & 1492.1 \\
\hline & STO & 00400 & 08.24 & 35.11 & 27.34 & 00.501 & 1490.6 \\
\hline & 065 & 00400 & 08.23 & 35.11 & 27.35 & & 1490.5 \\
\hline & Des & 00451 & 07.28 & 35.08 & 27.46 & & 2487.7 \\
\hline & sto & 00500 & 06.52 & 35.04 & 27.54 & 00.654 & 1485.4 \\
\hline & 085 & 00500 & 06.52 & 35.06 & 27.54 & & 1485.4 \\
\hline & 085 & 00556 & 05.84 & 35.04 & 27.63 & & 1603.6 \\
\hline & sro & 00000 & 05.37 & 35.03 & 27.68 & 00.711 & 1482.5 \\
\hline & OBS & 00603 & 05.35 & 35.03 & 27.68 & & 1482.4 \\
\hline & o8s & 00051 & 05.18 & 35.03 & 27.70 & & 1482.5 \\
\hline & STO & 00700 & 04.93 & 35.02 & 27.72 & 00.759 & 1482.3 \\
\hline & obs & oorno & 04.93 & 35.02 & 27.12 & & 1682.3 \\
\hline & abs & 00756 & 06.74 & 35.01 & 21.13 & & 1482.4 \\
\hline & $\begin{array}{l}510 \\
085\end{array}$ & $\begin{array}{l}00800 \\
00805\end{array}$ & $\begin{array}{l}04.60 \\
04.65\end{array}$ & $\begin{array}{l}35.01 \\
35.01\end{array}$ & $\begin{array}{l}27.14 \\
27.14\end{array}$ & 00.805 & 1487.8 \\
\hline & OBS & 00852 & 04.62 & 35.01 & 27.75 & & $\begin{array}{l}1482.9 \\
1483.5\end{array}$ \\
\hline & 510 & 00900 & 04.51 & 35.01 & 27.76 & 00.850 & 1483.9 \\
\hline & oes & 00900 & 04.51 & 35.01 & 27.76 & & 1683.9 \\
\hline & 085 & 00955 & 04.46 & 35.01 & 27.77 & & 1484.5 \\
\hline & 510 & 01000 & 04.39 & 35.01 & 27.77 & 00.894 & 1485.0 \\
\hline & 085 & 01000 & 04.39 & 35.01 & 27.77 & & $14 a 5.0$ \\
\hline & Oas & 01018 & 04.33 & 34.99 & 21.76 & & 1485.1 \\
\hline & oes & 01091 & 04.21 & 35.01 & 27.79 & & 1485.8 \\
\hline
\end{tabular}




\begin{tabular}{|c|c|c|c|c|c|c|c|c|c|c|c|c|c|c|c|}
\hline REF 10 & 31 & 8408 & YEAR 1976 & Gor op & 01854 & AIN IEMP & 24.0 & $O \perp R$ & MGT & PER. & WINO-JIR & 00 & INSI STJ & RECDROER & TEN SO 1209 \\
\hline CUNSEC & & 0042 & MUMIN OB & SHIP & EV & WEI BULB & 21.6 & 00 & 0 & $A$ & WIVD-SPO & 00 & IRACE OIF & $R \quad 0$ & S SOUARE \\
\hline LAT & 39 & $46.0 \mathrm{~N}$ & OAY & OATA & USE & BAKOMEIR & 1017.7 & SEA & & & WIYO-FOR & & OURATION & 01.2 & SOJARE \\
\hline LJNG & 070 & $30.8 \%$ & MOUR 22.3 & ARt A & 05 & CLwUo II & & $\mathrm{Cb} / \mathrm{s}$ & & & WEHINER & $x_{0}$ & ORIG 314 & 042 & SOUARE \\
\hline
\end{tabular}

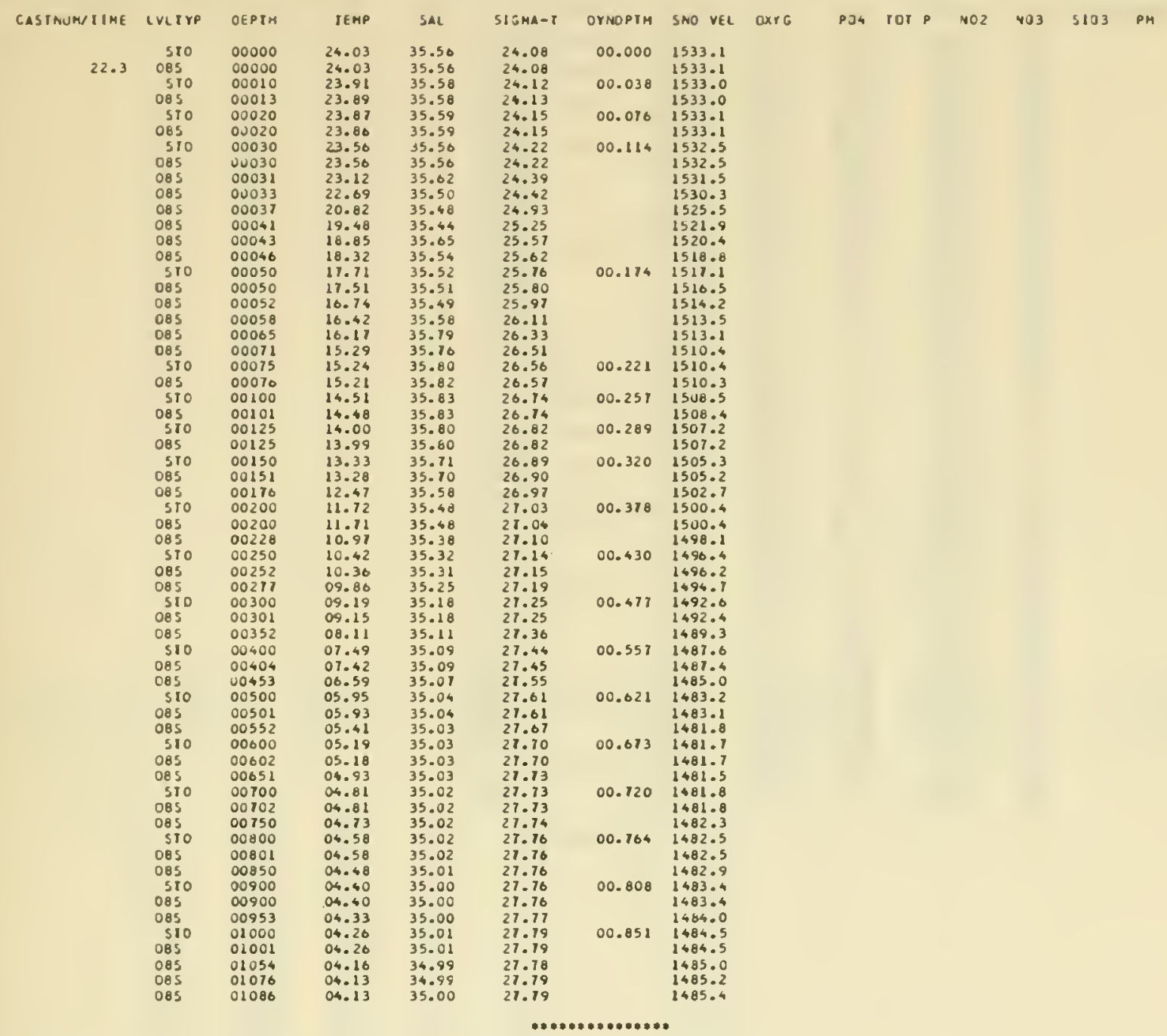




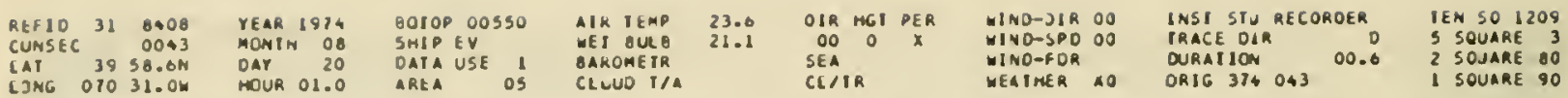

\begin{tabular}{|c|c|c|c|c|c|c|c|}
\hline \multirow[t]{2}{*}{ CASINWN I IME } & LVt Tre & DEPIN & IE MP & SAL & SIGMA-T & OYNDPTH & SNO VEL \\
\hline & 510 & $\infty 0000$ & 24.14 & 35.36 & 23.89 & 00.000 & 1533.2 \\
\hline \multirow[t]{46}{*}{01.0} & oes & $\infty 0000$ & 24.14 & 35.36 & 23.89 & & 1533.2 \\
\hline & SID & 00010 & 23.94 & 35.35 & 23.95 & 00.040 & 1532.0 \\
\hline & 085 & 00011 & 23.92 & 35.33 & 23.95 & & 1532.6 \\
\hline & SID & 00020 & 23.86 & 35.52 & 24.10 & 00.079 & 1533.0 \\
\hline & Des & 00020 & 23.85 & 35.5 .3 & 24.11 & & 1533.0 \\
\hline & 005 & & 23.64 & 35.58 & 24.21 & & 1532.7 \\
\hline & STD & 00030 & 23.53 & 35.60 & 24.25 & 00.118 & 1532.5 \\
\hline & Des & 00031 & 23.31 & 35.61 & 24.33 & & 1532.0 \\
\hline & Des & & 21.97 & 35.61 & 24.71 & & 1528.7 \\
\hline & 085 & 00039 & & $\begin{array}{l}35.60 \\
35.30\end{array}$ & $\begin{array}{l}24.94 \\
25.04\end{array}$ & & 1526.4 \\
\hline & 065 & & $\begin{array}{l}19.88 \\
10.75\end{array}$ & $\begin{array}{l}33.30 \\
35.30\end{array}$ & $\begin{array}{l}25.04 \\
25.49\end{array}$ & & 1522.0 \\
\hline & $\begin{array}{l}\text { OBS } \\
\text { S10 }\end{array}$ & $\begin{array}{l}00043 \\
00050\end{array}$ & 10.69 & 39.63 & $\begin{array}{l}23.49 \\
25.75\end{array}$ & 00.176 & $\begin{array}{l}1519.9 \\
1520.3\end{array}$ \\
\hline & oss & 00050 & $\mid 8.61$ & 35.86 & 25.78 & & 1520.3 \\
\hline & 085 & 00052 & 18.59 & 35.13 & 25.70. & & 1519.9 \\
\hline & De's & 00054 & 17.63 & 35.12 & 25.93 & & 1517.2 \\
\hline & 085 & 00056 & 17.16 & 35.72 & 26.05 & & 1515.8 \\
\hline & Dos & 00058 & 16.68 & 35.40 & 25.91. & & 1514.0 \\
\hline & DBS & 00060 & 15.67 & 35.57 & 26.28 & & 1511.2 \\
\hline & 510 & 00075 & 15.30 & 35.80 & 26.53 & 00.224 & 1510.7 \\
\hline & 085 & 00070 & 15.29 & 35.83 & 20.56 & & 1510.0 \\
\hline & $\begin{array}{l}\text { Dos } \\
\text { STO }\end{array}$ & $\begin{array}{l}00082 \\
00100\end{array}$ & $\begin{array}{l}15.04 \\
15.00\end{array}$ & $\begin{array}{l}35.78 \\
35.94\end{array}$ & $\begin{array}{l}26.58 \\
26.11\end{array}$ & 00.260 & $\begin{array}{l}1509.8 \\
1510.2\end{array}$ \\
\hline & 005 & 00101 & 14.90 & 35.94 & 20.12 & & 1510.1 \\
\hline & 510 & 00125 & 14.13 & 33.80 & 26.19 & 00.294 & 1507.7 \\
\hline & 085 & 00125 & 14.13 & 35.80 & 26.79 & & 1307.6 \\
\hline & $\begin{array}{l}\text { STO } \\
\text { OES }\end{array}$ & $\begin{array}{l}00150 \\
00150\end{array}$ & $\begin{array}{l}13.49 \\
13.49\end{array}$ & $\begin{array}{l}35.11 \\
35.11\end{array}$ & $\begin{array}{l}26.86 \\
26.86\end{array}$ & 00.325 & $\begin{array}{l}1505.8 \\
1505.8\end{array}$ \\
\hline & Des & 00176 & 12.83 & 35.64 & 26.94 & & 1504.0 \\
\hline & OBS & 00187 & 12.44 & 35.35 & 20.95 & & 1502.1 \\
\hline & Sio & 00200 & 11.73 & 35.47 & 27.03 & 00.384 & 1500.4 \\
\hline & 005 & 00200 & 11.70 & 35.47 & 21.03 & & 1500.3 \\
\hline & 085 & 00228 & 10.78 & 35.38 & 21.13 & & $1+97.4$ \\
\hline & Dos & 00234 & 10.63 & 35.35 & 21.13 & & 1496.9 \\
\hline & Sio & 00250 & 10.10 & 35.30 & 21.19 & 00.433 & 1495.2 \\
\hline & OBS & 00251 & 10.06 & 35.30 & 21.19 & & 1495.1 \\
\hline & Des & 00215 & 09.28 & 35.18 & 27.23 & & 1492.5 \\
\hline & Des & 00286 & 08.89 & 35.16 & 27.28 & & 1491.2 \\
\hline & Des & 00290 & 08.53 & 35.12 & 27.31 & & 1489.9 \\
\hline & Des & 00296 & 08.37 & 35.14 & 27.35 & & 1489.4 \\
\hline & DBS & 00299 & 06.12 & 35.11 & 27.36 & & 1486.4 \\
\hline & SID & 00300 & 08.12 & 35.11 & $27 \cdot 36$ & 00.478 & 1488.4 \\
\hline & 085 & 00301 & 08.05 & 35.13 & 27.39 & & 1488.2 \\
\hline & 085 & 00352 & 00.79 & 35.07 & 27.32 & & $1484 \cdot 1$ \\
\hline & o8s & 00391 & 06.01 & 35.04 & 27.60 & & 1481.6 \\
\hline & STO & 00400 & 03.86 & 35.05 & 27.63 & 00.543 & $14 a 1.1$ \\
\hline & OBS & 00401 & 05.84 & 35.05 & 27.63 & & 1481.1 \\
\hline & DBS & 00436 & 05.61 & 35.04 & 27.65 & & 1480.8 \\
\hline & Dos & 00447 & 05.63 & 35.07 & 27.68 & & 1481.0 \\
\hline
\end{tabular}




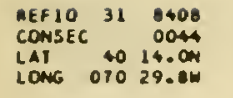

YEAR 1974

MONIH OB

$\begin{array}{lr}\text { DAY } & 20 \\ \text { HOUK } & 03.4\end{array}$
SCTOP 00106

SHIP EV

OATA USE I
AIK TEMP 23.0 OIR HGI PER

WEI BULB 21.5 BARONE TR 1010.9 CLLUO J/A

OIR HGI PER
OO O O X
SEA
CL/IR

MINDTOIR JJ WIVO-SPO 00 WINO-FOR WESIHER XO
INSI SIO RECOROER IEN SZ 1309 IRACE OIR O 5 SQUARE OUR IION 00.1

\begin{tabular}{|c|c|c|c|c|c|c|c|}
\hline CASINUM/TIME & LVLTR & OEPIN & TEMP & SAL & SIGMA-I & OYNOPIM & SNO VEL \\
\hline \multirow{27}{*}{03.4} & 510 & 00000 & 21.62 & 34.26 & 23.78 & 00.000 & 1525.6 \\
\hline & 085 & 00000 & 21.62 & 34.26 & 23.70 & & 1525.6 \\
\hline & Oos & 00001 & 21.38 & 34.24 & 23.83 & & 1525.0 \\
\hline & 085 & 00001 & 21.33 & 34.24 & 23.85 & & 1524.9 \\
\hline & STD & 00010 & 20.74 & 34.10 & 23.90 & 00.041 & $15<3.2$ \\
\hline & 065 & 00013 & 20.03 & 34.00 & 24.01 & & 1521.3 \\
\hline & 085 & 00018 & 18.90 & 36.04 & 26.33 & & 1518.2 \\
\hline & STO & 00020 & 18.29 & 34.01 & 24.51 & 00.018 & 1516.6 \\
\hline & 085 & 00022 & 17.39 & 34.08 & 24.74 & & 1516.0 \\
\hline & 085 & 00026 & 17.06 & 33.96 & 24.72 & & 1512.9 \\
\hline & 085 & 00020 & 16.06 & 33.85 & 24.87 & & 1509.8 \\
\hline & STO & 00030 & 15.34 & 33.76 & 24.96 & 00.110 & 1507.4 \\
\hline & 085 & 00032 & 16.22 & 33.64 & 25.11 & & 1503.7 \\
\hline & $\begin{array}{l}085 \\
085\end{array}$ & $\begin{array}{l}00033 \\
00039\end{array}$ & $\begin{array}{l}13.07 \\
12.60\end{array}$ & $\begin{array}{l}33.87 \\
33.73\end{array}$ & $\begin{array}{l}25.52 \\
25.51\end{array}$ & & $\begin{array}{l}1500.2 \\
1498.6\end{array}$ \\
\hline & 085 & 00041 & 11.53 & 33.60 & 25.60 & & 1496.9 \\
\hline & Dos & 00043 & 10.88 & 33.69 & 25.80 & & 1492.0 \\
\hline & 570 & 00050 & 10.56 & 33.74 & 25.89 & 00.162 & 1491.6 \\
\hline & 085 & 00050 & 10.55 & 33.75 & 25.90 & & 1491.6 \\
\hline & 085 & 00058 & 10.05 & 33.92 & 26.02 & & 1492.3 \\
\hline & oos & 00060 & 10.95 & 33.97 & 26.00 & & 1493.5 \\
\hline & 550 & 00075 & 10.56 & 33.94 & 26.05 & 00.213 & 1492.3 \\
\hline & 085 & 00075 & 10.55 & 33.94 & 26.05 & & 1492.3 \\
\hline & 085 & 00077 & 10.49 & 33.97 & 26.08 & & 1492.1 \\
\hline & 085 & 00079 & 10.49 & 33.97 & 26.08 & & 1492.1 \\
\hline & 085 & 00082 & 10.82 & 34.26 & 26.25 & & 1493.7 \\
\hline & oes & 00084 & 11.43 & 34.45 & 26.29 & & 1490.2 \\
\hline & 085 & 00092 & 11.82 & 34.46 & 26.22 . & & 1497.7 \\
\hline
\end{tabular}

$\begin{array}{lllllll}\text { OXYG } & 107 & 102 & \mathrm{~N}_{3} & 5103 & \mathrm{PH}\end{array}$

$\begin{array}{lrrl}\text { REF1O } 31 & 8408 & \text { YEAR } 197 \\ \text { CONSEC } & 0045 & \text { MONTH } \\ \text { LAT } & 00 & 35.2 \mathrm{~N} & \text { DAY } \\ \text { LUNG 010 } & 30.0 \mathrm{~W} & \text { HOUR OS. }\end{array}$

CASINUNIIHE LVLTYP OEPIH

\begin{tabular}{|c|c|c|}
\hline \multirow{19}{*}{05.7} & S10 & 00000 \\
\hline & 085 & 00000 \\
\hline & OBS & 00003 \\
\hline & S10 & 00010 \\
\hline & OBS & 00015 \\
\hline & 085 & 00018 \\
\hline & STO & 00020 \\
\hline & 085 & 00020 \\
\hline & OBS & 00024 \\
\hline & 085 & 00028 \\
\hline & 510 & 00030 \\
\hline & OBS & 00031 \\
\hline & 085 & 00035 \\
\hline & 085 & 00037 \\
\hline & OBS & 00041 \\
\hline & 085 & 00043 \\
\hline & 510 & 00050 \\
\hline & 085 & 00052 \\
\hline & OBS & 00059 \\
\hline
\end{tabular}

BOTOP 00060

SHIP EV

ORIA USE 1

AIR IEMP 23.2 OIA HGT PEA

WET BULB 21.B $000 X$

BAROMETR 1019.3

CLLUD T/A

SEA $C L / T A$

MINO-JIR DO

WINDESPD DO

WINO-FJR

WEA IHER XO
INSI STU RECOROER IEN SO 1309 TRACE DIR O 5 SOUARE 1 OURATION OO.1 2 SQUARE OO

\begin{tabular}{|c|c|c|c|c|c|c|c|c|c|c|c|}
\hline TEMP & SAL & SIGMA-I & D YNOPIH & SNO VEL & oxre & PJ4 & TOT & $p$ & 102 & $\mathrm{NO} 3$ & 5103 \\
\hline 21.92 & 34.01 & 23.51 & 00.000 & 1526.1 & & & & & & & \\
\hline 21.92 & 34.01 & 23.51 & & 1520.1 & & & & & & & \\
\hline 21.81 & 34.34 & 23.79 & & 1526.2 & & & & & & & \\
\hline 21.60 & 34.48 & 23.95 & 00.042 & 1526.0 & & & & & & & \\
\hline 21.44 & 34.59 & 24.08 & & 1525.8 & & & & & & & \\
\hline 20.69 & 34.35 & 24.10 & & 1523.5 & & & & & & & \\
\hline 20.53 & 34.11 & 23.96 & 00.081 & 1522.9 & & & & & & & \\
\hline 20.36 & 34.01 & 23.93 & & 1522.3 & & & & & & & \\
\hline 17.98 & 33.61 & $\begin{array}{l}24.23 \\
24.58\end{array}$ & & $\begin{array}{l}1515.2 \\
1511.4\end{array}$ & & & & & & & \\
\hline 15.83 & 33.47 & & 00.118 & 1508.6 & & & & & & & \\
\hline 14.67 & 33.34 & 24.78 & & 1504.8 & & & & & & & \\
\hline 11.67 & 33.20 & 25.31 & & 1493.9 & & & & & & & \\
\hline 11.51 & 33.50 & 25.53 & & 1494.7 & & & & & & & \\
\hline 10.47 & 33.11 & $25.42 *$ & & 1490.3 & & & & & & & \\
\hline 10.04 & 33.27 & 25.62 & & 1489.0 & & & & & & & \\
\hline 09.82 & 33.31 & 25.69 & 00.174 & 1468.4 & & & & & & & \\
\hline 09.79 & 33.33 & 25.71 & & 1488.3 & & & & & & & \\
\hline 09.84 & 33.38 & 25.74 & & 1488.7 & & & & & & & \\
\hline
\end{tabular}



CONSEC OO46 MONIM OB LAI $40 \quad 53.4 \mathrm{H}$ LCNG U70 $31.9 \%$ 4 (n) MOUR OY.
BUTOP 00049

SMIP EV

OAIA USE I

in IEM

IIN TENP 25.4

GAKONETA 1017.5

CLLUO T/A

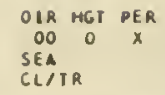

SEA 0 X $X$

CLITR
WINO-OIR DO

$M i N D=250$ OJ

WINO-F JR

WEATHER DO
INSI STJ RECOROER IEN SO 1309 TRACE OIR O S SQUARE OURATIUH OO.2 2 SQUARE OO ORIG 374 Q46 OO.L I SOUARE OO

\begin{tabular}{|c|c|c|c|c|c|c|c|}
\hline \multirow[t]{2}{*}{ CASTNUA/TIME } & LVETYP & DEPIM & IEMP & SAL & 5 IGMA-T & OYNOPTH & SNO VEC \\
\hline & S10 & 00000 & 20.17 & 31.94 & 22.41 & 00.000 & 1519.0 \\
\hline \multirow[t]{18}{*}{07.8} & Des & 00000 & 20.17 & 31.94 & 22.41 & & 1519.0 \\
\hline & OAS & 00001 & 19.59 & 31.91 & 22.54 & & 1517.4 \\
\hline & Des & 00003 & 28.00 & 31.89 & 22.92 & & 1512.9 \\
\hline & 085 & 00005 & 15.51 & 32.38 & 23.87 & & 1505.9 \\
\hline & DBS & 00009 & 15.24 & 32.42 & 23.96 & & 1505.1 \\
\hline & STO & 00010 & 14.87 & 32.43 & 24.04 & 00.047 & 2504.0 \\
\hline & D65 & 00011 & 14.23 & 32.44 & 24.19 & & 1501.9 \\
\hline & DBS & 00013 & 13.26 & 32.46 & 24.40 & & 1496.8 \\
\hline & oos & 00014 & 12.87 & 32.60 & 24.58 & & 1497.7 \\
\hline & oes & 00016 & 12.84 & 32.59 & 24.58 & & 1497.6 \\
\hline & OES & 00018 & 12.49 & 32.56 & 24.62 & & 1496.4 \\
\hline & S10 & 00020 & 12.36 & 32.64 & 24.71 & 00.082 & 1690.1 \\
\hline & OBS & 00020 & 12.34 & 32.66 & 24.73 & & 1496.0 \\
\hline & OBS & 00024 & 12.09 & 32.90 & 24.85 & & 1497.6 \\
\hline & OBS & 00028 & 12.20 & 32.90 & 26.94 & & 1496.0 \\
\hline & 085 & 00029 & 11.51 & 32.79 & 24.99 & & 1493.5 \\
\hline & $\begin{array}{l}\text { STO } \\
\text { OBS }\end{array}$ & $\begin{array}{l}00030 \\
00033\end{array}$ & $\begin{array}{l}11.48 \\
11.21\end{array}$ & $\begin{array}{l}32.79 \\
32.86\end{array}$ & $\begin{array}{l}24.99 \\
25.11\end{array}$ & 00.113 & $\begin{array}{l}1493.4 \\
1492.2\end{array}$ \\
\hline & DBS & 00044 & $11.0 i$ & 32.89 & 25.15 & & 1492.1 \\
\hline
\end{tabular}

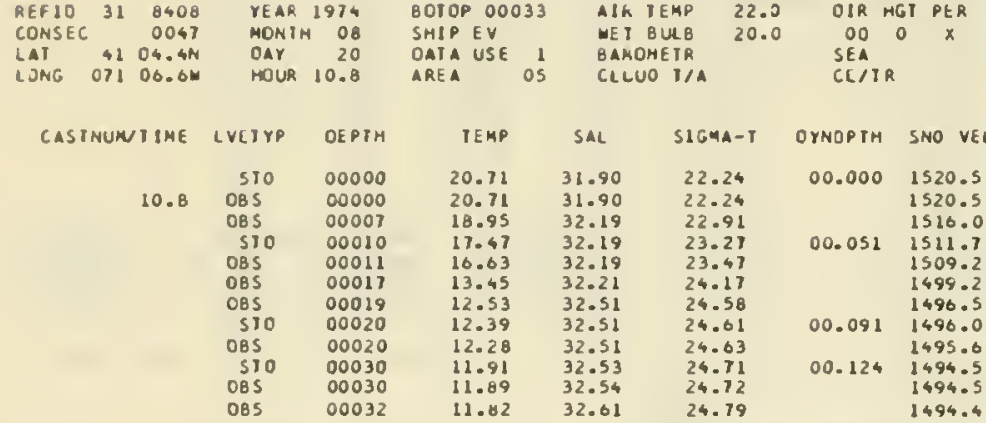

OXYG PJ4 IOI P NO2 NO3 S1O3 PH

$\ldots \ldots \ldots \cdots \cdots \cdots \cdots \cdots$

492.1

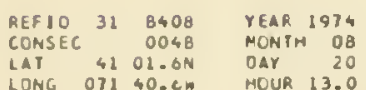

LAT $4201.0 \mathrm{~N}$

MOUR 13.0
BOIOP OOO42
SMIP EV
OAIA USE I
AREA OS

IIM IEMP

WEJ BUL 6

AREA BAKONETK 1023.0

\section{DIR MGI PEK WINO-OIR $2 T$ $000 \times$ WIVDSPO 02}

SEM WINO-FOR
INST STJ RECOROER TEN SO 1309

IRACE OIR O S SOUARE I DURATION OO.1 2 SOJARE OO ORIG 37404720 I SOUARE 11

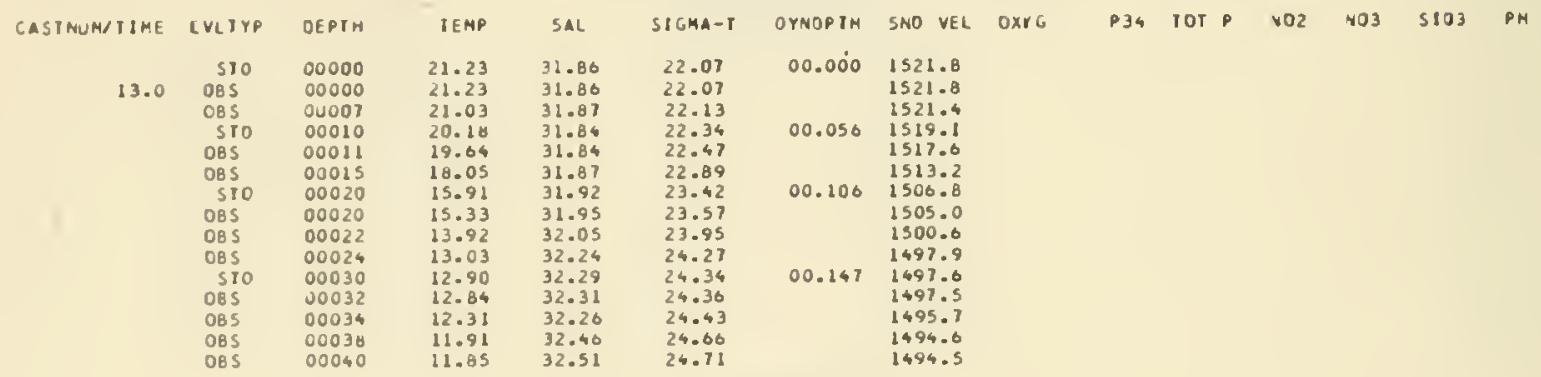

INST STU RECOROER TEN SO 1309 TRACE OIR O S SOUARE I

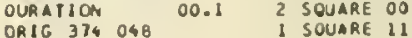




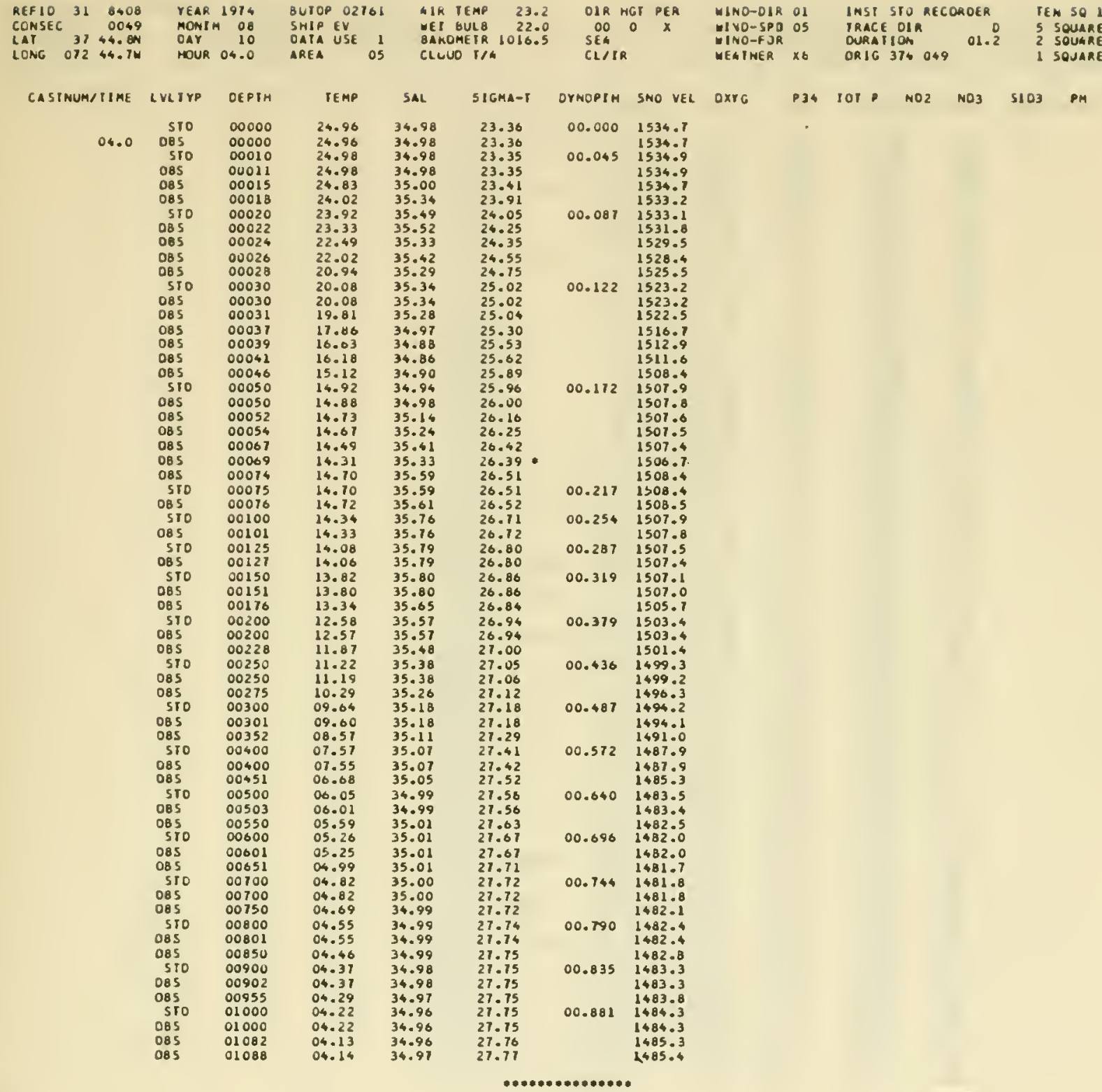


NOOC SIAIION OAIA

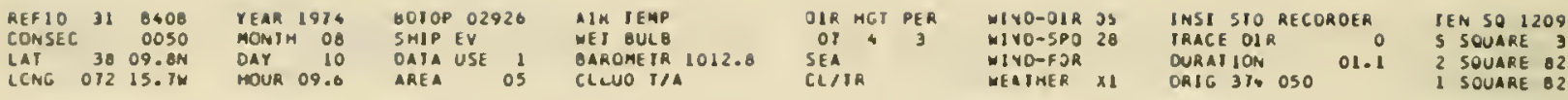

\begin{tabular}{|c|c|c|c|c|c|c|c|c|c|c|c|c|c|c|c|}
\hline \multirow[t]{2}{*}{ CASIMUNITIME } & LVLIYP & OEPTM & TEMP & SAL & SIGMA-1 & OYHOPIH & SNO VEL & $\operatorname{axy} 6$ & P36 & IOI & $p$ & $\checkmark 02$ & $\mathrm{NO}_{3}$ & 5103 & PH \\
\hline & 510 & 00000 & 24.46 & 33.66 & 22.51 & 00.000 & 1532.1 & & & & & & & & \\
\hline \multirow[t]{62}{*}{09.6} & 085 & 00000 & 26.668 & 33.60 & 22.51 & & 1532.1 & & & & & & & & \\
\hline & oes & 00008 & 24.36 & 36.73 & 23.36 & & 1533.1 & & & & & & & & \\
\hline & 085 & 00008 & 23.99 & 35.36 & 23.96 & & 1533.0 & & & & & & & & \\
\hline & 570 & 00010 & 23.93 & 35.35 & 23.95 & 00.047 & 1532.6 & & & & & & & & \\
\hline & 085 & 00011 & 23.78 & 35.36 & 23.90 & & 1532.5 & & & & & & & & \\
\hline & 085 & 00015 & 22.99 & 35.33 & 24.21 & & 1530.0 & & & & & & & & \\
\hline & 085 & 00010 & 22.10 & 35.16 & 24.33 & & 1528.2 & & & & & & & & \\
\hline & 085 & 00018 & 20.82 & 35.14 & 26.67 & & 1524.8 & & & & & & & & \\
\hline & STO & 00020 & 19.99 & 34.97 & 24.76 & 00.082 & 1322.4 & & & & & & & & \\
\hline & oBs & 00020 & 19.01 & 34.91 & 24.81 & & 1521.3 & & & & & & & & \\
\hline & 085 & 00022 & 18.49 & 36.82 & 25.03 & & 1518.1 & & & & & & & & \\
\hline & $\begin{array}{l}085 \\
085\end{array}$ & $\begin{array}{l}00026 \\
00028\end{array}$ & $\begin{array}{l}17.81 \\
16.78\end{array}$ & $\begin{array}{l}36.90 \\
36.64\end{array}$ & $\begin{array}{l}25.26 \\
25.31\end{array}$ & & $\begin{array}{l}1516.2 \\
1512.9\end{array}$ & & & & & & & & \\
\hline & 570 & 00030 & 15.22 & 36.69 & 25.70 & 00.110 & 1508.2 & & & & & & & & \\
\hline & OBS & 00030 & 15.22 & 34.09 & 25.70 & & 1508.2 & & & & & & & & \\
\hline & 085 & 00033 & 15.03 & 34.64 & 25.71 & & 1507.6 & & & & & & & & \\
\hline & $\begin{array}{l}085 \\
085\end{array}$ & $\begin{array}{l}00035 \\
00037\end{array}$ & $\begin{array}{l}16.56 \\
16.38\end{array}$ & $\begin{array}{l}34.58 \\
34.78\end{array}$ & $\begin{array}{l}25.76 \\
25.96\end{array}$ & & $\begin{array}{l}1506.1 \\
1505.8\end{array}$ & & & & & & & & \\
\hline & OBS & 00039 & 14.36 & 34.62 & 25.64 & & 1505.5 & & & & & & & & \\
\hline & 085 & 00045 & 13.13 & 34.85 & 26.15 & & 1503.8 & & & & & & & & \\
\hline & Sro & 00050 & 13.79 & 34.94 & 26.20 & 00.151 & 1504.2 & & & & & & & & \\
\hline & 005 & 00050 & 13.80 & 36.95 & 20.21 & & 1506.3 & & & & & & & & \\
\hline & $\begin{array}{l}510 \\
085\end{array}$ & $\begin{array}{l}00075 \\
00076\end{array}$ & 14.27 & 35.53 & 20.56 & 00.193 & 1500.9 & & & & & & & & \\
\hline & 085 & 00093 & 13.86 & $\begin{array}{l}35.56 \\
35.71\end{array}$ & $\begin{array}{l}26.57 \\
26.78\end{array}$ & & $\begin{array}{l}1507.1 \\
1506.1\end{array}$ & & & & & & & & \\
\hline & 510 & 00100 & 13.83 & 35.73 & 26.81 & 00.228 & 1506.2 & & & & & & & & \\
\hline & oos & 00101 & 13.83 & 35.74 & 26.81 & & 1506.2 & & & & & & & & \\
\hline & 085 & 00103 & 13.71 & 35.75 & 26.85 & & 1505.8 & & & & & & & & \\
\hline & 085 & 00106 & 13.70 & 35.74 & 26.84 & & 1505.9 & & & & & & & & \\
\hline & $\$ 10$ & 00125 & 13.09 & 35.65 & 26.89 & 00.259 & 1504.0 & & & & & & & & \\
\hline & 085 & 00127 & 13.04 & 35.64 & 26.90 & & 1503.9 & & & & & & & & \\
\hline & STo & 00150 & 12.84 & 35.62 & 26.92 & 00.289 & 1503.6 & & & & & & & & \\
\hline & OBS & 00151 & 12.82 & 35.62 & 26.93 & & 1503.5 & & & & & & & & \\
\hline & 085 & 00176 & $12+46$ & 35.59 & 26.98 & & 1502.7 & & & & & & & & \\
\hline & 510 & 00200 & 11.51 & 35.45 & 27.05 & 00.345 & 1499.0 & & & & & & & & \\
\hline & 085 & 00200 & 11.49 & 35.45 & 27.05 & & 1499.0 & & & & & & & & \\
\hline & 085 & 00226 & 10.20 & 35.26 & 27.14 & & 1695.2 & & & & & & & & \\
\hline & STO & 00250 & 09.59 & 35.22 & 27.21 & 00.395 & 1493.3 & & & & & & & & \\
\hline & 085 & 00250 & 09.57 & 35.22 & 27.22 & & 1493.2 & & & & & & & & \\
\hline & 085 & 00275 & 08.92 & 35.11 & 27.20 & & 1491.1 & & & & & & & & \\
\hline & $\$ 10$ & 00300 & 08.35 & 35.12 & 27.34 & 00.438 & 1484.3 & & & & & & & & \\
\hline & 065 & 00301 & 08.32 & 35.12 & 27.34 & & 1429.2 & & & & & & & & \\
\hline & oss & 00352 & 07.38 & 35.09 & 27.46 & & 1480.6 & & & & & & & & \\
\hline & S10 & 00400 & 06.53 & 35.06 & 27.55 & 00.508 & 1683.8 & & & & & & & & \\
\hline & 085 & 00402 & 06.69 & 35.06 & 27.56 & & 1483.1 & & & & & & & & \\
\hline & 085 & 00453 & 05.88 & 35.05 & 27.63 & & $1+82.1$ & & & & & & & & \\
\hline & sro & 00500 & 05.49 & 35.04 & 27.67 & 00.564 & 1481.3 & & & & & & & & \\
\hline & oes & 00501 & 05.40 & 35.04 & 27.67 & & 1481.3 & & & & & & & & \\
\hline & oes & 00552 & 05.18 & 35.04 & 27.11 & & 1400.9 & & & & & & & & \\
\hline & 510 & 00600 & 04.89 & 35.03 & 27.73 & 00.011 & 1480.5 & & & & & & & & \\
\hline & 085 & 00601 & 04.89 & 35.03 & 27.73 & & 1480.5 & & & & & & & & \\
\hline & 085 & 00651 & 04.75 & 35.03 & 27.75 & & 1480.8 & & & & & & & & \\
\hline & 570 & 00800 & 04.66 & 35.01 & 27.74 & 00.055 & 1481.2 & & & & & & & & \\
\hline & 085 & 00102 & 04.66 & 35.01 & 27.14 & & 1481.2 & & & & & & & & \\
\hline & 085 & 00752 & 04.55 & 35.02 & 27.76 & & 1681.6 & & & & & & & & \\
\hline & STO & 00800 & 04.46 & 35.02 & 27.78 & $00.69 \mathrm{x}$ & 1481.9 & & & & & & & & \\
\hline & 085 & 00801 & 04.46 & 35.02 & 27.78 & & 1482.0 & & & & & & & & \\
\hline & 085 & 00850 & 04.33 & 35.00 & 27.77 & & 1682.3 & & & & & & & & \\
\hline & 510 & 00900 & 04.26 & 35.00 & 27.18 & 00.760 & 1482.8 & & & & & & & & \\
\hline & 085 & 00902 & 04.26 & 35.00 & 27.18 & & $1482+9$ & & & & & & & & \\
\hline & 085 & 00953 & 04.22 & 35.00 & 27.78 & & 1483.5 & & & & & & & & \\
\hline & STO & 01000 & 04.18 & 35.00 & 27.19 & 00.182 & 1484.2 & & & & & & & & \\
\hline & oss & 01001 & 04.18 & 35.00 & 27.79 & & 1684.2 & & & & & & & & \\
\hline & oss & 01082 & 04.10 & 35.00 & 27.80 & & 1465.2 & & & & & & & & \\
\hline & Oos & 01086 & 04.10 & 35.00 & 27.80 & & 1485.2 & & & & & & & & \\
\hline
\end{tabular}




$\begin{array}{lrrl}\text { REFIO } 31 & 0408 & \text { YEAR 1974 } \\ \text { CONSEC } & 0051 & \text { MONIH OO } \\ \text { LAT } 34 & 20.0 N & \text { OAY } 10 \\ \text { LONG 072 } 33.5 W & \text { HOUR 12.4 }\end{array}$

CASTMUMATIME LVLTYP OEPTH

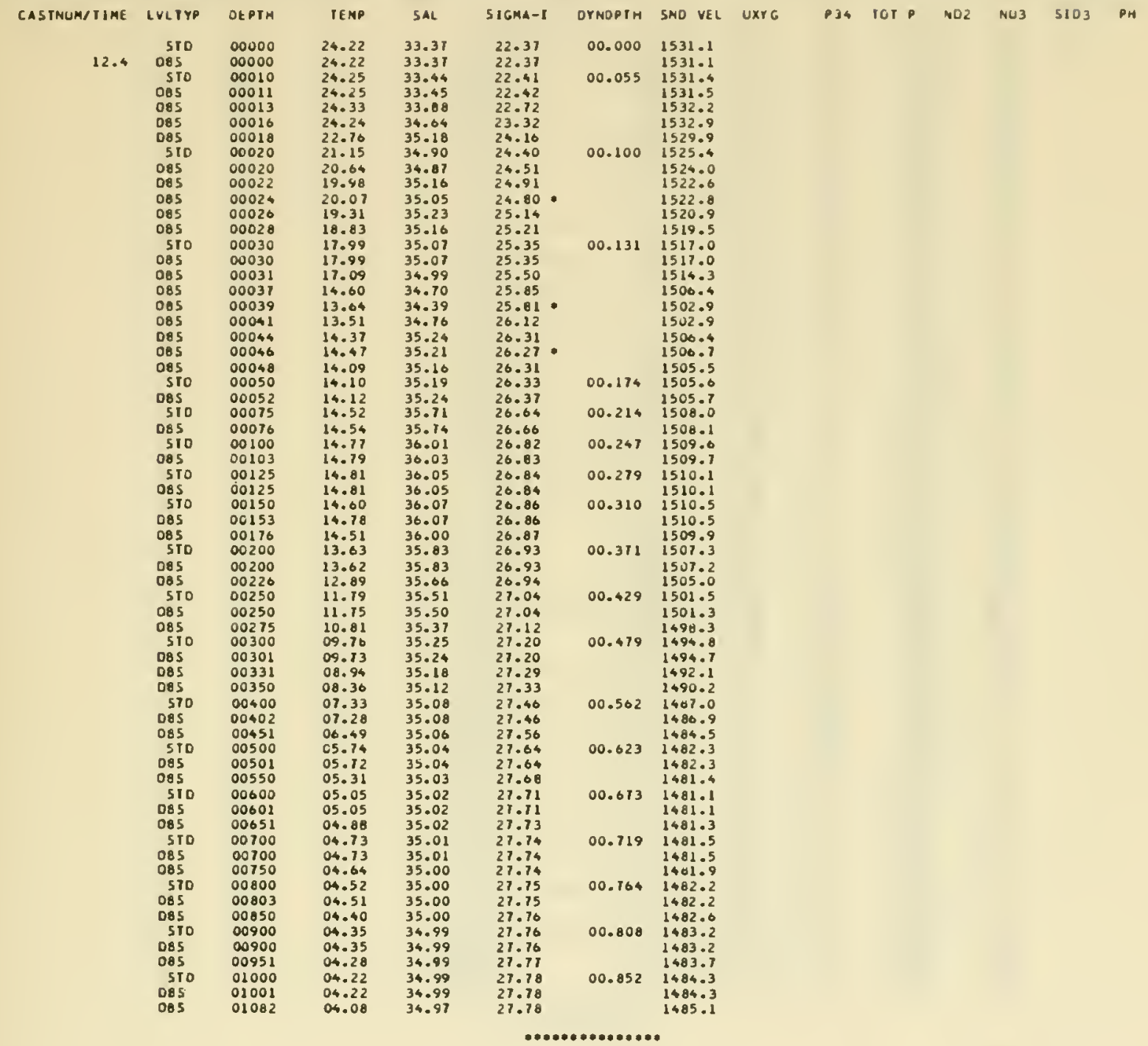

$8010 P 02743$

OAIA USE I OAKDAETR 1016.2

AREA OS

OAKDNETR 1016.2

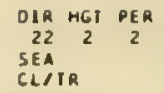

INST SIU RECOROEN IEN 501209 IRACE DLR D D SOJARE 3 OURAIION 01.2 S SOUARE 02 


$\begin{array}{lrr}\text { REF IO } 31 & 0408 \\ \text { CONSEC } & 0052 \\ \text { LAT } & 36 & 09.7 N \\ \text { LONG OT2 } & 51.34\end{array}$

YEAR 1974 BOTOP 02560 MONTH OB MOUR 19.5
CATA USE

AREA OS $\begin{array}{llc}\text { AIN TEAP } & 22.9 & \text { OIR HEI PER } \\ \text { WET EULB } & 22.2 & 03 \text { B }\end{array}$ MET BUL $8 \quad 22.2$ oamometr lo17.3 CLLUD T/A SEA
MIVO-0IR 02 WIVD-5PO 26 WIND-FOR WEATHER $x$
INST STU RECOROER TEN 501209 TRACE DIR 0 S SQUARE 3 01.2 SOUARE 82 OR1G 374052

1 SUjaRe 2

\begin{tabular}{|c|c|c|c|c|c|c|c|}
\hline CASTMUMT IME & LVLTYP & OEPIH & IEMP & SAL & $516 M A-1$ & OYNOPIH & SNO VEL \\
\hline \multirow{64}{*}{19.5} & 570 & 00000 & 23.24 & 35.55 & 24.30 & 00.000 & 1531.2 \\
\hline & 005 & 00000 & 23.24 & 35.55 & 24.30 & & 1531.2 \\
\hline & 510 & 00010 & 23.23 & 35.55 & 24.30 & 00.036 & 1531.3 \\
\hline & 005 & 00011 & 23.23 & 35.55 & 24.30 & & 1531.4 \\
\hline & 085 & 00016 & 23.08 & 35.58 & 24.37 & & 1531.1 \\
\hline & 570 & 00020 & 19.07 & 35.78 & 25.62 & 00.066 & 1520.0 \\
\hline & 085 & $\begin{array}{l}00020 \\
00028\end{array}$ & 18.51 & 35.81 & 25.78 & & 1510.3 \\
\hline & 085 & 00028 & 16.83 & 35.98 & 26.32 & & 1514.7 \\
\hline & $5 T 0$ & 00030 & 16.20 & 36.07 & 26.52 & 00.086 & 1513.2 \\
\hline & 085 & 00030 & 16.28 & 36.07 & 20.52 & & 1513.2 \\
\hline & 085 & 00031 & 15.92 & 35.90 & 26.40 & & 1511.9 \\
\hline & 085 & 00033 & 15.59 & 36.03 & 26.65 & & 1511.1 \\
\hline & oes & 00035 & 15.64 & 36.00 & 26.62 & & 1541.2 \\
\hline & 570 & 00050 & 15.16 & 35.99 & 26.72 & 00.115 & 1509.9 \\
\hline & 085 & 00052 & 15.11 & 35.99 & 26.73 & & 1509.8 \\
\hline & S10 & 00075 & 15.01 & 36.09 & 26.82 & 00.147 & 1510.0 \\
\hline & 085 & 00076 & 15.01 & 36.09 & 26.83 & & 1510.0 \\
\hline & 570 & 00100 & 15.00 & 30.11 & 26.84 & 00.179 & 1510.4 \\
\hline & 085 & 00101 & 15.00 & 36.11 & 26.84 & & 1510.4 \\
\hline & STO & 00125 & 15.06 & 36.12 & 26.84 & 00.210 & 1511.0 \\
\hline & 005 & 00125 & 15.06 & 36.12 & 26.84 & & 1511.0 \\
\hline & 570 & 00150 & 15.08 & 30.13 & 26.84 & 00.242 & $1511 \cdot 5$ \\
\hline & oes & 00151 & 15.00 & 36.13 & 26.84 & & 1511.5 \\
\hline & 085 & 00176 & 15.11 & 36.14 & 26.84 & & 1312.0 \\
\hline & STO & 00200 & 15.15 & 36.15 & 26.84 & 00.305 & 1512.6 \\
\hline & 085 & 00200 & 15.15 & 36.15 & 26.84 & & 1512.6 \\
\hline & 005 & 00226 & 15.17 & 36.16 & 26.85 & & 1513.1 \\
\hline & STo & 00250 & 15.19 & 36.17 & 26.85 & 00.369 & 1513.5 \\
\hline & 085 & 00252 & 15.19 & 36.17 & 26.85 & & 1513.6 \\
\hline & 085 & 00275 & 15.15 & 36.16 & 26.85 & & 2513.8 \\
\hline & 570 & 00300 & 15.15 & 36.15 & 26.84 & 00.634 & 1514.2 \\
\hline & 065 & 00301 & 15.15 & 36.15 & 26.84 & & 1516.2 \\
\hline & 085 & 00352 & 15.16 & 36.16 & 20.85 & & 1515.1 \\
\hline & 005 & 00357 & 15.16 & 36.16 & 26.85 & & 1515.2 \\
\hline & 085 & 00380 & 13.99 & 35.89 & 26.89 & & 1311.5 \\
\hline & oes & 00383 & 13.44 & 35.14 & 26.89 & & 1509.6 \\
\hline & 085 & 00387 & 13.26 & 35.69 & 26.89 & & 1509.0 \\
\hline & 085 & 00395 & 12.27 & 35.59 & 27.01 & & 1505.6 \\
\hline & STO & 00400 & 12.12 & 35.56 & 27.02 & 00.557 & 1505.1 \\
\hline & 085 & 00400 & 12.08 & 35.56 & 27.03 & & 1505.0 \\
\hline & 085 & 00402 & 11.99 & 35.57 & 27.05 & & 1504.8 \\
\hline & 085 & 00455 & 09.92 & 35.26 & 27.19 & & 1497.9 \\
\hline & 085 & 00666 & 09.52 & 35.21 & 27.22 & & 1496.6 \\
\hline & OBS & 00471 & 09.26 & 35.19 & 27.24 & & 1495.7 \\
\hline & STO & 00500 & 08.27 & 35.12 & 27.35 & 00.656 & 1492.3 \\
\hline & 085 & 00503 & 08.15 & 35.11 & 27.36 & & 1491.9 \\
\hline & 085 & 00550 & 07.04 & 35.07 & 27.49 & & 1486.3 \\
\hline & 570 & 00600 & 00.13 & 35.04 & 27.59 & 00.728 & 1485.5 \\
\hline & 085 & 00601 & 06.12 & 35.04 & 27.59 & & 1485.5 \\
\hline & 005 & 00653 & 05.58 & 35.02 & 27.64 & & 1484.2 \\
\hline & STO & 00700 & 05.21 & 35.01 & 27.68 & 00.783 & 1483.4 \\
\hline & 085 & 00702 & 05.20 & 35.01 & 27.68 & & 1483.4 \\
\hline & 085 & 00752 & 04.94 & 35.00 & 27.70 & & $14 d 3.2$ \\
\hline & STO & $\infty 0000$ & 04.76 & 35.01 & 27.73 & 00.832 & 1483.3 \\
\hline & 085 & 00801 & 04.76 & 35.01 & 27.73 & & 1483.3 \\
\hline & 085 & 00850 & 04.65 & 34.99 & 27.73 & & 1483.6 \\
\hline & 510 & 00900 & 04.57 & 34.99 & 27.74 & 00.879 & 1484.1 \\
\hline & 085 & 00902 & 04.56 & 34.99 & 27.74 & & 1484.1 \\
\hline & 085 & 00953 & 04.40 & 34.98 & 27.75 & & 1484.3 \\
\hline & 510 & 01000 & 04.31 & 34.97 & 27.75 & 00.925 & 1484.7 \\
\hline & 065 & 01001 & 04.31 & 34.97 & 27.75 & & 1484.7 \\
\hline & 085 & 01020 & 04.30 & 34.96 & 27.74 & & 1484.9 \\
\hline & 085 & 01082 & 04.22 & 34.97 & 27.76 & & 1485.7 \\
\hline & 085 & 01080 & 04.22 & 34.97 & 27.76 & & 1485.7 \\
\hline
\end{tabular}

$\ldots \ldots \ldots \ldots \ldots \ldots * \bullet$ 


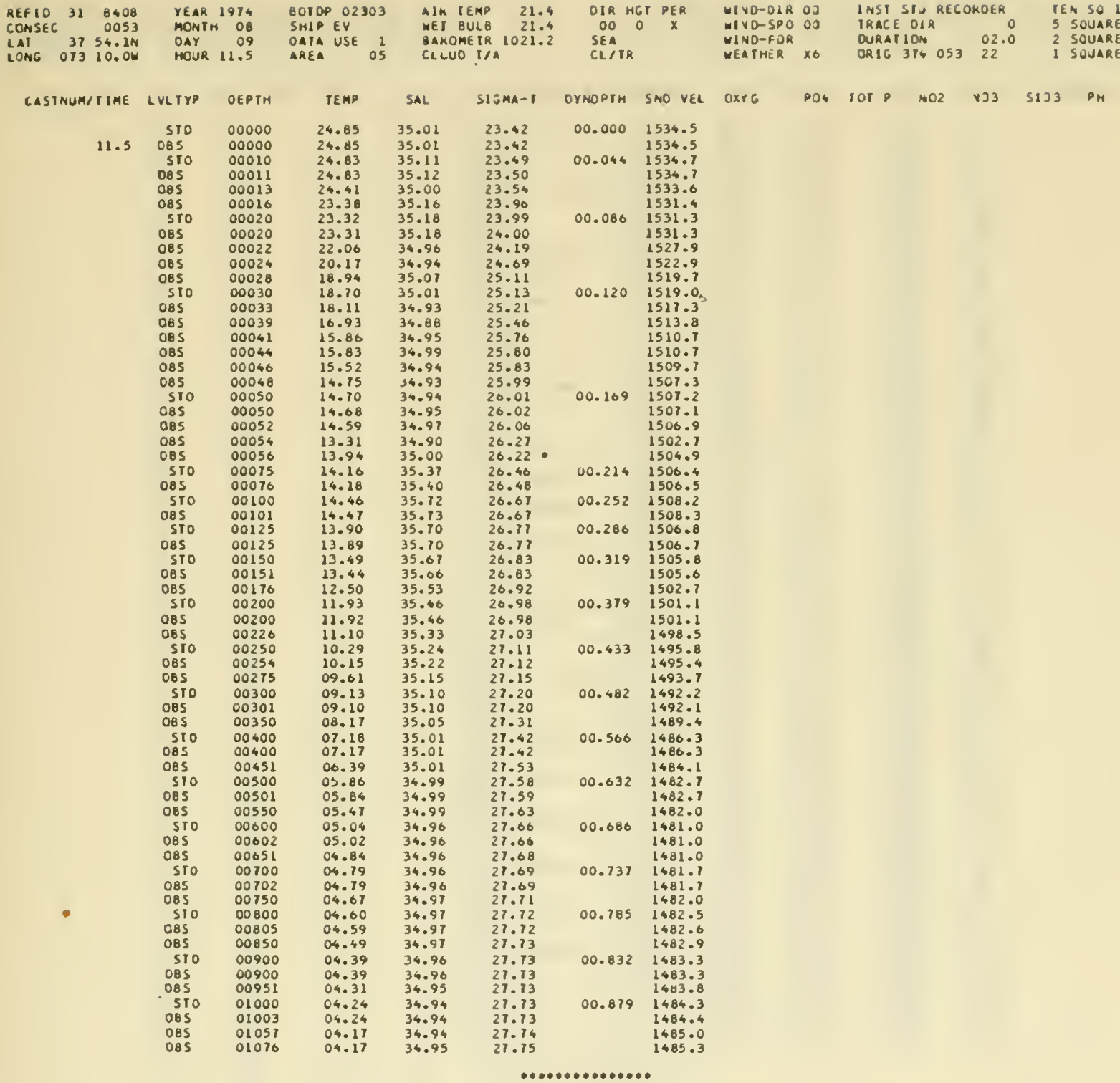




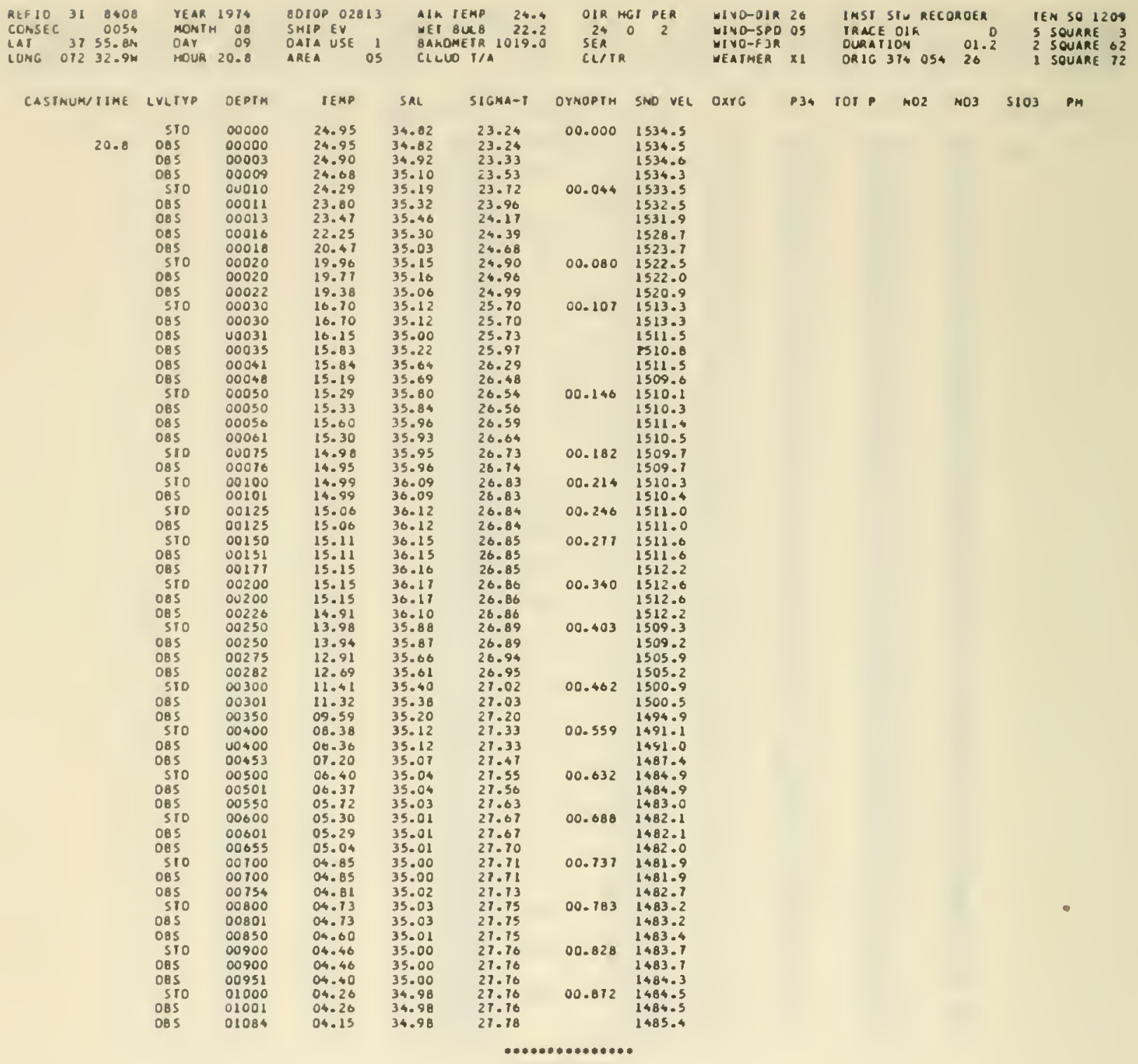




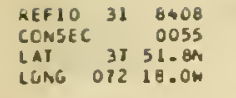
MONTH OB HOUR 23.8
BCTOP 03109 SHIP EV AKEA USE D
AIK IEMP 24.5 MEJ BUL 8 24.5 MEJ OUL8 24.5
OAROMETK 1017.2 CLLUO I/A

$\begin{array}{rrr}\text { OIR } & \text { HGI } & \text { PER } \\ \text { OO } & 0 & X \\ \text { SEA } & & \end{array}$
SEA
MIVDTOIA II MIVO-5PO 15 MINO-FJR WEAIHER XS
INSI STO RECONOER

IEN 521209 $\begin{array}{lll}\text { IRACE OIR } & 0 & 5 \text { SUUARE } 3 \\ \text { OURAIIOA } & \text { OL.1 } & 2 \text { SOUARE } 62\end{array}$ ORIG 374055 I SOJARE 72

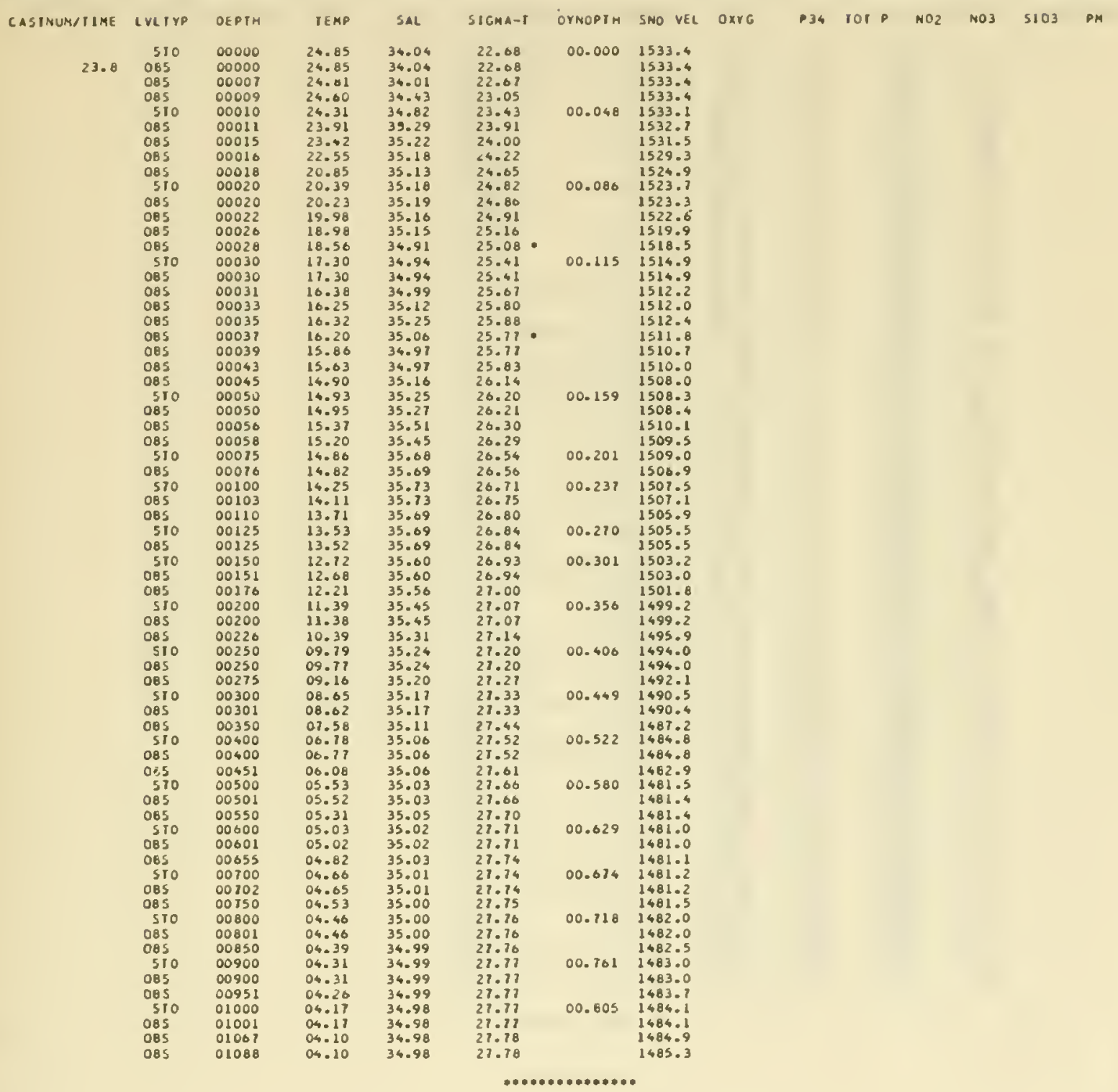




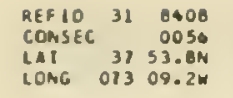

YEAR 19I4 $\begin{array}{ll}\text { MONIN } & 0 B \\ \text { OAY } & 12\end{array}$ MOUR 00.2
BOTDe OLAT

SHIP EV

OREA USE I itr iemp 21.9 WE I BULB 17.1 BA HOMEIR 1022.B
CLUUO I/A

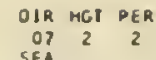
SE
MIVD-0IR 01 MIVD-SPO 16 WI VO-FOR WEATHER $X$
INSI SIN RECOROER OURAIION 01.8 S SOUARE 3 ORIG 370050 I SOUARE 73

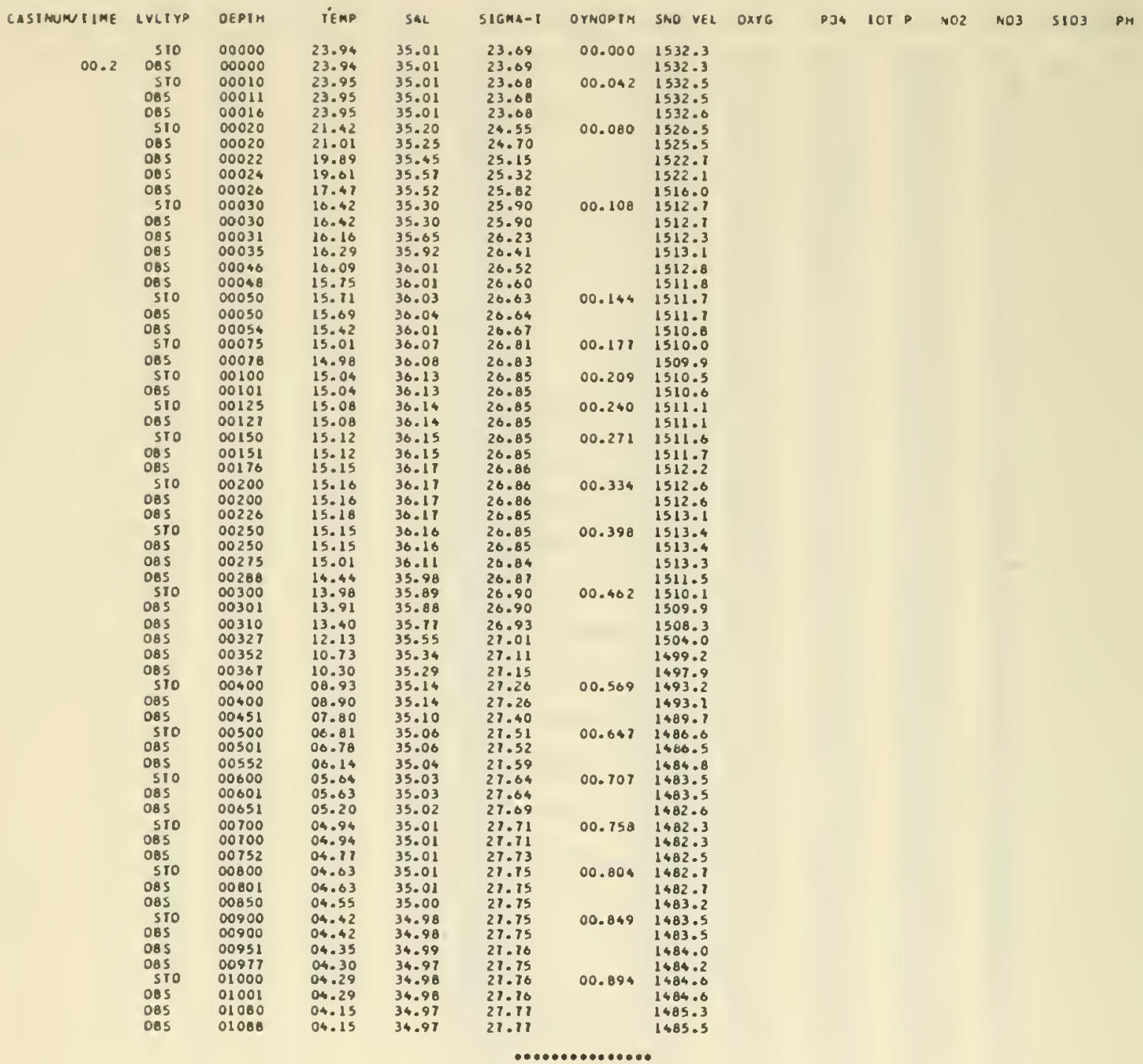




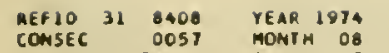
CONSEC OOST MONTH 08 $\begin{array}{lrrll} & 37 & 58.4 N & \text { OAY } & 12 \\ \text { LCNG } 073 & 14.8 Y & \text { HOUR } & 08.2\end{array}$

CASTNUMATIME LVLTYP OEPTH

\begin{tabular}{|c|c|c|}
\hline \multirow[t]{2}{*}{06.2} & $\begin{array}{l}510 \\
085\end{array}$ & $\begin{array}{l}00000 \\
00000\end{array}$ \\
\hline & 085 & 00005 \\
\hline & 570 & 00010 \\
\hline & 085 & 00010 \\
\hline & 085 & 00016 \\
\hline & 085 & 00019 \\
\hline & STO & 00020 \\
\hline & 085 & 00024 \\
\hline & 005 & \\
\hline & Sto & 00030 \\
\hline & 085 & 00034 \\
\hline & 085 & 00039 \\
\hline & 085 & 00044 \\
\hline & oes & 00049 \\
\hline & STO & 00050 \\
\hline & 085 & 00068 \\
\hline & 085 & 00073 \\
\hline & STO & 00075 \\
\hline & 085 & 00088 \\
\hline & OBS & 00098 \\
\hline & 5 To & 00100 \\
\hline & Des & 00123 \\
\hline & SIO & 00125 \\
\hline & 570 & 00150 \\
\hline & oes & 00197 \\
\hline & STO & 00200 \\
\hline & 085 & 00226 \\
\hline & 085 & 00246 \\
\hline & 510 & 00250 \\
\hline & 085 & 00295 \\
\hline & STO & 00300 \\
\hline & 005 & 00393 \\
\hline & 510 & 00400 \\
\hline & 0.85 & 00492 \\
\hline & S10 & 00500 \\
\hline & oos & 00590 \\
\hline & STo & 00600 \\
\hline & 085 & 00688 \\
\hline & STO & 00700 \\
\hline & Oes & 00787 \\
\hline & STO & 00800 \\
\hline & 005 & 00884 \\
\hline & 510 & 00900 \\
\hline & 085 & 00984 \\
\hline & 570 & 01000 \\
\hline & O8s & 06082 \\
\hline
\end{tabular}

OCTOP 02230

IN TEMP 23. OATA USE I BAROMETR 1021.9 AREA OS

BAROMETR 1021.9
CLLUD T/A

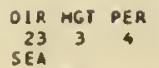

SEA

CL/TR
MINDTOIR 32

MIVO-SPO

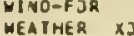

INST STO RECOROER

IRACE OIR

ORIG 376 OS
TEN 521209

5 SCURRE 35

2 SOUARE
1 SOJARE 73

$\begin{array}{lllll}\text { TEMP } & \text { SAL } & \text { S1GMA-T } & \text { OYNOPIH } & \text { SNO VEL } \\ & & & & \\ 23.87 & 35.11 & 23.78 & 00.000 & 1532.2 \\ 23.87 & 35.11 & 23.78 & & 1532.2 \\ 23.85 & 35.11 & 23.79 & & 1532.3 \\ 23.87 & 35.11 & 23.78 & 00.041 & 1532.4 \\ 23.87 & 35.11 & 23.78 & & 1532.4 \\ 23.74 & 35.05 & 23.78 & & 1532.1 \\ 23.74 & 35.05 & 23.78 & & 1532.2 \\ 23.47 & 35.07 & 23.87 & 00.082 & 1531.6 \\ 19.63 & 35.29 & 25.10 & & 1521.9 \\ 16.10 & 35.35 & 26.01 & & 1511.7 \\ 16.10 & 35.35 & 26.01 & 00.113 & 1511.7 \\ 16.10 & 35.35 & 26.01 & & 1511.8 \\ 15.38 & 35.53 & 26.31 & & 1509.9 \\ 15.58 & 35.77 & 26.45 & & 1510.9 \\ 15.61 & 35.84 & 26.50 & & 1511.1 \\ 15.61 & 35.85 & 26.51 & 00.148 & 1511.2 \\ 15.24 & 36.06 & 26.75 & & 1510.8 \\ 15.06 & 36.07 & 26.80 & & 1510.1 \\ 15.05 & 36.07 & 26.81 & 00.183 & 1510.1 \\ 14.99 & 36.10 & 26.84 & & 1510.2 \\ 15.04 & 36.10 & 26.83 & & 1510.5 \\ 15.04 & 36.10 & 26.83 & 00.215 & 1510.5 \\ 15.06 & 36.14 & 26.85 & & 1511.0 \\ 15.06 & 36.14 & 26.85 & 00.246 & 1511.0 \\ 15.12 & 36.16 & 26.85 & 00.278 & 1511.6 \\ 15.16 & 36.17 & 26.86 & & 1512.6 \\ 15.16 & 36.17 & 26.86 & 00.341 & 1512.6 \\ 15.14 & 36.17 & 26.86 & & 1513.0 \\ 14.84 & 36.09 & 26.86 & & 1512.3 \\ 14.71 & 36.06 & 26.87 & 00.404 & 1511.9 \\ 13.00 & 35.69 & 26.94 & & 1506.6 \\ 12.76 & 35.65 & 26.96 & 00.465 & 1505.8 \\ 09.00 & 35.14 & 27.25 & & 1493.3 \\ 08.82 & 35.13 & 27.27 & 00.569 & 1492.8 \\ 06.75 & 35.05 & 27.51 & & 1486.2 \\ 06.65 & 35.05 & 27.53 & 00.646 & 1486.0 \\ 05.70 & 35.05 & 27.65 & & 1483.7 \\ 05.61 & 35.05 & 27.66 & 00.704 & 1483.5 \\ 04.97 & 35.06 & 27.73 & & 1482.3 \\ 04.93 & 35.04 & 27.73 & 00.753 & 1482.3 \\ 04.66 & 35.01 & 27.74 & & 1482.6 \\ 04.83 & 35.01 & 27.75 & 00.798 & 1482.7 \\ 04.44 & 35.00 & 27.76 & & 1483.3 \\ 04.41 & 35.00 & 27.76 & 00.843 & 1483.5 \\ 146.28 & 35.00 & 27.78 & & 1484.3 \\ 04.26 & 35.00 & 27.78 & 00.886 & 1484.5 \\ 04.16 & 34.98 & 27.77 & & 1485.4\end{array}$

OXYG P34 TOTP VO2 NO3 SIO3 PH 


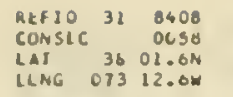

YLAR 1974 MONTH OB $\begin{array}{lr}\text { OAY } & 12 \\ \text { MOUR } 12.5\end{array}$
BOTOP 02280 SHIP EV
OAIA USE ARE A OS $\begin{array}{lll}\text { AIR TEMP } & 22.8 \\ \text { UL I BULB } & 19.1\end{array}$

- I BULB 19.1 OUO-JIR 32

OAMONETR 1021.0 SEA WINO-SPO IO

CLLUO I/A CLIIA

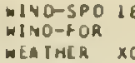

INSI SIJ RECORUER

IEN SO 1209

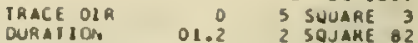

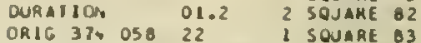

\begin{tabular}{|c|c|c|c|c|c|c|c|}
\hline \multirow[t]{2}{*}{ CASIMUNIIAE } & LVLTYP & OEPIM & IEMP & SAL & $516 M A-1$ & UYNOPIH & SNO VEL \\
\hline & STo & 00000 & 23.82 & 35.15 & 23.83 & 00.000 & 1532.2 \\
\hline \multirow[t]{62}{*}{12.5} & oos & 00000 & 23.82 & 35.15 & $\angle 3.83$ & & 1532.2 \\
\hline & S10 & 00010 & 23.83 & 35.15 & 23.83 & 00.041 & 1532.4 \\
\hline & OBS & 00011 & 23.83 & 35.25 & 23.83 & & 1532.4 \\
\hline & 085 & 00013 & 23.48 & 35.11 & 23.90 & & 1531.5 \\
\hline & oss & 00015 & 20.64 & 35.20 & 24.76 & & 1524.3 \\
\hline & 085 & 00016 & 19.98 & 35.58 & 25.23 & & 1523.0 \\
\hline & oss & 00018 & 14.89 & 35.66 & 25.31 & & 1522.9 \\
\hline & SIO & 00020 & 19.84 & 35.50 & 25.25 . & 00.075 & 1522.7 \\
\hline & oes & 00020 & 19.65 & 35.55 & 25.29 & & 1522.2 \\
\hline & OBS & 00022 & 18.43 & 35.66 & 25.69 & & 1518.9 \\
\hline & oos & 00026 & 18.22 & 35.40 & 25.56 & & 1518.0 \\
\hline & OBS & 00026 & 17.31 & 35.42 & 25.70 & & 1515.4 \\
\hline & OBS & 00028 & 16.90 & 35.41 & 25.86 & & 1514.4 \\
\hline & S10 & 00030 & 16.39 & 35.39 & 25.98 & 00.099 & 1512.7 \\
\hline & OBS & 00031 & 15.97 & 35.38 & 20.06 & & 1511.4 \\
\hline & OBS & 00041 & 18.00 & 35.98 & 26.52 & & 1512.4 \\
\hline & OBS & 00043 & 15.76 & 35.95 & 26.55 & & 1511.6 \\
\hline & STO & 00030 & 15.83 & 36.05 & 26.61 & 00.134 & 1512.1 \\
\hline & OBS & 00050 & 15.83 & 36.06 & 20.62 & & 1512.2 \\
\hline & 510 & 00075 & 15.02 & 36.08 & 20.82 & 00.100 & 1510.0 \\
\hline & OBS & 00070 & 14.94 & 36.08 & 26.82 & & 1509.9 \\
\hline & STo & 00100 & 15.01 & 36.12 & 20.65 & 00.199 & 1510.4 \\
\hline & OBS & 00101 & 15.02 & 36.12 & 26.85 & & 1510.5 \\
\hline & Sto & 00125 & 15.05 & 36.14 & 26.86 & 00.230 & 1511.0 \\
\hline & OBS & 00125 & 15.05 & 36.14 & 26.80 & & 1511.0 \\
\hline & sio & 00150 & 15.10 & 36.16 & 26.80 & 00.261 & 1511.0 \\
\hline & oes & 00151 & 15.10 & 36.16 & 26.86 & & 1511.0 \\
\hline & OBS & 00177 & 15.15 & 36.17 & 20.86 & & 1512.2 \\
\hline & SIO & 00200 & 15.15 & 36.18 & 26.87 & 00.324 & 1512.6 \\
\hline & OBS & 00200 & 15.15 & 36.18 & 26.87 & & 1512.6 \\
\hline & OBS & 00226 & 15.16 & 36.18 & 20.80 & & 1513.1 \\
\hline & Sio & 00250 & is. is & 36.18 & 20.86 & 00.387 & 1513.4 \\
\hline & 065 & 00230 & 15.14 & 36.18 & 26.87 & & 1513.4 \\
\hline & 085 & 00277 & 14.49 & 36.00 & 26.07 & & 1511.5 \\
\hline & 085 & 00286 & 14.23 & 35.97 & 26.90 & & 1510.8 \\
\hline & $\$ 10$ & 00300 & 13.34 & 35.80 & 26.96 & 00.449 & 1507.9 \\
\hline & oes & 00301 & 13.23 & 35.78 & 26.97 & & 1507.6 \\
\hline & 085 & 00309 & 12.63 & 35.06 & 27.00 & & 1505.5 \\
\hline & OBS & 00322 & 11.95 & 35.55 & 27.04 & & 1503.3 \\
\hline & OBS & 00350 & 10.40 & 35.34 & 27.10 & & 1498.2 \\
\hline & 510 & 00400 & 08.83 & 35.16 & 27.29 & 00.552 & 1492.8 \\
\hline & 085 & 00400 & 08.80 & 35.16 & 27.30 & & 1492.7 \\
\hline & OBS & 00408 & 08.55 & 35.14 & 27.32 & & 1491.9 \\
\hline & 085 & 00453 & 07.56 & 35.08 & 27.42 & & 1488.8 \\
\hline & 510 & Ous00 & 00.62 & 35.07 & 27.52 & 00.028 & 1486.7 \\
\hline & Oes & 00305 & 08.74 & 35.07 & 27.53 & & 1486.6 \\
\hline & 085 & 00539 & 08.26 & 35.00 & 27.59 & & 1485.1 \\
\hline & S10 & 00600 & 05.79 & 35.04 & 27.03 & 00.688 & 1484.2 \\
\hline & 510 & 00700 & 05.03 & 35.03 & 27.12 & 00.739 & 1482.1 \\
\hline & oBs & 00705 & 04.98 & 35.03 & 27.12 & & 1482.6 \\
\hline & OBS & 00713 & 04.99 & 35.03 & 27.72 & & 1482.8 \\
\hline & 085 & 00750 & 04.60 & 35.02 & $27.7^{4}$ & & 1482.6 \\
\hline & STO & 00800 & 04.60 & 35.01 & 27.75 & 00.785 & 1482.6 \\
\hline & oes & 00801 & 04.60 & 35.01 & 27.75 & & 1682.6 \\
\hline & 085 & 00850 & 04.51 & 35.00 & 27.15 & & 1403.0 \\
\hline & 510 & 00900 & 04.60 & 35.00 & 27.70 & 00.829 & 1483.4 \\
\hline & OBS & 00900 & 04.40 & 35.00 & 27.76 & & 1483.4 \\
\hline & obs & 00951 & 04.30 & 34.99 & 27.77 & & 1403.8 \\
\hline & 510 & 02000 & 04.21 & 34.99 & 27.17 & 00.873 & 1484.5 \\
\hline & 085 & 01003 & 04.27 & 36.99 & 27.77 & & 1484.6 \\
\hline & 085 & 01007 & 04.19 & 34.98 & 27.77 & & 1485.3 \\
\hline & OBS & 01084 & 04.15 & 34.99 & 27.78 & & 1485.4 \\
\hline
\end{tabular}




$\begin{array}{llll}\text { REFIO } 31 & 8406 & \text { YEAR 1974 } \\ \text { CONSEC } & \text { OOS9 } & \text { MONTH OB } \\ \text { LAT } 36 & 10.2 \mathrm{~N} & \text { DAY } 13 \\ \text { LONG OT3 OO. } & \text { MOUR OS.3 }\end{array}$

BOTOP 02281

SMIP EY

OATA USE
IK IEMP 22.8
WET OULA OIR HGI PER
BAROAETR 1018.9
CLCUO TIA
WIVO-DIR 32

WIVO-SPO 15

WIVD-FJR

WEATHCR 10
IHST STD RECCROER

TRACE DIR

OURAIIOA

ORIG $37 \%$ OSO
TEM 501208 5 SQUARE ? 2 SOUARE 2 
REP10 $31 \quad 0400$ YEAR 1974 CONSEC 0060 MONIM OB

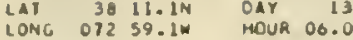

BOTOP 02455

SHIP EY

OATA USE I

ARE OS

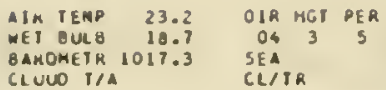

WVO-0IE 32
WIVO-SPO 18

IIVO-F $3 A$

WEAIMER $X$
INST STD RECOROER

TRACE OIR

OURATION
ORIC 376 OS
IEN SO 1209

5 SOJARE 3

2 SOUARE 22

\begin{tabular}{|c|c|c|c|c|c|c|c|}
\hline \multirow[t]{65}{*}{ CASTMUMIIINE } & LVLTYP & OEPTH & TEMP & SAL & $\operatorname{Sig} A 4-1$ & OYNOPTH & SNO VEL \\
\hline & STO & 00000 & 23.05 & 35.11 & 23.85 & 00.000 & 1531.7 \\
\hline & oes & 00000 & 23.05 & 35.11 & 23.85 & & 1531.1 \\
\hline & 510 & 00010 & 23.66 & 35.11 & 23.85 & 00.041 & 1531.9 \\
\hline & oss & 00013 & 23.66 & 35.11 & 23.85 & & 1531.9 \\
\hline & STO & 00020 & 23.67 & 35.12 & 23.85 & 00.001 & 1532.1 \\
\hline & oes & 00020 & 23.67 & 35.12 & 23.85 & & 1532.1 \\
\hline & OBS & 00022 & 23.37 & 35.02 & 23.86 & & 1531.3 \\
\hline & 005 & 00024 & 22.78 & 35.33 & 24.27 & & 1530.2 \\
\hline & OES & 00028 & 20.03 & 35.31 & 25.01 & & 1523.0 \\
\hline & STO & 00030 & 19.10 & 35.55 & 25.44 & 00.115 & 1520.8 \\
\hline & oos & 00031 & 10.19 & 35.65 & 25.74 & & 1518.3 \\
\hline & $\begin{array}{l}085 \\
085\end{array}$ & $\begin{array}{l}00035 \\
00037\end{array}$ & $\begin{array}{l}16.68 \\
15.91\end{array}$ & $\begin{array}{l}35.42 \\
35.53\end{array}$ & $\begin{array}{l}25.98 \\
26.19\end{array}$ & & $\begin{array}{l}1513.1 \\
1511.5\end{array}$ \\
\hline & 065 & 00041 & 15.67 & 35.78 & 26.46 & & 1511.1 \\
\hline & 085 & 00043 & 15.91 & 35.88 & 26.46 & & 1512.0 \\
\hline & OBS & 00045 & 15.93 & 35.87 & 26.45 & & 1512.1 \\
\hline & Sic & 00050 & 15.07 & 35.88 & 26.52 & 00.156 & 1511.4 \\
\hline & OBS & 00052 & 15.54 & 35.88 & 26.55 & & 1511.0 \\
\hline & 085 & 00054 & 15.69 & 36.03 & 26.63 & & 1511.7 \\
\hline & 085 & 00001 & 15.52 & 36.03 & 26.67 & & 1511.3 \\
\hline & STO & 00075 & 15.21 & 36.04 & 26.76 & 00.192 & 1510.6 \\
\hline & OBS & 00076 & 15.18 & 36.04 & 26.75 & & 1510.5 \\
\hline & sro & 00100 & 15.03 & 36.10 & 26.83 & 00.226 & 1510.5 \\
\hline & 085 & 00103 & 15.02 & 36.10 & 20.83 & & 1510.5 \\
\hline & STo & 00125 & 15.04 & 30.12 & 26.86 & 00.256 & 1510.9 \\
\hline & Oos & 00125 & 15.04 & 36.12 & 20.84 & & 1510.9 \\
\hline & 510 & 00150 & 15.09 & 36.15 & 26.86 & 00.287 & 1511.5 \\
\hline & OBS & 00151 & 15.09 & 36.15 & 26.86 & & 1511.6 \\
\hline & OBS & 00177 & 15.15 & 36.16 & 26.85 & & 1512.2 \\
\hline & STO & 00200 & 15.16 & 36.17 & 26.85 & 00.350 & 1512.6 \\
\hline & oss & 00204 & 15.16 & 36.17 & 26.86 & & 1512.7 \\
\hline & 085 & 00226 & 15.16 & 36.17 & 26.86 & & 1513.0 \\
\hline & STO & 00250 & is.17 & 30.17 & 20.85 & 00.414 & 1513.5 \\
\hline & 085 & 00252 & 15.17 & 30.17 & 26.85 & & 1513.5 \\
\hline & OBS & 00275 & 14.84 & 36.08 & 20.86 & & 1512.7 \\
\hline & Sio & 00300 & 13.81 & 35.80 & 20.80 & 00.478 & 1509.5 \\
\hline & $\begin{array}{l}\text { OBS } \\
\text { oos }\end{array}$ & $\begin{array}{l}00303 \\
00305\end{array}$ & $\begin{array}{l}13.34 \\
13.06\end{array}$ & $\begin{array}{l}35.76 \\
35.76\end{array}$ & $\begin{array}{l}26.93 \\
26.97\end{array}$ & & $\begin{array}{l}1507.9 \\
1507.0\end{array}$ \\
\hline & 085 & 00309 & 13.04 & 35.73 & 26.97 & & 1507.0 \\
\hline & OBS & 00312 & 12.55 & 35.64 & 27.00 & & 1505.3 \\
\hline & 085 & 00314 & 12.51 & 35.62 & 26.99 & & 1505.1 \\
\hline & oes & 00323 & 11.66 & 35.49 & 27.05 & & 1502.2 \\
\hline & $08 \mathrm{~S}$ & 00346 & 10.80 & 35.38 & 27.13 & & 1499.4 \\
\hline & OBS & 00350 & 10.47 & 35.30 & 27.12 & & 1498.2 \\
\hline & S10 & 00400 & 08.51 & 35.11 & 27.30 & 00.585 & 1691.6 \\
\hline & o8s & 00400 & 08.49 & 35.11 & 27.30 & & 1491.5 \\
\hline & obs & 00451 & 07.41 & 35.08 & 27.46 & & 1488.2 \\
\hline & 510 & 00500 & 06.47 & 35.06 & 27.56 & 00.658 & 1485.3 \\
\hline & 085 & 00501 & 06.44 & 35.06 & 27.56 & & 1685.2 \\
\hline & oos & 00550 & 05.81 & 35.04 & 27.63 & & 1483.4 \\
\hline & Sio & 00000 & 05.20 & 35.02 & 27.68 & 00.114 & 1482.0 \\
\hline & $\begin{array}{l}085 \\
085\end{array}$ & $\begin{array}{l}00601 \\
00051\end{array}$ & $\begin{array}{l}05.25 \\
05.06\end{array}$ & $\begin{array}{l}35.02 \\
35.03\end{array}$ & $\begin{array}{l}27.68 \\
27.12\end{array}$ & & $\begin{array}{l}1482.0 \\
1482.0\end{array}$ \\
\hline & sio & 00700 & 04.91 & 35.03 & 27.73 & 00.762 & 1482.2 \\
\hline & 005 & 00700 & 04.91 & 35.03 & 27.73 & & 1482.2 \\
\hline & obs & 00750 & 04.76 & 35.01 & 27.73 & & 1482.4 \\
\hline & SIO & 00800 & 04.60 & 35.01 & 27.75 & 00.807 & 1482.6 \\
\hline & oes & 00801 & 04.60 & 35.01 & 27.75 & & $1682.6^{\circ}$ \\
\hline & oes & 00850 & 04.51 & 35.00 & 27.75 & & 1483.0 \\
\hline & STo & 40900 & 04.39 & 34.99 & 27.76 & 00.851 & 1483.4 \\
\hline & 085 & 00902 & 04.39 & 34.99 & 27.76 & & 1483.4 \\
\hline & oes & 00951 & 04.31 & 34.99 & 27.77 & & 1483.9 \\
\hline & S10 & 01000 & 04.26 & 36.98 & 27.76 & 00.896 & 1484.5 \\
\hline & OES & 01001 & 04.26 & 34.98 & 27.76 & & 1604.5 \\
\hline & 085 & 01056 & 04.14 & 34.96 & 27.76 & & 1484.9 \\
\hline & 085 & 01078 & 04.12 & 34.97 & 27.77 & & 1485.2 \\
\hline & 085 & 01082 & 04.12 & 34.91 & 27.77 & & 1485.2 \\
\hline
\end{tabular}




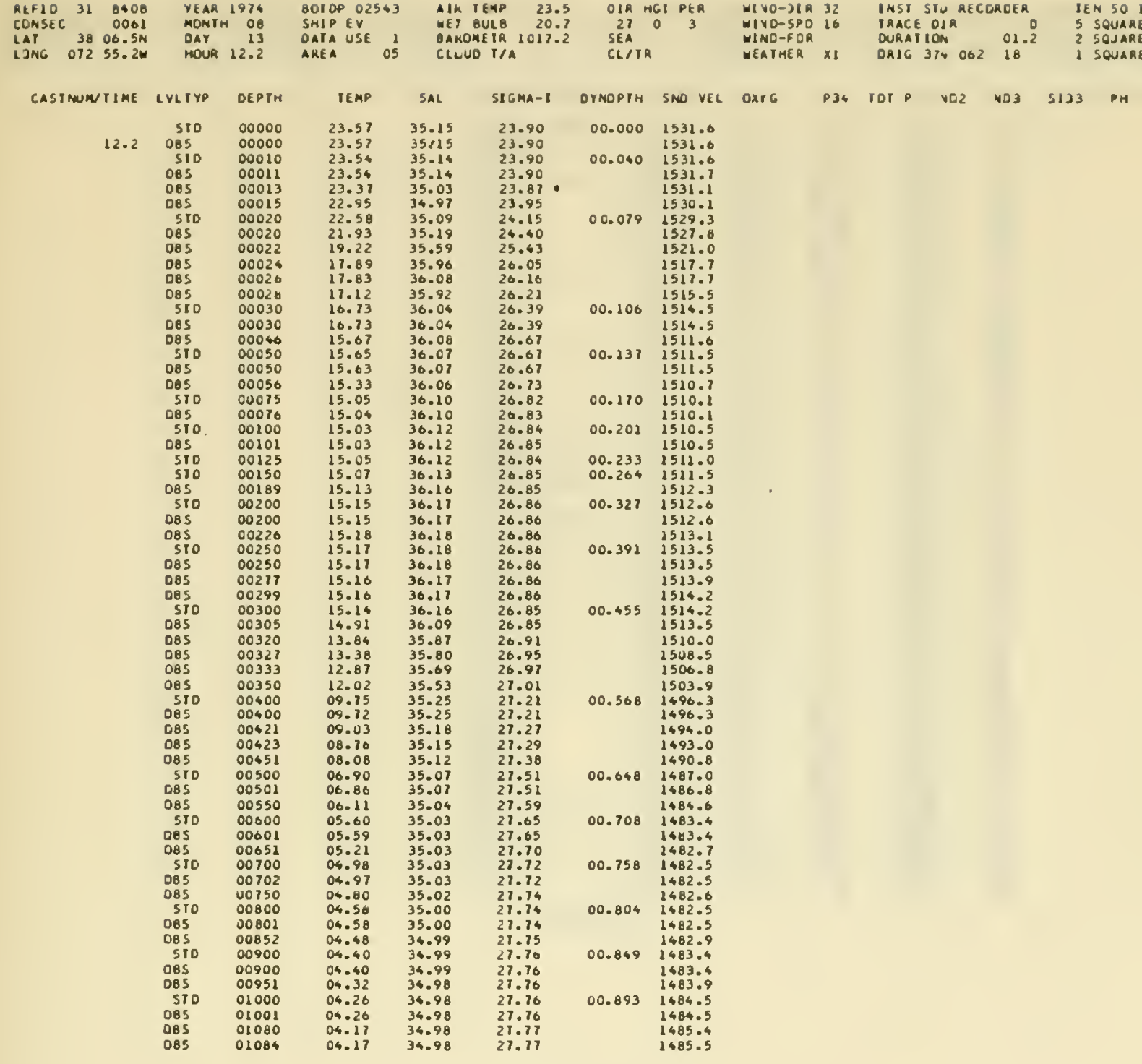




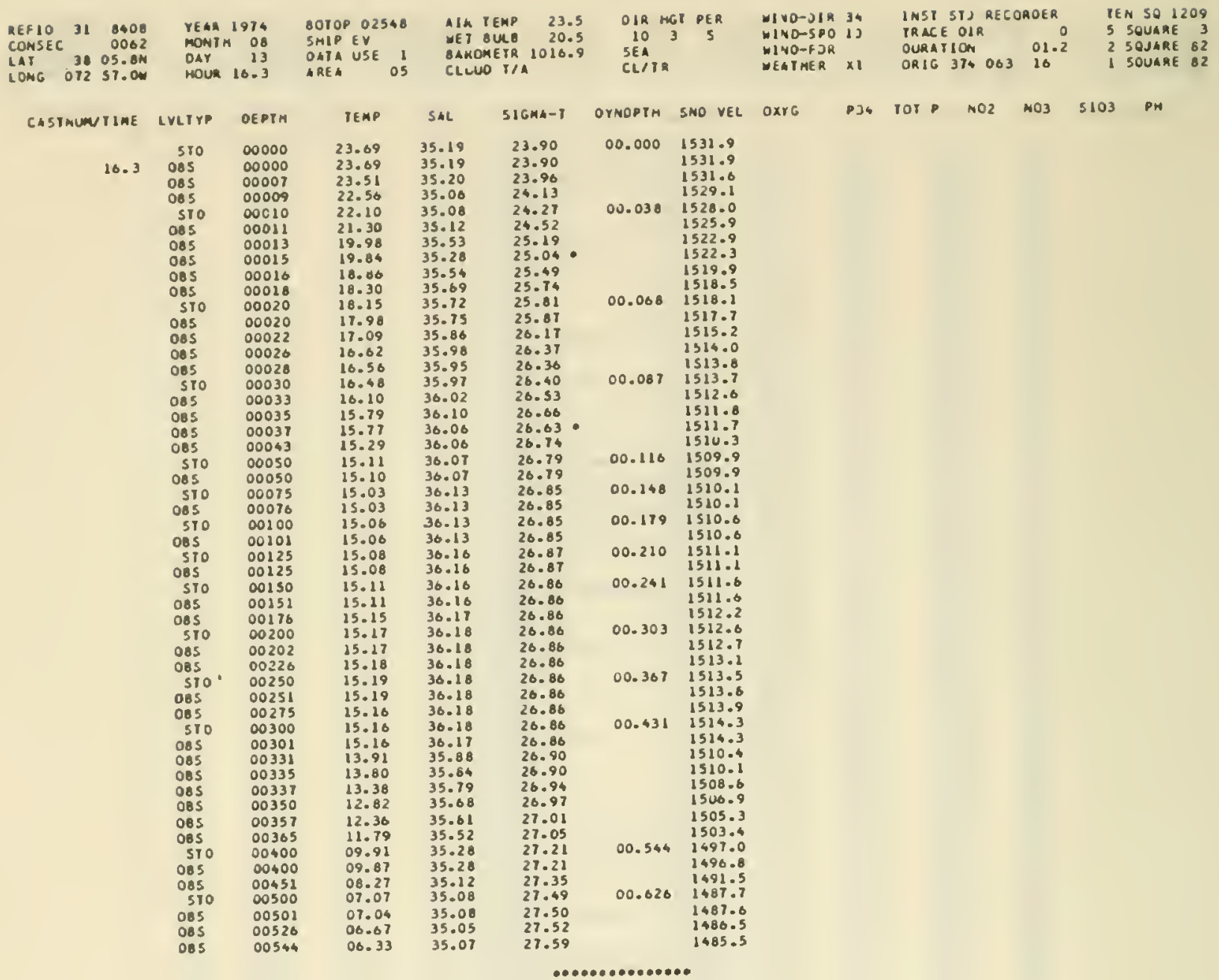


REF10 310408 YEAR 1974

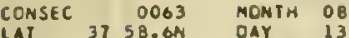
LONG $07242.3 \mathrm{~W}$ MOUR 19.0
6010P 02707

SHIP EV

OATA USE I
AREA

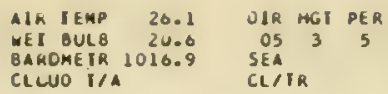

WIVD-OIR 27

$M I V O-2 P 0 \quad 10$

MINOEFTR
IN5T SIN RCCOROLA IEN SO 1209 TRACE OAR O 5 SUUARE $\begin{array}{lll}\text { DURATION } & 00.4 & 2 \text { SOUARE } 62 \\ \text { ORIG } 37 \% \text { OS4 } 19 & 1 \text { SOUARE } 72\end{array}$

\begin{tabular}{|c|c|c|c|c|c|c|c|}
\hline \multirow{52}{*}{$\begin{array}{r}\text { CASTNUM/I IKE } \\
18.0\end{array}$} & LVLTYP & OEPIH & IEAP & Sil & $S I S M A-T$ & OYNOPTH & SND VEL \\
\hline & 570 & 00000 & 23.31 & 35.62 & 24.33 & 00.000 & 1531.4 \\
\hline & oos & 00000 & 23.31 & 35.62 & 26.33 & & 1531.4 \\
\hline & STO & 00010 & 22.95 & 35.60 & 24.42 & 00.036 & 1530.7 \\
\hline & 085 & 00011 & 22.93 & 35.60 & 24.43 & & 1530.1 \\
\hline & oss & 00013 & 22.90 & 35.60 & 24.44 & & 1530.6 \\
\hline & oos & 00015 & 21.95 & 35.50 & 24.63 & & 1528.1 \\
\hline & OBS & 00018 & 20.82 & 35.50 & 24.94 & & 1525.2 \\
\hline & STO & 00020 & 18.39 & 35.50 & 25.57 & 00.005 & 1518.6 \\
\hline & 085 & 00020 & 17.09 & 35.50 & 25.75 & & 1510.5 \\
\hline & 085 & 00022 & 17.15 & 35.67 & 26.01 & & 1515.2 \\
\hline & 085 & 00028 & 17.08 & 36.01 & 26.29 & & 1513.5 \\
\hline & STO & 00030 & 16.97 & 36.00 & 26.31 & 00.060 & 1515.2 \\
\hline & OAS & 00033 & 16.49 & 35.99 & 26.41 & & 1513.8 \\
\hline & 085 & 00035 & 16.11 & 35.99 & 26.50 & & 1512.6 \\
\hline & OBS & 00039 & 15.85 & 30.05 & 26.61 & & 1512.0 \\
\hline & \$10 & 00050 & 15.21 & 36.06 & 20.76 & 00.117 & 1510.2 \\
\hline & OBS & 00050 & 15.18 & 36.06 & 26.77 & & 1510.1 \\
\hline & 510 & 00075 & 15.03 & 36.14 & 26.86 & 00.148 & 1510.1 \\
\hline & OBS & 00076 & 15.03 & 36.14 & 26.86 & & 1510.1 \\
\hline & STO & 00100 & 15.06 & 30.14 & 20.85 & 00.179 & 1510.0 \\
\hline & OBS & 00101 & 15.06 & 30.14 & 20.85 & & 1510.6 \\
\hline & 510 & 00125 & 15.08 & 36.15 & 26.80 & 00.210 & 1511.1 \\
\hline & OBS & 00125 & 15.08 & 36.15 & 26.86 & & 1511.1 \\
\hline & STo & 00150 & 15.13 & 36.16 & 26.85 & 00.241 & 1511.7 \\
\hline & OBS & 00151 & 15.13 & 36.16 & 26.85 & & 1511.7 \\
\hline & DBS & 00170 & 15.15 & 36.17 & 26.86 & & 1512.2 \\
\hline & STO & 00208 & 15.17 & 36.19 & 20.87 & 00.304 & 1512.7 \\
\hline & 085 & 00200 & 15.17 & 36.19 & 20.87 & & $151<.7$ \\
\hline & 085 & 00243 & 15.15 & 36.19 & 20.87 & & 1513.3 \\
\hline & STO & 00250 & 15.14 & 36.17 & 26.86 & 00.367 & 1513.4 \\
\hline & 085 & 00251 & 15.16 & 36.17 & 20.86 & & 1513.4 \\
\hline & 085 & 00277 & 15.26 & 30.17 & 26.86 & & 1513.9 \\
\hline & STO & 00300 & 14.79 & 36.08 & 20.80 & 00.431 & 1513.0 \\
\hline & OBS & 00301 & 14.75 & 36.07 & 26.87 & & 1512.9 \\
\hline & 085 & 00310 & 14.46 & 36.04 & 26.91 & & 1512.0 \\
\hline & 085 & 00316 & 14.08 & 35.94 & 20.91 & & 1510.8 \\
\hline & OBS & 00318 & 13.99 & 35.91 & 20.91 & & 1510.5 \\
\hline & OQS & 00325 & 13.38 & 35.78 & 20.94 & & 1508.5 \\
\hline & 085 & 00329 & 13.24 & 35.77 & 20.90 & & 1508.0 \\
\hline & 085 & 00333 & 12.92 & 35.71 & 20.98 & & 1507.0 \\
\hline & OBS & 00350 & 12.02 & 35.57 & 27.05 & & 1506.0 \\
\hline & 085 & 00355 & 11.97 & 35.57 & 27.06 & & 1503.9 \\
\hline & 085 & 00361 & 11.55 & 35.47 & 27.06 & & 1502.4 \\
\hline & 510 & 00400 & 09.88 & 35.20 & 27.20 & 00.544 & 1490.9 \\
\hline & OBS & $0 \cup 400$ & 09.87 & 35.26 & 27.20 & & 1490.8 \\
\hline & 085 & 00404 & 09.82 & 35.28 & 27.22 & & 1490.7 \\
\hline & 085 & 00451 & 08.33 & 35.13 & 27.35 & & 1491.8 \\
\hline & 570 & 00500 & 07.54 & 35.08 & 27.42 & 00.630 & 1489.5 \\
\hline & DBS & 00500 & 07.54 & 35.08 & 27.42 & & 1489.5 \\
\hline & 085 & 00533 & 06.76 & 35.04 & 27.50 & & 1486.9 \\
\hline & OBS & 00548 & 00.49 & 35.08 & 27.57 & & 1400.2 \\
\hline
\end{tabular}




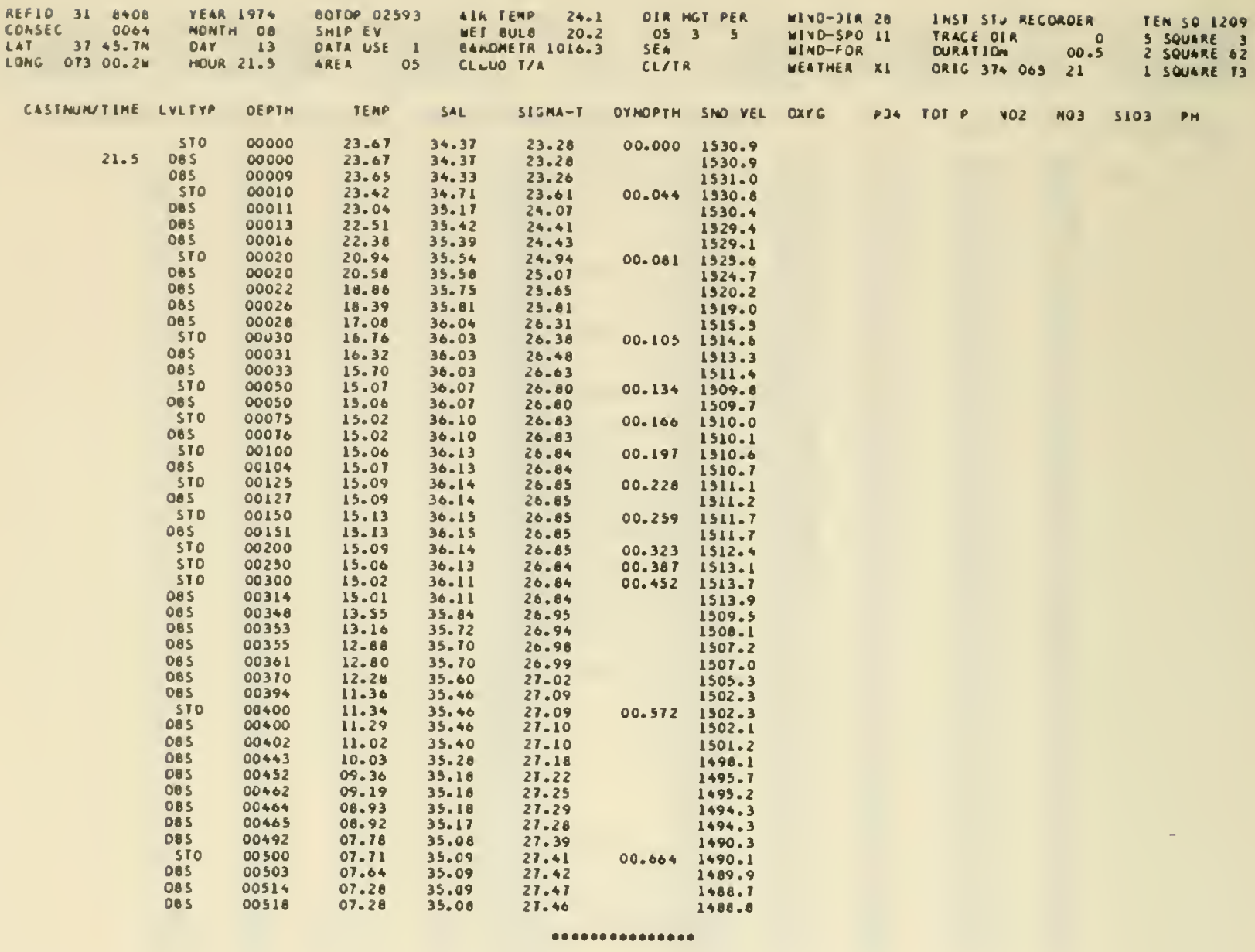




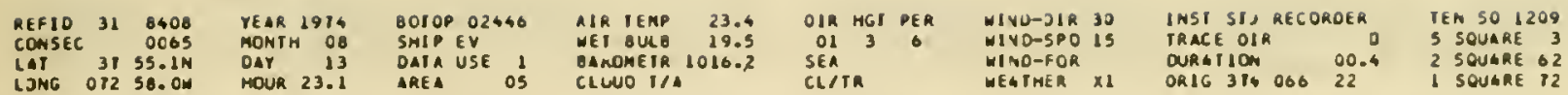

\begin{tabular}{|c|c|c|c|c|c|c|c|}
\hline \multirow[t]{2}{*}{ CASTMUN TIME } & LVLTVP & OEPIN & TENP & SAL & $S I G M A-T$ & DYMOPIN & SNO VEL \\
\hline & STO & 00000 & 23.50 & 34.36 & 23.33 & 00.000 & 1530.5 \\
\hline \multirow[t]{44}{*}{23.1} & 085 & 00000 & 23.50 & 34.36 & 23.33 & & 1530.5 \\
\hline & 005 & 00005 & 23.49 & 34.39 & 23.35 & & 1530.6 \\
\hline & 085 & 00007 & 22.95 & 34.99 & 23.96 & & 1530.0 \\
\hline & DOS & 00009 & 22.19 & 35.52 & 24.58 & & 1528.7 \\
\hline & & 00010 & 21.69 & 35.57 & 26.75 & 00.039 & 1527.4 \\
\hline & oes & 00013 & 19.59 & 35.15 & 25.46 & & 1522.1 \\
\hline & OQS & 00016 & 18.15 & 35.66 & 25.91 & & 1518.2 \\
\hline & & $\begin{array}{l}00018 \\
00020\end{array}$ & 17.39 & 35.93 & 26.15 & & 1516.1 \\
\hline & 510 & $\begin{array}{l}00020 \\
00020\end{array}$ & $\begin{array}{l}17.30 \\
17.22\end{array}$ & $\begin{array}{l}35.96 \\
35.98\end{array}$ & 26.20 & 00.064 & $\begin{array}{l}1515.9 \\
1515.7\end{array}$ \\
\hline & $\begin{array}{l}085 \\
085\end{array}$ & $\begin{array}{l}00020 \\
00022\end{array}$ & $\begin{array}{l}17.22 \\
16.76\end{array}$ & $\begin{array}{l}35.98 \\
36.05\end{array}$ & $\begin{array}{l}26.23 \\
26.39\end{array}$ & & $\begin{array}{l}1515.7 \\
1516.5\end{array}$ \\
\hline & $\begin{array}{l}085 \\
085\end{array}$ & 00028 & 16.63 & 36.02 & 26.40 & & 1514.1 \\
\hline & STO & 00030 & 15.94 & 36.06 & 26.56 & 00.081 & 1512.1 \\
\hline & Dos & 00030 & 15.94 & 36.04 & 26.58 & & 1512.1 \\
\hline & STO & 00050 & 15.09 & 36.07 & 26.79 & 00.108 & $\begin{array}{l}1509.8 \\
1509.8\end{array}$ \\
\hline & Oos & 00050 & 15.08 & 36.07 & 26.80 & & $\begin{array}{l}1509.8 \\
1510.1\end{array}$ \\
\hline & $\begin{array}{l}510 \\
0 \text { OS }\end{array}$ & $\begin{array}{l}00075 \\
00076\end{array}$ & $\begin{array}{l}15.04 \\
15.04\end{array}$ & $\begin{array}{l}36.10 \\
36.10\end{array}$ & $\begin{array}{l}26.83 \\
26.83\end{array}$ & 00.140 & $\begin{array}{l}1510.1 \\
1510.1\end{array}$ \\
\hline & STO & 00100 & 15.02 & 36.11 & 26.84 & 00.171 & 1510.5 \\
\hline & 085 & 00101 & 15.02 & 36.11 & 26.04 & & 1510.5 \\
\hline & $5 T 0$ & 00125 & 15.06 & 36.13 & 26.85 & 00.202 & 2511.0 \\
\hline & OBS & 00125 & 15.06 & 36.13 & 26.85 & & 1511.0 \\
\hline & $\begin{array}{l}\text { STO } \\
\text { OBS }\end{array}$ & $\begin{array}{l}00150 \\
00151\end{array}$ & $\begin{array}{l}15.09 \\
15.09\end{array}$ & $\begin{array}{l}36.14 \\
36.14\end{array}$ & $\begin{array}{l}26.85 \\
26.85\end{array}$ & 00.234 & $\begin{array}{l}1511.5 \\
1511.6\end{array}$ \\
\hline & 085 & 00176 & 25.15 & 36.15 & 26.84 & & 1512.2 \\
\hline & STO & 00200 & 15.18 & 36.17 & 26.05 & 00.297 & 1512.7 \\
\hline & OAS & 00200 & 15.18 & 36.17 & 26.85 & & 1512.7 \\
\hline & 085 & 00226 & 15.18 & 36.17 & 26.85 & & 1513.1 \\
\hline & STO & 00250 & 15.21 & 36.17 & 26.84 & 00.361 & $\begin{array}{l}1513.6 \\
1513.6\end{array}$ \\
\hline & $\begin{array}{l}085 \\
085\end{array}$ & 00275 & $\begin{array}{l}15.21 \\
15.16\end{array}$ & $\begin{array}{l}36.17 \\
36.16\end{array}$ & $\begin{array}{l}26.84 \\
26.85\end{array}$ & & $\begin{array}{l}1513.6 \\
1513.8\end{array}$ \\
\hline & 510 & 00300 & 15.16 & 36.16 & 26.85 & 00.426 & 1514.2 \\
\hline & Oes & 00301 & 15.16 & 36.16 & 20.85 & & 1514.3 \\
\hline & 085 & 00352 & 15.17 & 36.16 & 26.85 & & $\begin{array}{l}1513.1 \\
1513.3\end{array}$ \\
\hline & 085 & 00361 & 14.61 & 35.99 & 26.84 & & $\begin{array}{l}1513.3 \\
1513.0\end{array}$ \\
\hline & 085 & 00363 & 14.51 & 36.00 & 26.87 & & $\begin{array}{l}1513.0 \\
1510.4\end{array}$ \\
\hline & 085 & 00374 & 13.71 & 35.86 & 26.93 & & 1510.4 \\
\hline & 085 & 00360 & 13.19 & 35.12 & 26.93 & & 1508.6 \\
\hline & 085 & 00382 & 13.01 & 35.70 & 26.95 & & 1508.0 \\
\hline & STo & 00400 & 12.09 & 35.57 & 27.04 & 00.548 & 1505.0 \\
\hline & 085 & 00401 & 12.00 & 35.56 & 27.04 & & 1504.8 \\
\hline & 085 & 00410 & 11.24 & 35.42 & 21.08 & & $\begin{array}{l}1502.1 \\
1499.7\end{array}$ \\
\hline & 085 & 00423 & 10.54 & 35.36 & 21.16 & & 1499.7 \\
\hline & 085 & 00425 & 10.56 & 35.37 & 21.17 & & 1499.8 \\
\hline & 085 & 00451 & 09.46 & 35.21 & 27.23 & & 1496.0 \\
\hline & STO & 00500 & 07.84 & 35.08 & 27.38 & 00.645 & $\begin{array}{l}1490.6 \\
1490.6\end{array}$ \\
\hline & 085 & 00500 & 07.83 & 35.08 & 27.38 & & 1490.6 \\
\hline & 085 & $00 \$ 19$ & 07.44 & 35.06 & 27.42 & & 1489. \\
\hline
\end{tabular}




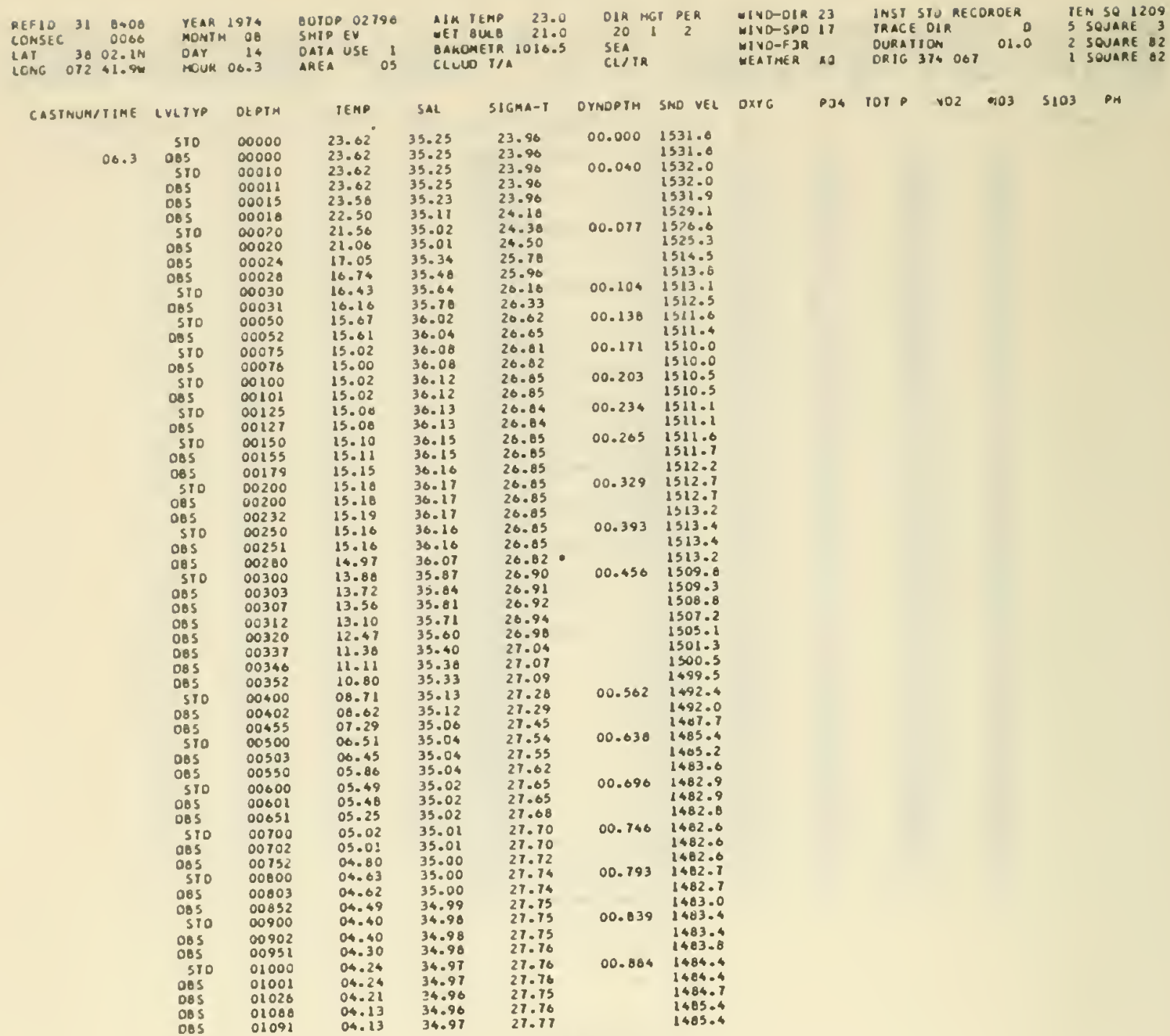




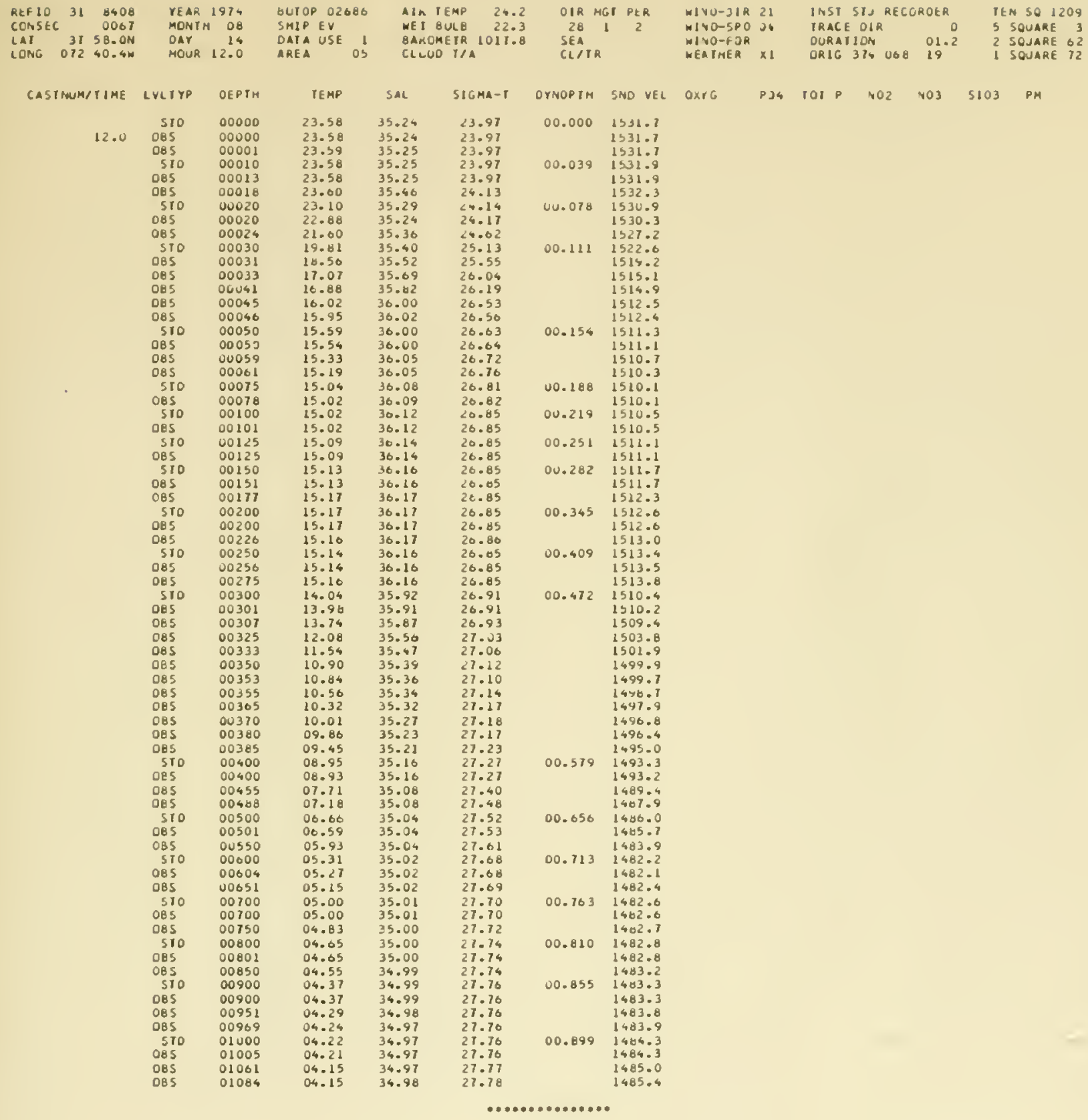






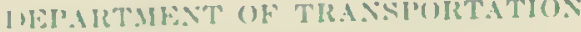

UNITED STATES COAST GUAKD

Washington, I).C. 2(15ik)

(Dtticla! Ibusiness

PEYALTY FOR PRIVATE, USE, 300
Hostagl: aND Frins PAIU L NITED STATES rOAST GUARI)

DOT 514 